\title{
The Role of PSD-95 and Kinase Interactions in Synaptic Transmission
}

\author{
$\mathrm{PhD}$ Thesis \\ in partial fulfilment of the requirements \\ for the degree \\ Doctor rerum naturalium (Dr. rer. nat.) \\ in the Neuroscience Program \\ at the Georg-August University Göttingen, \\ Faculty of Biology \\ submitted by \\ Seniye Derya Akad \\ born in \\ BadEms, Germany
}

Göttingen, March 2013 



\section{Thesis Committee Members:}

Dr. Dr. Oliver M. Schlüter (Reviewer)

Molecular Neurobiology, European Neuroscience Institute, Göttingen

Prof. Dr. Nils Brose (Reviewer)

Molecular Neurobiology, Max Planck Institute Experimental Medicine, Göttingen

Dr. Till Marquardt

Developmental Neurobiology, European Neuroscience Institute, Göttingen

\section{Extended Thesis Committee Members:}

Prof. Dr. Michael Hörner

Cellular Neurobiology, Johann-Friedrich-Blumenbach-Institute for Zoology and Anthropology, Göttingen

Prof. Dr. Thomas Dresbach

Anatomy and Embryology, Göttingen University Medical School Center of Anatomy, Göttingen

Camin Dean, PhD

Trans-synaptic Signaling, European Neuroscience Institute, Göttingen

Day of oral examination: $18^{\text {th }}$ April 2013 

Herewith I declare that I prepared the PhD Thesis "The Role of PSD-95 and Kinase Interactions in Synaptic Transmission" on my own and with no other sources and aids than quoted.

Göttingen, 



\section{Abstract}

During development various structural and molecular changes take place in the brain leading to its maturation. These changes occur at multiple levels, including the alterations in protein expression level of single cells and in the synaptic strength of neuronal connections. PSD-95, one of the most important scaffold proteins, in excitatory synapses, was shown to be involved in some of these developmental processes. In this study, by using diverse electrophysiological and biochemical methods in combination with in vivo and in vitro lentiviral injection techniques, I examined the role of PSD-95 and its domains in the regulation of basal synaptic transmission and developmental NMDA receptor subunit switch.

NMDA receptor subunit switch is one of the most important events taking place during early postnatal development. The regulation of the GluN2B-containing NMDA receptor surface expression was proposed to involve PSD-95 and Src kinase interaction, and this interaction was shown to depend on the CDK5 phosphorylation state of PSD-95. However, the effect of this phosphorylation on synaptic transmission is unknown. In this study, I showed that a mutant form of PSD-95 mimicking the phosphorylated state enhanced both AMPAR and NMDAR transmission in a Src kinase- and GluN2B subunit-dependent manner. In addition, I could demonstrate that PSD-95 is indeed involved in the developmental NMDAR subunit switch in layer $2 / 3$ neurons of mouse visual cortex and that the expression of the phospho-mimicking mutant of PSD-95 in these neurons prevented the NMDAR subunit switch.

Besides the changes in the phosphorylation state, the importance of the SH3 and GK domains of PSD-95 was emphasized in the regulation of basal synaptic transmission. In order to dissect the specific roles of these domains, we generated molecular replacement constructs of PSD-95 lacking one or more of its domains. Expression of these constructs in CA1 region neurons of rat and PSD-95 knockout mouse hippocampus, demonstrated that the SH3 domain is necessary for creating a functional link between PSD-95 and SAP102, whereas GK domain in the absence of SH3 domain funcitons in a SAP102 independent way.

Overall, these data suggest a domain- and CDK5 phosphorylation state-specific effect of PSD-95 on the basal synaptic transmission of CA1 region neurons as well as a CDK5 phosphorylation state-specific effect of PSD-95 on the NMDAR subunit switch in layer $2 / 3$ neurons of visual cortex. These findings contribute to a better understanding of synaptic regulation mechanisms, hence of learning and memory. 


\section{Acknowledgements}

First, I would like to thank Dr. Dr. Oliver Schlüter for giving me the opportunity to do my $\mathrm{PhD}$ project in his lab. It was a great experience to share his enthusiasm and passion for science and also learn from his broad knowledge. I am very thankful for all the time invested in helpful discussions and also for his guidance throughout the project.

I would like to thank my thesis committee members Prof. Dr. Nils Brose and Dr. Till Marquardt for their comments and critics during the thesis committee meetings, which contributed to the development of my project.

I thank Prof. Dr. Michael Hörner, Dr. Camin Dean and Prof. Dr. Thomas Dresbach

for being a part of my extended thesis committee and taking part in this crucial stage of my $\mathrm{PhD}$.

Special thanks to the IMPRS office, Prof. Dr. Michael Hörner and Sandra Drube for their enormous help in all aspects ever since we arrived to Göttingen.

I would like to thank Christiane Becker for taking care of our contracts and making it easy for us to concentrate on our projects.

Many thanks to the GGNB office for organizing excellent seminar and excursions.

I am extremely grateful for all the amazing people I met in Göttingen!

I would like to thank my previous and present lab members, Juliane, Martina, Albrecht, Stephanie, Ania, Sandra, Yanling, Huang, Tanmoy, Plinio, Avani, Lei and Man Ho for creating a very joyful and friendly working environment in the lab. It was definitely a motivation to be a part of such a team!

Sandra, thank you very much for the incredible work you do and for your professional \& personal support! We are very lucky to have you.

Ania, I couldn't be happier to share my office with a person like you, who appreciates the warmth as much as I do! Also, thank you very much for the last minute help when I needed it and for happy teatime sessions.

I would like to thank Bekir for his help during the last months of my $\mathrm{PhD}$ and for his great personality, which was very fun to be around.

Mayur, thank you very much for your amazing friendship and for your never disappearing zen attitude! It was always a pleasure to spend time with you. 
My Göttingen 'Leute' group, Natalia, Steph, Alwaleed, Sanaz, Jonas, Andrea, Matthieu, Andrew, Samir, Chris, Liam, Alonso and Sünke, thank you guys so much for making this place so special! I gained invaluable friendships and memories during the last 5 years, which I deeply appreciate.

Jonas, thank you for your positive energy and for your open personality. Andrea, thanks for the great trip we had to Italy! It always amazed me how you could combine work with 'extracurricular activities' on a weekly even daily basis... Matthieu, it was without exception a pleasure to be involved in your german-french life style with sausage-fondue evenings accompanied by the Alsace wine. Andrew, thank you for the Thanksgiving and movie night gatherings, even though it was a miracle to pack so many people in a single room. Samir, thank you for the funny videos you shared with me. Alonso, I was very happy to have you as my dorm mate for two years, and Sünke, I had a great time at the festival we camped together! Chris, I hope at some point you will go back to your creative art projects. I definitely will wait for an opening invitation. Liam, although we 'met' a bit late, I enjoyed your company in all our meetings.

Sanaz, I am glad that you decided to move to Göttingen for your $\mathrm{PhD}$ so that we could meet each other! Thank you for the coffee meetings, sushi evenings and Hamburg trips. I am looking forward to many other occasions that are yet to come!

Steph, I don't know from where to start. Thank you for everything!!! For always being there no matter what, for being an amazing friend and a colleague, for your very positive attitude towards life and for everything else which I cannot fit in here... You were a big support during my $\mathrm{PhD}$ time.

My mini group, Natalia (my sis) and Alwaleed, thank you guys for being such a big part of my life and being a family to me. These 5 years wouldn't be the same without you!

Nikolai, thanks a lot for sharing all the important moments with me and adding more to them! Your support and encouragements motivated me a lot.

Lastly, I would like to thank my parents and my brother, Deniz, Uğursal and Tolga, for their unconditional love and support, which made everything easier! Iyi ki varsiniz! 


\section{Table of Contents}

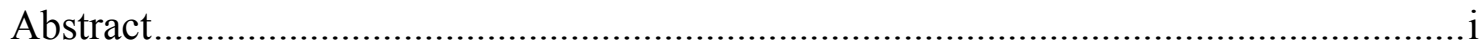

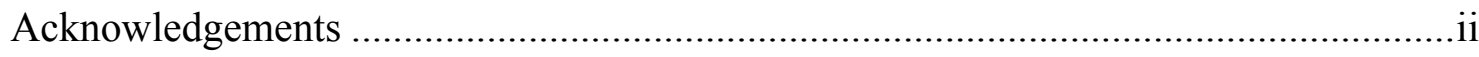

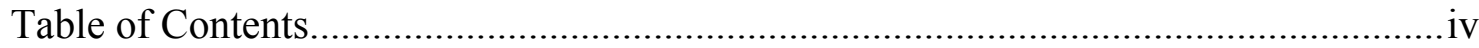

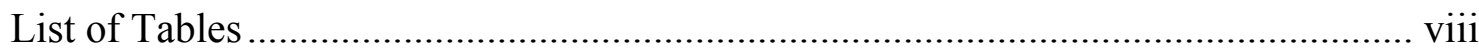

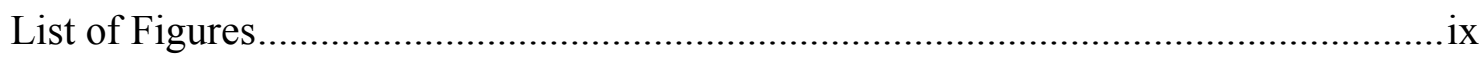

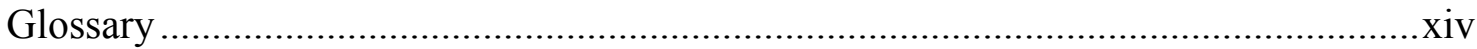

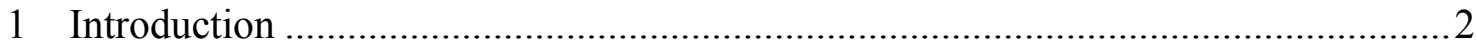

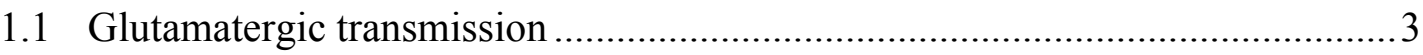

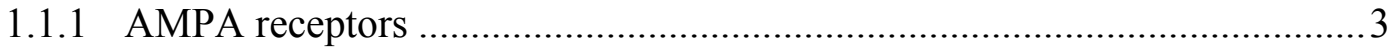

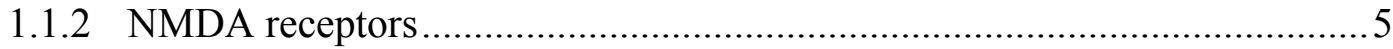

1.1.2.1 NMDA receptor-dependent synaptic plasticity ...............................6

1.1.2.2 NMDA receptor subunits and the developmental NMDA receptor

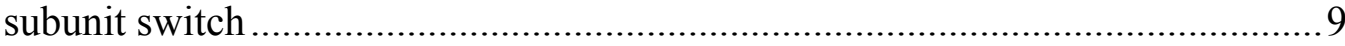

1.1.3 DLG-Membrane-associated guanylate kinase (MAGUK) family .............. 13

1.1.3.1 The role of DLG-MAGUKs in basal synaptic transmission and

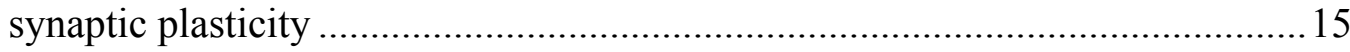

1.1.3.2 The role of PSD-95 in synaptic maturation and developmental NMDA

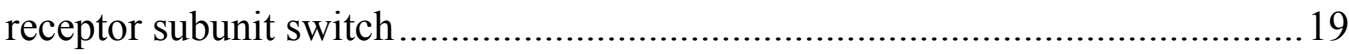

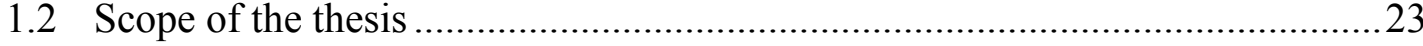

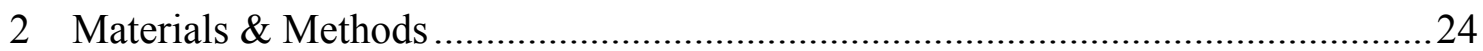

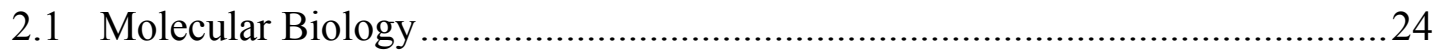

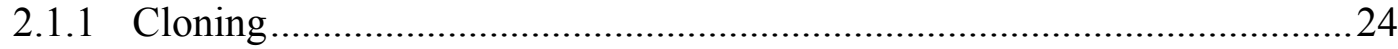

2.1.1.1 Polymerase chain reaction (PCR) ..............................................26

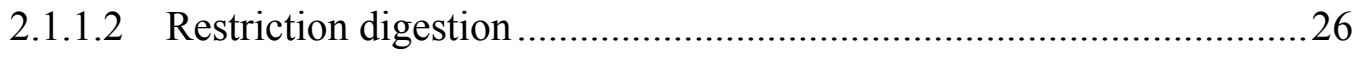

2.1.1.3 PCR product and agarose gel purification ...................................2 27

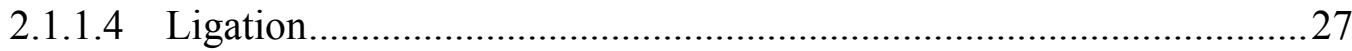

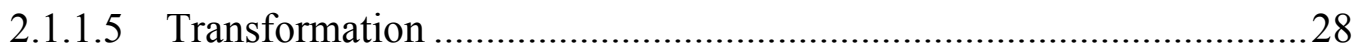

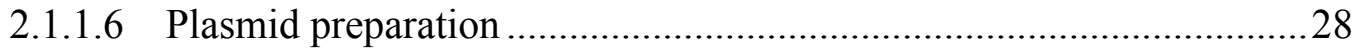

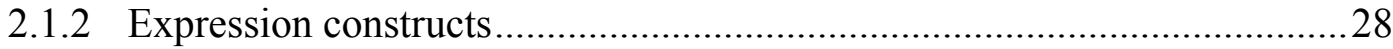


2.1.2.1 Generation of CDK5 mutants of PSD-95 as GFP fusion protein under the transcription enhancer WPRE cassette in lentiviral vector

2.1.2.2 Generation of CDK5 mutants of PSD-95 as GFP fusion protein without the WPRE enhancer cassette in lentiviral vector 32

2.1.2.3 Generation of CDK5 mutants of PSD-95 in bicistronic lentiviral vector with separate GFP expression via IRES cassette

2.1.2.4 Generation of dominant-negative CDK5, p25 and p35 overexpression constructs in lentiviral vector 34

2.1.2.5 PSD-95 domain mutants in lentiviral vector ..................................... 35

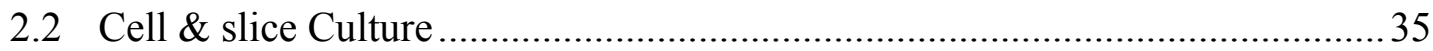

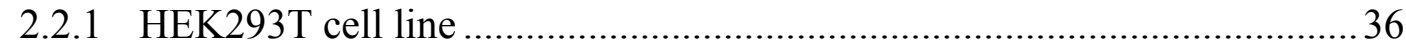

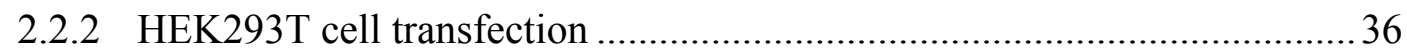

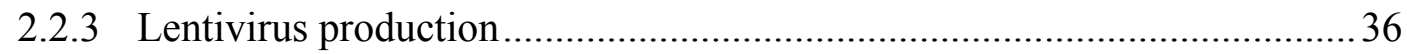

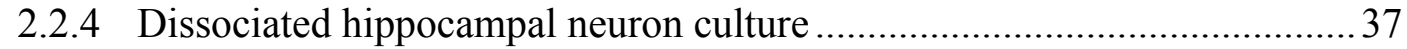

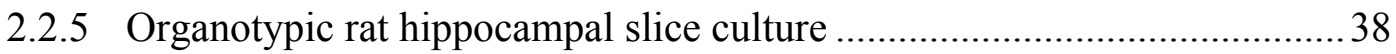

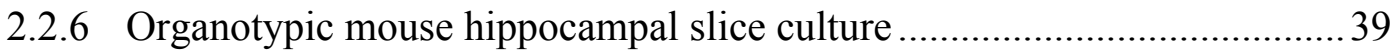

2.2.7 Acute mouse visual cortex slice preparation ...................................... 40

2.3 In vivo injection into mouse visual cortex ................................................. 40

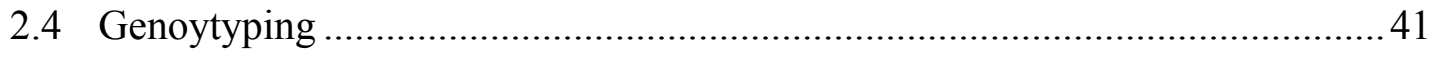

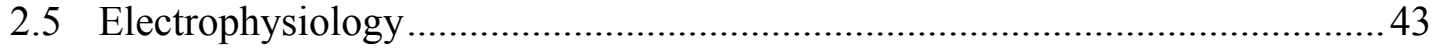

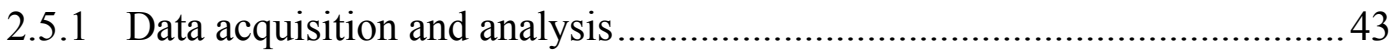

2.5.2 Basal synaptic transmission recordings in rat hippocampal slices ............44

2.5.3 Miniature EPSC recordings in rat hippocampal slices ........................... 45

2.5.4 NMDAR EPSC recordings in mouse visual cortex ............................... 45

2.5.5 Drugs used for pharmacological manipulations on hippocampal and brain

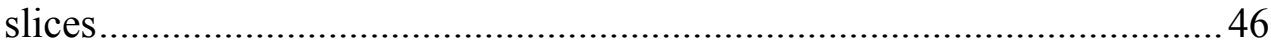

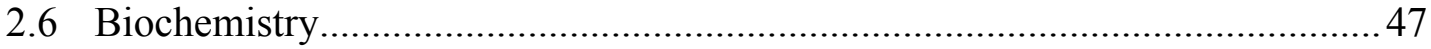

2.6.1 Dissociated neuron culture sample collection...................................... 47

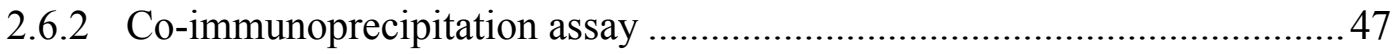

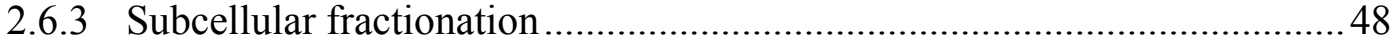

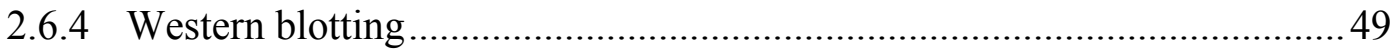


2.6.5 Antibodies used for Immunoblotting ………….....................................50

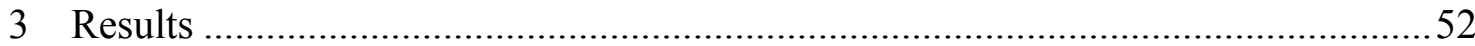

3.1 The effect of CDK5 phosphorylation mutants of PSD-95 on basal synaptic transmission in rat hippocampal slice culture ..................................................52

3.1.1 Optimization of the CDK5 phosphorylation mutant constructs of PSD-9552

3.1.2 AMPAR and NMDAR transmission properties of CDK5 mutants of PSD95 in CA1 region rat hippocampal neurons ................................................57

3.1.3 Activity manipulation of endogenous CDK5 in CA1 region neurons .........60

3.1.4 Involvement of Src kinase family in AMPAR and NMDAR transmission mediated by CDK5 phosphorylation mutants of PSD-95 ............................. 62

3.1.5 Activity-dependent effects of CDK5 phosphorylation mutants of PSD-95

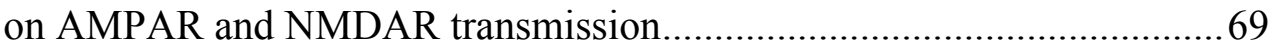

3.1.6 Pre- and postsynaptic properties of the CDK5 phosphorylation mutants of PSD-95 73

3.2 The role of CDK5 phosphorylation mutants of PSD-95 in the regulation of developmental NMDA receptor subunit switch in mouse visual cortex ...........76

3.3 Minimal PSD-95 to mediate the basal synaptic transmission and a molecular

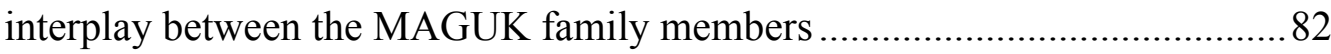

4 Discussion. 100

4.1 Important optimization steps in the generation of molecular replacement constructs 100

4.2 CDK5 phosphorylation site mutants of PSD-95 have different effects on AMPAR and NMDAR transmission..... 101

4.3 Endogenous CDK5 activity manipulation to confirm the mutation effects ....104

4.4 Src kinase family is involved in the regulation of AMPAR and NMDAR transmission mediated by CDK5 phosphorylation site mutants of PSD-95 ... 105

4.5 CDK5 phosphorylation site mutants of PSD-95 respond to activity changes differently 107

4.6 CDK5 phosphorylation mutants of PSD-95 regulate the developmental NMDAR subunit switch 110 
4.7 Minimal PSD-95 to regulate basal synaptic transmission in CA1 region of

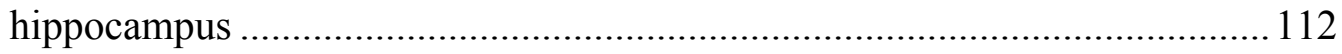

4.8 Compensation mechanism between the MAGUK members regarding the basal synaptic transmission in CA1 region neurons .......................................... 115

4.9 Molecular interplay between PSD-95 and SAP102 in CA1 region neurons of

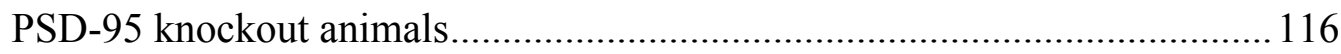

4.10 NMDAR transmission of minimal PSD-95 constructs ................................. 117

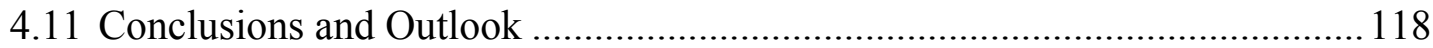

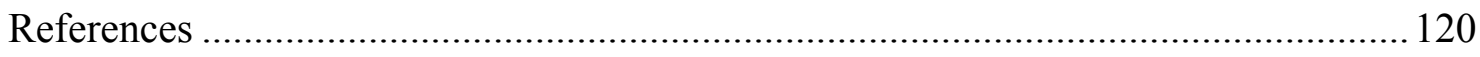

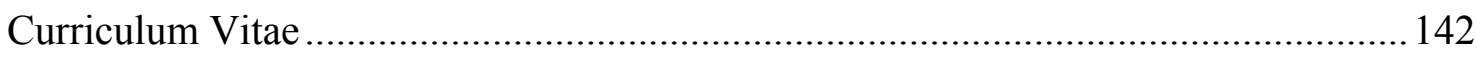




\section{List of Tables}

Table 1: The list of forward and reverse primers used for the cloning and sequencing of

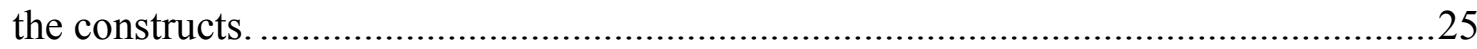

Table 2: Reaction mix for polymerase chain reaction. ..........................................26

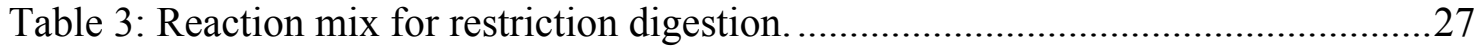

Table 4: List of the DNA constructs generated during the study. ..............................30

Table 5: DNA construct and amounts used for small and large scale virus production.37

Table 6: Primers used for wild-type and PSD-95 knockout animal genotyping...........41

Table 7: Content of the PCR mix used for wild-type and PSD-95 knockout animal

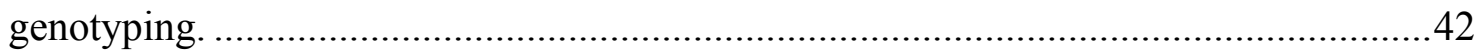

Table 8: PCR program used for wild-type and PSD-95 knockout animal genotyping. .42

Table 9: Equipment used for electrophysiological recordings. .................................43

Table 10: Concentrations, effects and the sources of the drugs used for

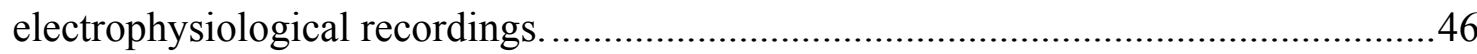

Table 11: Recipes of Bis-Tris gels used for Western-blotting. ..................................49

Table 12: List of the antibodies used for immunoblotting. ........................................51 


\section{List of Figures}

Figure 1: DLG-MAGUK family members.

Figure 2: A minimized sketch of PSD-95 interactions with synaptic receptors, ion channels and signaling molecules in the postsynaptic density.

Figure 3: Developmental NMDA receptor subunit switch observed in synapses.

Figure 4: Lentiviral vectors used for cloning. 29

Figure 5: CDK5 mutant constructs of PSD-95 with GFP-tag and enhanced expression.

Figure 6: CDK5 mutant constructs of PSD-95 with GFP-tag and without the enhancer effect.

Figure 7: CDK5 mutant constructs of PSD-95 with non-tagged GFP and endogenous levels of expression. 34

Figure 8: Overexpression constructs of dominant-negative CDK5 (DNK5), p25 and p35 in bicistronic lentiviral backbone.

Figure 9: In vivo injection applied on P0 mouse visual cortex.

Figure 10: Rat hippocampal slice culture preparation and recording scheme. .44

Figure 11: NMDAR EPSC recording in mouse visual cortex. .45

Figure 12: Expression of GFP-tagged CDK5 mutants of PSD-95 under the influence of WPRE transcription enhancer results in overexpression effect comparable to wild-type PSD-95 overexpression in CA1 region of rat hippocampus.

Figure 13: Expression of GFP-tagged CDK5 mutants of PSD-95 without the WPRE transcription enhancer distinguishes the mutation effect from the overexpression effect.

Figure 14: The C-terminal GFP-tag of PSD-95 interferes with the PSD-95 - Src kinase interaction. .56

Figure 15: Expression levels of CDK5 phosphorylation mutants of PSD-95 in dissociated neuron culture extracts.

Figure 16: The AMPAR and NMDAR transmission is significantly increased with the non-tagged phospho-mimicking mutant of PSD-95 under endogenous levels of expression. 
Figure 17: Overexpression of CDK5 co-activators p25 and p35 and a dominantnegative form of CDK5 (DNK5) do not alter the AMPAR and NMDAR transmission in CA1 region neurons

Figure 18: Src kinase family members are involved in the significant increase of AMPAR and NMDAR transmission observed with the phospho-mimicking mutant of PSD-95 in CA1 region neurons.

Figure 19: The increase in NMDAR response observed with phospho-mimicking mutant is GluN2B subunit specific in CA1 region neurons. .64

Figure 20: Phospho-mimicking mutant has a tendency to enhance Y1472 phosphorylation on GluN2B without changing total levels of GluN2B.

Figure 21: Activation of PAC1Rs, known to activate Src kinase, brings the NMDAR transmission of phospho-mimicking mutant expressing cells to the same level as control cells in CA1 region neurons. .....

Figure 22: Activation of D1 dopamine receptors, known to activate Fyn kinase, enhances both AMPAR and NMDAR transmission of non-phosphorylatable mutant expressing cells, at the same time bringing the AMPAR and NMDAR transmission of control cells to the same level as phospho-mimicking mutant expressing cells in CA1 region neurons. .68

Figure 23: The significant increase in AMPAR transmission of phospho-mimicking mutant expressing cells is not due to the enhancement in NMDAR transmission in CA1 region neurons.

Figure 24: Blockade of the slice activity for two days by TTX treatment enhances the effect of phospho-mimicking mutant of PSD-95 on AMPAR transmission in CA1 region neurons. .71 Figure 25: Blockade of mGluR5 activity in the slices for two days enhanced the AMPAR transmission of non-phosphorylatable mutant while eliminating the increase in NMDAR responses normally observed with phospho-mimicking mutant in CA1 region neurons.

Figure 26: CDK5 phosphorylation mutants of PSD-95 do not alter the frequency of miniature EPSCs in CA1 region neurons. .74 
Figure 27: No difference was observed between the paired-pulse ratio of mutant expressing and control cells in CA1 region neurons. .75

Figure 28: Non-phosphorylatable mutant of PSD-95 does not affect the extra-synaptic AMPA receptor pool in CA1 region neurons. .76

Figure 29: The contribution of GluN2B-containing NMDA receptors to NMDAR responses is reduced after eye opening in visual cortex later $2 / 3$ neurons. 78 Figure 30: PSD-95 knockout animals are deficient in establishing the NMDA receptor subunit switch upon eye opening in mouse visual cortex layer $2 / 3$ neurons..... .79 Figure 31: PSD-95 is involved in the NMDAR subunit switch in mouse visual cortex.

Figure 32: Phospho-mimicking mutant of PSD-95 prevents the NMDAR subunit switch upon eye opening

Figure 33: PSD-95 replacement increases AMPAR responses while knock-down of endogenous PSD-95 or replacement of it by PDZ1 $\backslash 2:$ GFP cause a significant decrease in AMPAR transmission in CA1 region rat hippocampal neurons.

Figure 34: SH3 domain of PSD-95 is dispensable for the regulation of basal synaptic transmission in CA1 region of rat hippocampus, whereas the role of PDZ3 and GK domains is not clear.

Figure 35: PSD-95 lacking the GK domain is functional and the addition of GK domain to PDZ1/2::GFP does not fully rescue the AMPAR transmission loss generated by PDZ1/2::GFP expression in CA1 region rat hippocampus.

Figure 36: PSD-95 lacking the GK domain is functional in CA1 region neurons of mouse hippocampal slice culture 89

Figure 37: Full-length PSD-95 expression increases AMPAR responses in infected cells by 2.5 fold in CA1 region hippocampal PSD-95 knockout slices, whereas PDZ1/2::GFP expression does not enhance the AMPAR transmission. .90 Figure 38: PSD-95 mutants lacking PDZ3 or GK domain enhance AMPAR mediated transmission in PSD-95 knockout background without altering the NMDAR transmission. .91

Figure 39: SAP102 and SAP97 levels are elevated in the PSD fraction of PSD-95 knockout animals 
xii

Figure 40: The AMPAR response enhancement of cells expressing GK domain lacking PSD-95 in PSD-95 knockout background is SAP102 dependent................................94 Figure 41: The function of PSD-95 lacking the PDZ3 domain, but not the function of PDZ1/2-GK::GFP protein is SAP102 dependent in the CA1 region of PSD-95 knockout

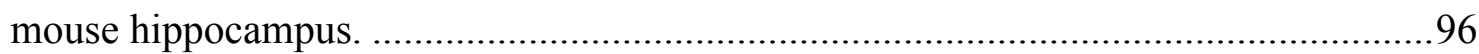
Figure 42: A minimal PSD-95 with the first two PDZ domains and the SH3 domain enhances basal synaptic transmission in CA1 region PSD-95 knockout neurons in a

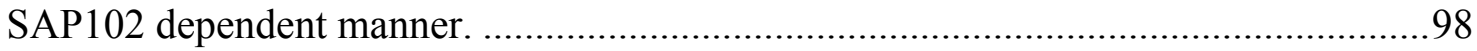




\section{Glossary}

ACSF

AEO

AMPAR

BEO

BSA

CamKII

cAMP

CDK5

CKII

D1

DIV

DL-TBOA

DLG-MAGUK

DNK5

EPSC

GABA

GFP

GK

GRIP

IRES

JNK

KO

LTD

LTP

MAPK/ERK
Artificial cerebrospinal fluid

After eye opening

Alpha-amino-3-hydroxyl-5-methyl-4-isoxazole-propionate receptor

Before eye opening

Bovine serum albumin

Calmodulin-dependent kinase II

Cyclic adenosine monophosphate

Cyclin-dependent kinase 5

Casein-kinase II

Dopamine-1

Day in vitro

DL-threo- $\beta$-Benzyloxyaspartic acid

Drosophila melanogaster Discs-large - Membrane associated guanylate kinase

Dominant-negative CDK5

Excitatory postsynaptic current

Gamma-aminobutyric acid

Green fluorescent protein

Guanylate kinase

Glutamate receptor interacting protein

Internal ribosome entry site

c-Jun NH2-terminal kinase

Knockout

Long-term depression

Long-term potentiation

Mitogen-Activated Protein Kinase / extracellular-signalregulated kinase 
mEPSC

mGluR

NBQX

NMDAR

P x

PAC1R

PACAP

PCR

PDZ

PICK1

PKA

PKC

PLC

PSD

PSD-93

PSD-95

RNAi

SAP102

SAP97

SDS-PAGE

SEM

SH3

ShRNA

STEP

TARP

TTX

WPRE

Wt
Miniature excitatory postsynaptic current

Metabotropic glutamatergic receptor

2,3-Dioxo-6-nitro-1,2,3,4-tetrahydrobenzo[f]quinoxaline-7-

sulfonamide

$\mathrm{N}$-methyl-D-aspartate receptor

Postnatal day $\mathrm{x}$

Pituitary adenylate cyclase activating polypeptide type 1 receptor

Pituitary adenylate cyclase-activating polypeptide

Polymerase chain reaction

PSD-95/Discs large/zona occludens-1

Protein interacting with $\mathrm{C}$ kinase 1

Protein kinase A

Protein kinase $\mathrm{C}$

Phospholipase C

Postsynaptic density

Postsynaptic density protein-93

Postsynaptic density protein-95

Interference RNA

Synapse-associated protein 102

Synapse-associated protein 97

SDS-polyacyrilamide gel

Standard error of the mean

Src-homology domain 3

Short-hairpin RNA

Striatal-Enriched tyrosine Phosphatase

Transmembrane AMPAR regulatory protein

Tetradotoxin

Woodchuck Hepatitis Virus (WHP) Posttranscriptional

Regulatory Element

Wild-type 


\section{Introduction}

Starting with the first written evidence about the nervous system in $1600 \mathrm{BC}$, Edwin Smith papyrus (Elsberg, 1931; Kamp et al., 2012), many scientists wondered about the function of the brain. While extensive efforts are invested each year into brain research and to find out possible ways to cure its malfunction, the biggest question of how the brain works is not yet fully answered.

Despite the large gaps we need to fill for a complete understanding, there is a considerable amount of data regarding how 75 million neurons in a mouse brain, respectively 100 billion neurons in a human brain can communicate with each other.

Until now, we have evidence that neurons communicate with each other through electrical and chemical means. The structures where the information exchange takes place are called synapses. Electrical synapses are formed by gap junctions which are intercellular channels composed of connexon molecules (Goodenough et al., 1996). Gap junctions connect the plasma membranes of two adjacent cells and allow the passage of ions as well as molecules smaller than $1.5 \mathrm{~nm}$ such as $\mathrm{Ca}^{2+}$ and secondary messengers like cyclic AMP. Exchange of these molecules results in a direct coupling between two neurons, synchronizing their act (Bennett and Zukin, 2004). Chemical synapses, on the other hand, are structures where the plasma membranes of two neurons are closely positioned to each other with a separation of 20-30 nm. This space between the pre and postsynaptic neuron is named as synaptic cleft and allows the successful diffusion of neurotransmitter molecules which are the essence of synaptic communication in neuronal systems. Neurotransmitters are classified as excitatory, inhibitory and modulatory depending on their action on selected receptor types. In the brain, L-glutamate is considered to be the most abundant excitatory neurotransmitter, whereas $\gamma$-Aminobutyric acid (GABA) is known to be the main inhibitory neurotransmitter. Serotonin, dopamine and noradrenaline on the other hand, are some of the neurotransmitters known for their modulatory effect on neuronal function (Panula et al., 2006).

Neurotransmitters are stored in synaptic vesicles at the presynaptic terminal and are released into the synaptic cleft in a $\mathrm{Ca}^{2+}$-dependent manner after a depolarizing event 
(McMahon and Nicholls, 1991). This release triggers the activation of neurotransmitterspecific receptors on the postsynaptic membrane. Postsynaptic receptors can trigger different actions depending on the receptor type, either mediating ion exchange between the extra-cellular matrix and the cytoplasm (ionotropic receptors) or activating molecules to manipulate down-stream secondary messenger pathways (metabotropic receptors). Both of these actions are crucial to maintain the connections in a neuronal network as well as to change the strength of existing connections in different brain regions which underlies the mechanism of long-term plasticity, a cellular model of learning and memory (Bliss and Lomo, 1973; Komatsu and Iwakiri, 1993; Malenka and Nicoll, 1993; Greenamyre and Porter, 1994; Stelzer et al., 1994; Malinow and Malenka, 2002).

\subsection{Glutamatergic transmission}

Excitatory transmission is one of ways to keep a neuronal system communicating, besides the inhibitory and modulatory transmission. In the brain, most of the excitatory transmission is mediated through L-glutamate. Its action is exerted on four types of glutamate receptors, which are located on the postsynaptic membrane. These receptors include three types of ionotropic, $\alpha$-amino-3-hydroxy-5-methyl-4-isoxazolepropionic acid (AMPA) receptors, $N$-methyl-D-aspartate (NMDA) receptors, kainate receptors and different metabotropic glutamate receptors (mGluRs) (Watkins et al., 1990; Nakanishi, 1992; Dingledine et al., 1999). In this study, I will focus on the function of AMPA and NMDA receptors.

\subsubsection{AMPA receptors}

AMPA receptors are tetrameric entities composed of four subunits, GluA1-4 (Wisden and Seeburg, 1993; Hollmann and Heinemann, 1994). The subunits differ from each other primarily by the structure of their C-termini. GluA1 and 4 subunits have longer C-termini whereas GluA2 and 3 have shorter C-terminal tails (Song and Huganir, 2002). The combination of different subunits determines the functional properties of the receptors. These properties can range from specific protein-protein interactions (Gardner et al., 2005) and phosphorylation sites (Roche et al., 1996; Carvalho et al., 
1999; Matsuda et al., 1999) to $\mathrm{Ca}^{2+}$ permeability and rectification of the receptors (Hayashi et al., 2000; Song and Huganir, 2002; Kakegawa et al., 2004). The two mechanisms determining the subunit structure are governed by the post-transcriptional Q/R mRNA editing (Seeburg, 2002) and also by the alternative splicing mechanism (Sommer et al., 1990). The majority of AMPA receptors are formed by GluA1 and GluA2 or by GluA2 and GluA3 heteromeres. The GluA2 subunit confers $\mathrm{Ca}^{2+}$ impermeability due to the post-transcriptional $\mathrm{Q} / \mathrm{R}$ editing and also provides linear rectification for the channels whereas GluA2-lacking receptors are $\mathrm{Ca}^{2+}$ permeable and have higher rectification (Burnashev et al., 1992). This difference is considered to be crucial for the type of fast excitatory transmission that a synapse conveys. The composition of synaptic AMPA receptors change from dominantly GluA2-lacking to GluA2-containing receptors over the course of early development in rat neocortex (Kumar et al., 2002) as well as in response to the changes in the strength of synaptic transmission (Plant et al., 2006).

The role of AMPA receptors is intensively studied both in basal synaptic transmission and different plasticity forms such as long-term potentiation and long-term depression, which are suggested to be the underlying mechanisms of learning and memory processes. Both of these processes demand accurate targeting and maintenance of AMPA receptors at the synaptic membrane.

Synaptic AMPA receptor are located in the postsynaptic density (PSD) of excitatory neurons, which is a $30-40 \mathrm{~nm}$ thick electron-dense structure underneath the postsynaptic membrane (Ziff, 1997; Scannevin and Huganir, 2000; Feng and Zhang, 2009). PSD is organized as a network of ion channels, scaffold proteins and signaling pathway elements. Among various binding partners, AMPA receptors are known to directly interact with transmembrane AMPAR regulatory proteins (TARPs) in the postsynaptic density of excitatory synapses (Chen et al., 2000; Tomita et al., 2005). This enables the functional connection between AMPA receptors and postsynaptic scaffold proteins, one of the most important being postsynaptic density 95 (PSD-95) protein. The interaction between AMPARs and PSD-95 is known to be crucial for AMPA receptor trafficking at the synapse (El-Husseini et al., 2000c; Béïque et al., 2006; Elias et al., 2006). Moreover, AMPA receptor function and trafficking can be 
modulated further by the binding of various proteins such as, protein interacting with $\mathrm{C}$ kinase 1 (PICK1) (Terashima et al., 2004; Jaafari et al., 2012) and glutamate receptor interacting protein (GRIP) (Yamazaki et al., 2001; Lu and Ziff, 2005).

Besides their function in basal synaptic transmission, changes in the AMPA receptor number is one of the key regulations in the establishment of long-term plasticity. In this regard, two important kinases were shown to modulate the function of AMPA receptors and thus modulate their contribution to the synaptic plasticity. The first kinase, protein kinase A (PKA), phosphorylates the Ser845 residue on GluA1 subunit and triggers the insertion of GluA1 containing AMPA receptors to the postsynaptic membrane (Malinow, 2003; Man et al., 2007). The second kinase, wellknown for its action in long-term potentiation is the calcium-calmodulin dependent kinase II alpha (CamKII). CamKII phosphorylates Ser831 and increases the single channel conductance of GluA1 homomeres (Barria et al., 1997; Lee et al., 2000).

In addition to the aforementioned control mechanisms, other pathways and proteins orchestrate the AMPA receptor synthesis, function and transportation, hence the transmission through a single synapse. These mechanisms may involve the activation of distinct receptors such as D1/D5 dopamine (Smith et al., 2005), NMDA (Delgado et al., 2007) or metabotropic glutamate receptors (Kelly et al., 2009; Casimiro et al., 2011; Sanderson et al., 2011) to regulate the synthesis of AMPA receptors directly, or the manipulation of other pathways and kinases such as MAPK/ERK (Grooms et al., 2006) and Protein kinase C (PKC) (Boehm et al., 2006) to control the AMPA receptor function.

\subsubsection{NMDA receptors}

With their involvement in the induction of long-term plasticity, in the regulation of basal synaptic transmission and in pathological brain disorders, NMDA receptors have been a major focus of research in the last 30 years (Watkins, 1981).

NMDA receptors are cationic channels allowing the flow of $\mathrm{Na}^{+}, \mathrm{K}^{+}$and $\mathrm{Ca}^{2+}$ ions through their channel pores. The ability to transmit $\mathrm{Ca}^{2+}$ ions upon a stimulation gives them the exceptional feature to control and contribute to many regulatory pathways in the postsynaptic side of a neuron, including increasing and decreasing the strength of an 
existing synapse. As AMPA receptors, NMDA receptors are also composed of four subunits, GluN1-4 (Nakanishi, 1992; Hollmann and Heinemann, 1994). Two GluN1 subunits being obligatory for all the receptors (Fukaya et al., 2003), differentially combined with GluN2, 3 and 4 subunits can result in the formation of di- or triheteromeric channels. The combination of these different subunits assigns specific characteristics to each channel (Monyer et al., 1992). GluN2 is the subunit responsible of binding the excitatory neurotransmitter glutamate and is essential for the regulation of channel gating by mediating the $\mathrm{Mg}^{2+}$ blockade and $\mathrm{Ca}^{2+}$ permeability (Mayer and Armstrong, 2004). On the other hand, the GluN1 subunit is necessary for the ion selectivity and binding the co-agonists glycine and D-serine (Cull-Candy and Leszkiewicz, 2004). In addition to the GluN2 subunit, GluN3 also has the ability to bind glycine. Thus the combination of GluN1 and 3 subunits can render the channel $\mathrm{Ca}^{2+}$ impermeable (Henson et al., 2010).

In order to be activated, NMDA receptors rely on the mechanism called "coincidence detection". This requires the simultaneous triggering of presynaptic release of glutamate and the depolarization of the postsynaptic membrane. Only then, the $\mathrm{Mg}^{2+}$ block within the channel pore is relieved, and the flux of cations is established (Mayer et al., 1984; Nowak et al., 1984). The following influx of calcium ions is the main signal to initiate NMDA receptor-dependent synaptic plasticity.

\subsubsection{NMDA receptor-dependent synaptic plasticity}

The first model to understand learning and memory formation was proposed in 1973, when Bliss and Lomo triggered an increase in the synaptic responses of dentate gyrus neurons following a tetanic stimulation (Bliss and Lomo, 1973). The phenomenon was termed as long-term potentiation and since then was taken as an exciting model to underlie information storage in neurons which was correlated with the activitydependent changes in synapses (Hebb, 1949). Following this study, more evidence was collected over time, indicating that in CA1 region of hippocampus, as well as in some other brain regions, the induction of long-term plasticity is NMDA receptor dependent (Cull-Candy and Leszkiewicz, 2004; Malenka and Bear, 2004; Rebola et al., 2010). These information made the NMDA receptor-dependent plasticity the most extensively 
studied plasticity form in order to understand the molecular mechanisms underlying learning and memory, particularly in hippocampus, a brain region which is believed to acquire new memory traces (Zola-Morgan et al., 1986; Rempel-Clower et al., 1996). The formation of new memories is established by generating new synaptic connections or by changing the strength of the already existing ones within a neuronal network. The process for mediating such a change is called synaptic plasticity. Long-term plasticity is classified into long-term potentiation (LTP) and long-term depression (LTD), which respectively result in the strengthening or weakening of the synaptic connections between the neurons. This alteration in the synaptic strength is achieved through the change in the postsynaptic $\mathrm{Ca}^{2+}$ concentration via NMDA receptor activation. The elevation in the $\mathrm{Ca}^{2+}$ levels at the postsynapse modulates the activity of various kinases such as CamKII, PKA, MAPK and PKC, or phosphatases such as protein phosphatase 2B (PP2B) to eventually alter the function and/or number of AMPA receptors (Lisman, 1994; Rebola et al., 2010). Most known changes on AMPA receptors following LTP and LTD inductions are the phosphorylation state of S831 and S845 on GluA1 subunit. While LTP induction favors the phosphorylation of these residues by CamKII and PKA, LTD induction eliminates the phosphorylations through phosphatase activity (Kessels and Malinow, 2009). However, altering the phosphorylation state of GluA1 subunit is not sufficient to explain the long-term plasticity mechanisms, since mice with a knockin of S831 phosphorylation deficient GluA1 exhibited normal LTP and LTD, and mice with the knock-in of S845 phosphorylation deficient GluA1 was deficient only in LTD (Lee et al., 2010).

In addition to the manipulations of the AMPA receptor function and number, many studies within the last 20 years pointed out activity-dependent modifications on synaptic NMDA receptors following different LTP protocols in hippocampal areas (Bashir et al., 1991; Berretta et al., 1991; Kwon and Castillo, 2008; Rebola et al., 2008). Not surprisingly, several kinases indicated in long-term potentiation mechanisms to alter the AMPA receptor function, also phosphorylate and bind to NMDA receptors. One such example is the phosphorylation of both GluN1 and GluN2 subunits of NMDA receptors by PKC. The GluN1 subunit is phosphorylated on two serine residues, S890 and S896, which regulate the surface expression and the clustering of the 
receptors (Tingley et al., 1997; Scott et al., 2003). On the other hand, the GluN2A subunit has two potential phosphorylation sites, S1291 and S1312, to increase the conductance of the GluN2A-containing receptors (Gardoni et al., 2001; Grant et al., 2001; Jones and Leonard, 2005). A similar potentiation was observed with GluN2Bcontaining receptors when S1303 and S1323 residues on GluN2B subunit were phosphorylated by PKC (Liao et al., 2001).

Besides PKC, CamKII is the second kinase indicated with its ability to bind GluN2 subunits A and B. However, the affinity of CamKII to bind GluN2B is much higher than binding GluN2A subunit (Bayer et al., 2001). The binding itself and its specificity are crucial steps in the maintenance of LTP at the synapses (Barria and Malinow, 2005; Sanhueza et al., 2011; Halt et al., 2012). In both cases, a cross-talk between PKC and CamKII pathways is suggested to regulate the CamKII binding to GluN2A and B subunits. It was reported that CamKII phosphorylates S1303 residue on the GluN2B subunit, which also is a substrate of PKC. Phosphorylation of this serine residue alters the strength of CamKII and GluN2B binding (Strack et al., 2000; Liu et al., 2006; Raveendran et al., 2009). In a similar way, the interaction between alpha-CamKII and GluN2A subunit is perturbed if the S1416 residue in the CamKII binding region of GluN2A is phosphorylated by PKC (Gardoni et al., 2001).

Further kinases, such as PKA, CDK5 and CKII, were implicated with their role in the phosphorylation of NMDA receptor subunits and thus the regulation of the receptor function (Chen and Roche, 2007). PKA activity was shown to increase the synaptic targeting, $\mathrm{Ca}^{2+}$ permeability and also the conductance of NMDA receptors (Raman et al., 1996; Crump et al., 2001; Skeberdis et al., 2006). A rather subunit-specific action was proposed through CDK5 and CKII activity. While CDK5 phosphorylates only GluN2A subunit and enhances the transmission (Li et al., 2001), CKII specifically phosphorylates the S1480 residue on GluN2B and results in the disruption of PSD-95 and NR2B interaction (Chung et al., 2004). The regulation of PSD-95-GluN2B interaction through CKII is important for the clustering of GluN2B-containing NMDA receptors at the synapse (Sanz-Clemente et al., 2010).

Finally, a family of non-receptor tyrosine kinases, the Src kinase family, was brought to light with its substantial role in regulating NMDA receptor surface 
expression through the phosphorylation of several residues on GluN2 subunits. The family consists of five members (Src, Fyn, Lyn, Lck, and Yes) all of which are present in the PSD of glutamatergic synapses (Kalia et al., 2004). However, among the family members, two particular kinases, Src and Fyn, received most attention and were studied extensively for their regulatory actions on NMDA receptors. This interest was triggered by the fact that both of the kinases could be extracted within the synaptic NMDAR complex (Yu et al., 1997; Yaka et al., 2002). Src kinase was shown to phosphorylate Y1292, Y1325 and Y1387 residues on GluN2A subunit, which results in the enhancement of NMDAR transmission (Köhr and Seeburg, 1996; Zheng et al., 1998). Additionally, the phosphorylation of Y842 residue was pointed to regulate the surface expression of GluN2A-containing receptors via preventing the interaction with AP-2 complex, the core component of clathrin-mediated endocytosis (Vissel et al., 2001). On the other hand, Fyn kinase was reported to phosphorylate Y1252, Y1336 and Y1472 residues on GluN2B subunit (Nakazawa et al., 2001; Takasu et al., 2002). Particularly, Y1472 is an important site to control GluN2B-containing NMDA receptor surface expression, as it is located in the internalization motif, YEKL. Phosphorylation of this site prevents the binding of AP-2 adaptor complex hence enhances the surface expression of GluN2B-containing NMDAR's (Roche et al., 2001). A recent study also suggested the phosphorylation of Y1472 residue by Src kinase, again controlling the surface expression of the receptors (Zhang et al., 2008). Tyrosine phosphorylation on NMDA receptors is at the same time regulated and balanced by the activity of specific protein tyrosine phosphatases. Striatal enriched tyrosine phosphatase (STEP), as an example, is involved in the dephosphorylation of the Y1472 site on GluN2B and promotes the endocytosis of the GluN2B-containing receptors (Snyder et al., 2005). Such interplay between the tyrosine kinases and phosphatases is vital to regulate the NMDA receptor function, as a consequence control the synaptic plasticity.

\subsubsection{NMDA receptor subunits and the developmental NMDA receptor subunit switch}

It is well documented that the expression of NMDA receptor subunits changes in a spatio-temporal manner in the brain. GluN1 subunit is expressed before and after birth 
ubiquitously throughout the brain, whereas GluN2 subunit isoforms have a rather specific distribution and expression profile. GluN2B and GluN2D subunits are present in the embryonic stage. In contrast, GluN2A and GluN2C subunits appear in different brain regions postnatally. While the GluN2B expression in the adult brain is enriched in the forebrain, GluN2C is highly abundant in the cerebellum and GluN2D expression shifts to spinal cord in later developmental stages (Monyer et al., 1994). GluN3 subunits also differ in their expression levels, GluN3A being more prominent in the early development and GluN3B increasing through late developmental stages (Henson et al., 2010).

Among different NMDA receptor subunits and their diverse roles, GluN2A and GluN2B subunits got into the spotlight of research due to their significant functional contribution to various plasticity forms, such as long term plasticity in several brain areas (Dickenson and Sullivan, 1991; Szinyei et al., 2003; Foster et al., 2010), metaplasticity (Yashiro and Philpot, 2008) and ocular-dominance plasticity (Fagiolini et al., 2003); developmental processes, as in cortical development (Wang et al., 2011) and hippocampal synaptogenesis (Gambrill and Barria, 2011); also via their act in several neurodegenerative disorders, for instance in Alzheimer's disease (Snyder et al., 2005; Wu and Hou, 2010; Rönicke et al., 2011), Schizophrenia (Gaspar et al., 2009) and Parkinson's disease (Dunah et al., 2000), just to mention a few.

The uniqueness of NMDA receptor subunits to play a role in different processes comes from their functional and kinetic properties as well as the set of specific proteins they interact with. The very first characteristic, distinguishing GluN2A and GluN2B subunits, is the kinetic features they give to the receptors. The GluN2A subunit demonstrates faster kinetics with respect to the open probability (Erreger et al., 2005) and the deactivation time constant. GluN2B-containing receptors deactivate with a constant of $250 \mathrm{~ms}$, whereas GluN2A-containing NMDA receptors deactivate with a constant of $100 \mathrm{~ms}$ (Cull-Candy and Leszkiewicz, 2004). This difference in kinetics is one of the important factors determining the response of the neurons to synaptic stimuli. In addition to the open probability and deactivation time constant, the subunits can be differentially blocked by specific inhibitors. Ifenprodil was shown to specifically block GluN2B-containing receptors with an $\mathrm{IC}_{50}$ of $0.3 \mu \mathrm{M}$ whereas the same inhibitor gives 
an $\mathrm{IC}_{50}$ value of $146 \mu \mathrm{M}$ for GluN1/GluN2A receptors (Williams, 1993; Bhatt et al., 2013). In contrary, Zinc was indicated to be specific GluN1/GluN2A receptor blocker with its biphasic inhibition profile and voltage dependence, with $\mathrm{IC}_{50}$ values of $80 \mathrm{nM}$ to $79 \mu \mathrm{M}$ depending on the high- and low-affinity phase. However for GluN1 $\backslash$ GluN2B receptors, $\mathrm{IC}_{50}$ values were $9 \mu \mathrm{M}$ (Williams, 1996; Paoletti et al., 1997).

Regarding the requirement of co-agonists to activate NMDA receptors, a recent article reported a binding preference of GluN2A and 2B subunits to different coagonists. The study suggested high affinity binding of GluN2B to glycine and GluN2A to D-serine (Papouin et al., 2012). The usage of these specific blockers and co-agonists during synaptic stimulation, led to further observations that NMDA receptors localize in a subunit-specific manner around the synapse. Even though previous studies suggested a strict synaptic localization for GluN2A and extra-synaptic localization for GluN2B-containing receptors (Stocca and Vicini, 1998; Mohrmann et al., 2000; Tovar et al., 2000; Townsend et al., 2003), follow-up studies showed the presence of both subunits in both compartments, still preserving the hypothesis of GluN1/GluN2A receptors mainly cluster at the synaptic site whereas GluN1/GluN2B receptors accumulate in the extra-synaptic site (Thomas et al., 2006; Papouin et al., 2012). In addition, GluN2B-containing receptors are more mobile in the synapse in comparison to the receptors with GluN2A subunit (Groc et al., 2006b).

Another important feature of GluN2A and GluN2B subunits is the difference in their expression profile during the development. Various studies conducted in different brain regions demonstrated a developmental NMDA receptor subunit switch, which is an activity-dependent process (Dumas, 2005). The occurrence of this switch within the critical period makes it an attractive topic to study activity-dependent synaptic plasticity forms, which involve NMDA receptors.

One of the first evidence to report the change in NMDA receptor subunit composition during development was gathered from the experiments done on rat cortex, and demonstrated that the GluN2B subunit is already present in the cortical neurons at birth whereas the expression of GluN2A subunit increases during the first two weeks of development (Sheng et al., 1994). Electrophysiological properties of cortical neurons also changed accordingly, showing faster kinetics and ifenprodil sensitivity over the 
course of development (Hestrin, 1992; Flint et al., 1997; Tovar and Westbrook, 1999). Additional studies contributed to the existence of this phenomenon. A similar switch was observed in mouse thalamic and cortical synapses during early development, where GluN2A subunit expression elevated until the post-natal day 15 (Liu et al., 2004b). A relatively recent study by Bellone \& Nicoll suggested an activity-dependent bidirectional switch of the NMDA receptor subunits in the hippocampus of young rats (Bellone and Nicoll, 2007). Application of the LTP protocol resulted in faster kinetics and less ifenprodil sensitivity of NMDA receptors. Accordingly, depotentiating the synapses slowed down the decay of the NMDAR excitatory postsynaptic currents (EPSCs) while increasing the ifenprodil sensitivity. Another study examined the change in NMDAR subunit composition thus their contribution to long-term synaptic plasticity in rat organotypic hippocampal slice culture system (Foster et al., 2010). In consistency with the hippocampal study by Bellone \& Nicoll, a bidirectional change in NMDA receptor subunit was observed in layer 2/3 neurons of rat visual cortex upon visual stimulation (Philpot et al., 2001). Visual experience resulted in a decrease of the GluN2B involved currents, whereas visual deprivation enhanced the GluN2B contribution.

Even though the existence of such a subunit switch is reported in several cases, the exact mechanism mediating the switch itself is not well understood. However, it is possible that the subunit-specific localization as well as the developmental expression profile of GluN2 subunits are correlated with the distinctness of their interacting partners.

Among the various proteins that NMDA receptors interact with, membraneassociated guanylate kinase (MAGUK) family members are special with their role in the regulation of NMDA receptor function and clustering at the synapse. Their ability to directly interact with NMDA receptors and link them to down-stream molecular pathways renders them potential candidates for mediating such a switch. 


\subsubsection{DLG-Membrane-associated guanylate kinase (MAGUK) family}

Drosophila melanogaster Discs-large (DLG)-MAGUKs, a subcategory of synaptic membrane-associated proteins, are the major scaffolding proteins found in the PSD of excitatory synapses. Their role encompasses the tethering of $\mathrm{K}^{+}$channels and AMPAR receptors to postsynaptic membrane, regulating the trafficking and function of AMPA and NMDA receptors in addition to sustaining a very complex yet highly organized molecular network.

The DLG-MAGUK protein family in mammals consists of four members, synapseassociated protein-90 (SAP-90)/postsynaptic density protein 95 (PSD-95) (Cho et al., 1992), chapsyn-110 (PSD-93) (Brenman et al., 1996), synapse-associated protein 102 (SAP102) (Müller et al., 1996) and synapse-associated protein 97 (SAP97) (Lue et al., 1994). All four of these proteins share a common domain structure. They consist of three PSD-95/Discs large/zona occludens-1 (PDZ) domains, one Src-homology 3 (SH3) domain and a catalytically inactive C-terminal guanylate kinase (GK) domain (Figure 1). PDZ domains are responsible for the major protein-protein interactions involving binding to voltage- and ligand-gated ion channels as well as cell adhesion molecules. On the other hand, SH3 and GK domains contribute to intra-molecular and intermolecular connections besides mediating non-PDZ protein interactions (McGee et al., 2001a; Montgomery et al., 2004). The interacting partners of SH3 and GK domains include microtubule associated protein (MAP1A), guanylate kinase domain-associated protein/synapse-associated protein-associated protein (GKAP/SAPAP) family which generates a link between the activity of ionotropic and metabotropic glutamatergic receptors and A kinase-anchoring protein 79/150 (AKAP79/150) important for NMDAR-dependent LTD in Schaffer collaterals (Montgomery et al., 2004; Xu et al., 2008; Bhattacharyya et al., 2009; Xu, 2011). In addition, SH3 and GK domains form a hook region which allows specific protein binding sites to be exposed according to the open and closed state (Masuko et al., 1999; Paarmann et al., 2002). 


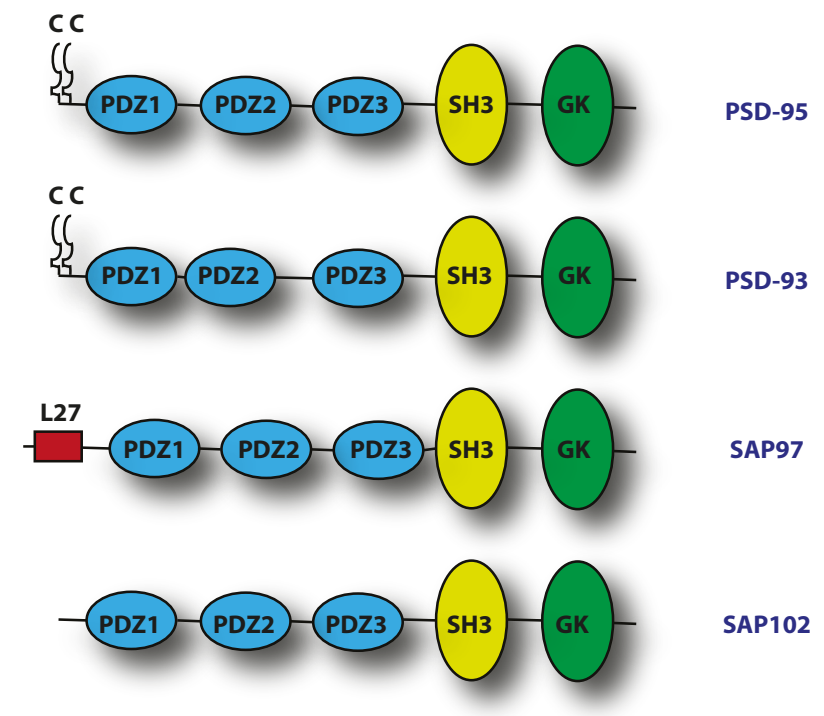

Figure 1: DLG-MAGUK family members.

DLG-MAGUKs consist of four members, which share a common domain structure with three PDZ, one SH3 and one GK domain. The N-termini of the proteins differ in an isoform-specific manner. Abundant forms of PSD-95 and PSD-93 contain two cysteine residues at the N-terminus, whereas SAP97 has a L27 and SAP102 a zing-finger motif.

Despite the fact that all DLG-MAGUKs share a common structure, the N-terminus of the proteins vary in a great extent hence giving them unique properties. The $\mathrm{N}$ terminus variation is also correlated with differences in the isoform function. Two isoforms of PSD-95 and SAP97 were introduced, functioning in an activity-dependent and independent manner. Alpha isoforms work in an activity-independent fashion and possess two cysteine residues at their $\mathrm{N}$-termini, which are accessible to palmitoylation (Schlüter et al., 2006). Particularly for PSD-95, the palmitoylation of these residues (C3 and C5) were indicated to be crucial for the protein function, regarding the N-terminal multimerization (Hsueh and Sheng, 1999), synaptic targeting and clustering (Topinka and Bredt, 1998; Craven et al., 1999; El-Husseini et al., 2000a). On the other hand, beta isoforms are involved in the activity-dependent events and contain an L27 domain (Lee et al., 2002b; Nakagawa et al., 2004; Schlüter et al., 2006). Even though both isoforms exist, PSD-95 is mainly expressed as the alpha isoform (Chetkovich et al., 2002) whereas SAP97 is found abundantly with its beta isoform in the synapse (Schlüter et al., 2006). Besides these two MAGUKs, PSD-93 has six isoforms (Parker et al., 2004). 
Two of the isoforms exhibit similarity to alpha isoform carrying two cysteine residues at the N-terminus (El-Husseini et al., 2000b). Another isoform resembles beta Nterminus with the L27 domain and the remaining three isoforms are unique to PSD-93. Lastly, SAP102 remains as a single isoform and hosts a zinc-finger motif at the Nterminus (El-Husseini et al., 2000b).

The expression profile of MAGUKs changes over the course of development similar to GluN2 subunits. SAP102 is highly expressed starting around P2 whereas the expression of PSD-95 and PSD-93 expression starts low directly after birth and increases over the first three weeks of the postnatal period. This increase is kept stable throughout the adulthood (Sans et al., 2000).

The mobility and the localization of the MAGUKs differ as well. For instance, PSD-95 is highly concentrated at the PSD of the synapses with a low mobility (only $36 \%$ being mobile at the spines), in contrary, SAP102 is highly mobile (80\%) and is found both in PSD and the cytoplasm of spines (Zheng et al., 2010, 2011).

These differences in the structure, expression profile, localization and mobility of the MAGUKs assigns them to specific functions regarding the basal synaptic transmission and synaptic plasticity.

\subsubsection{The role of DLG-MAGUKs in basal synaptic transmission and synaptic plasticity}

DLG-MAGUKs exert their effect on AMPAR and NMDAR function by interacting with the receptors in direct and indirect means. PSD-95, PSD-93 and SAP102 regulate the AMPA receptor transportation via their interaction with transmembrane AMPAR regulatory proteins (TARPs), which bind both MAGUKs and AMPA receptors. On the other hand, SAP97 is the only member, which can directly bind to GluA1 subunit of AMPARs (Leonard et al., 1998). Besides interacting with AMPA receptors, the members of this family were shown to bind the C-terminus of GluN2 subunits A and B through their PDZ domains (Kornau et al., 1995; Niethammer et al., 1996). The ability of MAGUKs to interact with both AMPA and NMDA receptors makes them suitable signal conveyers linking both receptor types thus regulating basal synaptic transmission and synaptic plasticity in excitatory synapses. 
The functional relevance of these proteins was revealed by the studies overexpressing or knocking-down each protein via RNA interference (RNAi) in different culture systems, as well as generating knockout mouse lines to examine the consequences of single or double MAGUK absence.

Overexpression studies of the dominant form of SAP97, SAP97 beta, showed inconclusive effects on the basal synaptic transmission. The results were either pointing to an enhancement of AMPAR (Rumbaugh et al., 2003; Nakagawa et al., 2004) and NMDAR transmission (Howard et al., 2010) or to no change in both components (Schnell et al., 2002; Ehrlich and Malinow, 2004; Schlüter et al., 2006). Germ-line deletion of SAP97 resulted in a lethal phenotype of the mouse line (Caruana and Bernstein, 2001), however conditional knockout mice demonstrated no deficit in basal synaptic transmission and long-term potentiation (Howard et al., 2010).

SAP102, on the other hand, slightly enhanced basal synaptic transmission when it was over-expressed (Schnell et al., 2002), but it did not cause any change when knocked-down (Elias and Nicoll, 2007). Interestingly, this knock-down effect was time-dependent, as only the manipulations in early development would result in a decrease in AMPA receptor responses but not in adulthood (Elias et al., 2008). Additionally, when tested in PSD-95/PSD-93 double knockout animals, SAP102 absence resulted in a 55\% reduction in AMPAR mediated responses (Elias and Nicoll, 2007). Knockout of SAP102 exhibited normal basal synaptic transmission, but showed an increase in high-frequency induced and spike-timing dependent LTP (Cuthbert et al., 2007).

The last two MAGUKs, PSD-93 and PSD-95, were considered similar with their roles in basal synaptic transmission and regulation of AMPA receptor function. Nevertheless, differences exist regarding their effect on synaptic plasticity (Carlisle et al., 2008). Overexpression of PSD-93 displays an enhancement of AMPA receptor responses. In consistency with this result, acute knock-down of PSD-93 results in 50\% reduction of AMPAR mediated transmission (Elias et al., 2006). Analysis of PSD-93 knockout mice revealed normal basal synaptic transmission (McGee et al., 2001b; Elias et al., 2006) and LTD but deficits in LTP (Carlisle et al., 2008). 
PSD-95, the classic scaffolding protein found in the PSD of excitatory synapses, is the last member of the DLG-MAGUK family deliberately investigated with its role in neurodegenerative diseases and neurodevelopmental disorders in addition to its role in synaptic processes such as synaptic maturation and synaptic transmission. Overexpression studies of PSD-95 beget an enhancement in the AMPAR transmission without a change in NMDAR responses (El-Husseini et al., 2000c; Schnell et al., 2002). It also resulted in an increase of miniature EPSC amplitude and frequency (Stein et al., 2003; Ehrlich and Malinow, 2004). Following this line, knock-down of PSD-95 caused a 50\% reduction in AMPAR mediated responses (Nakagawa et al., 2004; Elias et al., 2006; Schlüter et al., 2006). These effects on AMPA receptor responses were accompanied in some cases by minor changes in NMDAR EPSC amplitudes (Ehrlich et al., 2007; Futai et al., 2007; Kim et al., 2007). Efforts to dissect further the involvement of specific PSD-95 domains revealed that the expression of the first PDZ1-2 domains in conjunction with the intact $\mathrm{N}$-terminus was not enough to generate a functional PSD-95 protein. Moreover, this observation was dependent on the presence of endogenous PSD-95, in the presence of endogenous PSD-95, PDZ1-2 expression could enhance the AMPAR responses but knocking-down the endogenous form in combination with PDZ1-2 expression through the molecular replacement technique decreased the AMPA receptor transmission (Migaud et al., 1998; Schnell et al., 2002; Schlüter et al., 2006). A further study by $\mathrm{Xu}$ et al., 2008 demonstrated the requirement of SH3 and GK domains in basal synaptic transmission. However, the separate roles of SH3 and GK domains in this process are not fully understood. Knockout mouse lines of PSD-95 showed differences in basal synaptic transmission based on the strategy used to generate the knockout animals. Migaud et al. and Yao et al. observed no difference in basal synaptic transmission, where either a truncation of PSD-95 gene was used only leaving PDZ1 and 2 domains (Migaud et al., 1998) or a deletion of GK domain leaving no residual PSD-95 was introduced (Yao et al., 2004). In contrast to these results, a knockout line with a complete PSD-95 gene deletion showed reduced AMPAR responses (Béïque et al., 2006). Nevertheless, all of these knockout mice displayed enhanced LTP and an absence of LTD (Migaud et al., 1998; Béïque et al., 2006). This observation was supported by the acute knock-down of PSD-95 exhibiting enhanced 
LTP and absence of LTD (Ehrlich et al., 2007; Xu et al., 2008), and overexpression of PSD-95 exerting the opposite effect by occluding LTP and enhancing LTD (Béique and Andrade, 2003; Stein et al., 2003). In addition, the effect of PSD-95 knock-down on the basal synaptic transmission was going in line with the studies showing decreased AMPA/NMDA EPSC ratios (Béïque et al., 2006; Carlisle et al., 2008).

Despite the absence of basal synaptic transmission deficits observed in most of the single knockouts of DLG-MAGUKs (Migaud et al., 1998; McGee et al., 2001b; Cuthbert et al., 2007; Howard et al., 2010), if two or more of them are vanished from the neurons via knockout and/or knock-down techniques, bigger impacts on AMPAR mediated transmission is noticed. This implies a compensation mechanism among the MAGUK family members (Cuthbert et al., 2007), which is also supported by the increase in SAP102 levels in PSD-95 and PSD-93 double knockout animals (Elias et al., 2006) and by the compensation of PSD-95 loss through SAP97 beta expression (Schlüter et al., 2006).

The correlation of PSD-95 levels with the strength of AMPAR transmission and the fact that PSD-95 interacts with AMPA receptors through stargazin (one of the TARP members) triggered the hypothesis that PSD-95 should act like a 'slot' protein for AMPA receptors.

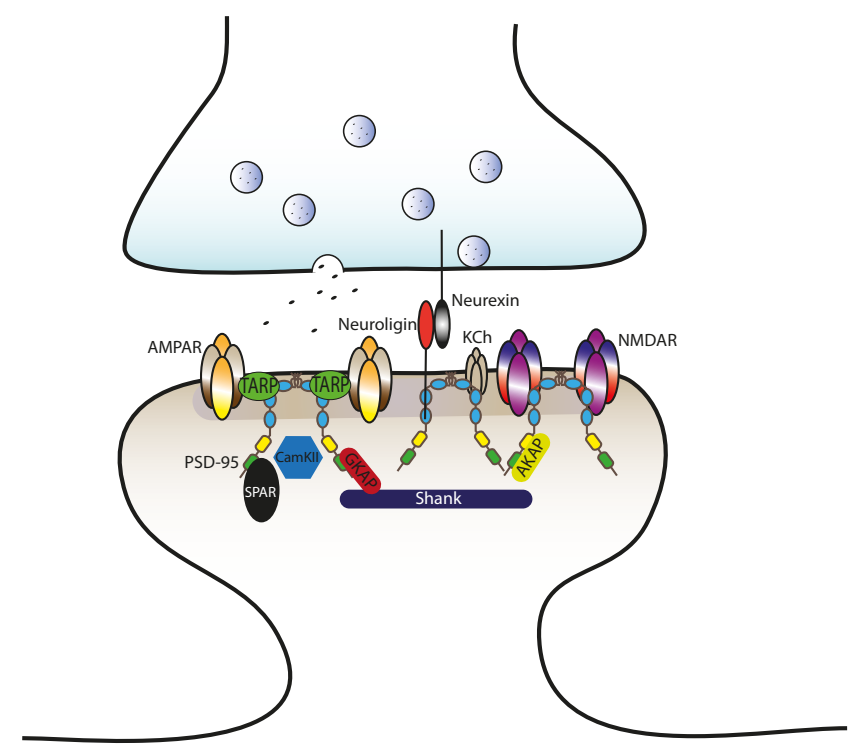


Figure 2: A minimized sketch of PSD-95 interactions with synaptic receptors, ion channels and signaling molecules in the postsynaptic density.

PSD-95 interacts with AMPA receptors through TARPs and binds to NMDA receptors directly. This allows it to work as a crucial signaling scaffold molecule, connecting synaptic receptor activations to down-stream molecules.

However, the absence of LTD and the enhancement of LTP in knockout animals counteract this hypothesis and rather suggest PSD-95 as a regulatory element of AMPAR trafficking at the synapse. In addition to its role in the regulation of AMPA receptor trafficking, PSD-95 binds to NMDA receptor C-terminus directly through its PDZ2 domain. This enables PSD-95 to act as a signal-conveyer between the NMDA receptors and down-stream signaling molecules (Figure 2). Owning such an important task, PSD-95 is engaged in several synaptic processes, starting from synapse development and maturation to the regulation of synaptic receptor function involving NMDARs.

\subsubsection{The role of PSD-95 in synaptic maturation and developmental NMDA receptor subunit switch}

The contribution of PSD-95 to synaptogenesis and synapse maturation was shown by the studies where PSD-95 expression and clustering in different brain regions was correlated with an increase in spine morphogenesis regarding the number and size of the spines (Okabe et al., 1999, 2001; El-Husseini et al., 2000c; Losi et al., 2003a). A similar maturation pattern related to PSD-95 expression was observed in the presynaptic terminals, most likely due to the fact that PSD-95 interacts with neuroligin on the postsynaptic side, which in turn binds to neurexin, a presynaptic protein, and exerts a retrograde effect (Scheiffele et al., 2000; Futai et al., 2007). Supporting this observation, the acute knock-down of PSD-95 prevents proper synapse formation and function. It also elicits smaller increase in the spine size after chemical LTP induction (Ehrlich et al., 2007). Knockout animals of PSD-95 present higher number of silent synapses, synapses containing NMDA but not AMPA receptors (Béïque et al., 2006). Lastly, PSD-95 clustering was emphasized in the stabilization of newly formed spines (Prange and Murphy, 2001).

One of the most important processes taking place during synaptic maturation is the developmental NMDA receptor subunit switch, which was mentioned in section 1.1.2.2. 
Until now several groups collected data pointing out the involvement of PSD-95 in this particular switch. The first strong evidence was raised by the detection of the changes in protein levels, regarding SAP102, PSD-95, GluN2B and GluN2A in rat hippocampus. The elevation in GluN2A level was corresponding with the one of PSD95 and the high expression level of GluN2B in early postnatal period corresponds to strong expression of SAP102 within this time frame (Sans et al., 2000). This observation suggests that the GluN2A level increase might be linked to the elevation in the PSD-95 expression levels (Figure 3). Indeed the co-immunoprecipitation experiments demonstrated a preference in the binding of PSD-95 to GluN2A and a preference of SAP102 binding to GluN2B (Sans et al., 2000). Following these results, in PSD-95 knockout animals stronger GluN2B mediated currents were observed (Béïque et al., 2006). In addition, NMDA receptor subunit switch was absent in PSD95 and PSD-93 double knockout animals. In the same study, it is proposed that PSD-95 takes over the role from SAP102 to regulate the AMPAR and NMDAR trafficking in the later stages of development (Elias et al., 2008). Yet, another interesting point in this respect is the similarity of SAP102 to GluN2B and of PSD-95 to GluN2A regarding their localization and mobility. PSD-95 and GluN2A are located mostly at the synaptic site with a low turn-over rate, whereas SAP102 and GluN2B are detected also at the extra-synaptic site with higher mobility (Groc et al., 2006b; Zheng et al., 2010, 2011).

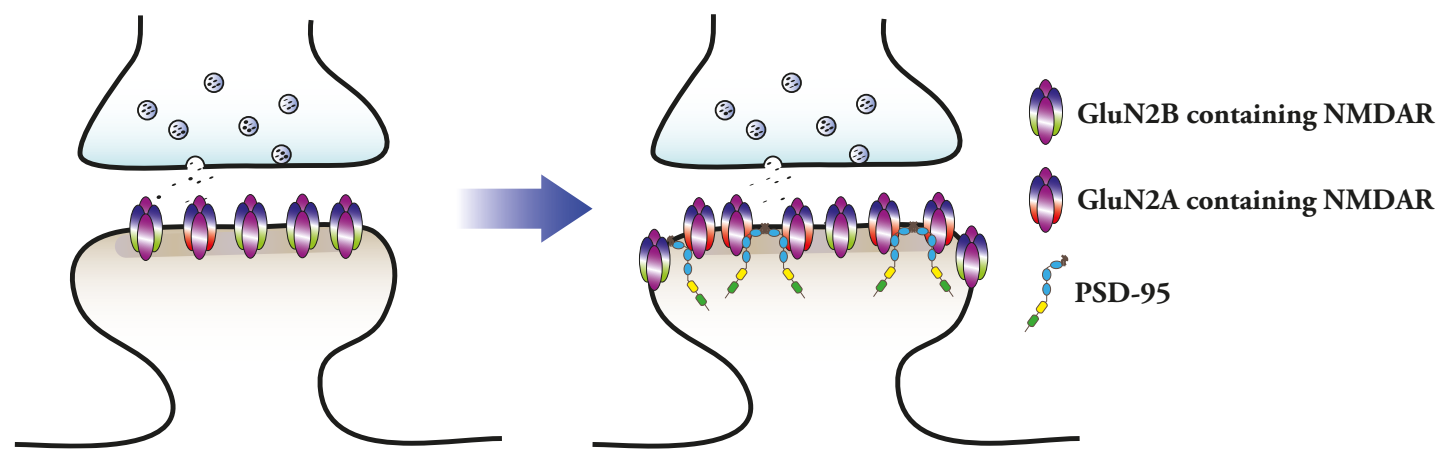

Figure 3: Developmental NMDA receptor subunit switch observed in synapses.

During early development NMDA receptors mostly consist of GluN2B-containing receptors. Upon activation a switch is triggered in NMDA receptor subunit composition from GluN2B-containing to GluN2A-containing receptors. This switch is accompanied by an increase in PSD-95 levels. 
Albeit some other studies in hippocampus could not observe a striking difference in the association of MAGUKs with GluN2 subunits (Al-Hallaq et al., 2007) and in the levels of GluN2A and GluN2B in the PSD-95 knockout mouse (Migaud et al., 1998), data from other brain regions provide means to believe that PSD-95, indeed, mediates such a switch. In cerebellar neurons, PSD-95 expression decreased the ifenprodil sensitivity of NMDAR responses and PSD-95 showed higher co-localization with GluN2A subunits in comparison to GluN2B (Losi et al., 2003a). In addition, in cortical and thalamic neurons, a GluN2B to GluN2A switch was accompanied by an increase in PSD-95 expression (Liu et al., 2004b).

Finally, another line of evidence assisting this phenomenon was obtained from visual cortex. Townsend et al. proposed an activity-dependent NMDA receptor subunit switch, which involves the targeting of PSD-95 to the synapse (Townsend et al., 2003). Moreover, Yoshii et al., 2003 showed an activity-dependent redistribution of PSD-95 in visual cortex neurons upon eye opening and an enhanced interaction between PSD-95 and GluN2A (Yoshii et al., 2003). Visual cortical pyramidal neurons of dark-reared animals exhibited higher number of silent synapses (Funahashi et al., 2013), which might be due to aberrant distribution of PSD-95. Furthermore, the synaptic distribution of PSD-95 could be manipulated via opening the eyes of the animal at a premature age or suturing and reopening the eye at a later stage (Yoshii et al., 2003). The possibility of controlling the activity-dependent subunit switch in visual cortex through eye opening makes it a special model to study the subunit switch concept.

Attempts to understand the mechanism underlying the NMDA receptor subunit switch yielded few data exposing the participation of group 1 metabotropic receptors and CKII kinase in this process. A knockout mouse line of mGluR5 exhibited deficits in the GluN2B to GluN2A switch both in CA1 region of hippocampus and the visual cortex. This pathway involved the activation of phospholipase C (PLC) and PKC (Matta et al., 2011). Another member of group 1 metabotropic receptor's, mGluR1's, dysfunction prevented the switch from taking place in ventral tegmental area (Bellone et al., 2011). Lastly, CKII activity was shown to promote the GluN2A-containing receptor presence in the synapse by stimulating the endocytosis of GluN2B-containing receptors (Sanz-Clemente et al., 2010). Interestingly, the endocytosis action is triggered 
by the phosphorylation of S1480 residue by CKII, which is located in the PDZ binding domain of GluN2B. The phosphorylation consequently disrupts the interaction between GluN2B and PSD-95 (Chung et al., 2004), which gives PSD-95 a potential role in the regulatory mechanism of NMDA receptor subunit switch. However, CKII is not the only kinase mediating PSD-95 and GluN2 subunit interaction thus controlling GluN2 subunit surface expression.

PSD-95 and kinase interactions were shown to be involved in the phosphorylation of PSD-95 itself as well as other proteins by using PSD-95 as the mediator (Gardoni et al., 2009). Some of the kinases directly phosphorylating PSD-95 include CamKII, c-Jun NH2-terminal kinase (JNK) and cyclin-dependent kinase 5 (CDK5). CamKII phosphorylates S73 within the PDZ1 domain of PSD-95, which in turn regulates the interaction of PSD-95 and CamKII with GluN2A subunit (Gardoni et al., 2006). JNK, on the other hand, phosphorylates S295 on PSD-95 and the phosphorylation of this residue promotes the PSD-95 clustering at the synapse and increases the amplitude of AMPAR EPSCs (Kim et al., 2007). CDK5, in addition, phosphorylates three aminoacids (T19, S25 and S35) at the N-terminus of PSD-95. Elimination of the CDK5 activity, hence the dephosphorylation of these sites, results in larger clusters of PSD-95 at the synapse, potentially regulating ion channel clustering (Morabito et al., 2004). Regarding the kinase interactions where PSD-95 is the mediator, the most prominent example is the interaction of Src kinase family with PSD-95 to modulate the phosphorylation state of NMDA receptors, particularly GluN2B. Src kinase binds to the N-terminus of PSD-95 via its SH2 domain (Kalia et al., 2006). Binding of Src kinases to PSD-95 allows the kinase interaction with GluN2B subunit, phosphorylating Y1472 site and blocking AP2-clathrin mediated endocytosis of GluN2B-containing NMDA receptors (Prybylowski et al., 2005). Moreover, the ability of Src kinases to interact with MAGUK family members was shown to be restricted to PSD-95 (Kalia and Salter, 2003).

Interestingly, a recent study by Zhang et al., 2008 proposed a mechanism where the interaction between PSD-95, Src kinase and GluN2B is regulated according to the phosphorylation state of PSD-95 by CDK5. Once PSD-95 is phosphorylated at CDK5 phosphorylation sites, its interaction with Src kinase is interrupted. This interruption 
causes dephosphorylation of GluN2B Y1472 site and eventually triggers the endocytosis of GluN2B-containing NMDA receptors through the AP2-clathrin mediated pathway (Zhang et al., 2008).

Such interplay between PSD-95, Src kinase and GluN2B opens the possibility to understand more about the developmental NMDA receptor subunit switch, which might be CDK5 activity-dependent.

\subsection{Scope of the thesis}

PSD-95, an important scaffold protein in the postsynaptic density, is known to regulate the AMPA receptor function at the excitatory synapses. PSD-95 was shown to be phosphorylated by CDK5 at its N-terminus, however the functional consequences of this phosphorylation are not known yet. Moreover, it is not clear how the specific domains of PSD-95 contribute to the regulation of basal synaptic transmission.

In this project by using dual whole cell patch-clamp technique in combination with the molecular replacement strategy, in vivo \& in vitro viral injections and biochemical assays I aimed to understand the following points:

- The CDK5 phosphorylation effect on PSD-95 with respect to the basal synaptic transmission in CA1 region neurons of rat hippocampus.

- The CDK5 phosphorylation effect on PSD-95 with respect to the developmental NMDA receptor subunit switch in layer $2 / 3$ neurons of mouse visual cortex.

- The specific functions of single PSD-95 domains with respect to the basal synaptic transmission in CA1 region neurons of rat and PSD-95 knockout mouse hippocampus.

Addressing these concepts will create a better understanding about the mechanisms regulating the basal synaptic transmission and will help us to understand how learning and memory is governed in the brain. 


\section{Materials \& Methods}

\subsection{Molecular Biology}

\subsubsection{Cloning}

Standard methods were used for the cloning procedure (Sambrook, 2001). The chemicals and kits used for the cloning steps were obtained from Bioline (Luckenwalde, Germany), Fermentas/Thermofischer Scientific (Waltham, MA, USA), NEB (Frankfurt, Germany) and Qiagen (Hilden, Germany).

The generation of the DNA constructs involved the use of the following primers produced by Sigma- Genosys (Steinheim, Germany) or MPI-EM AGCT Sequencing Facility (Göttingen, Germany) (Table 1). 
Table 1: The list of forward and reverse primers used for the cloning and sequencing of the constructs.

\section{Primer list}

\begin{tabular}{|c|c|}
\hline \multicolumn{2}{|l|}{ Cloning } \\
\hline PacI fwd & GGGTTTATTACAGGGACAGC \\
\hline P95 19A rev & TCGTCTCAAGAGGAGGCGCGTCTTCATCTTGGTAGCGG \\
\hline P95 19D rev & TCGTCTCAAGAGGAGGATCGTCTTCATCTTGGTAGCGG \\
\hline P95 S35A fwd & AGCACACCTACCTAATCAGGCCAATGCTCCCCCTGTGA \\
\hline \multirow{3}{*}{ P95 S35D fwd } & TTGTCAACAC \\
\hline & AGCACACCTACCTAATCAGGCCAATGATCCCCCTGTGA \\
\hline & TTGTCAACAC \\
\hline P95 Nde rev ERI & GCGAATTCGGTACACAACGTCATATGTGTTC \\
\hline P95 25A fwd & TCGTCTCACTCTGGAACACGCACCAGCACACCTACCTA \\
\hline \multirow{3}{*}{ P95 25D fwd } & ATCAGG \\
\hline & TCGTCTCACTCTGGAACACGATCCAGCACACCTACCTA \\
\hline & ATCAGG \\
\hline rP95 fwd XbaI(16682) & TCGTCTAGACCACCATGGACTGTCTCTGTATAGTG \\
\hline IRES fwd AgeI & CGACCGGTCCCCTCTCCCTCCCC \\
\hline P95 rev AgeI & CGACCGGTTCAGAGTCTCTCTCGGGCTGG \\
\hline EGFP rev (14952) & ACCCCGGTGAACAGCTCC \\
\hline DNK5 fwd XbaI & CGCTCTAGACCACCATGCAGAAATACGAGAAACTGG \\
\hline DNK5 rev BamHI & CGGGATCCTTAAFFCGGACAGAAGTCGGAG \\
\hline P25 fwd XbaI & АTCTCTAGACCACCATGGCCCAGCCCC \\
\hline P35 fwd XbaI & АTCTCTAGACCACCATGGGCACGGTGCTG \\
\hline P35 rev BamHI & CGGGATCCCACCGATCCAGGCCTAG \\
\hline \multicolumn{2}{|l|}{ Sequencing } \\
\hline PacI fwd & GGGTTTATTACAGGGACAGC \\
\hline EGFP rev & ACCCCGGTGAACAGCTCC \\
\hline pUb fwd & GTCCGCTAAATTCTGGCCGTT \\
\hline WPRE rev 2 & GCAGCGTATCCACATAGCGT \\
\hline
\end{tabular}




\subsubsection{Polymerase chain reaction (PCR)}

For the amplification of specific DNA fragments via PCR (Saiki et al., 1985), the indicated amounts of the following contents were used (Table 2).

Table 2: Reaction mix for polymerase chain reaction. PCR Mix

Template DNA $20 \mathrm{ng}$

10x Reaction Buffer (Bioline/Fermentas) $\quad 2 \mu 1$

dNTP's (2.5 mM each, Bioline) $\quad 2 \mu \mathrm{l}$

$50 \mu \mathrm{M}$ Forward primer $\quad 0.5 \mu \mathrm{l}$

$50 \mu \mathrm{M}$ Reverse primer $\quad 0.5 \mu \mathrm{l}$

Taq*/ Pfu** Polymerase (Bioline/Fermentas) $1 \mu 1$

$20 \mu 1$

* (Saiki et al., 1988)

** (Lundberg et al., 1991)

PCR products were run on $1 \%$ agarose gel prepared with TAE Buffer $(0.2 \% 0.5 \mathrm{M}$ EDTA, $1.14 \mathrm{ml} / 1$ Acetic acid, $30 \mathrm{mM}$ Tris) in order to purify and confirm the amplification of PCR products.

\subsubsection{Restriction digestion}

For digestion reactions, restriction enzymes from Fermentas and NEB were used with their respective buffers. The typical digestion reaction protocol was as shown in Table 3. 
Table 3: Reaction mix for restriction digestion.

Restriction Digestion Reaction Mix

\begin{tabular}{ll} 
Sample & $1 \mu \mathrm{l}(1.5 \mu \mathrm{g})$ \\
$10 \mathrm{x}$ Enzyme Buffer & $5 \mu \mathrm{l}$ \\
Restriction Enzyme & $2 \mu \mathrm{l}$ \\
$\mathrm{H}_{2} \mathrm{O}$ & $42 \mu \mathrm{l}$ \\
& \\
\cline { 2 - 2 } & $50 \mu \mathrm{l}$
\end{tabular}

The reaction was kept for 2 hours at $37^{\circ} \mathrm{C}$ unless a different temperature was specified for the selected enzyme. The digested sample was run on $1 \%$ agarose gel prepared with TAE buffer. The expected band was excised from the gel and purified according to the protocol indicated in section 2.1.1.3.

\subsubsection{PCR product and agarose gel purification}

The PCR purification and restriction digest products were isolated according to the manufacturer's protocol of Qiagen. The isolated fragments were collected in $20-30 \mu 1$ of 1:10 diluted elution buffer (Qiagen).

\subsubsection{Ligation}

The inserts were ligated into different vector backbones with a 1:3 vector to insert molar ratio. For a total volume of $30 \mu 1$ ligation mix, $3 \mu \mathrm{l}$ of T4 DNA Ligase Buffer (Fermentas) and $1 \mu \mathrm{l}$ of T4 DNA Ligase (Fermentas) were used. The ligation reaction was kept at $16^{\circ} \mathrm{C}$ overnight. 


\subsubsection{Transformation}

The ligation product was introduced into XL1-Blue Escherichia coli (E. coli) strain (Stratagene, Santa Clara, CA, USA) and colonies were grown on ampicillin-containing LB agar plates $(100 \mu \mathrm{g} / \mathrm{ml}$, Roth).

\subsubsection{Plasmid preparation}

Single colonies were picked from the transformed bacteria plates. The colonies were inoculated either in $5 \mathrm{ml} \mathrm{LB}$ medium for mini-prep or $50 \mathrm{ml} \mathrm{LB}$ medium for maxi-prep. In both cases, ampicillin $(100 \mu \mathrm{g} / \mathrm{ml})$ was added to the media to promote selective bacteria growth. The bacteria were allowed to grow overnight at $37^{\circ} \mathrm{C}$ under vigorous shaking.

The bacteria were collected via centrifugation and the DNA was isolated according to Qiagen (Hilden, Germany) DNA isolation protocol following alkaline lysis (Birnboim and Doly, 1979). In order to eliminate protein contamination, phenolchloroform extraction was applied. First the DNA solution first was mixed with equal volume of phenol:chloroform:isoamyl alcohol (25:24:1) (Applichem) and centrifuged. Then the obtained aqueous phase was isolated and mixed with equal volume of chloroform. After the purification, DNA was precipitated with 0.7 volumes of isopropanol and resuspended in $30 \mu$ l elution buffer (Qiagen) for mini prep and $200-$ $300 \mu 1$ for maxi-prep.

Sequencing of the generated constructs was performed at the AGCT Sequencing Facility of MPI-EM (Göttingen).

\subsubsection{Expression constructs}

For the generation of all lentiviral vector based constructs, FUGW (Lois et al., 2002), FRUGW containing RNAi against endogenous PSD-95 (provided by Dr. Dr. Oliver Schlüter) or bicistronic lentiviral vector FUp93alviGW (provided by Dr. Juliane Krüger) were used as backbone. The production of FRUGW construct involved the swopping of the RNAi sequence in FHUGW (Schlüter et al., 2006) with 'TCACGATCATCGCTCAGTATA' sequence (Elias et al., 2006) to achieve higher 
knock-down efficiency both in rat and mouse cultures. The scheme of the vector backbones used is shown in Figure 4.
A
Ubq. P EGFP WPRE

B

H1 p. ShRNA Ubq.P EGFP WPRE

Figure 4: Lentiviral vectors used for cloning.

(A) FUGW vector with the EGFP expression under the Ubiquitin promoter. The transcription is enhanced through the WPRE cassette downstream of EGFP coding sequence.

(B) FRUGW vector with $\mathrm{H} 1$ promoter enabling the shRNA expression targeting endogenous PSD95 and Ubiquitin promoter expressing EGFP and the protein of interest. The transcription is enhanced through the WPRE cassette downstream of EGFP coding sequence.

The constructs generated throughout this study are listed in Table 4. 
Table 4: List of the DNA constructs generated during the study.

Expression constructs

FRUr95A3GW

$(\operatorname{sh} 95+$ p95A3::GFP)

FRUr95D3GW

$(\operatorname{sh} 95+$ p95D3::GFP)

FRUr95G

(sh95 + p95::GFP without WPRE)

FRUr95A3G

$(\operatorname{sh} 95+$ p95A3::GFP without WPRE)

FRUr95D3G

$(\operatorname{sh} 95+$ p95D3::GFP without WPRE)

FRUr95viG

$(\operatorname{sh} 95+$ p95viGFP $)$

FRUr95A3viG

$(\operatorname{sh} 95+$ p95A3viGFP $)$

FRUr95D3viG

$(\operatorname{sh} 95+$ p93D3viGFP $)$

FUDNK5viGW

(DNK5viGFP)

FUp25viGW

(p25viGFP)
Triple alanine substituted PSD-95 with

GFP fusion and WPRE cassette

Triple aspartate substituted PSD-95 with

GFP fusion and WPRE cassette

Wild-type PSD-95 with GFP fusion and without WPRE cassette

Triple alanine substituted PSD-95 with

GFP fusion and without WPRE cassette

Triple aspartate substituted PSD-95 with

GFP fusion and without WPRE cassette

Wild-type PSD-95 with separate GFP

expression via IRES cassette, no WPRE

cassette

Triple alanine substituted PSD-95 with separate GFP expression via IRES

cassette, no WPRE cassette

Triple alanine substituted PSD-95 with

separate GFP expression via IRES

cassette, no WPRE cassette

Overexpression of dominant-negative

CDK5 with separate GFP expression via

IRES cassette

Overexpression of CDK5 co-activator p25 with separate GFP expression via 
IRES cassette

FUp35viGW

(p35viGFP)
Overexpression of CDK5 co-activator p35 with separate GFP expression via

IRES cassette

\subsubsection{Generation of CDK5 mutants of PSD-95 as GFP fusion} protein under the transcription enhancer WPRE cassette in lentiviral vector

To generate mutants of PSD-95 mimicking two different states of CDK5 phosphorylation, three aminoacid residues at the PSD-95 N-terminus, T19, S25 and S35 were substituted either with alanine or aspartate aminoacids, mimicking phosphorylated or non-phosphorylatable states respectively. The N-terminal of PSD-95 sequence was amplified from FUp95GW template (PSD-95 overexpression vector provided by Dr. Dr. Schlüter) by using PacI fwd and p95 19A/ p95 19D rev primers. The product was cut with SpeI and Esp3I, and a100 bp band was isolated. A second nested PCR was done on FUp95GW with the primers p95 S35A/95 S35D fwd and p95 Nde rev ERI. The 700 bp PCR product was then amplified with p95 25A/S25D fwd and p95 Nde rev ERI primers. The final product was cut with EcoRI and Esp3I. Both 100 bp and 700 bp fragments were subcloned into pBluescriptII SK(-) (pBSKII) (Stratagene, Santa Clara, CA, USA) cut with SpeI and EcoRI. pBSKII carrying the mutant PSD-95 forms were digested with $\mathrm{XbaI}$ and NdeI, and a $700 \mathrm{bp}$ band was isolated. FRUr95GW vector (wild-type PSD-95 replacement construct provided by Dr. Dr. Schlüter) was cut with NdeI and BsrGI, and a $2.2 \mathrm{~kb}$ band was isolated. Both $700 \mathrm{bp}$ and $2.2 \mathrm{~kb}$ bands were cloned into FRUr95GW digested with $\mathrm{XbaI}$ and BsrGI. The constructs were called FRUr95A3GW for non-phosphorylatable mutant and FRUr95D3GW for phosphor-mimicking mutant. 


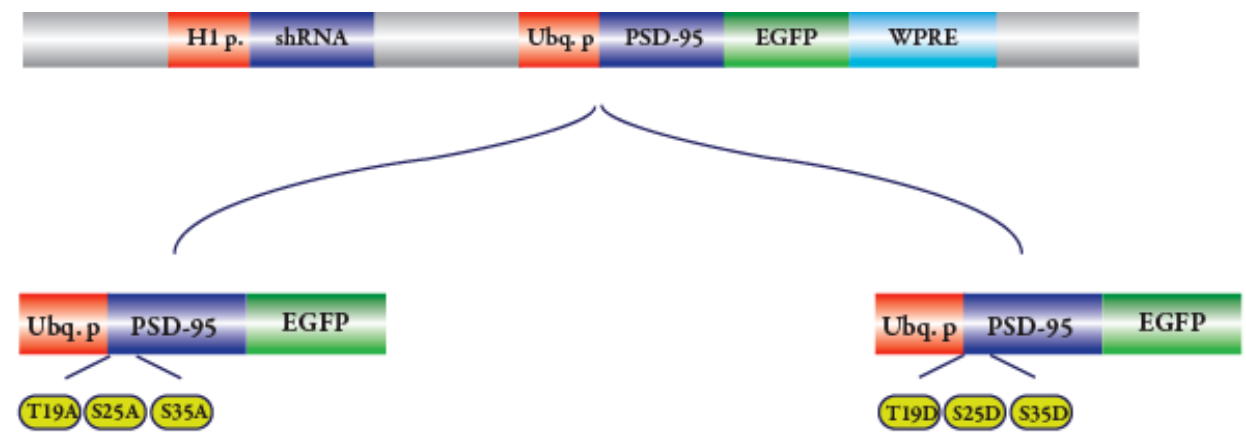

Non-phosphorylatable mutant of PSD-95

Phospho-mimicking mutant of PSD-95

Figure 5: CDK5 mutant constructs of PSD-95 with GFP-tag and enhanced expression.

Endogenous PSD-95 was knocked-down by the expression of shRNA sequence under the H1 promoter. The expression of PSD-95 with GFP-tag was carried on under the Ubiquitin promoter and enhanced by the down-stream WPRE cassette. T19, S25 and S35 aminoacids at the N-terminus of PSD-95 were substituted with either alanine or aspartate aminoacids to mimick non-phosphorylatable or continously phosphorylated states respectively.

\subsubsection{Generation of CDK5 mutants of PSD-95 as GFP fusion}

\section{protein without the WPRE enhancer cassette in lentiviral vector}

Wild-type replacement construct of PSD-95 as well as the replacement with CDK5 mutants of PSD-95 were cloned without the WPRE cassette in order to have lower expression levels to match the endogenous state. WPRE cassette was cut out from the FRUr95GW (provided by Dr. Dr. Schlüter), FRUr95A3GW and FRUr95D3GW constructs with ClaI enzyme and the rest of the vector was ligated again (Figure 6). The constructs were named as FRUr95G, FRUr95A3G and FRUr95D3G.

\section{H1 p. ShRNA Ubq.P PSD-95 EGFP}

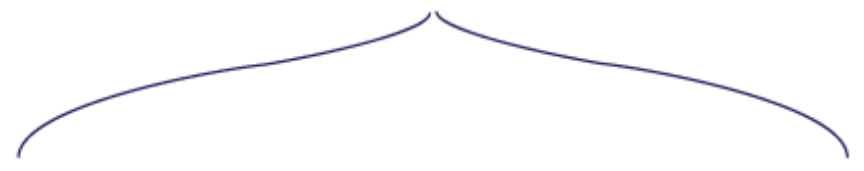

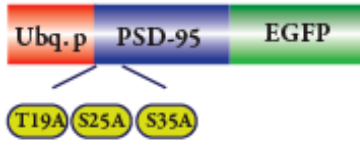

Non-phosphorylatable mutant of PSD-95

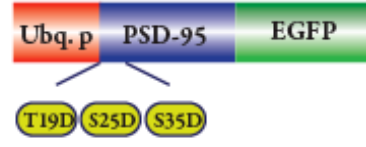

Phospho-mimicking mutant of PSD-95

Figure 6: CDK5 mutant constructs of PSD-95 with GFP-tag and without the enhancer effect. The expression of PSD-95 shRNA was preserved under the H1 promoter and the mutant PSD-95 proteins were expressed following the Ubiquitin promoter. Transcription was not enhanced through the WPRE cassette. 


\subsubsection{Generation of CDK5 mutants of PSD-95 in bicistronic lentiviral vector with separate GFP expression via IRES cassette}

The PSD-95 mutant constructs showed a very strong expression level, potentially masking the mutation effect. In addition, the GFP fusion interfered with the interaction of PSD-95 with its binding partners. Therefore, the mutants were also cloned in the bicistronic lentiviral backbone that included the separate translation of GFP via IRES (internal ribosome entry side) sequence and lacked the WPRE transcription enhancer cassette (Figure 7). The bicistronic vector backbone (FUp93a1viGW) was provided by Dr. Juliane Krüger (former Molecular Neurobiology lab member, ENI, Göttingen, Germany)(Krüger, 2010a). PSD-95 sequences were amplified from FRUr95GW, FRUr95A3GW and FRUr95D3GW vectors using rP95 fwd XbaI and P95 rev AgeI primers, and then were digested with XbaI. The digest products were subcloned into pBluescriptII SK(-) (pBSKII) (Stratagene, Santa Clara, CA, USA) cut with XbaI and SmaI . The PSD-95 sequence was cut out from pBSKII by XbaI and AgeI enzymes. IRES-GFP sequence was amplified from FUp93a1viGW vector with IRES fwd AgeI and EGFP rev primers and was subcloned into pBSKII cut with SmaI. IRES-GFP sequence was cut out from pBSKII by AgeI and BsrgI enzymes. FRUr95GW vector was cut with XbaI and BsrGI restriction enzymes and a triple ligation was set with vector backbone, PSD-95 sequences and IRES-GFP sequence. The constructs were named as FRUr95viG (wild-type replacement), FRUr95A3viG (non-phosphorylatable mutant replacement) and FRUr95DviG (phospho-mimicking mutant replacement).This approach enabled the endogenous expression levels of the mutants and prevented any potential interference with protein-protein interactions through GFP-tag. 


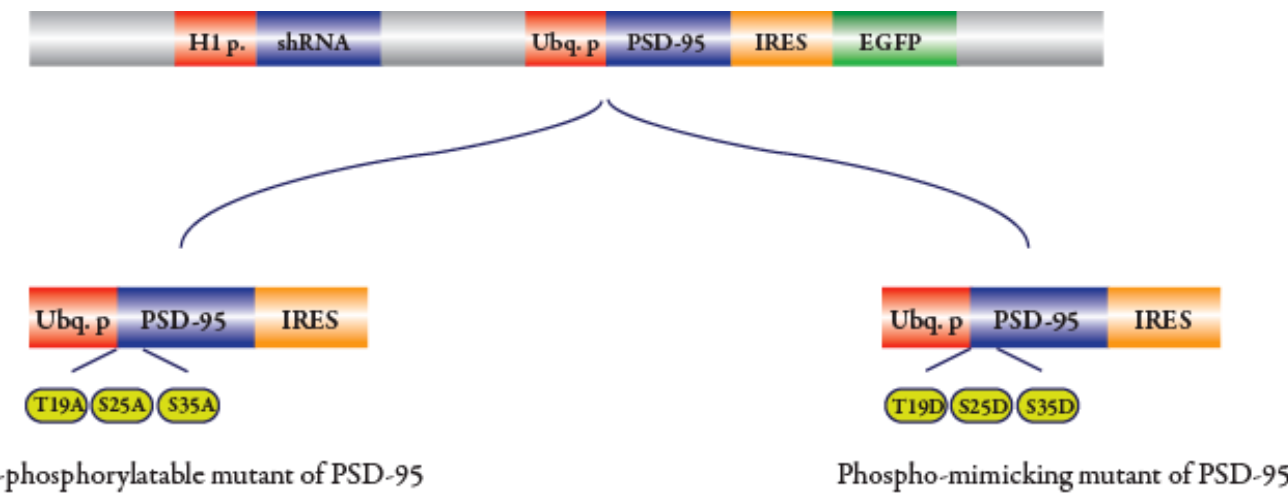

Non-phosphorylatable mutant of PSD-95

Phospho-mimicking mutant of PSD-95

Figure 7: CDK5 mutant constructs of PSD-95 with non-tagged GFP and endogenous levels of expression.

Endogenous PSD-95 was knocked-down by the expression of shRNA sequence under the H1 promoter. GFP was expressed separately from PSD-95 through the insertion of an IRES cassette between PSD-95 and EGFP coding sequences. The transcription enhancer WPRE was not included in order to establish endogenous levels of expression. T19, S25 and S35 aminoacids at the N-terminus of PSD-95 were substituted with either alanine or aspartate aminoacids to mimic non-phosphorylatable or continuously phosphorylated states respectively.

\subsubsection{Generation of dominant-negative CDK5, p25 and p35 overexpression constructs in lentiviral vector}

Overexpression constructs of dominant-negative form of CDK5 (DNK5), p25 and p35 were generated by cloning the sequences (kindly provided by Prof. Dr. André Fischer) into bicistronic lentiviral vector (FUp93alviGW) (Krüger, 2010b). For the amplification of DNK5 sequence DNK5 fwd XbaI and DNK5 rev BamHI primers were used, and the PCR product was inserted into the $\mathrm{XbaI}$ and $\mathrm{BamHI}$ restricton sites of the lentiviral backbone Sequences of CDK5 co-activators (p25 and p35) were amplified with p25 fwd XbaI and p35 fwd XbaI forward primers and p35 rev BamHI reverse primer. The sequences were inserted into the $\mathrm{XbaI}$ and $\mathrm{BamHI}$ restriction sites of the vector backbone. The constructs were names as FUDNK5viGW, FUp25viGW and FUp35viGW. 


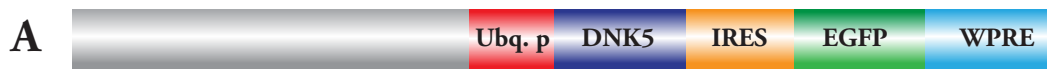

B

Ubq. P p25 IRES EGFP WPRE

C

$\begin{array}{llll}\text { Ubq. P } & \text { P35 IRES EGFP WPRE }\end{array}$

Figure 8: Overexpression constructs of dominant-negative CDK5 (DNK5), p25 and p35 in bicistronic lentiviral backbone.

(A) DNK5 expression was established under the Ubiquitin promoter. GFP expression was separated via IRES sequence and the construct expression was enhanced by WPRE cassette.

(B) P25 expression was established under the Ubiquitin promoter. GFP expression was separated via IRES sequence and the construct expression was enhanced by WPRE cassette.

(C) P35 expression was established under the Ubiquitin promoter. GFP expression was separated via IRES sequence and the construct expression was enhanced by WPRE cassette.

\subsubsection{PSD-95 domain mutants in lentiviral vector}

Mutants of PSD-95 lacking one or more domains, as well as the combination of the mutants with PSD-95, SAP102 shRNA's were generated by Dr. Stéphanie Bonnet (Bonnet, 2011). In addition, sh102 + p95 PDZ3::GFP and sh97 + p95 $\mathrm{GK}:$ :GFP construct were generated by swopping the shRNA sequence of PSD-95 with shRNA sequence of SAP102 or SAP97 (Li et al., 2011). The constructs sh95 + PDZ1/2SH3::GFP and sh102 + PDZ1/2-SH3::GFP were provided by Dr. Dr. Oliver Schlüter.

\subsection{Cell \& slice Culture}

The chemicals and the cell culture material were obtained from Biochrom (Berlin, Germany), Sigma Aldrich (München, Germany), Millipore (Schwalbach, Germany), Roth (Karlsruhe, Germany), Merck (Darmstadt, Germany), Greiner Bio-one (Frickenhausen, Germany), Invitrogen/Life Technologies (Darmstadt, Germany), Thermofischer Scientific (Waltham, MA, USA) and Roche (Mannheim, Germany). 


\subsubsection{HEK293T cell line}

Human embryonic kidney, HEK293T, cell line was used for the purposes of small and large scale virus preparation (Graham et al., 1977) as well as coimmunoprecipitation assays. The cells were cultured on $10 \mathrm{~cm}$ cell culture dishes in the presence of High glucose Dulbeccos's Modified Eagle's Medium (Biochrom) containing 10\% fetal calf serum (Biochrom). The cultures were kept in $37^{\circ} \mathrm{C}$ incubator with an equilibrated $5 \%$ $\mathrm{CO}_{2}$ concentration. Every 2-3 days, when the cells reached $75-80 \%$ confluency, a passage of a dilution of 1:12-20 was performed (Masters and Stacey, 2007).

\subsubsection{HEK293T cell transfection}

Transfection of the HEK293T cells was achieved by the means of polyethylenimine transfection method. $3 \mu \mathrm{g}$ of DNA was used to transfect a single well of a 6-well plate. The DNA was mixed with $100 \mu 1$ of $150 \mathrm{mM} \mathrm{NaCl}$ solution. After 10 minutes, $14 \mu 1$ of $15 \mathrm{mM}$ linear polyethylinimine solution was added to $100 \mu 1$ of $150 \mathrm{mM} \mathrm{NaCl}$ in a separate tube and the mixtures were incubated for 10 minutes. Finally, both of the tube contents were mixed and the transfection solution was added onto freshly split HEK293T cells.

Transfected cells were collected after 1.5 - 2 days of transfection for the purposes described in the following sections.

\subsubsection{Lentivirus production}

Lentiviral particles (Lewis et al., 1992) were produced by transfecting HEK293T cells with the vector of interest to be expressed, HIV-1 packaging vector $\Delta 8.9$ and envelope glycoprotein vector vsvg. For small scale virus production, 6 well cell culture plates and for large scale virus production T-75 cell culture flasks were used. The amounts of DNA used for each viral production are listed below (Table 5). 
Table 5: DNA construct and amounts used for small and large scale virus production.

$$
\text { Small scale virus production Large Scale virus }
$$

production

$\begin{array}{llc}\text { Vector of interest } & 2 \mu \mathrm{g} & 10 \mu \mathrm{g} \\ \Delta 8.9 \text { vector } & 1.5 \mu \mathrm{g} & 7.5 \mu \mathrm{g} \\ \text { Vsvg vector } & 0.5 \mu \mathrm{g} & 2.5 \mu \mathrm{g}\end{array}$

One day after transfection, the cultures were transferred to $32^{\circ} \mathrm{C}$ incubator with $5 \%$ equilibrated $\mathrm{CO}_{2}$ concentration in order to enhance viral production.

For the production of small scale virus, the supernatant was harvested after 2 days of transfection and centrifuged at $800 \mathrm{~g}$ for 5 minutes, in order to isolate viral particles from the rest of cell debris. The supernatant was aliquoted and stored at $-80^{\circ} \mathrm{C}$ for further use.

For the production of large scale virus, the supernatant from T-75 flask was collected and centrifuged at $2.000 \mathrm{~g}$ for 5 minutes. The solution was then run through the PVDF membrane with $0.45 \mu \mathrm{m}$ pore size (Millipore) and the viral particles were precipitated via ultracentrifugation at $36.500 \mathrm{~g}$ for 1.5 hours. The pellet was dissolved in virus storage buffer $(20 \mathrm{mM}$ Tris- $\mathrm{HCl}(\mathrm{pH}=8.0), 250 \mathrm{mM} \mathrm{NaCl}, 5 \%$ Sorbitol). The aliquots were kept at $-80^{\circ} \mathrm{C}$ for further use.

\subsubsection{Dissociated hippocampal neuron culture}

Dissociated hippocampal neuron cultures were prepared from P0 mouse or rat hippocampi (Huettner and Baughman, 1986). The animals were decapitated and the brain was gently placed in the dissection solution. The hemispheres were separated with two micro-dissecting tweezers. The meninges were removed and the portions corresponding to the ending and the beginning of hippocampus were cut off with a sharp spatula. The hippocampus was folded out from the cortex and detached. The dentate gyrus was removed from the rest of the hippocampus. All hippocampi were collected in Enzymatic Solution (ES: $11.39 \mathrm{mM}$ L-cysteine, $50 \mathrm{mM} \mathrm{NaEDTA} \mathrm{pH=8,10} \mathrm{ml}$ DS, $10 \mathrm{mM} \mathrm{CaCl} 2,1 \mathrm{~N} \mathrm{HCl}, 100 \mu 110 \mathrm{mg} / \mathrm{ml}$ DnaseI, $100 \mu \mathrm{l}$ Papain) and put on a rotator in 
$37^{\circ} \mathrm{C}$ incubator for 30 minutes. After digestion, the solution was replaced by Inactivation Solution (IS: $10 \mathrm{ml} \mathrm{5 \%} \mathrm{Serum} \mathrm{Medium} \mathrm{(25} \mathrm{ml} \mathrm{Fetal} \mathrm{Bovine} \mathrm{Serum,} 500 \mathrm{ml}$ MEM without Earle's salts and L-glutamine, $7.5 \mathrm{ml} \mathrm{Hi-Glucose} \mathrm{MEM,} 1 \mathrm{ml}$ Mito+Serum Extender), $100 \mu 110 \mathrm{mg} / \mathrm{ml}$ DnaseI, $25 \mathrm{mg}$ BSA) and the hippocampi were settled for 2 minutes. The inactivation solution was removed after the cells had settled. The rest was mixed with 5\% Serum Medium slowly by adding small drops of the medium and applying gentle trituration couple of times. After the larger undigested pieces had settled down, the solution was removed with a sterile Pasteur pipette. Then, the suspension was centrifuged for 5 minutes at $500 \mathrm{~g}$. The supernatant was removed and 1 $\mathrm{ml}$ of 5\% Serum Medium was added per animal. The cells were counted in a Neubauer chamber and 100.000 cells were plated onto each well of Poly-D-lysine (PDL) precoated 24 well plate.

Infection of the cultured neurons with lentivirus expressing specific constructs was done at DIV7. The infected neurons were collected on DIV14 for biochemical analysis. For the infection of a single well of 24 well plate, $1 \mu l$ of large scale virus was used.

The cultures were treated with FUDR (Sigma Aldrich) on DIV5 in order to prevent astrocyte growth and fed on DIV7 by exchanging half of the media for fresh one. Feeding procedure was repeated every 3-7 days.

\subsubsection{Organotypic rat hippocampal slice culture}

Organotypic slice cultures were prepared from P8 Wistar rat hippocampi (Stoppini et al., 1991). The rats were decapitated after being anesthetized with Isoflurane. The hippocampi were carefully isolated in ice cold sterile filtered sucrose cutting buffer (204 mM sucrose, $26 \mathrm{mM} \mathrm{NaHCO}_{3}, 10 \mathrm{mM}$ D-Glucose, $2.5 \mathrm{mM} \mathrm{KCl}, 1 \mathrm{mM} \mathrm{NaH} \mathrm{PO}_{4}{ }^{*} \mathrm{H}_{2} \mathrm{O}, 4$ $\mathrm{mM} \mathrm{MgSO}{ }_{4} * 7 \mathrm{H}_{2} \mathrm{O}, 1 \mathrm{mM} \mathrm{CaCl}{ }_{2} * 2 \mathrm{H}_{2} \mathrm{O}, 4 \mathrm{mM}$ L-ascorbic acid). After removing the meninges, $300 \mu \mathrm{m}$ thick hippocampal slices were cut by using a guillotine slicer. The slices were then separated gently in the sucrose cutting buffer and transferred into sterile filtered and artificial cerebrospinal fluid (ACSF) (119 mM NaCl, $26 \mathrm{mM} \mathrm{NaHCO}$, $20 \mathrm{mM}$ D-Glucose, $2.5 \mathrm{mM} \mathrm{KCl} .1 \mathrm{mM} \mathrm{NaH}_{2} \mathrm{PO}_{4}, 4 \mathrm{mM} \mathrm{MgSO}{ }_{4} * 7 \mathrm{H}_{2} \mathrm{O}, 4 \mathrm{mM} \mathrm{CaCl}{ }_{2} * 2 \mathrm{H}_{2} \mathrm{O}$ ) for recovery for 30 minutes. Both the sucrose cutting buffer and artificial cerebrospinal 
fluid for recovery were bubbled 30 minutes before the slicing, to reach $5 \% \mathrm{CO}_{2}$ and 95\% $\mathrm{O}_{2}$ equilibrium.

Meanwhile, cell culture dishes were prepared with 20\% HK media (28 mM D-Glucose, 51\% BME (Biochrom), 25\% EBSS (1.8 mM CaCl $2 * 2 \mathrm{H}_{2} \mathrm{O}, 1 \mathrm{mM} \mathrm{NaH} \mathrm{PO}_{4} * \mathrm{H}_{3} \mathrm{O}, 0.8 \mathrm{mM}$ $\mathrm{MgSO}_{4} * 7 \mathrm{H}_{2} \mathrm{O}, 116 \mathrm{mM} \mathrm{NaCl}, 26.2 \mathrm{mM} \mathrm{NaHCO}_{3}, 5.4 \mathrm{mM} \mathrm{KCl}, 5 \mathrm{mM}$ D-Glucose), 2.5\% $1 \mathrm{M}$ HEPES (Biochrom), 20\% Horse Serum $\left(55^{\circ} \mathrm{C} 30 \mathrm{~min}\right.$. heat inactivated, Gibco/Invitrogen), 0.5\% Glutamax (Biochrom), $88 \mu \mathrm{g} / \mathrm{ml}$ Ascorbic acid (Sigma), $1 \mu \mathrm{g} / \mathrm{ml}$ Insulin (Sigma), 0.25\% 100x MEM Vitamin (Biochrom), 0.5\% 50x MEM Aminoacid (Biochrom)) on which 5mm $\mathrm{x}$ $5 \mathrm{~mm}$ membrane pieces were placed on plastic inserts with $0.4 \mu \mathrm{m}$ porous membrane (Millipore). After recovery, slices were plated onto the small membrane pieces and the dishes were kept in $37^{\circ} \mathrm{C}$ incubator with equilibrated $5 \% \mathrm{CO}_{2}$ concentration. One day after slicing, the medium was changed for fresh $20 \% \mathrm{HK}$ and the slices were transferred in a $34^{\circ} \mathrm{C}$ incubator with equilibrated $5 \% \mathrm{CO}_{2}$ concentration.

At DIV2, the slices were injected with large scale lentivirus by using Nanoject II injection device (Drummond Scientific, Broomall, USA). Two spots in CA1 region pyramidal cell layer were targeted for the injections and each injection resulted in the emulsion of $9.2 \mu \mathrm{l}$ of concentrated virus solution. For the injection, 3', glass pipettes (Drummond Scientific Company, USA) were used.

Three days after the injection, the slices were fed with 5\% HK medium (28 mM DGlucose, 65\% BME (Biochrom), 25\% EBSS, 2.5\% 1 M HEPES (Biochrom), 5\% Horse Serum $\left(55^{\circ} \mathrm{C} 30 \mathrm{~min}\right.$. heat inactivated, Gibco), 1\% Glutamax (Biochrom), $88 \mu \mathrm{g} / \mathrm{ml}$ Ascorbic acid (Sigma), $1 \mu \mathrm{g} / \mathrm{ml}$ Insulin(Sigma), 0.32\% 100x MEM Vitamin (Biochrom), 0.65\% 50x MEM Aminoacid (Biochrom)) and the feeding procedure was repeated every second day.

\subsubsection{Organotypic mouse hippocampal slice culture}

Mouse hippocampal slices were prepared in a special dissection solution (50 $\mathrm{ml}$ GBSS (Sigma Aldrich), 1\% (v/v) of 45\% D-Glucose and 1\% Penicillin-Streptomycin (Sigma Aldrich) $(\mathrm{v} / \mathrm{v}))$. After the dissection, the slices were recovered at $4^{\circ} \mathrm{C}$ for 30 minutes. The rest of the procedure was carried on as described in section 2.2.5, differing only in the addition of Penicillin-Streptomycin antibiotics into the feeding media (HK20 and HK5). 


\subsubsection{Acute mouse visual cortex slice preparation}

To analyze the subunit composition of NMDA receptors in visual cortex layer 2-3 pyramidal cells, acute visual cortex slices were prepared from P11 - P20 C57BL/6 wild-type or PSD-95 knockout mice (Elias et al., 2006). The solutions were prepared as described by Matta et al., 2011 methods.

The animals were decapitated after isoflurane-mediated anesthesia. The brain was isolated and submerged in ice cold sucrose cutting buffer $(87 \mathrm{mM} \mathrm{NaCl}, 2.5 \mathrm{mM} \mathrm{KCl}, 0.5$ $\mathrm{mM} \mathrm{CaCl}_{2}, 1.25 \mathrm{mM} \mathrm{NaH}_{2} \mathrm{PO}_{4}, 25 \mathrm{mM} \mathrm{NaHCO}_{3}, 25 \mathrm{mM}$ D- Glucose, $75 \mathrm{mM}$ Sucrose). 300 $\mu \mathrm{m}$ thick whole brain slices were cut from two hemispheres in sucrose cutting buffer by using Leica VT 1200S slicing device (Leica, Solmes, Germany). After cutting, the slices were kept in ACSF solution $(119 \mathrm{mM} \mathrm{NaCl}, 25 \mathrm{mM} \mathrm{KCl}, 2.5 \mathrm{mM} \mathrm{CaCl} 2,1 \mathrm{mM}$ $\mathrm{Na}_{2} \mathrm{HPO}_{4}, 25 \mathrm{mM} \mathrm{NaHCO}_{3}, 25 \mathrm{mM} \mathrm{D}$ - Glucose, $1.3 \mathrm{mM} \mathrm{MgSO}_{4}$ ) at $35^{\circ} \mathrm{C}$ for 30 minutes under continuous bubbling with $5 \% \mathrm{CO}_{2}$. After the incubation at $35^{\circ} \mathrm{C}$, the slices were transferred to room temperature and kept for another 30 minutes for the recovery before starting electrophysiological recordings.

\subsection{In vivo injection into mouse visual cortex}

In order to investigate the involvement of PSD-95 in the developmental NMDA receptor subunit switch in visual cortex, in vivo injection technique was applied on P0 C57BL/6 mouse pups. Newborn animals were anesthesized on ice for 10 minutes prior to the injection. After the anesthesia, the animals were stabilized on the injection plate. $65 \mathrm{nl}$ of high titer large scale virus solution, carrying an RNAi construct against mouse PSD-95 or replacement constructs with CDK5 mutants of PSD-95, were injected through glass capillaries into the visual cortex in both hemispheres with Nanoject II injection device (Drummond Scientific, Broomall, USA). The injection was carried on in a pseudo-stereotactic manner.

After the injection, the animals were kept on a heating plate for the recovery. The mother and the pups were then transferred to a new cage. When the animals reached the age of P14, acute brain slices were prepared for electrophysiological recordings. 

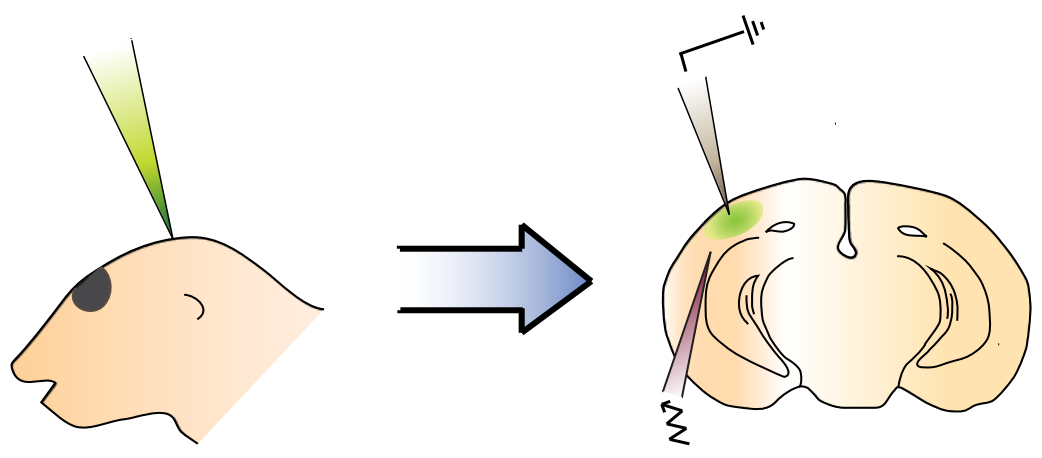

Figure 9: In vivo injection applied on P0 mouse visual cortex.

Lentivirus carrying PSD-95 RNAi or mutant constructs of PSD-95 were injected into the visual cortex of newborn animals. After 2 weeks, the animals were sacrificed to perform electrophysiological recordings on the infected cells in the layer 2-3 of visual cortex electrically stimulated by layer 4 projections.

\section{$2.4 \quad$ Genoytyping}

PSD-95 knockout mice were genotyped prior to electrophysiological experiments. A small tail piece from P0-3 animals were taken as sample and lysed overnight in PBND lysis buffer (10 mM Tris, $50 \mathrm{mM} \mathrm{KCl,} 2.5 \mathrm{mM} \mathrm{MgCl}_{2} * 6 \mathrm{H}_{2} \mathrm{O}, 0.1 \mathrm{mg} / \mathrm{ml}$ Gelatine, $0.45 \%$ (v/v) Nonident P40, 0.45\% (v/v) Tween 20, $\mathrm{pH}=8,3$ ) containing $1.2 \mathrm{mg} / \mathrm{ml}$ Proteinase $\mathrm{K}$ (Roth) at $55^{\circ} \mathrm{C}$ under $1000 \mathrm{rpm}$ agitation or two hours under $1400 \mathrm{rpm}$ agitation. Then the samples were transferred to $99^{\circ} \mathrm{C}$ for 10 minutes in order to inactivate Proteinase $\mathrm{K}$ and centrifuged to remove of the non-lysed particles. The supernatant was separated and used as sample for the PCR. The wild-type gene yielded in a $255 \mathrm{bp}$ long band size, whereas knockout gene showed a 355 bp band size.

Table 6: Primers used for wild-type and PSD-95 knockout animal genotyping.

Primers for genotyping

GKopt fwd2

CAGGTGCTGCTGGAAGAAGG

GKopt rev2

CTACCCTGTGATCCAGAGCTG 
Table 7: Content of the PCR mix used for wild-type and PSD-95 knockout animal genotyping.

PCR Mix

Sample

10x TNK Buffer

dNTP's (2.5 mM each, Bioline)

$50 \mu \mathrm{M}$ Fwd Primer

$50 \mu \mathrm{M}$ Rev Primer

$\mathrm{H}_{2} \mathrm{O}$

Mango Taq polymerase (Bioline)
$2 \mu 1$

$2.2 \mu 1$

$2 \mu 1$

$0.2 \mu \mathrm{l}$

$0.2 \mu 1$

$15.2 \mu \mathrm{l}$

$0.2 \mu \mathrm{l}$

$22 \mu 1$

Table 8: PCR program used for wild-type and PSD-95 knockout animal genotyping.

PCR Program

$\left.\begin{array}{lll}\hline & \text { Time } & \text { Temperature } \\ \text { Preincubation } & 5^{\prime} & 94^{\circ} \mathrm{C} \\ & & \\ \text { Denaturation } & 45^{\prime}, & 94^{\circ} \mathrm{C} \\ \text { Annealing } & 45^{\prime}, & 55^{\circ} \mathrm{C} \\ \text { Elongation } & 1^{\prime} & 72^{\circ} \mathrm{C}\end{array}\right] \times 35$




\subsection{Electrophysiology}

All the electrophysiological recordings were performed using the instruments indicated below.

Table 9: Equipment used for electrophysiological recordings.

Instruments

Microscope

Amplifier

Stimulator

Micromanipulator

Camera
Olympus 51WI (Olympus, Germany)

MultiClamp700B (Molecular Devices, Sunnyvale, CA, USA)

ISO-FLEX (A.M.P.I., Jerusalem, Israel)

MP-225 (Sutter Instrument, Novato, CA, USA)

IR-1000 Infrared CCD Camera (DAGE-MTI, Michigan

City, IN, USA)

\subsubsection{Data acquisition and analysis}

For electrophysiological recordings and data analysis, custom programmed IGOR Pro 6.2 software was used. In all the recordings, the signal was filtered at $4 \mathrm{kHz}$ and digitalized at $10 \mathrm{kHz}$.

Dual whole cell patch-clamp recordings were analyzed by using paired Students ttest. Results are represented as mean values \pm standard error of the mean (SEM). Results presenting $\mathrm{p}$ values smaller than 0.05 were considered statistically significant, and $\mathrm{p}$ values smaller than 0.001 were considered highly significant.

For the analysis of miniature EPSCs, Kolmogorov Smirnov test was used. Visual cortex NMDA receptor recordings were analyzed by one-way ANOVA and post-hoc Tukey test. 


\subsubsection{Basal synaptic transmission recordings in rat hippocampal}

slices

For dual whole cell patch clamp experiments to measure the basal synaptic transmission, hippocampal slices were placed in the recording chamber containing voltage clamp ACSF (119 mM NaCl, $26 \mathrm{mM} \mathrm{NaHCO}_{3}, 20 \mathrm{mM}$ Glucose, $2.5 \mathrm{mM} \mathrm{KCl}, 1 \mathrm{mM}$ $\mathrm{NaH}_{2} \mathrm{PO}_{4}, 4 \mathrm{mM} \mathrm{MgSO}{ }_{4} * 7 \mathrm{H}_{2} \mathrm{O}, 4 \mathrm{mM} \mathrm{CaCl}{ }_{2} * 2 \mathrm{H}_{2} \mathrm{O}, 1-2 \mu \mathrm{M}$ 2-chloroadenosine, $50 \mu \mathrm{M}$ picrotoxin, oxygenated with $5 \% \mathrm{CO}_{2} 30 \mathrm{~min}$ before starting the recording). Picrotoxin was added to isolate only excitatory transmission and 2-chloroadenosine was added to prevent polysynaptic activity in the slices. The chamber was preheated at $32{ }^{\circ} \mathrm{C}$ and kept at the same temperature throughout the recordings with a perfusion speed of $3 \mathrm{ml}$ ACSF/min. For patching, borosilicate KG-33 glass pipettes were used with a resistance of 2.5 - $4 \mathrm{M} \Omega$ filled with cesium-based voltage-clamp internal $(117.5 \mathrm{mM} \mathrm{MeSO} 3 \mathrm{H}$, $10 \mathrm{mM}$ HEPES, $17.75 \mathrm{mM} \mathrm{CsCl}, 10 \mathrm{mM}$ TEA-Cl, $0.25 \mathrm{mM}$ EGTA, 10mM Glucose, 2 $\mathrm{mM} \mathrm{MgCl} 2-6 \mathrm{H} 2 \mathrm{O}, 4 \mathrm{mM} \mathrm{Na} 2 \mathrm{ATP}, 0.3 \mathrm{mM} \mathrm{NaGTP}, \mathrm{CsOH}(50 \% \mathrm{w} / \mathrm{v})$, osm $\cong 290$, $\mathrm{pH}=7$ )

AMPAR EPSCs were recorded at $-60 \mathrm{mV}$ and NMDAR EPSCs at $+40 \mathrm{mV}$. For the quantification, the peak of the AMPAR EPSC amplitude was taken, whereas for NMDAR EPSCs, $60 \mathrm{~ms}$ after the peak value was taken to assure the contribution of only NMDARs and exclude that of AMPARs. 40 sweeps with $5 \mathrm{sec}$ interval were recorded both for AMPAR and NMDAR EPSCs.

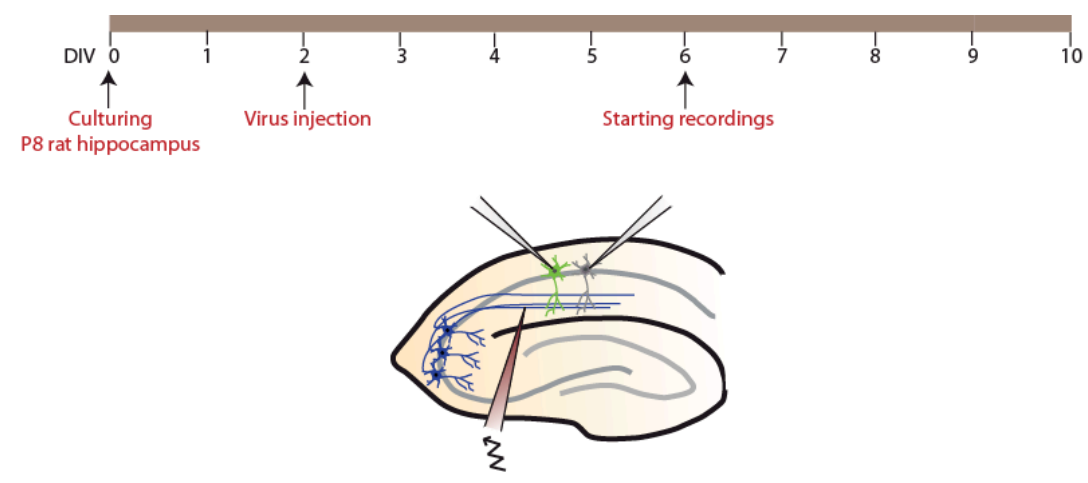

Figure 10: Rat hippocampal slice culture preparation and recording scheme.

Hippocampal slice cultures were prepared from P8 rats. 2 days after the culturing, lentivirus expressing the desired construct was injected into the CA1 region. 4 days after the injection, dual whole cell patch clamp recordings were performed to measure the AMPAR and NMDAR transmission in both infected and control cells by electrically stimulating the Schaffer collaterals. 


\subsubsection{Miniature EPSC recordings in rat hippocampal slices}

The ACSF solution for miniature EPSC (mEPSC) recordings was prepared as described above, only differing in $\mathrm{MgCl}_{2}$ concentration ( $1 \mathrm{mM}$ instead of $4 \mathrm{mM}$ ), the addition of $0.5 \mu \mathrm{M}$ TTX and exclusion of 2-chloroadenosine.

The cells were voltage clamped at $-60 \mathrm{mV}$. For the data analysis, cells which had at least 200 events during a time course of 10 minutes were taken.

\subsubsection{NMDAR EPSC recordings in mouse visual cortex}

After the slices were prepared from mice with different ages, half an hour recovery time was given before the start of recordings. Afterwards, the slices were transferred into the recording chamber containing the same composition of ACSF used for recovery, supplemented with $50 \mu \mathrm{M}$ picrotoxin, $1 \mu \mathrm{M}$ 2-chloroadenosine and $5 \mu \mathrm{M}$ NBQX to isolate NMDAR EPSCs. The recordings were performed at $+40 \mathrm{mV}$ with glass pipette resistances of 4-5 M $\Omega$. The voltage-clamp internal used for the recordings was prepared according to Matta et al., 2011.

The baseline was recorded for 20 minutes and after establishing a stable baseline, 5 $\mu \mathrm{M}$ ifenprodil was washed in to specifically block NR2B content of NMDAR EPSCs. Peak values of the responses were taken as NMDAR excitatory postsynaptic current (EPSC) amplitude. The last 5 minutes of the baseline were taken as the reference point and the recording was continued for 25 minutes after the drug entered the chamber. The responses were normalized to the initial value of the NMDAR EPSCs before the drug treatment.

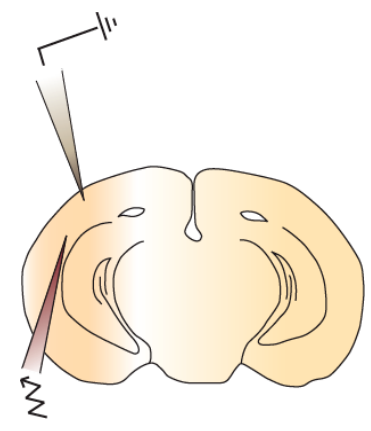

Figure 11: NMDAR EPSC recording in mouse visual cortex. In order to record NMDAR EPSC's, layer 2-3 pyramidal cells in mouse visual cortex from P11-20 animals were patched and clamped at $+40 \mathrm{mV}$. The cells were stimulated electrically through layer 4 connections. 


\subsubsection{Drugs used for pharmacological manipulations on hippocampal and brain slices}

For all the recordings indicated above, as well as the acute and chronic drug treatments on the slices, below listed drugs were used.

Table 10: Concentrations, effects and the sources of the drugs used for electrophysiological recordings.

\begin{tabular}{|c|c|c|c|}
\hline Drug name & Concentration & Effect & Supplier \\
\hline Picrotoxin & $50 \mu \mathrm{M}$ & $\begin{array}{ll}\mathrm{GABA}_{\mathrm{A}} & \text { receptor } \\
\text { blocker } & \end{array}$ & $\begin{array}{l}\text { Ascent } \\
\text { Scientific/Abcam, } \\
\text { Germany }\end{array}$ \\
\hline 2-chloroadenosine & $1-2 \mu \mathrm{M}$ & $\begin{array}{l}\text { Blocking } \\
\text { polysynaptic activity, } \\
\mathrm{A}_{1} \text { adenosine receptor } \\
\text { agonist }\end{array}$ & $\begin{array}{l}\text { Biolog (Bremen, } \\
\text { Germany) }\end{array}$ \\
\hline NBQX & $5 \mu \mathrm{M}$ & $\begin{array}{ll}\text { AMPA } & \text { receptor } \\
\text { blocker } & \end{array}$ & $\begin{array}{l}\text { Ascent } \\
\text { Scientific/Abcam, } \\
\text { Germany }\end{array}$ \\
\hline Ifenprodil & $5 \mu \mathrm{M}$ & $\begin{array}{l}\text { NR2B subunit } \\
\text { blocker }\end{array}$ & \\
\hline DL - TBOA & $100 \mu \mathrm{M}$ & $\begin{array}{l}\text { Glutamate transporter } \\
\text { blocker }\end{array}$ & $\begin{array}{l}\text { Tocris bioscience, } \\
\text { Germany }\end{array}$ \\
\hline TTX & $\begin{array}{l}1 \mu \mathrm{M} \\
\text { (for act. block.) } \\
500 \mathrm{nM} \\
\text { (for mEPSC rec.) }\end{array}$ & $\mathrm{Na}^{+}$channel blocker & $\begin{array}{l}\text { Ascent } \\
\text { Scientific/Abcam, } \\
\text { Germany }\end{array}$ \\
\hline PP2 & $10 \mu \mathrm{M}$ & $\begin{array}{l}\text { Src kinase family } \\
\text { blocker }\end{array}$ & $\begin{array}{l}\text { Sigma-Aldrich } \\
\text { (München, } \\
\text { Germany) }\end{array}$ \\
\hline SFK & $20 \mu \mathrm{M}$ & D1 dopamine receptor & Tocris Biosciences \\
\hline
\end{tabular}




\begin{tabular}{|c|c|c|c|c|}
\hline & & agonist & & (Bristol, UK) \\
\hline PACAP & $10 \mathrm{nM}$ & $\begin{array}{l}\text { Pituitary } \\
\text { cyclase } \\
\text { agonist }\end{array}$ & $\begin{array}{l}\text { adenylate } \\
\text { receptor }\end{array}$ & $\begin{array}{l}\text { Tocris Biosciences } \\
\text { (Bristol, UK) }\end{array}$ \\
\hline MPEP & $25 \mu \mathrm{M}$ & mGluR5 & cker & Abcam, Germany \\
\hline
\end{tabular}

\subsection{Biochemistry}

The chemicals and materials used for biochemical analyses were obtained from Roth (Karlsruhe, Germany), Serva (Heidelberg, Germany), Biochrom (Berlin, Germany), Roche (Mannheim, Germany), Whatman/GE Healthcare (Maidstone, UK), Sigma Aldrich (München, Germany) and Merck (Darmstadt, Germany).

\subsubsection{Dissociated neuron culture sample collection}

Western Blot samples were collected from rat or mouse dissociated neuron cultures of

hippocampus or cortex. Neurons were first washed with PBS containing $\mathrm{Mg}^{+2}$ and $\mathrm{Ca}^{+2}$ and then scraped out from culture dishes in 2x SDS sample buffer (3.3\% Tris- $\mathrm{HCl}, 3.4 \%$ Tris, $8 \%$ SDS, $0.02 \%$ Sucrose, 0.03\% EDTA, 0.02\% bromophenol blue, 0.27\% DTT (w/v)). After collection, the samples were boiled at $100^{\circ} \mathrm{C}$ for 5 minutes and then cooled down to room temperature.

\subsubsection{Co-immunoprecipitation assay}

In order to test specific protein-protein interactions, co-immunoprecipitation assay was used. After transfecting HEK293T cells with desired constructs and establishing the expression, the cells were washed once with PBS without $\mathrm{Mg}^{+2}$ and $\mathrm{Ca}^{+} 2$. The cells were collected in RIPA lysis buffer $\left(50 \mathrm{mM} \mathrm{NaH}_{2} \mathrm{PO}_{4}, 150 \mathrm{mM} \mathrm{NaCl}, 1 \%\right.$ Triton X-100, $0.1 \%$ SDS, $0.5 \%$ deoxycholic acid, $2 \mathrm{mM}$ EDTA and Roche protease inhibitor cocktail, $\mathrm{pH}=$ 7.4) and rocked at $4^{\circ} \mathrm{C}$ for 1 hour. After 1 hour, the lysates were centrifuged at 15000 rpm for 30 minutes at $0^{\circ} \mathrm{C}$. The clean supernatant was pre-washed with Protein $\mathrm{A} / \mathrm{G}-$ conjugated beads for 1 hour at $4^{\circ} \mathrm{C}$ to prevent any unspecific binding during the immunoprecipitation procedure. The supernatant was then centrifuged at $7000 \mathrm{rpm}$ and 
$4^{\circ} \mathrm{C}$ for 10 minutes. After the centrifugation, $1 \mu \mathrm{g}$ of antibody was added into each sample to pull-down the selected protein. The binding was established via over-night incubation at $4^{\circ} \mathrm{C}$. The day after, Protein A- (for rabbit originated antibodies) or ProteinG-conjugated (for mouse originated antibodies) beads were added onto the antibody-bound samples. Another incubation step was applied at $4^{\circ} \mathrm{C}$ for 1 hour. The antibody and bead bound proteins were pulled down at $7000 \mathrm{rpm}$ for 5 minutes at $4^{\circ} \mathrm{C}$. The precipitates were washed 3 times with washing buffer $(10 \mathrm{mM}$ Tris/HCl, $1 \mathrm{mM}$ EDTA, $250 \mathrm{mM} \mathrm{NaCl}, 0.5 \% \mathrm{TX}-100, \mathrm{pH}=7.4)$ and finally once with $1 \mathrm{X}$ TBS $(\mathrm{pH}=7.4)$. Lastly, 2X SDS sample buffer was added on the bead bound proteins and the samples were boiled at $60^{\circ} \mathrm{C}$ for 5 minutes.

\subsubsection{Subcellular fractionation}

Preparation of the crude synaptosome and PSD fractions were done from mice cortices which were homogenized in homogenization buffer $(10 \mathrm{ml} /$ one mouse cortex, $320 \mathrm{mM}$ sucrose, 10 mM HEPES/NaOH, pH 7.4) (Cho et al., 1992; Schlüter et al., 1999). After the homogenization the samples were centrifuged for 10 minutes at $1000 \mathrm{~g}$ at $0^{\circ} \mathrm{C}$ and the supernatant was isolated. A second step of centrifugation was performed on the supernatant for 15 minutes at $15000 \mathrm{~g}$ at $0^{\circ} \mathrm{C}$ to isolate crude synaptosomes. The pellet was resuspended in the resuspension buffer $(50 \mathrm{mM} \mathrm{NaF}, 50 \mathrm{mM}$ HEPES/NaOH, pH7.4, 1 mM EDTA, $0.2 \mathrm{mM} \mathrm{NaVO4,} \mathrm{protease} \mathrm{inhibitor} \mathrm{cocktail} \mathrm{(Roche))} \mathrm{for} \mathrm{a} \mathrm{final}$ concentration of $1 \mu \mathrm{g} / \mu \mathrm{l}$. Resuspended samples were treated with $0.5 \%$ Triton X100 for 15 minutes and were centrifuged at $32000 \mathrm{~g}$ for 20 minutes at $0^{\circ} \mathrm{C}$. The supernatant formed the Triton 100x soluble (1TS) fraction and the pellet Triton 100x insoluble fraction (1TP). The 1TP fraction was resuspended in the resuspension buffer and was treated with 3\% N-lauroylsarcosine for 10 minutes on ice. The treated suspension was centrifuged at $200000 \mathrm{~g}$ for 1 hour at $0^{\circ} \mathrm{C}$. The pellet formed the N-lauroylsarcosine insoluble PSD fraction (TSP) and the supernatant N-lauroylsarcosine soluble fraction (TSS). TSP fraction was used as the sample for western-blotting after being resuspended in 2\% SDS. Protein concentrations were measured with BCA kit (Pierce). 


\subsubsection{Western blotting}

For the biochemical analysis of the proteins isolated from dissociated neuron cultures or HEK293T cells, samples were run by SDS-PAGE on Bis-Tris gels (Shapiro et al., 1967; Graham et al., 2005) followed by western-blotting (Towbin et al., 1979). The gels for sample running were prepared according to the following recipes.

Table 11: Recipes of Bis-Tris gels used for Western-blotting.

7.5\% Separating Gel (375 mM Bis-Tris, $p H=6.4)$

\begin{tabular}{ll}
\hline $\mathrm{H}_{2} \mathrm{O}$ & $9 \mathrm{ml}$ \\
AMBA & $5 \mathrm{ml}$ \\
$3.5 \%$ gel buffer & $6 \mathrm{ml}$ \\
TEMED & $25 \mu 1$ \\
$10 \%$ APS & $60 \mu 1$ \\
& \\
$3.75 \%$ Stacking Gel (125 mM Bis-Tris, pH= 6.4$)$ \\
\hline $\mathrm{H}_{2} \mathrm{O}$ & $6.2 \mathrm{ml}$ \\
AMBA & $1 \mathrm{ml}$ \\
$3.5 \%$ gel buffer & $0.8 \mathrm{ml}$ \\
TEMED & $20 \mu 1$ \\
$10 \%$ APS & $48 \mu 1$ \\
\hline
\end{tabular}

$3.5 \%$ gel buffer: $1.25 \mathrm{M}$ Bis-Tris, $\mathrm{pH}=6.5-6.8$

The samples were run at $120 \mathrm{~V}$ for 2 hours to achieve a good resolution of the high molecular weight protein bands. The proteins were transferred onto nitrocellulose membranes in the transfer buffer ( $25 \mathrm{mM}$ Tris, $191 \mathrm{mM}$ Glycin, 20\% Methanol) at $250 \mathrm{~mA}$ for two hours. The membranes were prestained with Panceau $\mathrm{S}$ to confirm the success of the protein transfer. The membranes were blocked either in 5\% milk powder in TBS$\mathrm{T}$ for regular antibody blotting $(20 \mathrm{mM}$ Tris/ $\mathrm{HCl}, 140 \mathrm{mM} \mathrm{NaCl}, 0.1 \%$ Tween $20, \mathrm{pH}=7.6)$ or in 3\% BSA in TBS-T for the phospho-antibody blotting. Primary antibody solutions were prepared in $2.5 \%$ milk powder or $3 \%$ BSA containing TBS-T. Primary antibody 
binding was performed at $4{ }^{\circ} \mathrm{C}$ for 2 hours or overnight. The membrane was washed three times for 5 minutes with TBS-T and the secondary antibody binding was done at room temperature for 2 hours.

Afterwards, the membrane was washed three times for 10 minutes with TBS-T and once for 10 minutes with TBS (20 mM Tris/ $\mathrm{HCl}, 140 \mathrm{mM} \mathrm{NaCl}, \mathrm{pH}=7.6)$. Visualization of the protein bands and the analysis of the band intensity were done by using Odyssey Infrared Imaging System (Li-COR Biosciences, Bad Homburg, Germany). Proteins were visualized at 700 or $800 \mathrm{~nm}$ according to the secondary antibody used.

\subsubsection{Antibodies used for Immunoblotting}

To probe the membranes for specific target proteins, following antibodies were used according to the indicated dilutions (Table 12). 
Table 12: List of the antibodies used for immunoblotting.

\begin{tabular}{|c|c|c|}
\hline Antibodies & Dilution & Company \\
\hline \multicolumn{3}{|l|}{ Primary antibodies } \\
\hline$\alpha$-PSD-95 (mouse) & $1: 10000$ & Neuromab \\
\hline$\alpha-$ Src kinase (rabbit) & $1: 2000$ & Genescript \\
\hline$\alpha-N R 2 B$ (mouse) & $1: 2000$ & Neuromab \\
\hline$\alpha$-phospho NR2B (Y1472) (rabbit) & $1: 1000$ & Cell Signalling \\
\hline$\alpha$-Mortalin (mouse) & $1: 10000$ & Neuromab \\
\hline$\alpha-\mathrm{NR} 2 \mathrm{~A}$ (mouse) & $1: 5000$ & Millipore \\
\hline$\alpha$-PSD-93 (mouse) & $1: 2000$ & Neuromab \\
\hline$\alpha$-SAP102 (mouse) & $1: 2000$ & Neuromab \\
\hline$\alpha$-SAP97 (mouse) & $1: 2000$ & Neuromab \\
\hline$\alpha$-GluA1 (mouse) & $1: 2000$ & Abcam \\
\hline$\alpha-\beta$-tubulin (mouse) & $1: 2000$ & Sigma Aldrich \\
\hline$\alpha$-GDI (mouse) & & (Schlüter et al., 1999) \\
\hline$\alpha$-synaptophysin (mouse) & & (Schlüter et al., 1999) \\
\hline \multicolumn{3}{|l|}{ Secondary antibodies } \\
\hline$\alpha$-mouse Alexa Flour 680 (goat) & $1: 10000$ & Invitrogen \\
\hline$\alpha$-rabbit Alexa Flour 680 (goat) & $1: 10000$ & Invitrogen \\
\hline$\alpha$-mouse IR 800 (goat) & $1: 10000$ & Li-COR Biosciences \\
\hline$\alpha$-rabbit IR 800 (goat) & $1: 10000$ & Li-COR Biosciences \\
\hline
\end{tabular}




\section{Results}

\subsection{The effect of CDK5 phosphorylation mutants of PSD- 95 on basal synaptic transmission in rat hippocampal slice culture}

\subsubsection{Optimization of the CDK5 phosphorylation mutant constructs of PSD-95}

CDK5 phosphorylation of PSD-95 has been shown to regulate the synaptic clustering of PSD-95 in hippocampal neurons (Morabito et al., 2004). Moreover, the phosphorylation status of PSD-95 had been associated with an indirect role to regulate the NR2B subunit containing NMDA receptor surface expression (Zhang et al., 2008). However, these results were gathered from biochemical and imaging assays, leaving an open question concerning the understanding of the functional consequence of such a regulation on synaptic transmission.

In order to assess this question, I used the rat organotypic hippocampal slice culture as a model system and first tested the AMPAR and NMDAR transmission properties of CA1 region neurons mimicking different CDK5 phosphorylation states of PSD-95 by dual whole cell patch-clamp technique.

This method involved the replacement of the endogenous PSD-95 with a recombinant version, either mimicking the CDK5 phosphorylation $(\operatorname{sh} 95+$ p95D3::GFP) or mimicking the non-phosphorylated version (sh95 + p95A3::GFP) through the substitution of T19, S25, S35 aminoacids with aspartate and alanine residues respectively.

Initially, I expressed the above-mentioned recombinant PSD-95 proteins as well as the pure wild-type expression of PSD-95 with a GFP-tag under the influence of WPRE transcription enhancer cassette (Figure 5). The expression of the mutants showed significant increases in AMPAR mediated transmission $(\operatorname{sh} 95+$ p95A3::GFP, uninfected: $-108.7 \pm 13.8$ pA, infected: $-206.7 \pm 24.4$ pA, p $<0.05$; sh95 + p95D3::GFP, uninfected: $-90.2 \pm 7.9 \mathrm{pA}$, infected: $-155.4 \pm 16.7 \mathrm{pA}, \mathrm{p}<0.001$ ). 
AMPAR

A

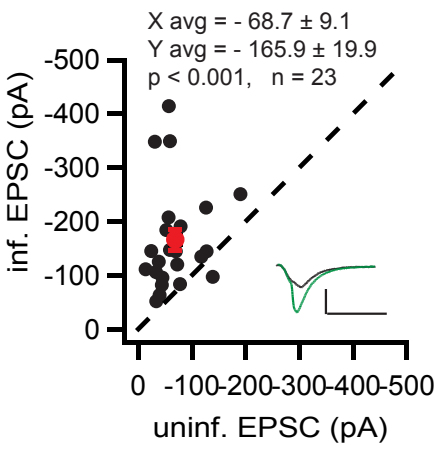

NMDAR

p95::GFP

B

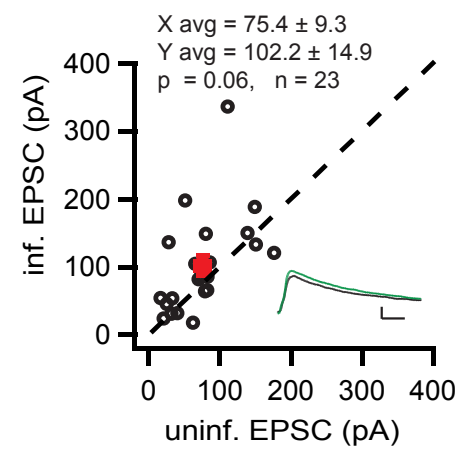

sh95 + p95A3::GFP
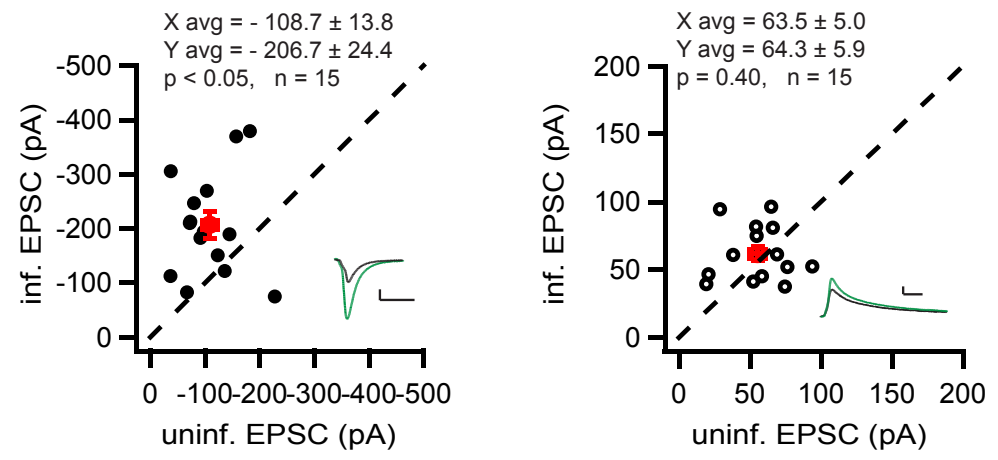

C sh95 + p95D3::GFP
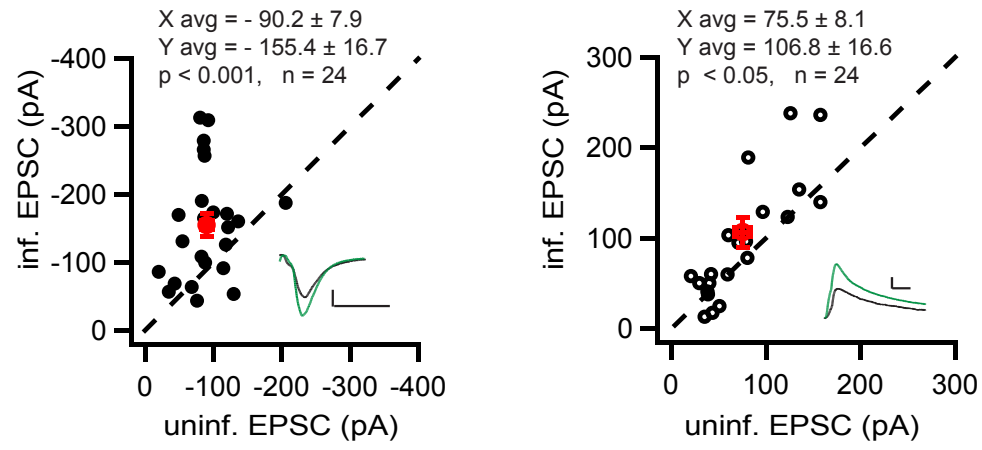

Figure 12: Expression of GFP-tagged CDK5 mutants of PSD-95 under the influence of WPRE transcription enhancer results in overexpression effect comparable to wild-type PSD-95 overexpression in CA1 region of rat hippocampus.

(A) PSD-95 overexpression in CA1 region hippocampal cells resulted in a significant increase in AMPAR transmission $(p<0.001)$ without a change in NMDAR transmission $(p=0.06)$.

(B) The replacement of endogenous PSD-95 by the non-phosphorylatable mutant increased the AMPAR transmission significantly $(\mathrm{p}<0.05)$ but not the NMDAR transmission $(\mathrm{p}=0.40)$.

(C) There was a highly significant increase in the AMPAR transmission $(p<0.001)$ as well as a significant increase in NMDAR transmission $(\mathrm{p}<0.05)$ when phospho-mimicking PSD-95 was expressed in the presence of the shRNA against endogenous PSD-95.

(The scale bars correspond to $50 \mathrm{pA}$ and $25 \mathrm{~ms}$. Green traces indicate the infected and the black traces indicate the control cell responses. The red marker corresponds to the average of all data points on the plot.) 
Only the phospho-mimicking mutant displayed a significant increase in NMDAR transmission in comparison to the control cells (sh95 + p95A3::GFP, uninfected: $63.5 \pm$ 5.0 pA, infected: $64.3 \pm 5.9$ pA, p $<0.40$; sh95 + p95D3::GFP, uninfected: $-90.2 \pm 7.9$ $\mathrm{pA}$, infected: $-155.4 \pm 16.7 \mathrm{pA}, \mathrm{p}<0.05$ ) (Figure $12 \mathrm{~B}, \mathrm{C}$ ). The increases observed in AMPAR transmission levels were comparable to the increase observed with pure PSD95 overexpression (AMPAR, uninfected: $-68.7 \pm 9.1 \mathrm{pA}$, infected: $165.9 \pm 19.9 \mathrm{pA}$, $\mathrm{p}<0.001$; NMDAR, uninfected: $75.4 \pm 9.3 \mathrm{pA}$, infected: $102.2 \pm 14.9 \mathrm{pA}, \mathrm{p}=0.06$ ) (Figure $12 \mathrm{~A}$ ).

As both of the constructs showed an increase in AMPAR transmission level comparable to the overexpression of PSD-95, a critical point was to differentiate the overexpression effect from the mutant effects on the basal synaptic transmission. In order to achieve this, the expression level of the constructs was lowered by excising the WPRE enhancer cassette from the vector backbones (Figure 6). At the same time, I generated a wild-type PSD-95 replacement construct without the WPRE cassette as a control. This approach brought the AMPAR and NMDAR transmission strength of the cells expressing wild-type PSD-95 replacement (sh95 + p95::GFP without WPRE) to the same level as controls (AMPAR, uninfected: $-55.6 \pm 7.8 \mathrm{pA}$, infected: $46.8 \pm 8.5$ $\mathrm{pA}, \mathrm{p}=0.84$; NMDAR, uninfected: $22.5 \pm 4.1 \mathrm{pA}$, infected: $19.5 \pm 3.4 \mathrm{pA}, \mathrm{p}=0.35$ ) (Figure $13 \mathrm{~A}$ ). On the other hand, the increase in AMPAR transmission observed with the non-phosphorylatable mutant was absent once the expression levels were lowered, and there was no change in the NMDAR transmission (sh95 + p95A3::GFP without WPRE) (AMPAR, uninfected: $-48.2 \pm 6.5 \mathrm{pA}$, infected: $45.3 \pm 7.2 \mathrm{pA}, \mathrm{p}=0.72$; NMDAR, uninfected: $31.7 \pm 8.4 \mathrm{pA}$, infected: $28.5 \pm 5.8 \mathrm{pA}, \mathrm{p}=0.56$ ) (Figure $13 \mathrm{~B}$ ). The phospho-mimicking mutant, on the other hand, $(\operatorname{sh} 95+$ p95D3::GFP without WPRE) preserved a significant increase in AMPAR transmission but lost the enhancement in NMDAR transmission (AMPAR, uninfected: $-31.8 \pm 3.4 \mathrm{pA}$, infected: $45.3 \pm 5.5 \mathrm{pA}, \mathrm{p}=0.72$; NMDAR, uninfected: $29.6 \pm 7.8 \mathrm{pA}$, infected: $25.1 \pm 5.7 \mathrm{pA}$, $\mathrm{p}=0.56$ ) (Figure $13 \mathrm{C}$ ). These results clearly demonstrate the importance of the expression levels, as overexpression might mask the mutation effect. 

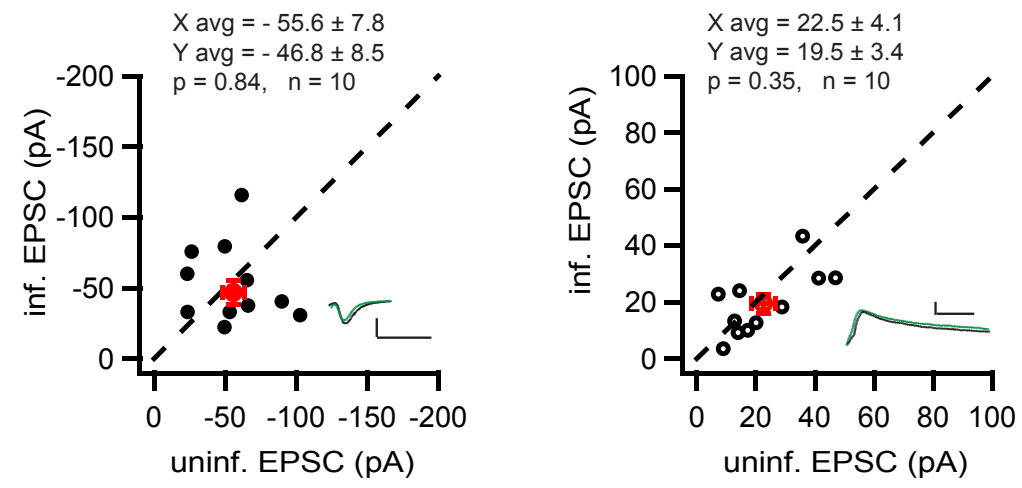

B

sh95 + p95A3::GFP without WPRE
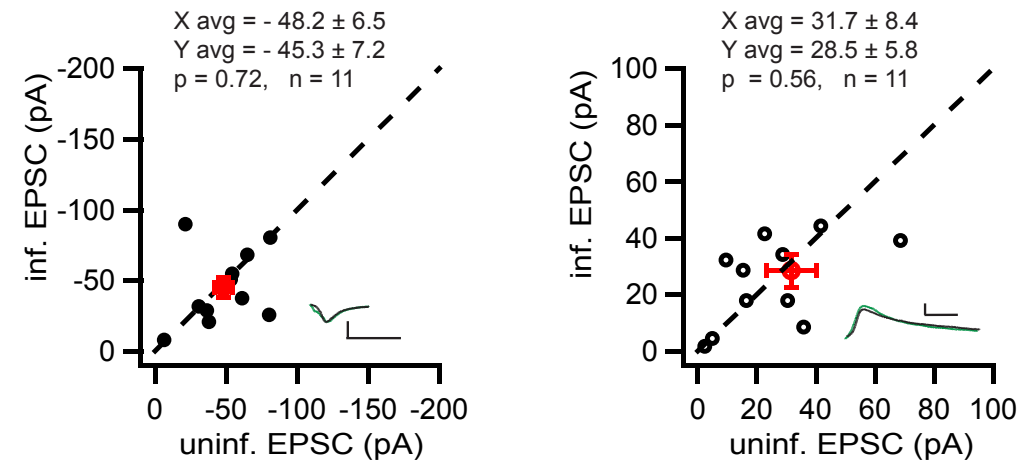

C
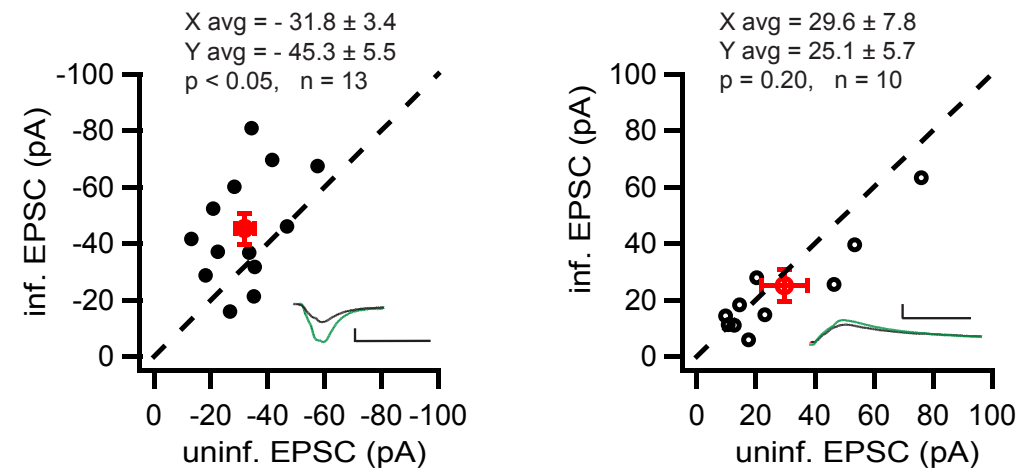

Figure 13: Expression of GFP-tagged CDK5 mutants of PSD-95 without the WPRE transcription enhancer distinguishes the mutation effect from the overexpression effect.

(A) PSD-95 wild-type replacement in CA1 region hippocampal neurons without the WPRE cassette exhibited AMPAR $(p=0.84)$ and NMDAR transmission $(p=0.35)$ as the same level as the control cells.

(B) The replacement of endogenous PSD-95 by the non-phosphorylatable mutant without the WPRE cassette showed no change in AMPAR $(p=0.72)$ and NMDAR responses $(p=0.56)$.

(C) The replacement of endogenous PSD-95 by the phospho-mimicking mutant without the WPRE cassette resulted in a significant increase in AMPAR $(\mathrm{p}<0.05)$ but not in NMDAR transmission $(\mathrm{p}=0.20)$.

(The scale bars correspond to $50 \mathrm{pA}$ and $25 \mathrm{~ms}$. Green traces indicate the infected and the black traces indicate the control cell responses. The red marker corresponds to the average of all data points on the plot.) 
In parallel to the electrophysiological recordings, I tested the binding ability of PSD-95 to the constitutively active Src kinase (Y527F) (Polte and Hanks, 1997), since the control of NMDA receptor transmission was indicated to involve Src kinase interaction with PSD-95 (Zhang et al., 2008). In order to test the binding ability, I expressed both GFP-tagged PSD-95 and active Src kinase in HEK293T cells. After expression, Src kinase was immunoprecipitated and tested for the interaction with GFPtagged PSD-95.

Interestingly, there was no PSD-95 band observed in the pull-down assay (Figure 14A). To test whether the GFP-tag may interfere with the Src kinase and PSD-95 interaction, HEK293T cells were transfected with active Src kinase and non-tagged PSD-95. Surprisingly, this combination resulted in the successful coimmunoprecipitation of PSD-95 and Src kinase (Figure 14B).
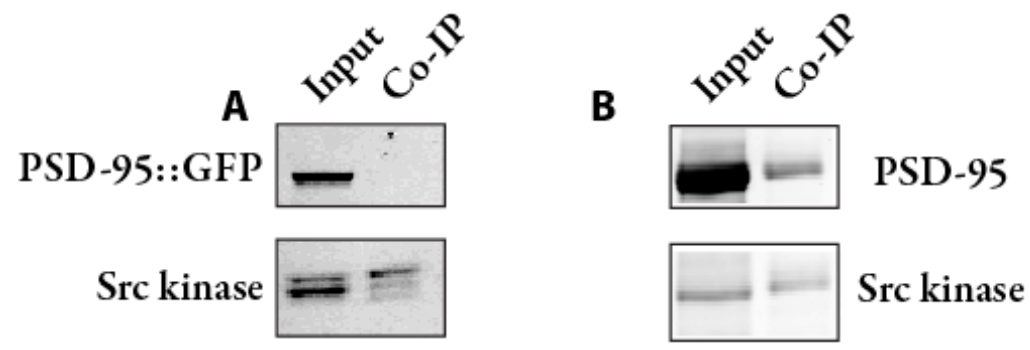

Figure 14: The C-terminal GFP-tag of PSD-95 interferes with the PSD-95 - Src kinase interaction.

(A) HEK293T cells were transfected with GFP-tagged PSD-95 and constitutively active Src kinase (Y527F). Immunoprecipitation was done with Src kinase antibody. There was no coimmunoprecipitation observed with Src kinase and PSD-95.

(B) The transfection of HEK293T cells with non-tagged PSD-95 and active Src kinase enabled the co-immunoprecipitation of PSD-95 with Src kinase. Immunoprecipitation was done with Src kinase antibody.

Due to the results obtained with the GFP-tag, which seemed to interfere with the PSD-95 - Src kinase interaction and also the effect of expression levels on the synaptic transmission strength, which can potentially mask the mutation effects, a new set of lentiviral constructs were generated (Figure 7). The new constructs enabled endogenous levels of PSD-95 expression by excluding the WPRE transcripton enhancer cassette from the vector. In addition, an IRES (internal ribosome entry site) sequence was introduced between the PSD-95 and GFP coding sequences so that untagged PSD-95 
can be expressed and infected cells can still be highlighted with GFP. In addition to the electrophysiological recordings, I tested the expression levels of the replacement constructs in dissociated neuron cultures. The expression of the shRNA against endogenous PSD-95 results in a 90\% knock-down efficiency (data not shown but see (Schlüter et al., 2006)). Exclusion of WPRE cassette from the vector clearly prevents overexpression such that the expression of PSD-95 in the bicistronic construct results in a similar level as the endogenous PSD-95. However, it is not possible to differentiate the bands of the endogenous PSD-95 from the recombinant form of sh95 + p95A3viGFP and sh95 + p95D3viGFP constructs (Figure 15).

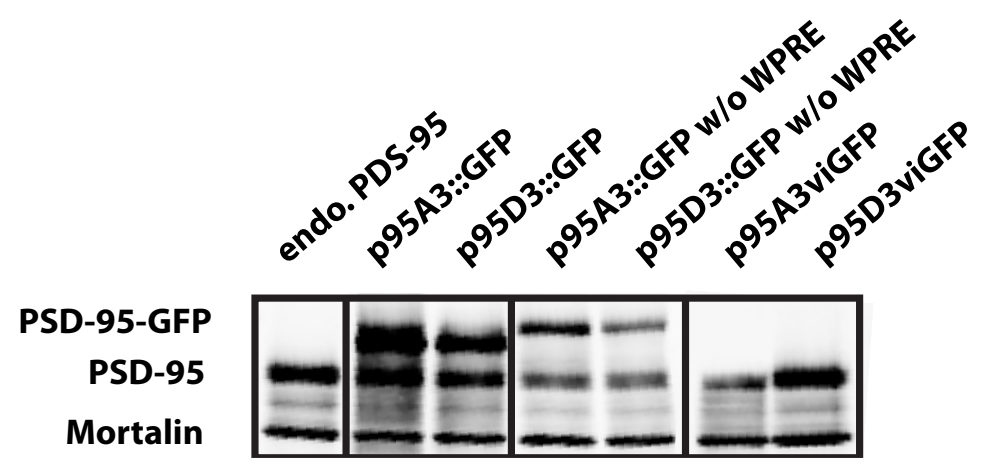

Figure 15: Expression levels of CDK5 phosphorylation mutants of PSD-95 in dissociated neuron culture extracts.

Dissociated mouse neuron cultures prepared from P0 mice were infected at DIV7 with the indicated constructs, all of which contain shRNA against endogenous PSD-95. Proteins were extracted from the cultures on DIV14 and were immunoblotted by PSD-95 and mortalin antibodies.

\subsubsection{AMPAR and NMDAR transmission properties of CDK5 mutants of PSD-95 in CA1 region rat hippocampal neurons}

To ensure that the bicistronic construct could maintain the endogenous levels of PSD95 expression and that its expression does not affect the AMPAR and NMDAR transmission per se, wild-type PSD-95 replacement without WPRE and GFP-tag was recorded as a control for the electrophysiological measurements.

Indeed, replacing endogenous PSD-95 with wild-type PSD-95 (sh95 + p95viGFP) did not result in any change in AMPAR and NMDAR transmission in comparison to control cells (AMPAR, uninfected: $-60.9 \pm 7.1 \mathrm{pA}$, infected: $75.4 \pm 8.0 \mathrm{pA}, \mathrm{p}=0.13$; NMDAR, uninfected: $48.4 \pm 6.6 \mathrm{pA}$, infected: $54.1 \pm 7.1 \mathrm{pA}, \mathrm{p}=0.29)$ (Figure 16A). 
The recordings were repeated for the non-phosphorylatable $(\operatorname{sh} 95+$ p95A3viGFP) and the phospho-mimicking (sh95 + p95D3viGFP) mutants of PSD-95. There was no change observed with the non-phosphorylatable mutant regarding the AMPAR and NMDAR transmission (AMPAR, uninfected: $-76.6 \pm 9.5$ pA, infected: $89.6 \pm 11.6 \mathrm{pA}$, $\mathrm{p}=0.25$; NMDAR, uninfected: $52.6 \pm 12.3 \mathrm{pA}$, infected: $60.5 \pm 11.9 \mathrm{pA}, \mathrm{p}=0.29)$, but the phospho-mimicking mutant demonstrated significant increases for both of the components (AMPAR, uninfected: $-64.1 \pm 7.6$ pA, infected: $89.9 \pm 10.7$ pA, p $<0.05$; NMDAR, uninfected: $52.5 \pm 9.5 \mathrm{pA}$, infected: $84.1 \pm 15.5 \mathrm{pA}, \mathrm{p}<0.05)$ (Figure 16B,C). The NMDAR transmission mediated by the GFP-tagged and non-tagged phosphomimicking mutant was significantly different. This data indicates that the GFP-tag disrupts the effect of the phospho-mimicking mutant on NMDAR transmission. 
AMPAR

A

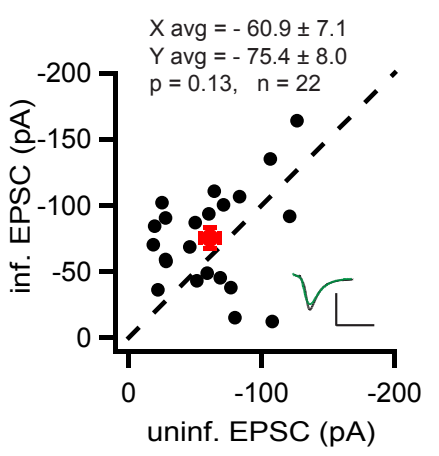

NMDAR

sh95 + p95viGFP

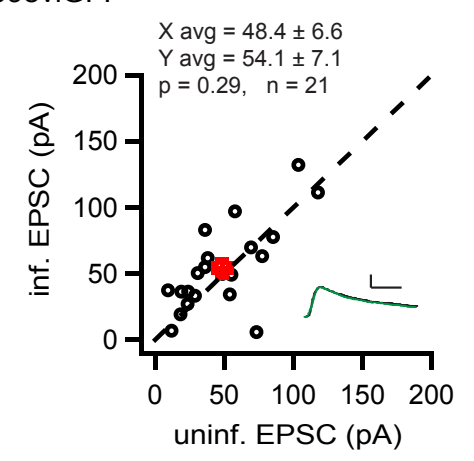

B sh95 + p95A3viGFP
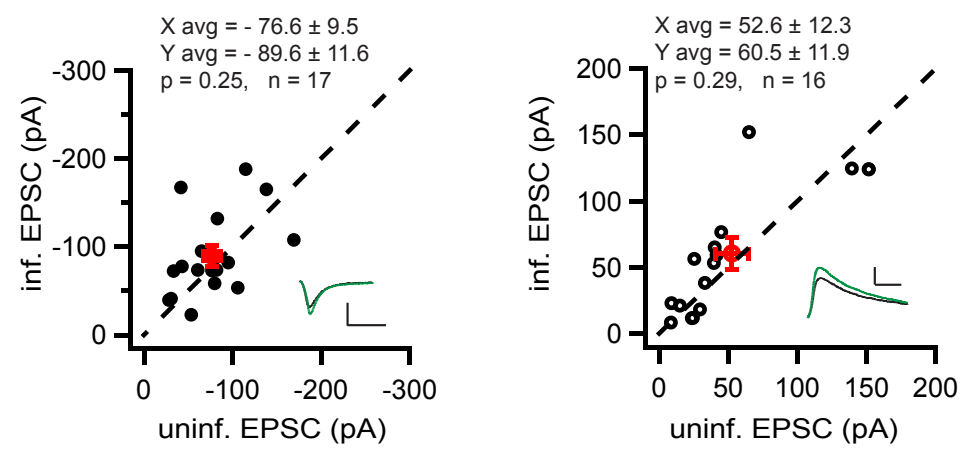

C
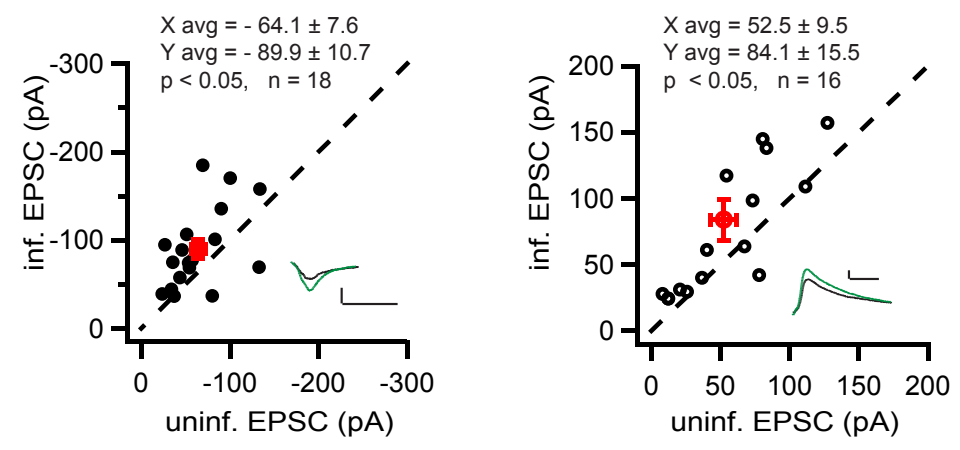

Figure 16: The AMPAR and NMDAR transmission is significantly increased with the non-tagged phospho-mimicking mutant of PSD-95 under endogenous levels of expression.

(A) Replacing the endogenous PSD-95 with wild-type PSD-95 did not alter the AMPAR ( $\mathrm{p}=0.13$ ) and NMDAR transmission ( $\mathrm{p}=0.29)$ of the neurons, when endogenous level of expression is established and GFP expression was separated.

(B) The replacement of the endogenous PSD-95 by the non-phosphorylatable mutant of PSD-95 resulted in no change for both AMPAR $(\mathrm{p}=0.25)$ and NMDAR transmission $(\mathrm{p}=0.29)$, similar to the wild-type replacement.

(C) Replacing the endogenous PSD-95 by the phospho-mimicking mutant of PSD-95 significantly increased both AMPAR $(\mathrm{p}<0.05)$ and NMDAR $(\mathrm{p}<0.05)$ transmission.

(The scale bars correspond to $50 \mathrm{pA}$ and $25 \mathrm{~ms}$. Green traces indicate the infected and the black traces indicate the control cell responses. The red marker corresponds to the average of all data points on the plot.) 


\subsubsection{Activity manipulation of endogenous CDK5 in CA1 region neurons}

To check whether the endogenous CDK5 activity would yield the same effects as the PSD-95 mutant constructs, I over-expressed the CDK5 co-activators p25 and p35 to increase its endogenous activity (Tsai et al., 1994; Patrick et al., 1999). On the other hand, to block CDK5 activity, I overexpressed the dominant-negative mutant of CDK5 (DNK5). The results showed no change in AMPAR and NMDAR transmission between the p35 (AMPAR, uninfected: $-46.1 \pm 6.5 \mathrm{pA}$, infected: $53.0 \pm 6.8 \mathrm{pA}, \mathrm{p}=0.46$; NMDAR, uninfected: $34.5 \pm 6.8$ pA, infected: $42.8 \pm 7.5$ pA, p=0.13), p25 (AMPAR, uninfected: $-86.2 \pm 13.9$ pA, infected: $66.3 \pm 7.3 \mathrm{pA}, \mathrm{p}=0.11$; NMDAR, uninfected: $70.5 \pm 19.4$ pA, infected: $45.9 \pm 8.5$ pA, p=0.08), DNK5 (AMPAR, uninfected: $-80.4 \pm$ 10.9 pA, infected: $84.5 \pm 10.6 \mathrm{pA}, \mathrm{p}=0.76$; NMDAR, uninfected: $48.7 \pm 6.9 \mathrm{pA}$, infected: $41.6 \pm 7.2 \mathrm{pA}, \mathrm{p}=0.19$ ) expressing neurons and control cells (Figure 17). Expression of the constructs was confirmed by the immunoblotting of CDK5, p25 and p35 in rat hippocampal dissociated neuron culture extracts infected with either of the construct. The result of the DNK5 expression was consistent with the nonphosphorylatable mutant expression, as both of the expressions did not alter the AMPAR and NMDAR transmission. However, the effect of p35 and p25 expression, which showed no change in AMPAR and NMDAR responses, was different from the phospho-mimicking mutant expression. This might either point to an artificial effect observed with the phospo-mimicking mutant construct or inefficient activation of the endogenous CDK5. 
AMPAR NMDAR

A

DNK5viGW
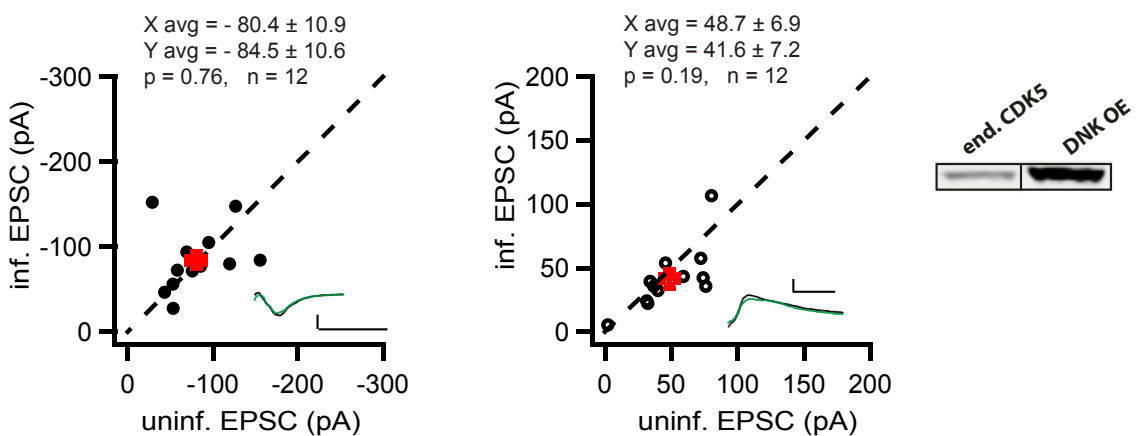

B

p25viGW
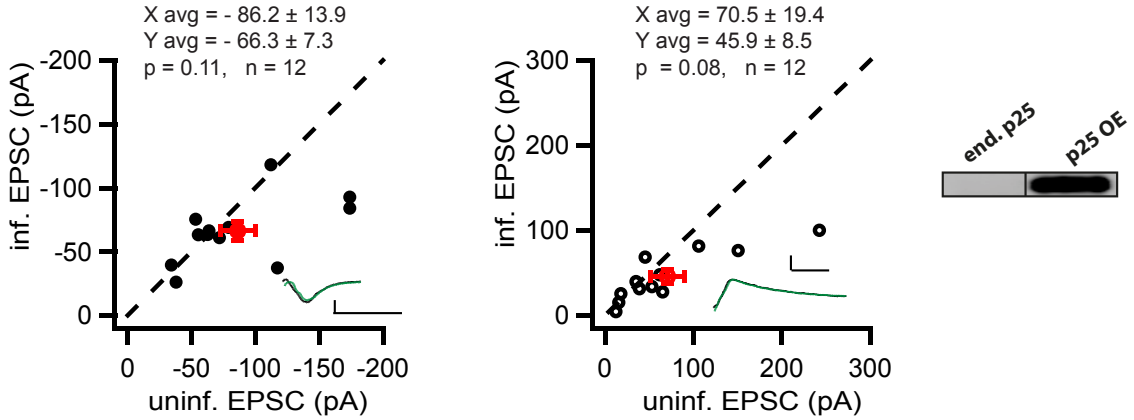

C

p35viGW
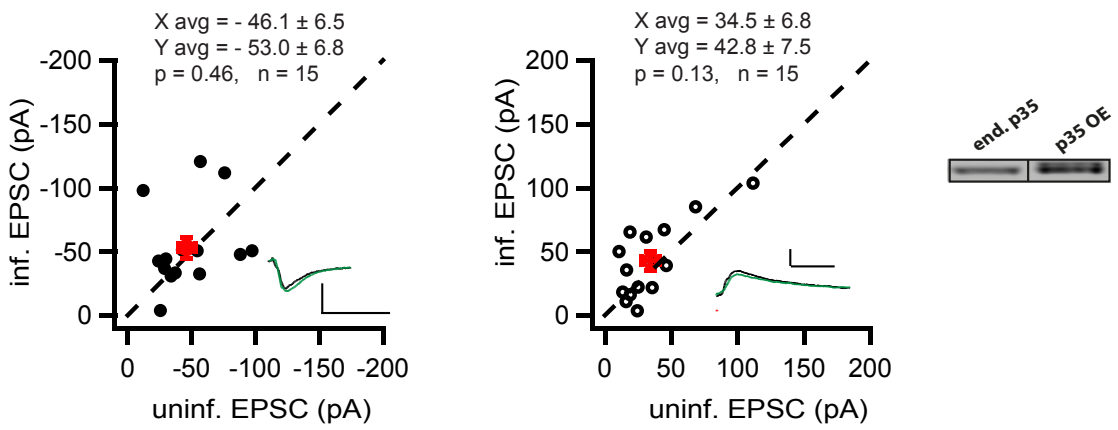

Figure 17: Overexpression of CDK5 co-activators p25 and p35 and a dominant-negative form of CDK5 (DNK5) do not alter the AMPAR and NMDAR transmission in CA1 region neurons.

(A) Expression of a dominant-negative CDK5 construct (DNK5) did not change AMPA $(p=0.76)$ and NMDA receptor responses $(p=0.19)$ in comparison to control cells. Rat hippocampal dissociated neuron culture extracts show the expression levels of endogenous CDK5 and overexpressed DNK5 probed with CDK5 antibody.

(B) Expression of CDK5 co-activator $\mathrm{p} 25$ did not change AMPA $(\mathrm{p}=0.11)$ and NMDA receptor responses $(p=0.08)$ in comparison to control cells. Rat hippocampal dissociated neuron culture extracts show the expression levels of endogenous and over-expressed p25 probed with p35 antibody.

(C) Expression of CDK5 co-activator $\mathrm{p} 35$ did not change AMPA $(\mathrm{p}=0.46)$ and NMDA receptor responses $(p=0.13)$ in comparison to control cells. Rat hippocampal dissociated neuron culture extracts show the expression levels of endogenous and over-expressed p35 probed with p35 antibody.

(The scale bars correspond to $50 \mathrm{pA}$ and $25 \mathrm{~ms}$. Green traces indicate the infected and the black traces indicate the control cell responses. The red marker corresponds to the average of all data points on the plot.) 


\subsubsection{Involvement of Src kinase family in AMPAR and NMDAR transmission mediated by CDK5 phosphorylation mutants of PSD-95}

According to the study from Zhang et al., 2008, Src kinase was suggested to play a role in the regulation of the GluN2B-containing NMDA receptor surface expression. This regulation was established by the phosphorylation of Y1472 residue on the GluN2B subunit $\mathrm{C}$ terminus by Src kinase and it was dependent on the phosphorylation state of PDS-95 by CDK5. In addition, Src kinase family members were indicated to phosphorylate C terminus of AMPAR GluA2 subunit at the residue Y876 (Hayashi and Huganir, 2004). Due to the involvement of Src kinase family members in both AMPAR and NMDAR phosphorylation, I tested the possibility of their involvement in AMPAR and NMDAR transmission of neurons expressing CDK5 phosphorylation mutants of PSD-95. The recordings were performed in the presence of the selective Src kinase family blocker PP2 and both AMPAR and NMDAR transmission were measured. The slices were pretreated with $10 \mu \mathrm{M}$ PP2 for 20 minutes before the recording and the drug was kept in the recording chamber throughout the recordings.

There was no difference between the AMPAR and NMDAR transmission properties of non-phosphorylatable mutant expressing and control cells (AMPAR, uninfected: $-49.6 \pm 11.6 \mathrm{pA}$, infected: $49.0 \pm 7.9 \mathrm{pA}, \mathrm{p}=0.96$; NMDAR, uninfected: $105.4 \pm 19.4 \mathrm{pA}$, infected: $110.8 \pm 23.0 \mathrm{pA}, \mathrm{p}=0.44$ ) (Figure 18A). However, the application of Src kinase family blocker (PP2) abolished the significant increases in AMPAR and NMDAR transmission observed with the phospho-mimicking mutant of PSD-95 (AMPAR, uninfected: $-80.7 \pm 8.4$ pA, infected: $95.9 \pm 9.5 \mathrm{pA}, \mathrm{p}=0.18$; NMDAR, uninfected: $79.3 \pm 12.6 \mathrm{pA}$, infected: $64.3 \pm 12.7 \mathrm{pA}, \mathrm{p}=0.47$ ) indicating that Src kinase family members are indeed involved in the AMPAR and NMDAR transmission mediated by phospho-mimicking mutant of PSD-95 (Figure 18B). 

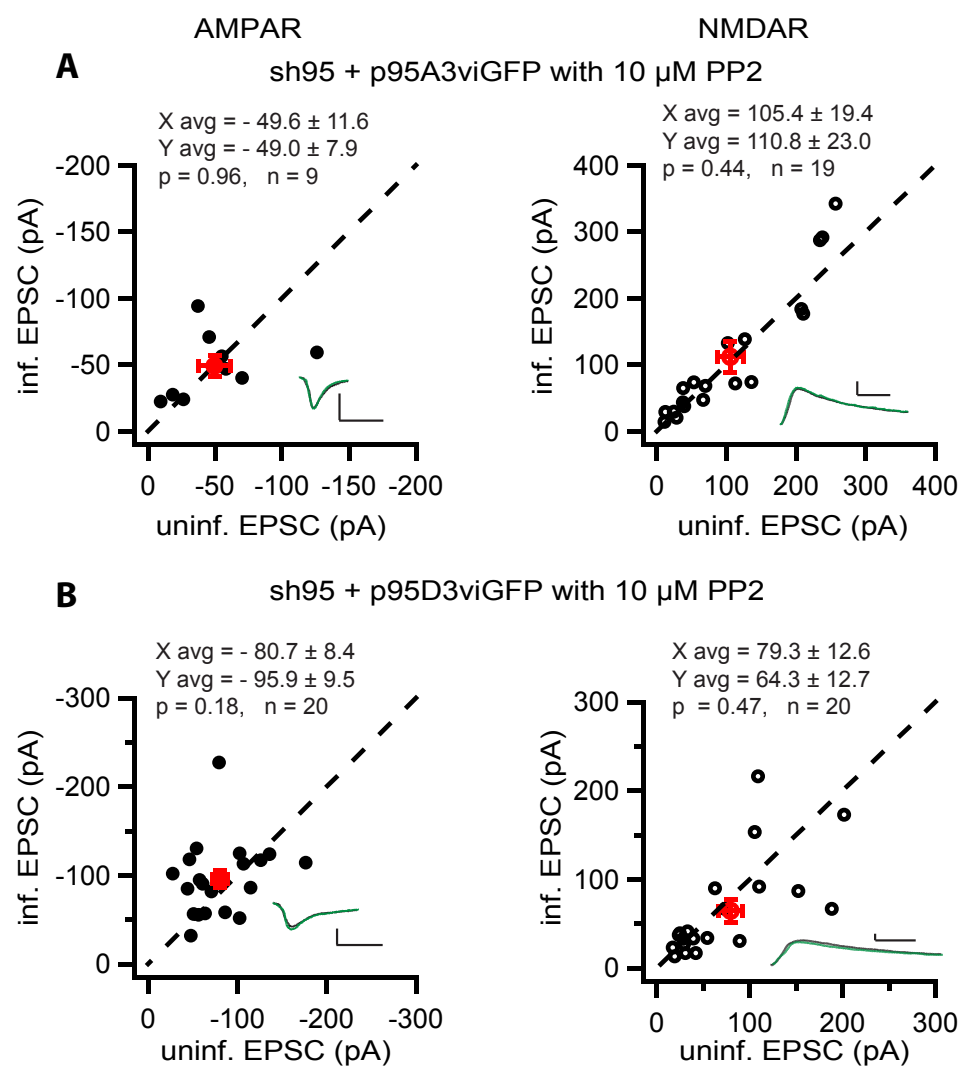

Figure 18: Src kinase family members are involved in the significant increase of AMPAR and NMDAR transmission observed with the phospho-mimicking mutant of PSD-95 in CA1 region neurons.

(A) Application of Src kinase family inhibitor PP2 did not change AMPAR $(\mathrm{p}=0.96)$ and NMDAR transmission $(\mathrm{p}=0.44)$ of non-phosphorylatable mutant expressing cells in comparison to control cells.

(B) Application of Src kinase family inhibitor PP2 abolished the significant increase in AMPAR $(\mathrm{p}=0.18)$ and NMDAR transmission $(\mathrm{p}=0.47)$ observed with the phospho-mimicking mutant of PSD-95.

(The scale bars correspond to $50 \mathrm{pA}$ and $25 \mathrm{~ms}$. Green traces indicate the infected and the black traces indicate the control cell responses. The red marker corresponds to the average of all data points on the plot.)

Src family kinases were shown to phosphorylate both GluN2A and GluN2B subunits of NMDA receptors (Köhr and Seeburg, 1996; Zheng et al., 1998; Nakazawa et al., 2001; Vissel et al., 2001; Takasu et al., 2002). In order to understand whether the increase in NMDAR transmission of phospho-mimicking mutant is subunit-specific, I subjected the slices to $5 \mu \mathrm{M}$ ifenprodil, a GluN2B subunit specific blocker. The slices were pre-incubated with ifenprodil for 20-30 minutes prior to the recording. The drug was kept in the chamber during the experiments. Simultaneous recording of the 
NMDAR responses from untreated and treated slices revealed a GluN2B subunit specific effect of the phospho-mimicking mutant. In the absence of the drug, NMDAR responses were significantly higher than the control cells (uninfected: $97.1 \pm 13.2 \mathrm{pA}$, infected: $144.2 \pm 22.3 \mathrm{pA}, \mathrm{p}<0.05)$. However, with ifenprodil application this effect vanished (uninfected: $130.8 \pm 13.4 \mathrm{pA}$, infected: $117.9 \pm 14.1 \mathrm{pA}, \mathrm{p}=0.36$ ), implying that the enhancement in NMDAR transmission is dependent on GluN2B-containing NMDA receptors.

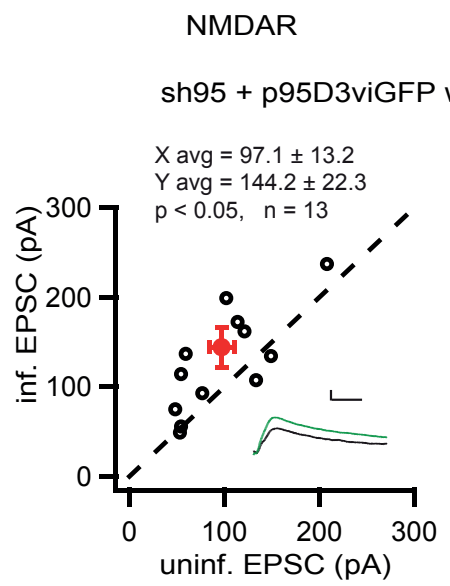

NMDAR

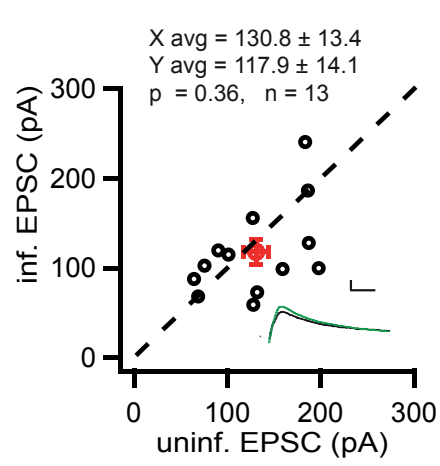

Figure 19: The increase in NMDAR response observed with phospho-mimicking mutant is GluN2B subunit specific in CA1 region neurons.

Slices without ifenprodil treatment showed a significant increase in NMDAR responses with the phospho-mimicking mutant expression $(\mathrm{p}<0.05)$. Incubating the slices in $5 \mu \mathrm{M}$ ifenprodil for $20-30$ minutes abolished this increase $(\mathrm{p}=0.36)$.

(The scale bars correspond to $50 \mathrm{pA}$ and $25 \mathrm{~ms}$. Green traces indicate the infected and the black traces indicate the control cell responses. The red marker corresponds to the average of all data points on the plot.)

Since the increase in the NMDAR transmission of the phospho-mimicking mutant expressing cells is GluN2B dependent, I checked whether this enhancement is due to an increased phosphorylation of the Y1472 residue on GluN2B subunit. I infected dissociated hippocampal neuron cultures with the wild-type replacement construct as well as the CDK5 phosphorylation mutant replacement constructs and immunoblotted GluN2B subunit with the phospho-Y1472 specific antibody. In addition, I blocked the activity of Src kinase family members with $10 \mu \mathrm{M}$ PP2 in the cultures and checked the additive effect of Src kinase blockade and PSD-95 mutants on GluN2B Y1472 phosphorylation. The analysis showed that, there was no difference in the total GluN2B 
levels in wild-type PSD-95 and phosphorylation mutant expressing cells (one-way ANOVA, F(2,6)=0.09, p=0.91; WtviG: $1 \pm 0.08$; A3viG: $1.03 \pm 0.20$, D3viG: $0.95 \pm$ $0.02, \mathrm{~N}=3$ ) (Figure 20C). However, there was a tendency for an increase in Y1472 phosphorylation levels of phospho-mimicking mutant expressing neurons (one-way ANOVA, $\mathrm{F}(2,6)=0.50, \mathrm{p}=0.63$; WtviG: $1 \pm 0.19$; A3viG: $1.02 \pm 0.25$, D3viG: $1.41 \pm$ 0.46, N=3) (Figure 20B). These results suggest that the NMDAR transmission enhancement observed with phospho-mimicking mutant might be dependent on GluN2B Y1472 phosphorylation. At the same time, application of PP2 in all cases reduced GluN2B phosphorylation dramatically (Figure 20A).

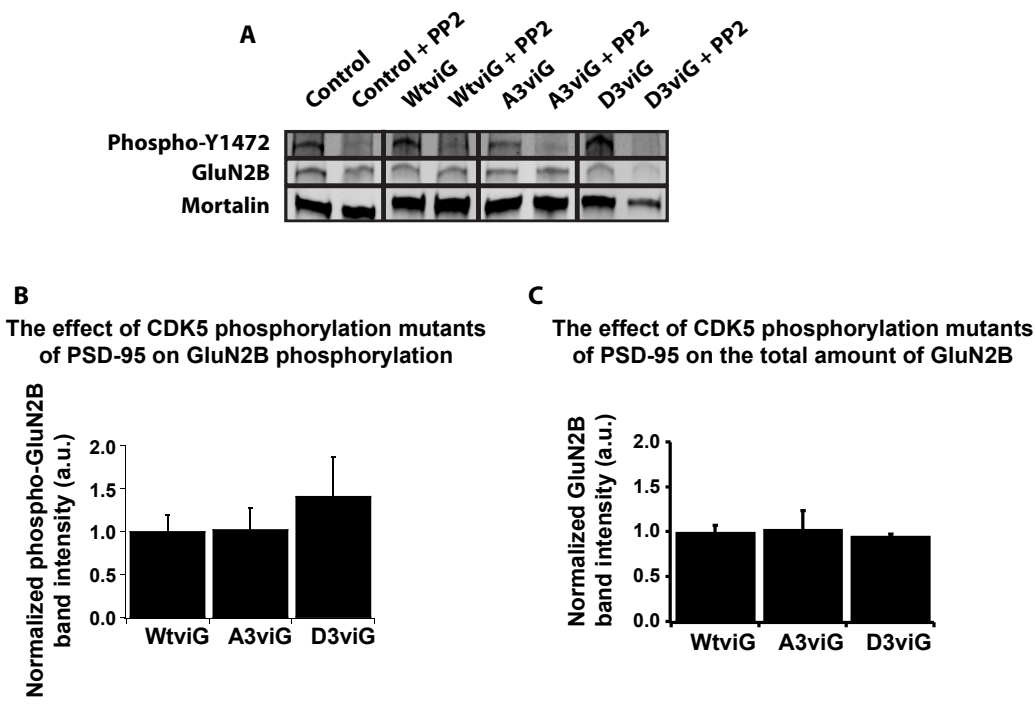

Figure 20: Phospho-mimicking mutant has a tendency to enhance Y1472 phosphorylation on GluN2B without changing total levels of GluN2B.

Dissociated mouse hippocampal neuron cultures were infected with wild-type PSD-95 and CDK5 phosphorylation mutant replacement constructs at DIV7. Cultures were collected at DIV14 and the extracts were immunoblotted with phospho-Y1472, GluN2B and mortalin antibodies. GluN2B and phospho-Y1472 bands were normalized to the mortalin band.

(A) Phospho-Y1472, GluN2B and mortalin bands are shown from cells extracts of control and virus infected neurons, in the presence and absence of $10 \mu \mathrm{M}$ PP2.

(B) Quantification was performed by normalizing the phospho-Y1472 band intensity to the mortalin band intensity. The results from the CDK5 phosphorylation mutant expressing cells are normalized to wild-type PSD-95 expressing cells. Bar graphs represent the mean \pm SEM. (oneway ANOVA, F $(2,6)=0.50$, p=0.63; WtviG: $1 \pm 0.19$; A3viG: $1.02 \pm 0.25$, D3viG: $1.41 \pm 0.46$, $\mathrm{N}=3$ for all)

(C) Quantification was performed by normalizing the total GluN2B band intensity to the mortalin band intensity. The results from the CDK5 phosphorylation mutant expressing cells are normalized to the wild-type PSD-95 expressing cells. Bar graphs represent the mean \pm SEM. (one-way ANOVA, F(2,6)=0.09, $\mathrm{p}=0.91$; WtviG: $1 \pm 0.08$; A3viG: $1.03 \pm 0.20, \mathrm{D} 3 \mathrm{viG}: 0.95 \pm$ $0.02, \mathrm{~N}=3$ for all) 
The results collected from the phospho-mimicking mutant expressing cells indicate a GluN2B subunit specific NMDAR enhancement, which is mediated by the Src kinase family. However, it is not clear which member of the Src kinases plays a role in this regulation. A recent study by Yang et al., 2012 suggested two possible mechanisms of NMDAR enhancement, one in which dopamine 1 (D1) receptors specifically activate Fyn kinase and another in which pituitary adenylate cyclase activating peptide 1 receptors (PAC1Rs) selectively activate Src kinase. Stimulation of these receptors resulted in the activation of endogenous Src and Fyn kinases and phosphorylation of GluN2A and GluN2B subunits, respectively (Yang et al., 2012). Taking advantage of these pathways, I aimed to dissect the role of Src and Fyn kinases in the regulation of NMDAR transmission displayed by the phospho-mimicking PSD-95 mutant. I used 1 $\mathrm{nM}$ pituitary adenylate cyclase activating peptide 38 (PACAP38) to stimulate PAC1 receptors thus Src kinase, and $10 \mu \mathrm{M}$ SKF81297 to stimulate D1 receptors hence Fyn kinase. The slices were perfused with the drugs in the recording chamber for 10-15 minutes prior to and during recordings.

Application of PACAP38 modified the AMPAR and NMDAR transmission mediated by the non-phosphorylatable mutant and control cells to the same extent (AMPAR, uninfected: $-76.3 \pm 8.7 \mathrm{pA}$, infected: $-97.9 \pm 10.9 \mathrm{pA}, \mathrm{p}=0.15$; NMDAR, uninfected: $47.9 \pm 8.7 \mathrm{pA}$, infected: $46.6 \pm 5.8 \mathrm{pA}, \mathrm{p}=0.77$ ) (Figure $21 \mathrm{~A}$ ). However, the significant increase in NMDAR responses observed with the phospho-mimicking mutant was this time absent. AMPAR transmission was still significantly higher than the control cells, which implies that PAC1R activation affected AMPAR, but not NMDAR transmission of infected and control cells in the same way as the phosphomimicking mutant (AMPAR, uninfected: $-71.4 \pm 8.0 \mathrm{pA}$, infected: $118.9 \pm 18.8 \mathrm{pA}$, $\mathrm{p}<0.05$; NMDAR, uninfected: $71.1 \pm 17.3 \mathrm{pA}$, infected: $84.8 \pm 20.3 \mathrm{pA}, \mathrm{p}=0.18$ ) (Figure $21 \mathrm{~B}$ ). 


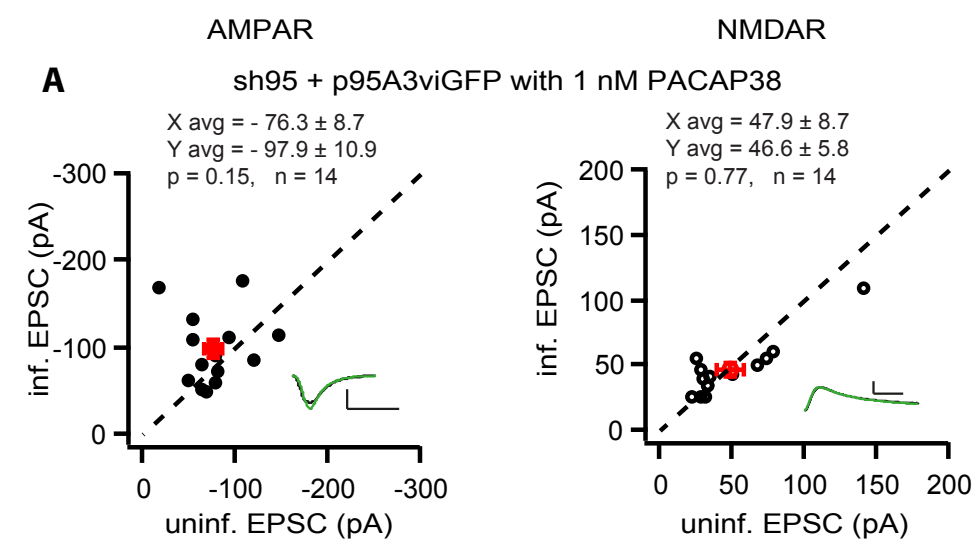

B $\quad$ sh95 + p95D3viGFP with $1 \mathrm{nM}$ PACAP38
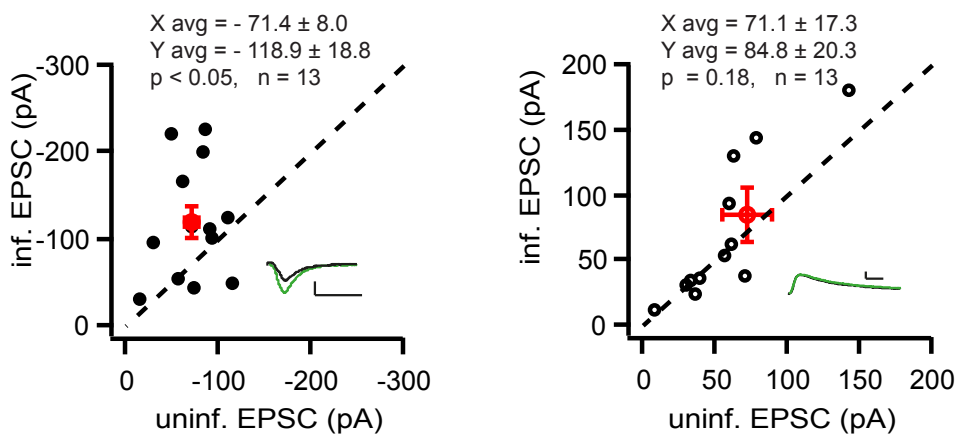

Figure 21: Activation of PAC1Rs, known to activate Src kinase, brings the NMDAR transmission of phospho-mimicking mutant expressing cells to the same level as control cells in CA1 region neurons.

(A) PAC1R activation affected the non-phosphorylatable mutant expressing and control cells in the same rate. There was no difference observed between the infected and control cells regarding AMPAR ( $\mathrm{p}=0.15)$ and NMDAR transmission $(\mathrm{p}=077)$.

(B) PAC1R activation brought the NMDAR transmission of control cells to the same level as phospho-mimicking mutant expressing cells $(\mathrm{p}=0.18)$. AMPAR responses of infected cells were still significantly higher than the control cells $(\mathrm{p}<0.05)$.

(The scale bars correspond to $50 \mathrm{pA}$ and $25 \mathrm{~ms}$. Green traces indicate the infected and the black traces indicate the control cell responses. The red marker corresponds to the average of all data points on the plot.)

Interestingly, application of $10 \mu \mathrm{M}$ SFK81297 to activate D1 receptors thus Fyn kinase, significantly enhanced the AMPAR and NMDAR responses of the nonphosphorylatable mutant expressing cells (AMPAR, uninfected: $-67.6 \pm 10.0 \mathrm{pA}$, infected: $-117.7 \pm 16.2 \mathrm{pA}, \mathrm{p}<0.001$; NMDAR, uninfected: $71.0 \pm 14.2 \mathrm{pA}$, infected: $84.4 \pm 14.5 \mathrm{pA}, \mathrm{p}<0.05$ ) (Figure $22 \mathrm{~A}$ ). On the other hand, the increase in AMPAR and NMDAR transmission observed with the phospho-mimicking mutant was absent after the drug application (AMPAR, uninfected: $-84-2 \pm 2-8 \mathrm{pA}$, infected: $-114.1 \pm 23.1 \mathrm{pA}$, 
$\mathrm{p}=0.26$; NMDAR, uninfected: $67.7 \pm 8.4 \mathrm{pA}$, infected: $62.9 \pm 14 \mathrm{pA}, \mathrm{p}=0.61$ ) (Figure 22 B). These results suggest that eliminating the CDK5 phosphorylation on PSD-95 enhances the effects of D1 receptor activation. In contrast, the effect of the phosphomimicking mutant and D1 receptor activation are not additive, indicating that there might be an overlap between these two pathways.

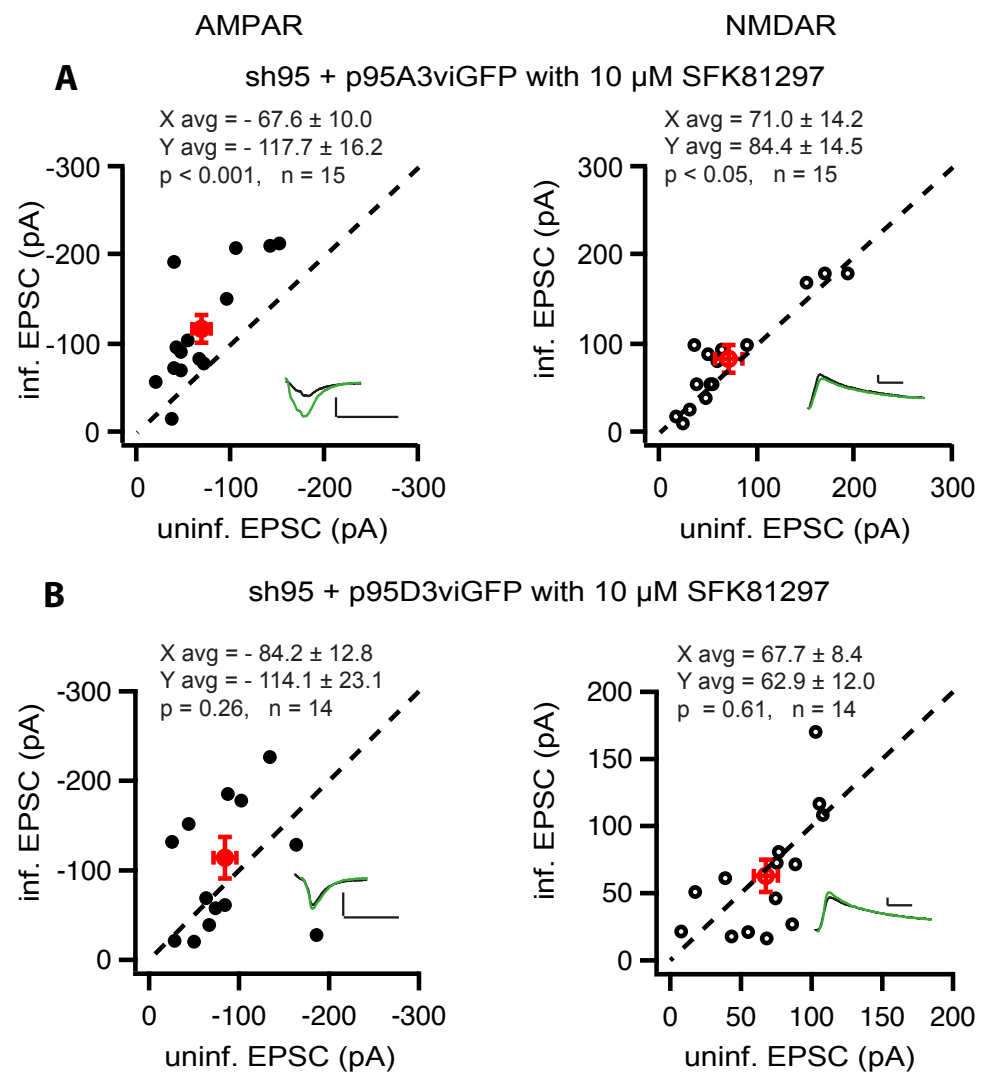

Figure 22: Activation of D1 dopamine receptors, known to activate Fyn kinase, enhances both AMPAR and NMDAR transmission of non-phosphorylatable mutant expressing cells, at the same time bringing the AMPAR and NMDAR transmission of control cells to the same level as phosphomimicking mutant expressing cells in CA1 region neurons.

(A) Application of SFK81297 has a bigger effect on non-phosphorylatable mutant expressing cells than the control cells, regarding AMPAR $(\mathrm{p}<0.001)$ and NMDAR transmission $(\mathrm{p}<0.05)$.

(B) Application of SFK81297 brings AMPAR ( $p=0.26)$ and NMDAR transmission $(p=0.61)$ of control cells to the same level as phospho-mimicking mutant cells.

(The scale bars correspond to $50 \mathrm{pA}$ and $25 \mathrm{~ms}$. Green traces indicate the infected and the black traces indicate the control cell responses. The red marker corresponds to the average of all data points on the plot.) 


\subsubsection{Activity-dependent effects of CDK5 phosphorylation mutants of PSD-95 on AMPAR and NMDAR transmission}

As AMPA receptor transmission can be modulated via NMDA receptor activation, one important question raised was whether the increase in AMPAR responses of phosphomimicking mutant expressing neurons is due to the increased levels of NMDAR transmission. In order to test this possibility, I injected the rat hippocampal organotypic slices with a phospho-mimicking mutant expressing virus. After two days of infection, I subjected the slices to $5 \mu \mathrm{M}$ ifenprodil for two days in order to block GluN2Bcontaining receptors before the virus expression is fully established. At the end of the two days, I measured the AMPAR and NMDAR transmission in the presence of $5 \mu \mathrm{M}$ ifenprodil. The results showed that the significant increase in AMPAR responses observed with the mutant was still present and, as expected, the increase in NMDAR responses was absent due to the ifenprodil treatment (AMPAR, uninfected: $-36.8 \pm 2.9$ pA, infected: $-56.6 \pm 7.9 \mathrm{pA}, \mathrm{p}<0.05$; NMDAR, uninfected: $18.0 \pm 3.2 \mathrm{pA}$, infected: $24.1 \pm 5.3 \mathrm{pA}, \mathrm{p}=0.18$ ) (Figure 23). This data suggests that the increase in AMPAR transmission is not due to the enhancement in NMDAR transmission.

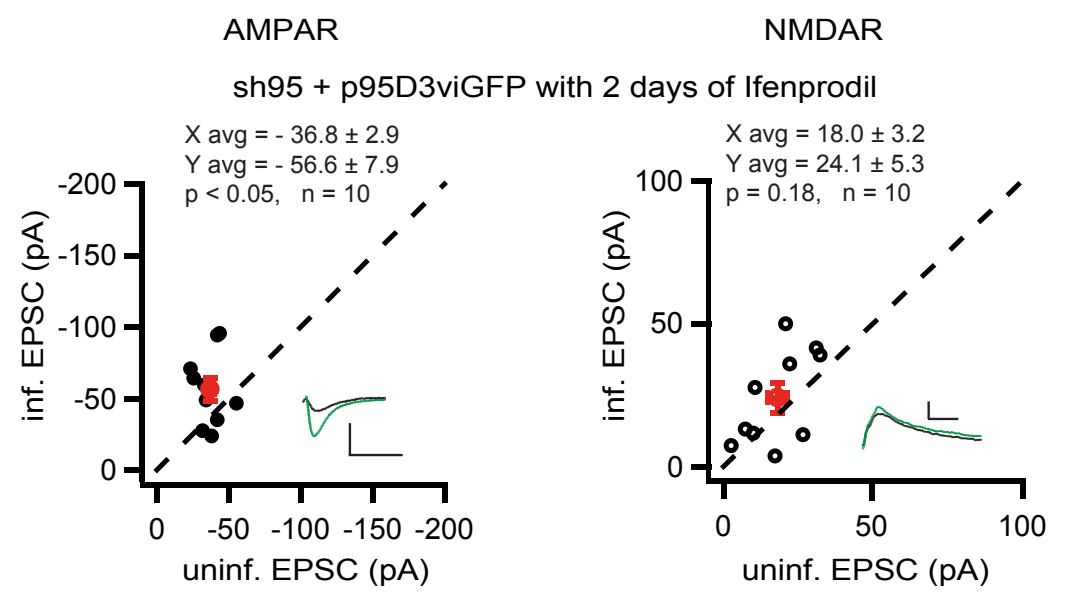

Figure 23: The significant increase in AMPAR transmission of phospho-mimicking mutant expressing cells is not due to the enhancement in NMDAR transmission in CA1 region neurons. Exposing the slices at DIV 4 to $5 \mu \mathrm{M}$ ifenprodil for 2 days did not inhibit the significant increase in AMPA receptor transmission $(\mathrm{p}<0.05)$. NMDA receptor responses were not different between the control and infected cells due to ifenprodil treatment $(\mathrm{p}=0.18)$.

(The scale bars correspond to $50 \mathrm{pA}$ and $25 \mathrm{~ms}$. Green traces indicate the infected and the black traces indicate the control cell responses. The red marker corresponds to the average of all data points on the plot.) 
A recent study by Zhang et al., 2012 demonstrated that the overexpression effect of PSD-95, leading to the enhancement of AMPA receptor responses, is activity dependent. Blocking the overall activity of rat organotypic hippocampal slices either with TTX or group 1 metabotropic glutamate receptor (mGluR) antagonists, especially mGluR5 antagonist, prevented the AMPAR transmission increase caused by PSD-95 overexpression (Zhang and Lisman, 2012). At the same time, it is known that group 1 mGluRs modulate the NMDA receptor function through pathways changing the phosphorylation state of GluN2A and B subunits (Rojas and Dingledine, 2013). A member of the group 1 mGluRs, mGluR5, has been shown to activate PKC, which in turn activates Src kinase, resulting in the phosphorylation of GluN2B subunit in hippocampal neurons (MacDonald et al., 2007). To understand, whether the effects of the CDK5 phosphorylation mutants of PSD-95 on AMPAR and NMDAR transmission are activity-dependent, I blocked the overall activity of the slices with $1 \mu \mathrm{M}$ TTX or treated the slices with $25 \mu \mathrm{M}$ MPEP, a specific mGluR5 blocker, for two days before the recordings.

Blockade of the network activity by TTX did cause a slight but not significant increase in AMPAR responses of the non-phosphorylatable mutant expressing cells in comparison to the control cells. NMDAR responses were not any different than the controls (AMPAR, uninfected: $-69.2 \pm 17.7$ pA, infected: $-99.7 \pm 20.7$ pA, p=0.06; NMDAR, uninfected: $45.5 \pm 12.8 \mathrm{pA}$, infected: $48.0 \pm 8.9 \mathrm{pA}, \mathrm{p}=0.77)$ (Figure 24A). Interestingly, AMPAR responses of phospho-mimicking mutant expressing cells were enhanced even further, when activity in the slices was blocked. Without the blockade there was a significant $45 \%$ increase in AMPAR responses, whereas the blockade resulted in a four-fold increase. NMDAR transmission was again significantly higher in the phospho-mimicking mutant expressing cells and this enhancement was not particularly different when the activity was blocked or not (AMPAR, uninfected: -41.3 \pm 4.5 pA, infected: $-168.4 \pm 29.5$ pA, p<0.002; NMDAR, uninfected: $49.8 \pm 11.6$ pA, infected: $80.9 \pm 15.3 \mathrm{pA}, \mathrm{p}<0.05$ ) (Figure 24B). Thus, activity blockade revealed a sensitivity of the phospho-mimicking mutant specifically in AMPA receptor transmission. 


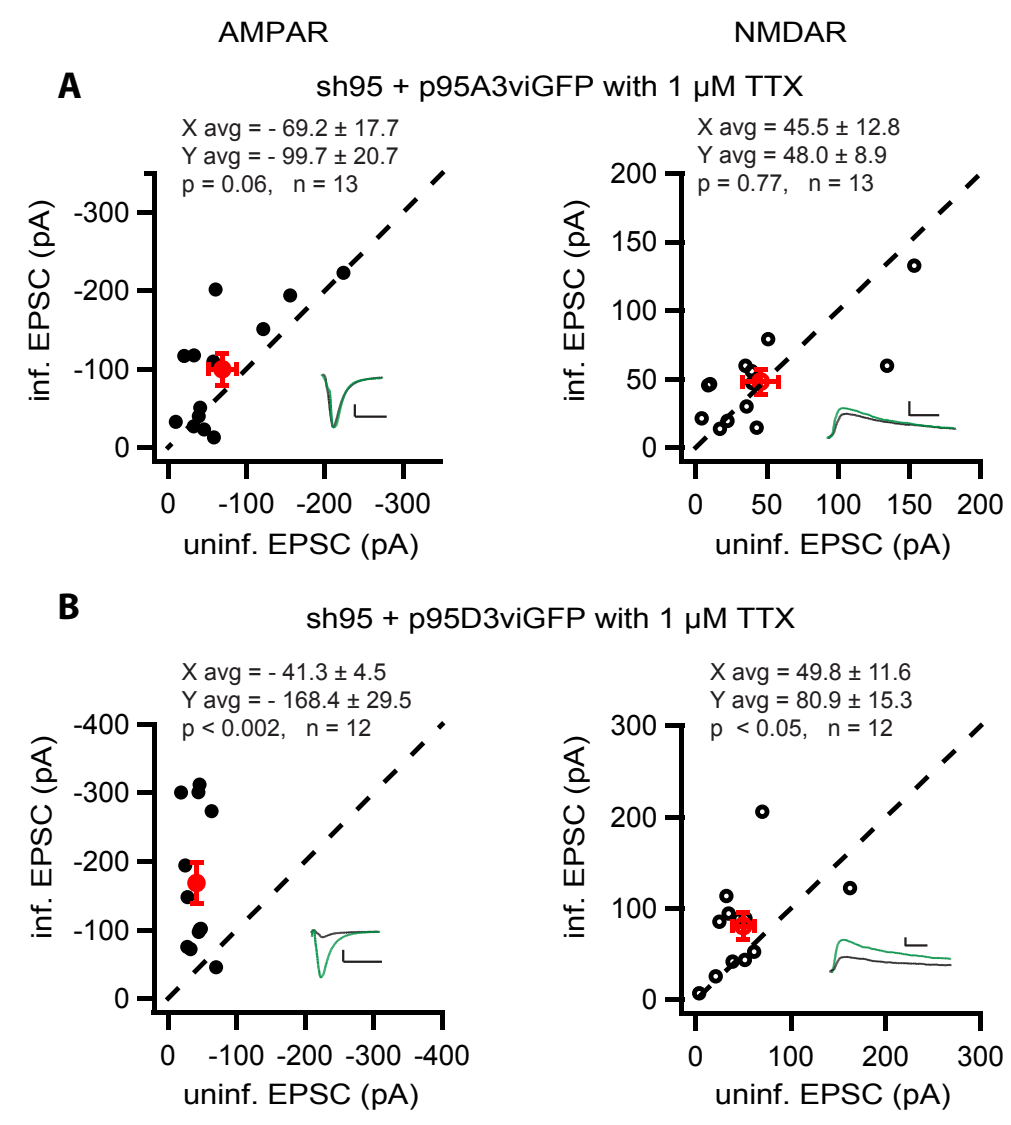

Figure 24: Blockade of the slice activity for two days by TTX treatment enhances the effect of phospho-mimicking mutant of PSD-95 on AMPAR transmission in CA1 region neurons.

(A) Activity blockade did not cause a significant difference in AMPAR ( $\mathrm{p}=0.06)$ and NMDAR transmission $(\mathrm{p}=077)$ between the non-phosphorylatable mutant expressing and control cells.

(B) Activity blockade caused a four-fold increase in AMPAR transmission when phosphomimicking mutant was expressed in the cells $(\mathrm{p}<0.002)$. NMDAR transmission was still significantly higher than the control cells $(\mathrm{p}<0.05)$.

(The scale bars correspond to $50 \mathrm{pA}$ and $25 \mathrm{~ms}$. Green traces indicate the infected and the black traces indicate the control cell responses. The red marker corresponds to the average of all data points on the plot.)

On the other hand, blocking the mGluR5 activity with $25 \mu \mathrm{M}$ MPEP, a specific mGluR5 inhibitor, resulted in a significant increase in AMPAR responses of nonphosphorylatable mutant expressing cells, whereas NMDAR transmission was no different than the control cells (AMPAR, uninfected: $-106.8 \pm 24.1 \mathrm{pA}$, infected: -158.7 \pm 25.9 pA, $<<0.05$; NMDAR, uninfected: $48.1 \pm 9.4$ pA, infected: $65.4 \pm 12.9 \mathrm{pA}$, $\mathrm{p}=0.08$ ) (Figure 25A). In contrast, MPEP application eliminated the significant increase in NMDAR transmission normally detected with the phospho-mimicking mutant 
expressing cells. However, it did not effect the AMPAR transmission of the infected cells, which was still significantly higher than the control cells (AMPAR, uninfected: $37.2 \pm 4.8$ pA, infected: $-44.2 \pm 16.2$ pA, p<0.05; NMDAR, uninfected: $79.3 \pm 12.6$ pA, infected: $64.3 \pm 2.7 \mathrm{pA}, \mathrm{p}=0.32$ ) (Figure 25B). These results demonstrate that mGluR5 activity affects the function of the mutants differently regarding AMPAR and NMDAR transmission.

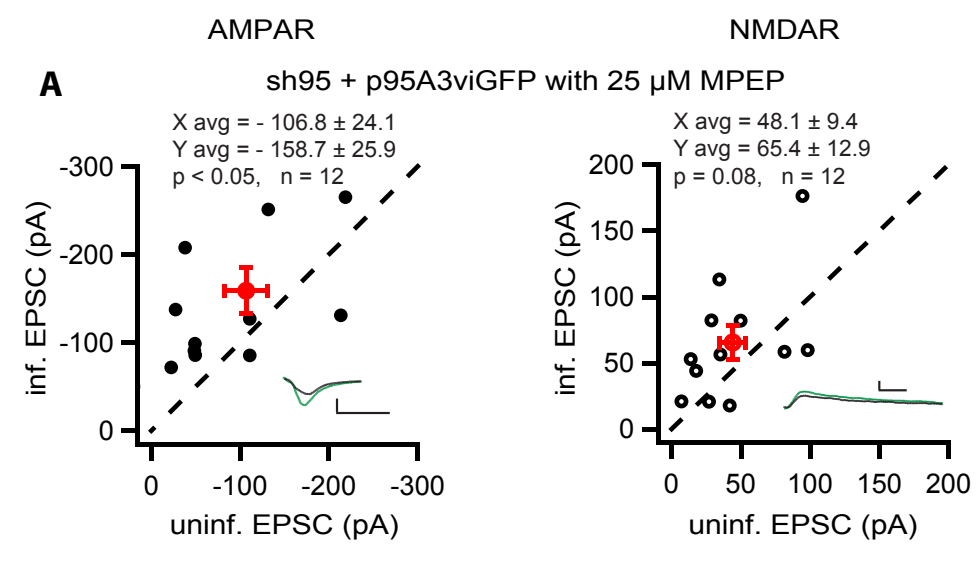

B sh95 + p95D3viGFP with $25 \mu \mathrm{M}$ MPEP
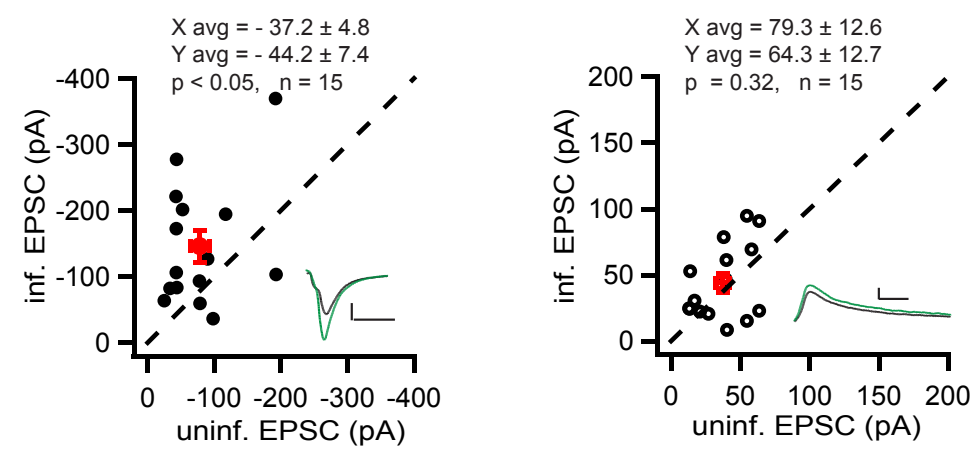

Figure 25: Blockade of mGluR5 activity in the slices for two days enhanced the AMPAR transmission of non-phosphorylatable mutant while eliminating the increase in NMDAR responses normally observed with phospho-mimicking mutant in CA1 region neurons.

(A) mGluR5 blockade by MPEP resulted in a significant increase in AMPAR $(\mathrm{p}<0.05)$ but not in NMDAR transmission of non-phosphorylatable mutant expressing cells $(p=0.08)$.

(B) mGluR5 blockade by MPEP eliminated the significant increase in NMDAR transmission $(p=0.32)$, which is normally observed with phospho-mimicking mutant expression. AMPAR transmission was still significantly higher in infected cells $(\mathrm{p}<0.05)$ in comparison to the controls, which seems to be independent of mGluR5 activity.

(The scale bars correspond to $50 \mathrm{pA}$ and $25 \mathrm{~ms}$. Green traces indicate the infected and the black traces indicate the control cell responses. The red marker corresponds to the average of all data points on the plot.) 


\subsubsection{Pre- and postsynaptic properties of the CDK5 phosphorylation mutants of PSD-95}

Besides stimulating the Schaffer collaterals and recording evoked responses, I wanted to test the integrated input received by neurons expressing either of the PSD-95 mutants. This approach would demonstrate whether the effects of the mutants are pathwayspecific or valid for all the synapses. For this purpose, I recorded miniature EPSCs from the CA1 region rat neurons infected with the phosphorylation mutants of PSD-95. Control and infected cells in each group were recorded from the same slices in the presence of TTX. There was a significant decrease in the mEPSC amplitudes of phospho-mimicking mutant expressing cells but the mEPSC frequency was not different than the control cells (D3viG mean \pm SEM, amplitude, control: $20.74 \pm 0.79$ pA, infected: $17.66 \pm 0.69 \mathrm{pA}$; frequency, control: $1.86 \pm 1.26 \mathrm{~s}$, infected: $1.96 \pm 0.26$ $\mathrm{s}$; Kolmogorov-Smirnov test, amplitude: $\mathrm{p}<0.05$, frequency: $\mathrm{p}=1$ ) (Figure 26B). Only a small but non-significant increase was observed in the amplitude of mEPSCs with the non-phosphorylatable mutant and the frequency of the events was not altered between the infected and control cells (A3viG mean \pm SEM, amplitude, control: $20.83 \pm 0.70$ pA, infected: $23.28 \pm 1.12 \mathrm{pA}$; frequency, control: $2.42 \pm 0.24 \mathrm{~s}$, infected: $2.32 \pm 0.24$ $\mathrm{s}$; Kolmogorov-Smirnov test, amplitude: $\mathrm{p}=0.19$, frequency: $\mathrm{p}=0.47$ ) (Figure 26A). 

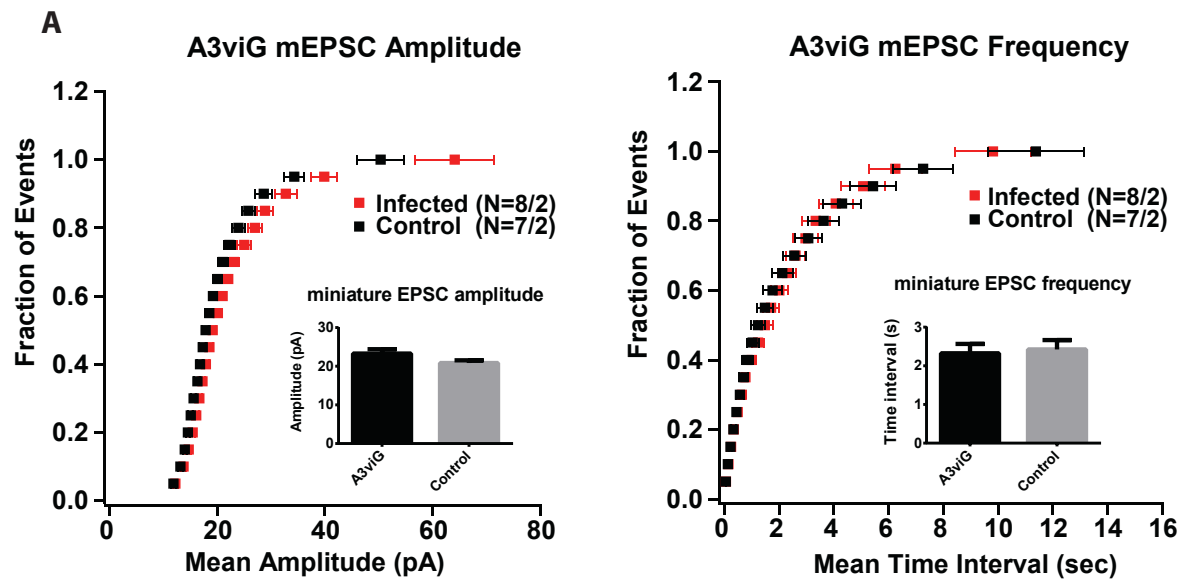

B

D3viG mEPSC Amplitude
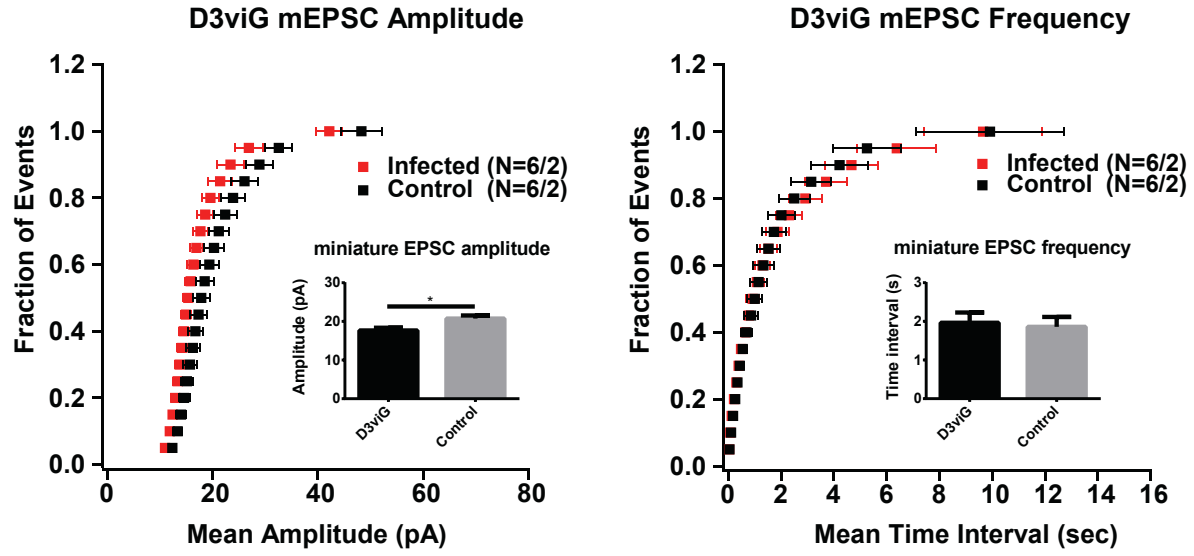

Figure 26: CDK5 phosphorylation mutants of PSD-95 do not alter the frequency of miniature EPSCs in CA1 region neurons.

Cumulative distribution plot represents the amplitude and frequency distribution of infected and control cell mEPSCs.

(A) There was only a slight but non-significant increase in the mEPSC amplitude of nonphosphorylatable mutant expressing cells. The frequency was not changed between the control and the infected cells. (A3viG mean \pm SEM, amplitude, control: $20.83 \pm 0.70$, infected: $23.28 \pm$ 1.12; frequency, control: $2.42 \pm 0.24$, infected: $2.32 \pm 0.24$; Kolmogorov-Smirnov test, amplitude: $\mathrm{p}=0.19$, frequency: $\mathrm{p}=0.47$ )

(B) There was a significant decrease in the mEPSC amplitude of phospho-mimicking mutant expressing cells. The frequency of mEPSC events was not altered between the control and mutant expressing cells. (D3viG mean \pm SEM, amplitude, control: $20.74 \pm 0.79$, infected: 17.66 \pm 0.69 ; frequency, control: $1.86 \pm 1.26$, infected: $1.96 \pm 0.26$; Kolmogorov-Smirnov test, amplitude: $p<0.05$, frequency: $p=1$ )

(Red data points represent the infected and black data points represent the control cell values. For statistics Kolmogorov-Smirnov test was used. $\mathrm{N}=$ number of cells / number of cultures)

This result was unexpected, as the phospho-mimicking mutant demonstrated an increase in the AMPAR transmission in evoked events, however showed a decrease in the amplitude of mEPSCs. At the same time, having no change in the frequency of 
mEPSCs with both of the mutants hinted that the presynaptic properties of the mutant expressing neurons were not affected. In order to confirm that the presynaptic release property of the neurons were not altered I measured the paired-pulse ratio of evoked AMPAR responses of infected and control cells in a dual-patch configuration. The responses were triggered with $100 \mathrm{~ms}$ interval. Both control and mutant expressing cells showed an increase of 1.4 fold at the second pulse and there was no significant difference between the ratios of infected and control cells (D3viG, control: $1.47 \pm 0.06$, infected: $1.44 \pm 0.04 ; \mathrm{A} 3 \mathrm{viG}$, control: $1.38 \pm 0.08$, infected: $1.33 \pm 0.07$ ) (Figure 27).
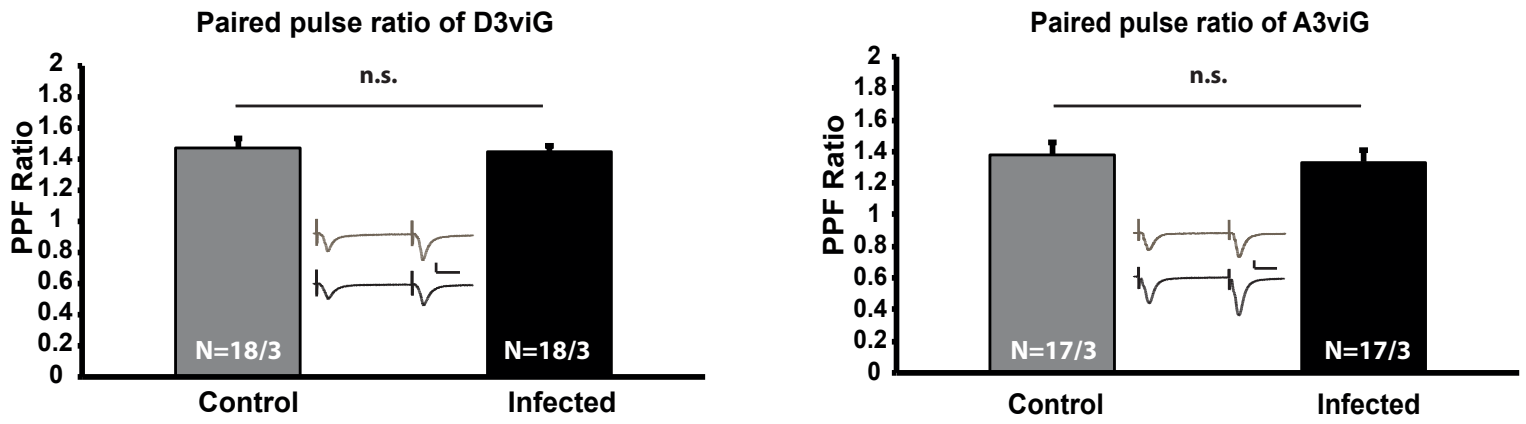

Figure 27: No difference was observed between the paired-pulse ratio of mutant expressing and control cells in CA1 region neurons.

Two stimulations were applied with $100 \mathrm{~ms}$ time interval at $-60 \mathrm{mV}$. Both control and CDK5 phosphorylation mutants of PSD-95 expressing cells showed a facilitation of $40 \%$. Bar graphs represent the mean \pm SEM values. (D3viG, control: $1.47 \pm 0.06$, infected: $1.44 \pm 0.04$; A3viG, control: $1.38 \pm 0.08$, infected: $1.33 \pm 0.07)$ Tested by Students t-test.

(The scale bars correspond to $50 \mathrm{pA}$ and $25 \mathrm{~ms}$. Grey sample traces represent the control, black sample traces represent the infected cell responses. For statistical analysis Students t-test was used.

$\mathrm{N}=$ number of cells / number of cultures)

Besides the presynaptic properties, Morabito et al., 2004 showed that the blockade of CDK5 activity via a dominant negative construct, or the expression of the nonphosphorylatable mutant of PSD-95 would form bigger PSD-95 clusters at hippocampal synapses (Morabito et al., 2004). With respect to this result, one can speculate that the AMPAR responses of the non-phosphorylatable mutant expressing cells would be higher than the controls. Interestingly, this was not the case for the evoked responses. This observation might be due to an effect on extrasynaptic AMPA receptor population, which will not be targeted by the synaptic stimulation. To examine whether the non-phosphorylatable mutant increases the extra-synaptic AMPA receptor 
pool, I performed a glutamate spill-over experiment by using a competitive glutamate transporter blocker, $100 \mu \mathrm{M}$ DL-TBOA. The responses from the control and nonphosphorylatable mutant expressing cells were recorded again in the presence of DLTBOA. Application of DL-TBOA did not reveal a difference between the infected and control cells (AMPAR, uninfected: $-55.1 \pm 10$ pA, infected: $-44.4 \pm 7.2 \mathrm{pA}, \mathrm{p}=0.25$; NMDAR, uninfected: $47.4 \pm 12.1 \mathrm{pA}$, infected: $35.1 \pm 5.9 \mathrm{pA}, \mathrm{p}=0.17$ ) (Figure 28), suggesting that the non-phosphorylatable mutant does not regulate the extra-synaptic receptor pool.

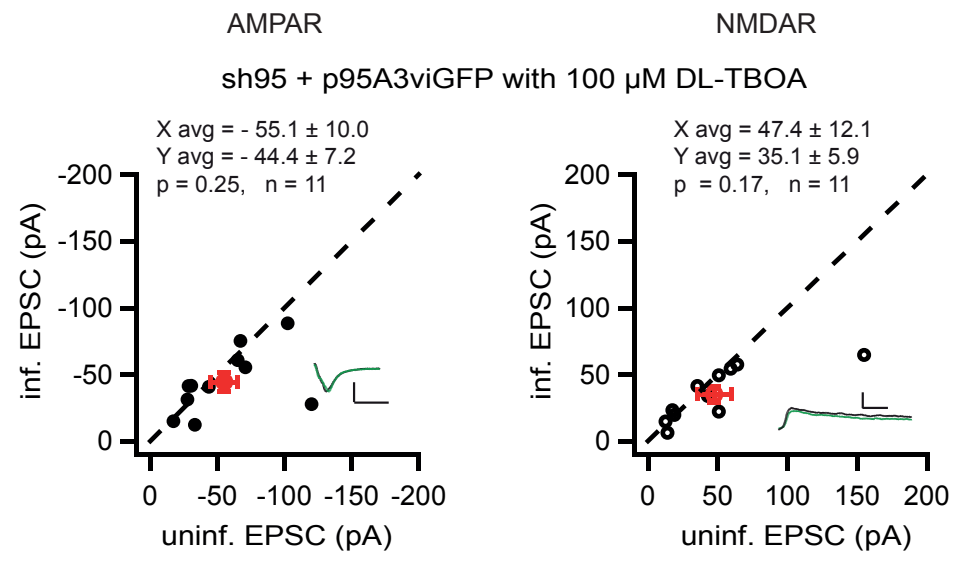

Figure 28: Non-phosphorylatable mutant of PSD-95 does not affect the extra-synaptic AMPA receptor pool in $\mathrm{CA} 1$ region neurons.

Application of DL-TBOA, thus glutamate spill-over did not cause a change in AMPAR and NMDAR transmission between the non-phosphorylatable mutant expressing and control cells.

(The scale bars correspond to $50 \mathrm{pA}$ and $25 \mathrm{~ms}$. Green traces indicate the infected and the black traces indicate the control cell responses. The red marker corresponds to the average of all data points on the plot.)

\subsection{The role of CDK5 phosphorylation mutants of PSD- 95 in the regulation of developmental NMDA receptor subunit switch in mouse visual cortex}

The composition of NMDA receptors has been shown to shift from GluN2B-containing NMDARs to dominantly GluN2A-containing receptors over the course of development (Bellone and Nicoll, 2007). This change takes place in an activity-dependent manner within the first 2-3 weeks of the postnatal period in different brain areas, including hippocampus and various cortical regions (Dumas, 2005). In the visual cortex, the switch is precisely triggered by the first visual stimulus upon eye opening (Philpot et al., 
2001) This makes it a suitable system to investigate the NMDA receptor subunit switch. Although the involvement of PSD-95 in this process has been suggested by several studies (Losi et al., 2003a; Yoshii et al., 2003; Liu et al., 2004c; Funahashi et al., 2013), the exact mechanism mediating this switch is not known. Recently, it has been shown that the interaction between PSD-95, CDK5 and Src kinase is involved in the regulation of GluN2B-containing NMDA receptor surface expression. In order to understand whether this can be a mechanism to regulate the NMDAR subunit switch, I decided to test the components of this pathway. At first, I needed to confirm that the developmental NMDAR subunit switch takes place in the mouse visual cortex layer $2 / 3$ neurons upon eye opening. To accomplish this, I recorded the NMDAR responses of layer $2 / 3$ neurons from the animals with the ages corresponding to before (P10-12) and after eye opening (P14-20). The recordings were performed in the presence of $5 \mu \mathrm{M}$ NBQX and at $+40 \mathrm{mV}$ to isolate pure NMDAR responses. After the baseline was established, $5 \mu \mathrm{M}$ ifenprodil was washed in for 25 minutes to check the contribution of GluN2B-containing receptors in NMDAR responses. The results clearly showed that upon eye opening, GluN2B-containing receptors contribution to NMDA receptor responses was reduced. Ifenprodil wash-in in younger animals resulted in a $50 \%$ blockade of the responses, whereas the effect in animals after eye opening was only 20\% (Last 4 minutes of the recordings were compared. Students t-test, AEO: $0.83 \pm$ 0.04; BEO: $0.51 \pm 0.02, \mathrm{p}<0.0001$ ) (Figure 29). 
NMDAR Subunit Composition Before vs After Eye Opening
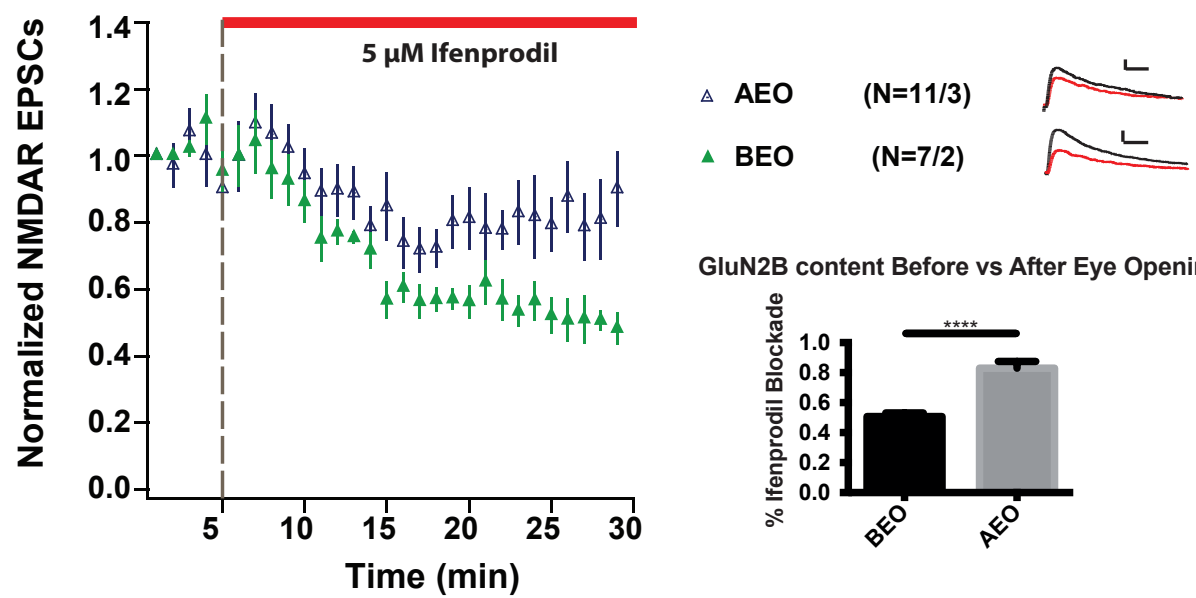

GluN2B content Before vs After Eye Opening

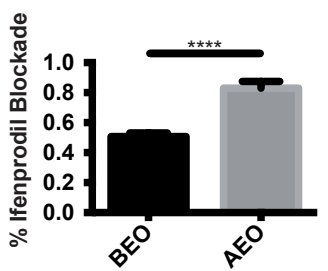

Figure 29: The contribution of GluN2B-containing NMDA receptors to NMDAR responses is reduced after eye opening in visual cortex later $2 / 3$ neurons.

Application of ifenprodil blocked $50 \%$ of the NMDAR responses before the eye opening period. However, this blockade was only $20 \%$ after the eye opening. The responses were normalized to the initial NMDAR amplitude. Bar graphs represent the mean \pm SEM of binned responses of the last 4 minutes of the recordings (Students t-test, AEO: $0.83 \pm 0.04$; BEO: $0.51 \pm 0.02, \mathrm{p}<0.0001$ ).

(The scale bars correspond to $50 \mathrm{pA}$ and $25 \mathrm{~ms}$. Black sample traces indicate NMDAR responses before and red sample traces after ifenprodil wash-in. Green data points indicate responses of neurons corresponding to before eye opening (BEO) period and the blue data points indicate the responses of neurons after eye opening (AEO). The gray dashed line indicates the time point where the ifenprodil wash-in started. $\mathrm{N}=$ number of cells / number of animals)

After confirming that the NMDA receptor subunit switch indeed takes place in visual cortex layer 2/3 neurons upon first visual stimulus, I examined the effect of PSD95 absence on subunit switch by recording from the slices of PSD-95 knockout animals after the eye opening. The recordings demonstrated that the lack of PSD-95 prevented the switch from taking place, since the knockout animals exhibited a blockade rate of $50 \%$, even though eye opening took place (Last four minutes of the recordings were compared. one-way ANOVA post-hoc Tukey test, $\mathrm{F}(2,90)=26.20, \mathrm{p}<0.0001$; AEO: $0.83 \pm 0.04$; BEO: $0.51 \pm 0.02$; P95 KO AEO: $0.44 \pm 0.04$ ) (Figure 30). 


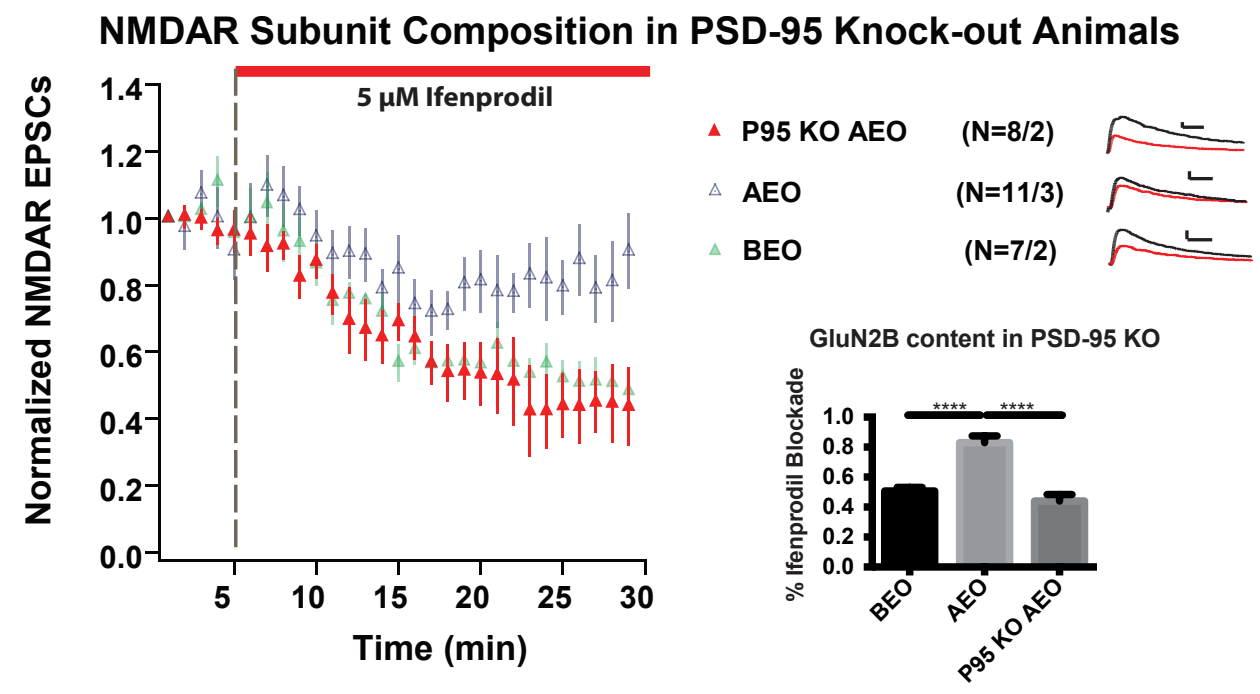

Figure 30: PSD-95 knockout animals are deficient in establishing the NMDA receptor subunit switch upon eye opening in mouse visual cortex layer $2 / 3$ neurons.

Application of ifenprodil blocked 50\% of the NMDAR responses of the neurons from PSD-95 KO slices, eventhough the eye opening took place. The responses were normalized to the initial NMDAR amplitude. Bar graphs represent the mean \pm SEM of binned responses from the last four minutes of the recordings (one-way ANOVA post-hoc Tukey test, $\mathrm{F}(2,90)=26.20$, p $<0.0001$; AEO: $0.83 \pm 0.04$; BEO: $0.51 \pm$ 0.02; P95 KO AEO: $0.44 \pm 0.04)$.

(The scale bars correspond to $50 \mathrm{pA}$ and $25 \mathrm{~ms}$. Black traces indicate NMDAR responses before and red traces after ifenprodil wash-in. Green data points indicate responses of neurons corresponding to before eye opening (BEO) period and the blue data points indicate the responses of neurons after eye opening (AEO). Red data points indicate the responses of PSD-95 KO neurons after eye opening (P95 KO AEO). The gray dashed line indicates the time point where the ifenprodil wash-in started. $\mathrm{N}=$ number of cells / number of animals)

Although the recordings from the knockout animals imply that PSD-95 is involved in the NMDAR subunit switch, it is possible that knockout systems generate compensatory mechanisms and network changes, which can interfere with the results. In order to exclude these factors and dissect the specific role of PSD-95 in the process, I injected lentivirus carrying an shRNA sequence against PSD-95 into the visual cortex of newborn mice. After the viral expression was established, the animals were sacrificed at an age $(\mathrm{P}=14-20)$ after eye opening has taken place. Very convincingly, the single cell knock-down of PSD-95 resulted in a phenotype similar to knockout animals and demonstrated a 50\% blockade of NMDAR responses upon GluN2B blockade (Last four minutes of the recordings were compared. one-way ANOVA posthoc Tukey test, $\mathrm{F}(3,128)=29.73, \mathrm{p}<0.0001$; AEO: $0.83 \pm 0.04$; BEO: $0.51 \pm 0.02$; P95 
KO AEO: $0.44 \pm 0.04$; PSD-95 in vivo KD: $0.42 \pm 0.03$ ) (Figure 31). This result strongly demonstrates the importance of PSD-95 in NMDAR subunit switch in a single cell level.

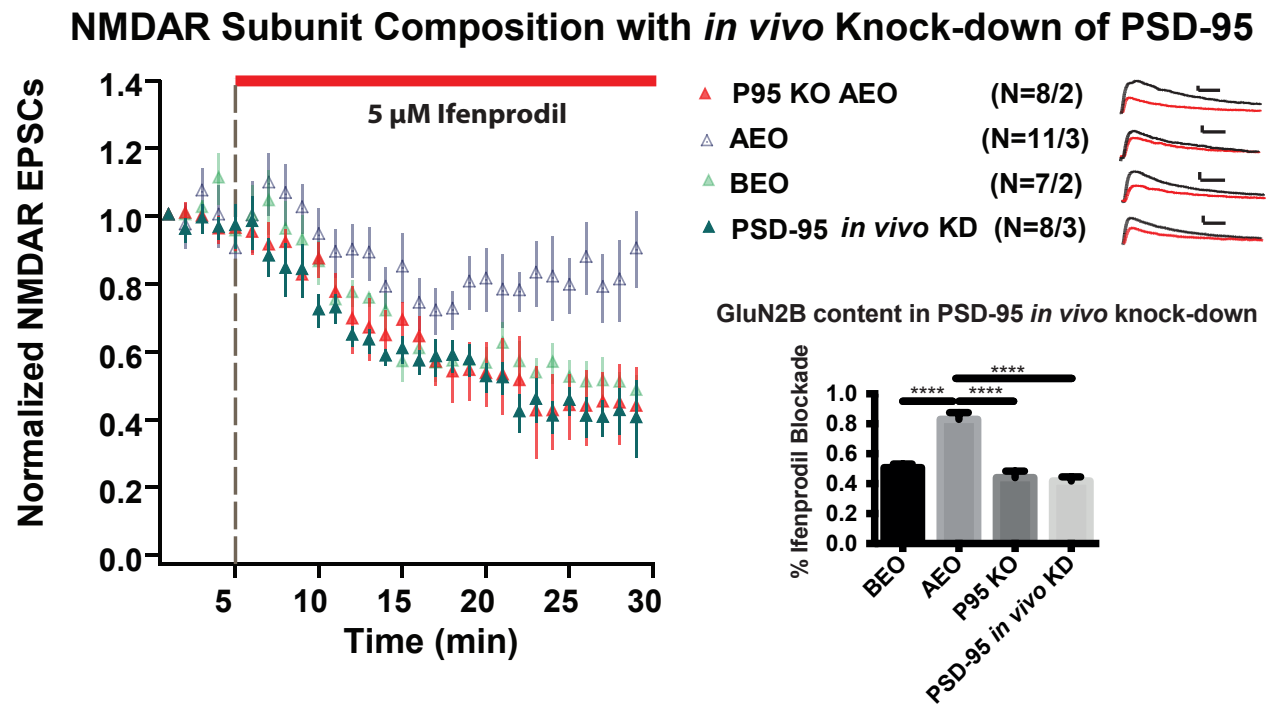

Figure 31: PSD-95 is involved in the NMDAR subunit switch in mouse visual cortex.

Injection of lentivirus carrying shRNA against PSD-95 prevented the switch in single cells, demonstrating that PSD-95 is crucial for the developmental NMDAR subunit switch. The responses were normalized to the initial NMDAR amplitude. Bar graphs represent the mean \pm SEM of binned responses from the last four minutes of the recordings (one-way ANOVA post-hoc Tukey test, F(3,128) = 29.73, $\mathrm{p}<0.0001$; AEO: $0.83 \pm 0.04$; BEO: $0.51 \pm 0.02$; P95 KO AEO: $0.44 \pm 0.04$; PSD-95 in vivo KD: $0.42 \pm$ $0.03)$.

(The scale bars correspond to $50 \mathrm{pA}$ and $25 \mathrm{~ms}$. Black traces indicate NMDAR responses before and red traces after ifenprodil wash-in. Green data points indicate responses of neurons corresponding to before eye opening (BEO) period and the blue data points indicate the responses of neurons after eye opening (AEO). Red data points indicate the responses of PSD-95 KO neurons after eye opening (P95 KO AEO). Dark green data points represent the responses of neurons injected with PSD-95 shRNA, after the eye opening took place (PSD-95 in vivo KD). The gray dashed line indicates the time point where the ifenprodil wash-in started. $\mathrm{N}=$ number of cells / number of animals)

After validating the involvement of PSD-95 in NMDAR subunit switch, the next step was to test the involvement of PSD-95, CDK5 and Src kinase interaction in this switch. To address this, I used the CDK5 phosphorylation mutants of PSD-95. First, I injected newborn animals with wild-type PSD-95 replacement construct. Recording from the animals injected with wild-type replacement after the eye opening showed a blockade of $30 \%$ in NMDAR transmission upon ifenprodil wash-in, which was $10 \%$ less than the blockade rate observed in wild-type animals after the eye opening (WtviG AEO: $0.67 \pm 0.02$, AEO: $0.83 \pm 0.04$, Students t-test, $\mathrm{p}<0.05)$. Next, I injected animals 
with the virus carrying non-phosphorylatable and phospho-mimicking mutants of PSD95 and again measured the blockade rate of NMDAR responses after the eye opening. Interestingly, non-phosphorylatable mutant expressing cells behaved in the same way as the wild-type replacement, whereas phospho-mimicking mutant expressing cells were deficient in NMDAR subunit switch. The blockade rate observed with phosphomimicking mutant was almost $60 \%$ similar to before eye opening period of wild-type animals (Last four minutes of the recordings were compared. one-way ANOVA posthoc Tukey test, $\mathrm{F}(2,68)=25.21, \mathrm{p}<0.0001$; WtviG AEO: $0.67 \pm 0.02$; A3viG AEO: $0.70 \pm 0.03 ;$ D3viG AEO: $0.40 \pm 0.03$ ) (Figure 32).

The Effect of CDK5 Mutants of PSD-95 on NMDAR Subunit Composition
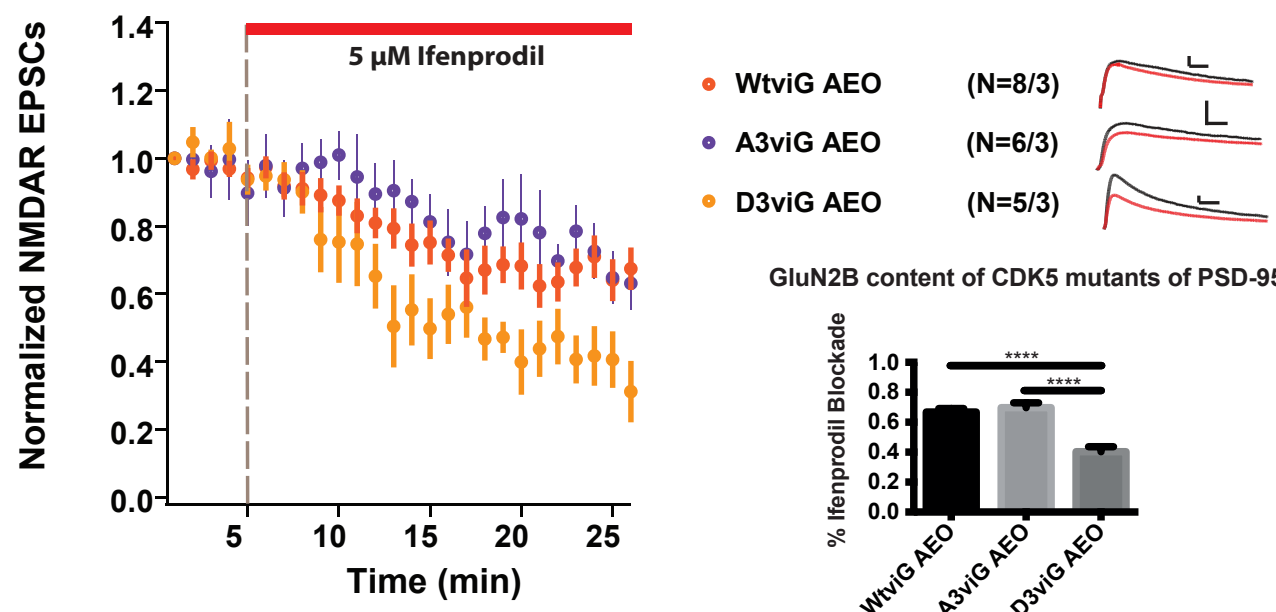

GluN2B content of CDK5 mutants of PSD-95

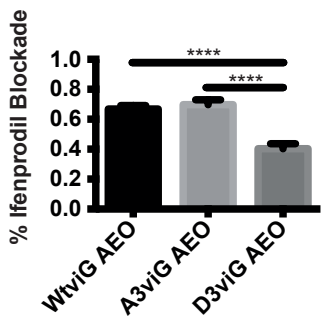

Figure 32: Phospho-mimicking mutant of PSD-95 prevents the NMDAR subunit switch upon eye opening.

Injection of lentivirus carrying wild-type replacement construct of PSD-95 resulting in a 30\% blockade of the NMDAR responses after ifenprodil wash-in. Non-phosphorylatable mutant expressing neurons demonstrated a similar blockade pattern, whereas phosphor-mimicking mutant expressing cells showed a $60 \%$ reduction in NMDAR responses after ifenprodil wash-in. The responses were normalized to the initial NMDAR amplitude. Bar graphs represent the mean \pm SEM of binned responses from the last four minutes of the recordings (one-way ANOVA post-hoc Tukey test, $F(2,68)=25.21, \mathrm{p}<0.0001$; WtviG AEO: $0.67 \pm 0.02$; A3viG AEO: $0.70 \pm 0.03$; D3viG AEO: $0.40 \pm 0.03$ ).

(The scale bars correspond to $50 \mathrm{pA}$ and $25 \mathrm{~ms}$. Black traces indicate NMDAR responses before and red traces after ifenprodil wash-in. Orange data points indicate responses of neurons expressing wild-type replacement of PSD-95, after the eye opening took place (WtviG AEO). Purple data points represent non-phosphorylatable mutant expressing cell responses (A3viG AEO) and yellow points phosphomimicking mutant expressing cell responses (D3viG), after the eye opening. The gray dashed line indicates the time point where the ifenprodil wash-in started. $\mathrm{N}=$ number of cells / number of animals) 
The results collected from the mutant recordings demonstrate that the NMDA receptor subunit switch might be regulated depending on the CDK5 phosphorylation state of PSD-95.

\subsection{Minimal PSD-95 to mediate the basal synaptic transmission and a molecular interplay between the MAGUK family members}

It has been shown by $\mathrm{Xu}$ et al., 2008 that the expression of a PSD-95 deletion mutant with only the first two PDZ domains was not functional in CA1 region neurons of rat hippocampus, when endogenous PSD-95 was knocked-down via shRNA mediated silencing. In addition, the expression of PSD-95 lacking SH3 and GK domains resulted in a similar phenotype, pointing out the importance of SH3 and GK domains in the regulation of hippocampal basal synaptic transmission. In order to investigate the role of PDZ3, SH3 and GK domains in this regulation, a set of experiments using different domain mutants of PSD-95 was performed in collaboration with Dr. Stéphanie Bonnet (former member of Molecular Neurobiology lab, ENI, Goettingen, Germany) and Dr. Yanling Liu (Molecular Neurobiology lab, ENI, Goettingen, Germany).

First, we recorded the AMPAR and NMDAR responses of neurons transduced with viruses expressing shRNA construct against endogenous PSD-95 (sh95), wild-type PSD-95 replacement (sh95 + p95::GFP) and PDZ1/2 (sh95 + PDZ1/2::GFP) replacement to confirm the previously published data. As shown before, knock-down of endogenous PSD-95 by shRNA mediated silencing resulted in a 50\% decrease in AMPAR responses (uninfected: $-92.7 \pm 9.6$ pA, infected: $-54.4 \pm 7.6 \mathrm{pA}, \mathrm{p}<0.001$ ) without a change in NMDAR responses (uninfected: $60.0 \pm 6.7 \mathrm{pA}$, infected: $57.6 \pm 6.9$ $\mathrm{pA}, \mathrm{p}=0.59)$ (Figure 33A). Wild-type PSD-95 replacement demonstrated a significant increase in AMPAR mediated responses (uninfected: - $43.0 \pm 9.5 \mathrm{pA}$, infected: $-83.0 \pm$ $13.5 \mathrm{pA}, \mathrm{p}<0.005$ ) with a slight increase in NMDAR responses (uninfected: $41.4 \pm 7.5$ pA, infected: $58.0 \pm 8.0$ pA, p < 0.05) (Figure 33B). Lastly, replacement of endogenous PSD-95 with PDZ1/2::GFP construct showed significant decrease in AMPAR responses (uninfected: $-47.1 \pm 7.1 \mathrm{pA}$, infected: $-30.3 \pm 5.6 \mathrm{pA}, \mathrm{p}<0.001$ ) with no 
A

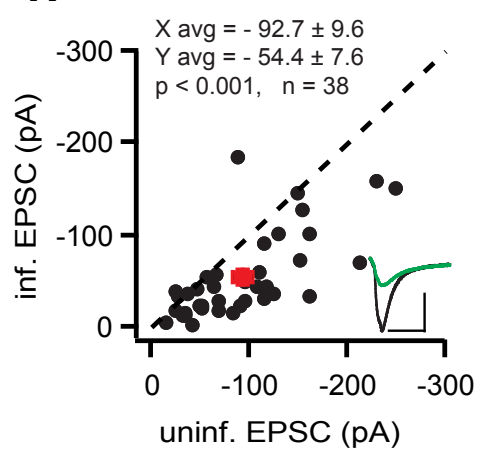

sh95

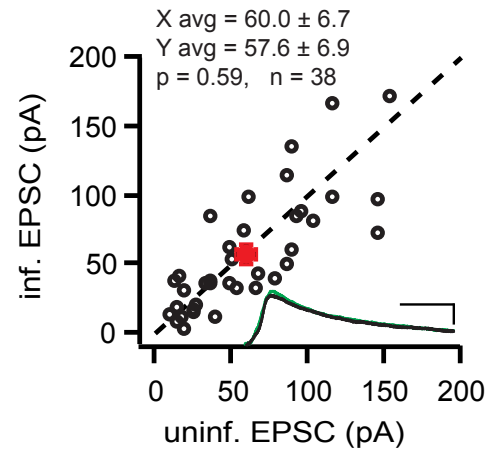

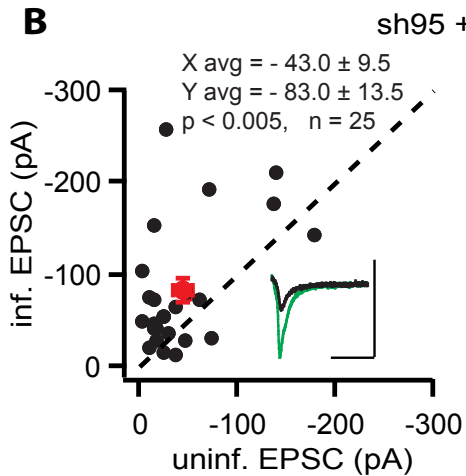

h95 + PSD-95::GFP
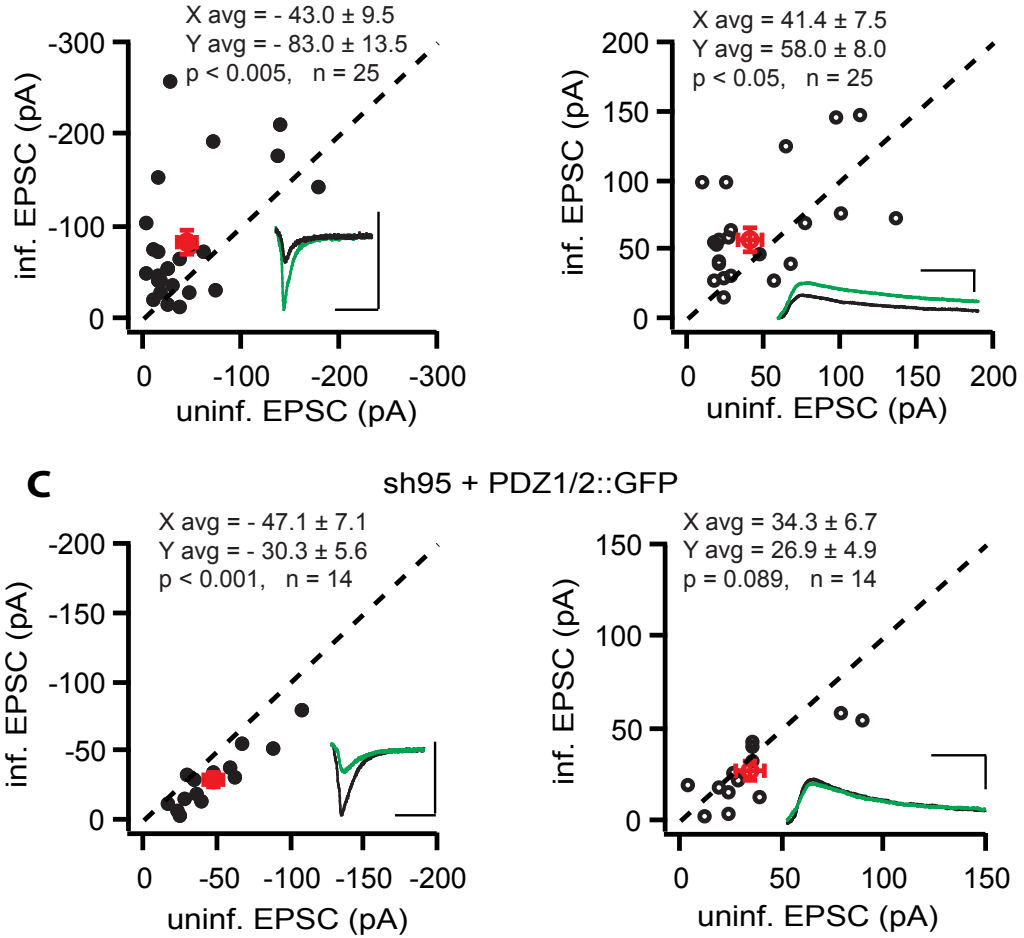

Figure 33: PSD-95 replacement increases AMPAR responses while knock-down of endogenous PSD-95 or replacement of it by PDZ1 $\backslash 2$ ::GFP cause a significant decrease in AMPAR transmission in $\mathrm{CA1} 1$ region rat hippocampal neurons.

(A) Knock-down of endogenous PSD-95 significantly reduced AMPAR mediated responses in infected cells $(p<0.001)$ while not changing NMDAR mediated transmission $(p=0.59)$ (Performed by Stéphanie Bonnet).

(B) Replacement of endogenous PSD-95 by wild-type PSD-95 significantly increased AMPAR $(\mathrm{p}<0.005)$ and NMDAR responses $(\mathrm{p}<0.05)$ of infected cells (Performed by Stéphanie Bonnet).

(C) Replacement of endogenous PSD-95 by PDZ1/2::GFP mutant of PSD-95 depressed AMPAR mediated responses $(p<0.001)$ without altering the NMDAR transmission $(p=0.089)$ (Performed by Stéphanie Bonnet).

(The scale bars correspond to $50 \mathrm{pA}$ and $25 \mathrm{~ms}$. Green traces indicate the infected and the black traces indicate the control cell responses. The red marker corresponds to the average of all data points on the plot.) 
change in NMDAR responses (uninfected: $34.3 \pm 6.7$, infected: $26.9 \pm 4.9, \mathrm{p}=0.089$ ) (Figure 33C).

The next step was to separately test the involvement of PDZ3, SH3 and GK domains in the regulation of basal synaptic transmission in rat hippocampus. With this aim, PSD-95 mutants lacking either of the domains were expressed in CA1 region neurons and AMPAR and NMDAR responses were recorded simultaneously from infected and control cells. Replacing endogenous PSD-95 with a mutant form lacking the PDZ3 domain (sh95 + PSD-95 $\Delta$ PDZ3::GFP) did not enhance the AMPAR (uninfected: - $93.0 \pm 8.8 \mathrm{pA}$, infected: $-103.4 \pm 9.3 \mathrm{pA}, \mathrm{p}=0.22$ ) and NMDAR responses (uninfected: $70.0 \pm 6.5 \mathrm{pA}$, infected: $66.0 \pm 6.0 \mathrm{pA}, \mathrm{p}=0.55$ ) of the infected cells (Figure 34A). This result indicated that the PSD-95 $\triangle \mathrm{PDZ3}:$ GFP construct does not act in the same way as the full-length PSD-95, as the increase in AMPAR responses observed with full-length PSD-95 replacement was absent. On the other hand, expression of a PSD-95 mutant lacking the SH3 domain (sh95 + PSD-95 $\Delta$ SH3::GFP) demonstrated significant increases both in AMPAR (uninfected: - $33.4 \pm 4.6 \mathrm{pA}$, infected: $-64.9 \pm 8.2 \mathrm{pA}, \mathrm{p}<0.001$ ) and NMDAR transmission (uninfected: $49.5 \pm 7.7$ pA, infected: $66.8 \pm 10.2 \mathrm{pA}, \mathrm{p}<0.005)$ in a similar manner to the full-length PSD-95 replacement (Figure 34B). A third PSD-95 mutant lacking the GK domain (PSD-95 $\Delta G K:: G F P)$ exhibited similar effects on AMPAR and NMDAR transmission as the PSD-95 $\Delta$ PDZ3::GFP mutant replacement. There was no difference between the AMPAR (uninfected: $-55.4 \pm 5.1 \mathrm{pA}$, infected: $-65.8 \pm 6.7 \mathrm{pA}, \mathrm{p}=0.14$ ) and NMDAR transmission (uninfected: $39.9 \pm 3.5 \mathrm{pA}$, infected: $45.6 \pm 4.2 \mathrm{pA}, \mathrm{p}=0.09$ ) of infected and control cells (Figure 34C). The effect of the mutants on AMPAR and NMDAR transmission suggests that the SH3 domain of PSD-95 is dispensable for regulating the basal synaptic transmission in CA1 region neurons. However, at this state of study no conclusion about the roles of PDZ3 and GK domains could be made, as the replacement by mutants lacking either of the domains resulted in a partial rescue of AMPAR responses. 

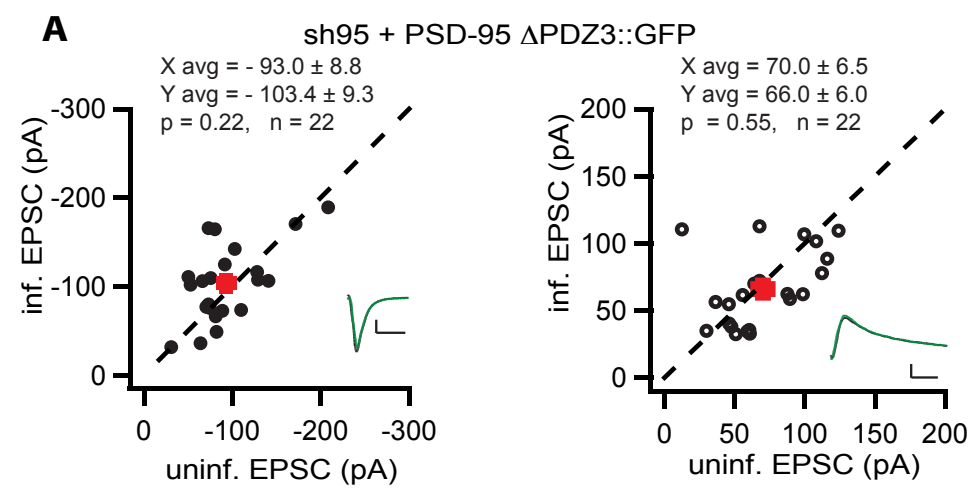

B

sh95 + PSD-95 $\triangle$ SH3::GFP
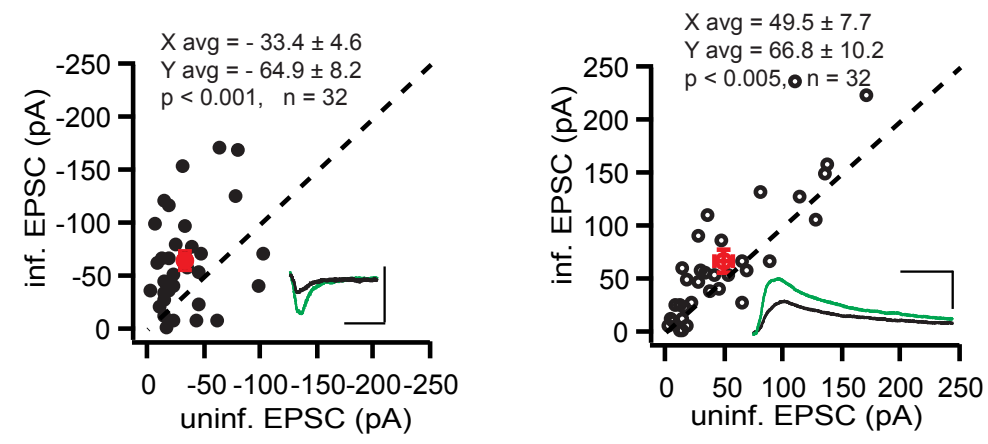

C sh95 + PSD-95 $\Delta$ GK::GFP
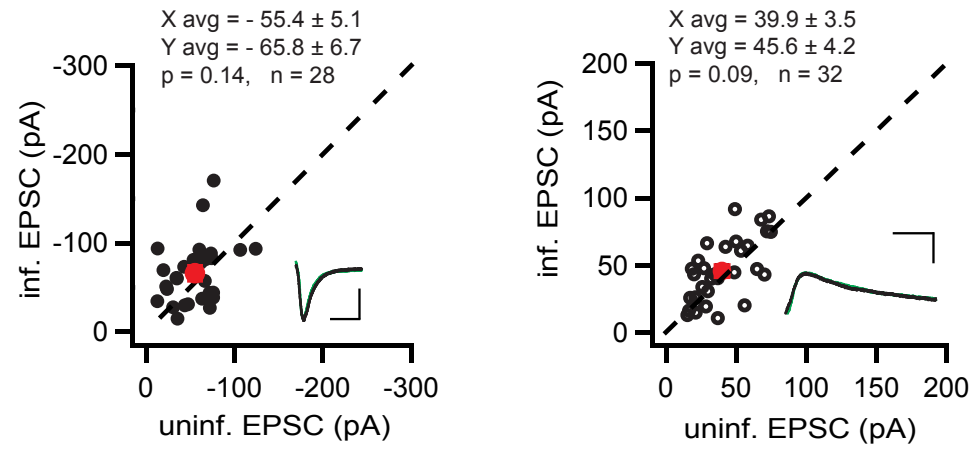

Figure 34: SH3 domain of PSD-95 is dispensable for the regulation of basal synaptic transmission in CA1 region of rat hippocampus, whereas the role of PDZ3 and GK domains is not clear.

(A) Replacement of endogenous PSD-95 by a mutant form lacking PDZ3 domain does not enhance AMPAR $(p=0.22)$ and NMDAR transmission of infected cells $(p=0.55)$ (Performed by Derya Akad)

(B) Replacement of endogenous PSD-95 by a mutant form lacking SH3 domain shows significant enhancement of AMPAR $(\mathrm{p}<0.001)$ and NMDAR responses $(\mathrm{p}<0.005)$, similar to full-length PSD-95 replacement. (Performed by Stéphanie Bonnet)

(C) Replacement of endogenous PSD-95 by a mutant form lacking GK domain shows no difference in AMPAR $(p=0.14)$ and NMDAR transmission $(p=0.09)$ in comparison to control cells. (Performed by Stéphanie Bonnet)

(The scale bars correspond to $50 \mathrm{pA}$ and $25 \mathrm{~ms}$. Green traces indicate the infected and the black traces indicate the control cell responses. The red marker corresponds to the average of all data points on the plot.) 
To check whether the partial rescue observed with the PSD-95 $\Delta$ GK::GFP mutant is due to its functionality, we over-expressed PSD-95 $\Delta \mathrm{GK}:$ GFP in CA1 region rat neurons and compared the results with the full-length PSD-95 overexpression (PSD95::GFP). Overexpression of PSD-95 $\Delta \mathrm{GK}::$ GFP showed a significant increase in infected cell AMPAR responses (uninfected: $-32.2 \pm 5.3 \mathrm{pA}$, infected: $-74.4 \pm 12.0 \mathrm{pA}$, $\mathrm{p}<0.001$ ) similar to PSD-95::GFP overexpression (uninfected: $-68.7 \pm 9.1 \mathrm{pA}$, infected: $-165.9 \pm 19.9 \mathrm{pA}, \mathrm{p}<0.001)$. In both cases, there was no significant change in NMDAR responses between the infected and uninfected cells (PSD-95 $\Delta \mathrm{GK}:: \mathrm{GFP}$, uninfected: $24.9 \pm 5.0 \mathrm{pA}$, infected: $32.4 \pm 5.7 \mathrm{pA}, \mathrm{p}=0.16$; PSD-95::GFP, uninfected: $75.4 \pm 9.3 \mathrm{pA}$, infected: $102.2 \pm 14.9 \mathrm{pA}, \mathrm{p}=0.06$ ). This result confirmed that the PSD-95 $\Delta \mathrm{GK}:: \mathrm{GFP}$ construct is functional and it can achieve the overexpression effect in the same way as full-length PSD-95 overexpression.

At the same time, we generated another construct to test the importance of GK domain by merging the GK domain to the first two PDZ domains of PSD-95 (sh95 + PDZ1/2-GK::GFP). This approach aimed at analyzing the effect of GK domain addition instead of its loss. Replacement of the endogenous PSD-95 with PDZ1/2-GK::GFP construct showed no significant change in AMPAR (uninfected: $-60.4 \pm 9.4 \mathrm{pA}$, infected: $-83.5 \pm 14.9 \mathrm{pA}, \mathrm{p}=0.10$ ) and NMDAR transmission of infected cells in comparison to control cells (uninfected: $21.0 \pm 2.8 \mathrm{pA}$, infected: $=22.6 \pm 3.7 \mathrm{pA}, \mathrm{p}=$ 0.68). These results were consistent with the data acquired from the sh95 + PSD-95 $\triangle$ PDZ3::GFP expressing neurons, as both of the constructs lacked the PDZ3 domain. However, having a partial rescue in the AMPAR transmission still generated an inconclusive result regarding the significance of GK domain. 
AMPAR NMDAR

A

PSD-95 $\triangle \mathrm{GK}:: G F P$
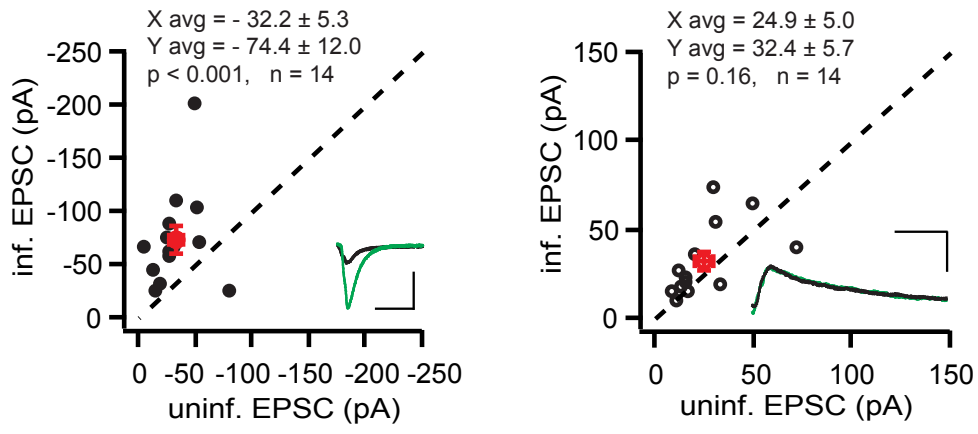

B
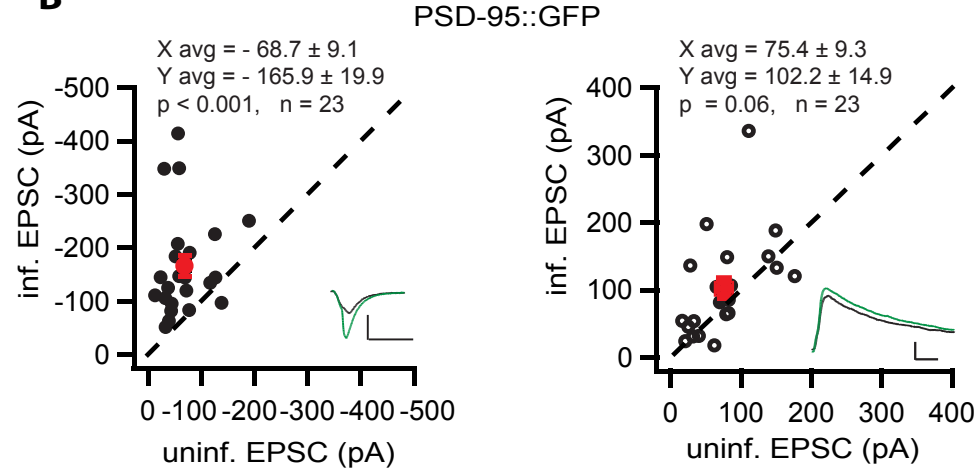

C

sh95 + PDZ1/2-GK::GFP
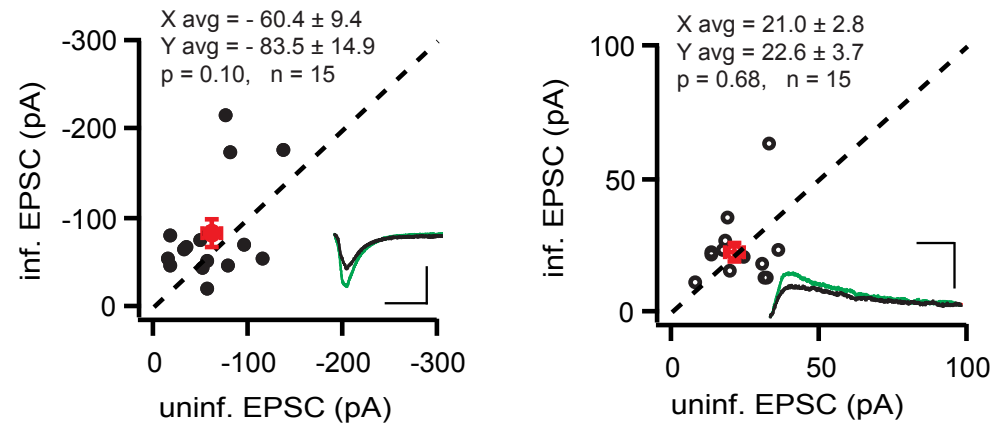

Figure 35: PSD-95 lacking the GK domain is functional and the addition of GK domain to PDZ1/2::GFP does not fully rescue the AMPAR transmission loss generated by PDZ1/2::GFP expression in CA1 region rat hippocampus.

(A) Overexpression of PSD-95 lacking GK domain significantly increases AMPAR responses of infected cells $(p<0.001)$, without affecting the NMDAR transmission $(p=0.16)$. (Performed by Stéphanie Bonnet)

(B) Overexpression of full-length PSD-95 significantly increases AMPAR responses of infected cells $(p<0.001)$, without changing the NMDAR transmission $(p=0.06)$. (Performed by Derya Akad)

(C) Replacement of endogenous PSD-95 by PDZ1/2-GK::GFP mutant does not alter AMPAR $(p=0.10)$ and NMDAR transmission $(p=0.68)$ of infected cells in comparison to uninfected cells. (Performed by Stéphanie Bonnet)

(The scale bars correspond to $50 \mathrm{pA}$ and $25 \mathrm{~ms}$. Green traces indicate the infected and the black traces indicate the control cell responses. The red marker corresponds to the average of all data points on the plot.) 
To yield a more conclusive result regarding the function of GK domain we tested the effect of sh95 + PSD-95 $\Delta \mathrm{GK}:$ :GFP construct in mouse hippocampal slice culture. We recorded first the pure expression of the shRNA construct against endogenous PSD-95 in hippocampal slice culture prepared from P8-9 mice. The recordings showed that the expression of sh95 significantly diminishes the AMPAR mediated responses by $20 \%$, without affecting the NMDAR mediated responses (AMPAR, uninfected: $-75.4 \pm$ 10.8 pA, infected: $-63.2 \pm 9.5 \mathrm{pA}, \mathrm{p}<0.05$; NMDAR, uninfected: $56.2 \pm 8.1 \mathrm{pA}$, infected: $47.3 \pm 7.5$ pA, p=0.08) (Figure 36A). In addition, expression of sh95 + PSD$95 \Delta \mathrm{GK}: \mathrm{GFP}$ construct resulted in a significant increase in AMPAR transmission. NMDAR transmission was not altered (AMPAR, uninfected: $-75.4 \pm 10.8 \mathrm{pA}$, infected: $-63.2 \pm 9.5 \mathrm{pA}, \mathrm{p}<0.05$; NMDAR, uninfected: $56.2 \pm 8.1 \mathrm{pA}$, infected: $47.3 \pm 7.5 \mathrm{pA}$, $\mathrm{p}=0.08)$ (Figure 36B). 
mouse
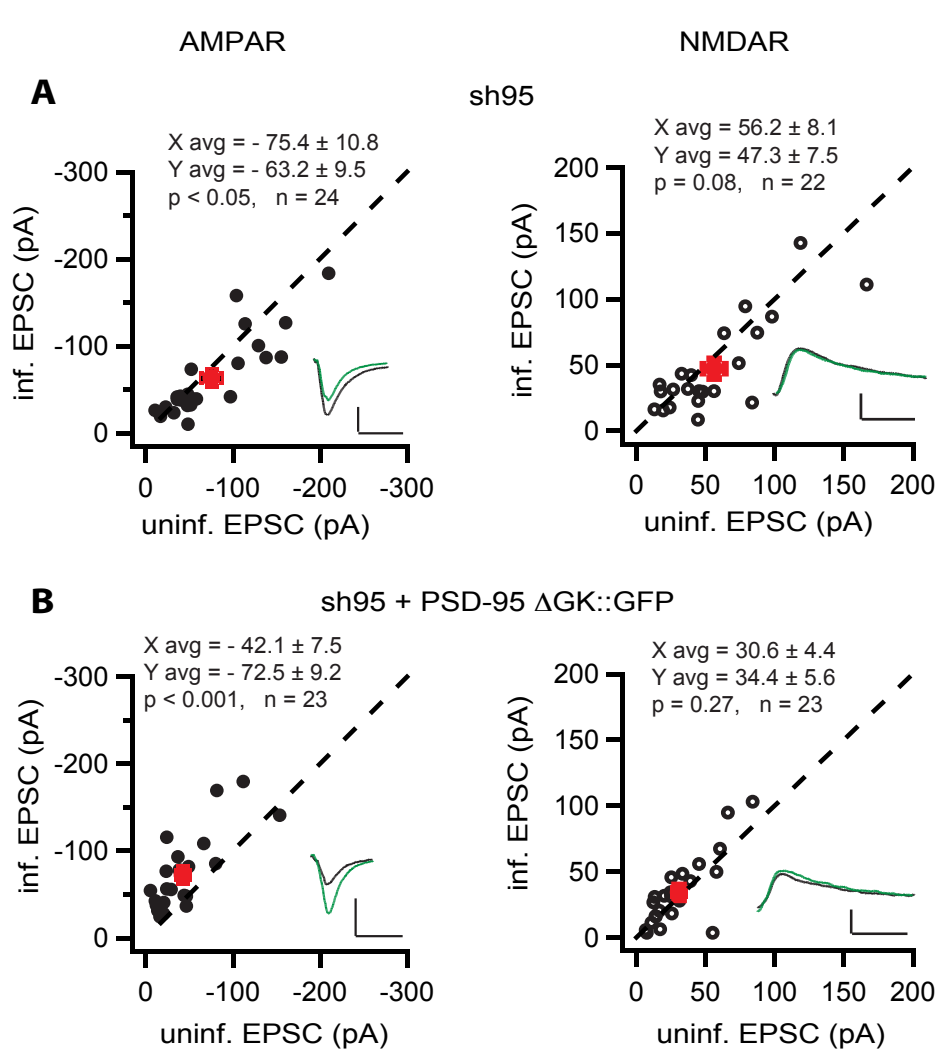

Figure 36: PSD-95 lacking the GK domain is functional in CA1 region neurons of mouse hippocampal slice culture.

(A) Expression of shRNA targeting endogenous PSD-95 decreased AMPAR responses by 20\% $(p<0.05)$ but does not alter the NMDAR responses $(p=0.08)$. (Performed by Derya Akad)

(B) Expression of sh95 + PSD-95 $\Delta \mathrm{GK}:$ :GFP construct enhanced the AMPAR mediated responses significantly $(p<0.001)$ and does not change the NMDAR transmission $(p=0.27)$. (Performed by Derya Akad)

(The scale bars correspond to $50 \mathrm{pA}$ and $25 \mathrm{~ms}$. Green traces indicate the infected and the black traces indicate the control cell responses. The red marker corresponds to the average of all data points on the plot.)

Since the results of sh95 + PSD-95 $\Delta \mathrm{GK}:: \mathrm{GFP}$, sh95 + PDZ1/2-GK::GFP and sh95 + PSD-95 $\triangle$ PDZ3::GFP expressions in rat slice culture showed neither significant increase nor significant decrease in AMPAR responses, whereas sh95 + PSD-95 $\Delta \mathrm{GK}:: \mathrm{GFP}$ expression in mouse culture showed a significant increase in AMPAR responses, we wanted to understand whether this phenotype is caused by the nonefficient knock-down of PSD-95 in rat hippocampal slice cultures. To eliminate this factor, we decided to analyze the effect of the mutants in PSD-95 knockout background. At first we tested the effect of PDZ1/2::GFP and the full-length PSD-95 (PSD95::GFP) construct on CA1 region neurons of PSD-95 knockout mouse hippocampal slice culture. 
As expected, the expression of PDZ1/2::GFP could not enhance the AMPAR (uninfected: - $70.6 \pm 12.0 \mathrm{pA}$, infected: $-66.6 \pm 14.4 \mathrm{pA}, \mathrm{p}=0.63$ ) and NMDAR responses of the infected cells in comparison to controls (uninfected: $110.6 \pm 20.9 \mathrm{pA}$, infected: $100.0 \pm 17.9 \mathrm{pA}, \mathrm{p}=0.25)$. PSD-95::GFP expression, on the other hand, increased the AMPAR transmission by 2.5 folds (uninfected: $-38.1 \pm 5.0 \mathrm{pA}$, infected: - $96.0 \pm 17.6 \mathrm{pA}, \mathrm{p}<0.05$ ) without changing the NMDAR transmission (uninfected: $83.3 \pm 22.7 \mathrm{pA}$, infected: $113.2 \pm 34.5 \mathrm{pA}, \mathrm{p}=0.065)$.

AMPAR NMDAR
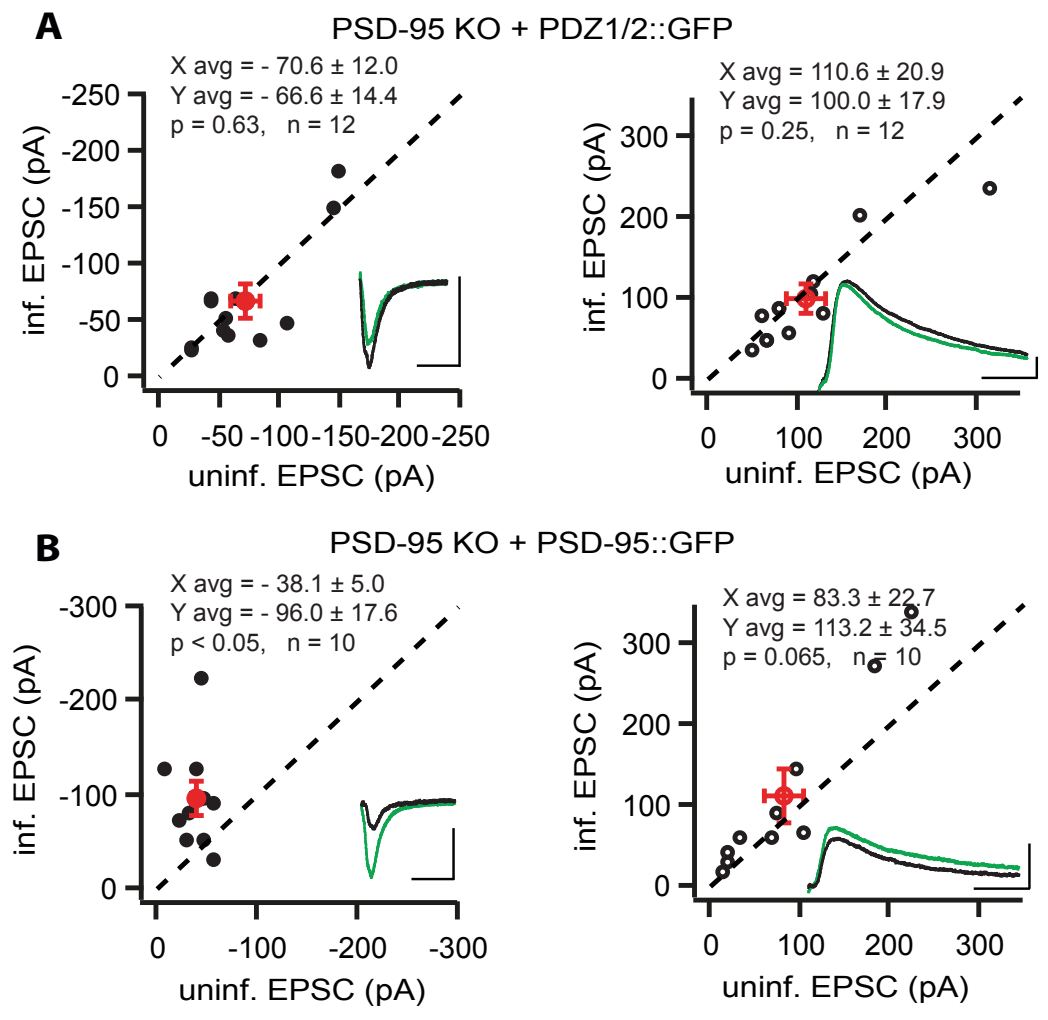

Figure 37: Full-length PSD-95 expression increases AMPAR responses in infected cells by 2.5 fold in CA1 region hippocampal PSD-95 knockout slices, whereas PDZ1/2::GFP expression does not enhance the AMPAR transmission.

(A) Expression of PDZ1/2::GFP construct in CA1 region neurons of PSD-95 knockout slices did not alter the AMPAR $(p=0.63)$ and NMDAR transmission $(p=0.25)$ of the infected cells. (Performed by Stéphanie Bonnet)

(B) Expression of full-length PSD-95 construct in CA1 region neurons of PSD-95 knockout slices significantly enhanced the AMPAR transmission $(\mathrm{p}<0.05)$ without a change in NMDAR transmission $(\mathrm{p}=0.065)$. (Performed by Stéphanie Bonnet)

(The scale bars correspond to $50 \mathrm{pA}$ and $25 \mathrm{~ms}$. Green traces indicate the infected and the black traces indicate the control cell responses. The red marker corresponds to the average of all data points on the plot.) 
After confirming the control conditions by the expression of PDZ1/2::GFP and PSD-95::GFP constructs, we tested the effect of PSD-95 $\Delta$ PDZ3::GFP and PSD-95 $\Delta \mathrm{GK}:: \mathrm{GFP}$ mutants on the basal synaptic transmission in hippocampal PSD-95 knockout slice culture. Interestingly, expression of these constructs significantly increased the AMPAR transmission of the infected cells (PSD-95 $\Delta$ PDZ3::GFP, uninfected: $-78.4 \pm 12.3$ pA, infected: $-102.9 \pm 18.3$ pA, p<0.05; PSD-95 $\Delta$ GK::GFP, uninfected: $-51.9 \pm 7.8$ pA, infected: $-120.5 \pm 22.5 \mathrm{pA}, \mathrm{p}<0.05)$, without altering the NMDAR transmission (PSD-95 $\triangle$ PDZ3::GFP, uninfected: $60.1 \pm 12.0 \mathrm{pA}$, infected: $54.6 \pm 9.9$ pA, p=0.37; PSD-95 $\Delta \mathrm{GK}:: G F P$, uninfected: $94.5 \pm 11.8$ pA, infected: 121.7 $\pm 21.9 \mathrm{pA}, \mathrm{p}=0.20)$.
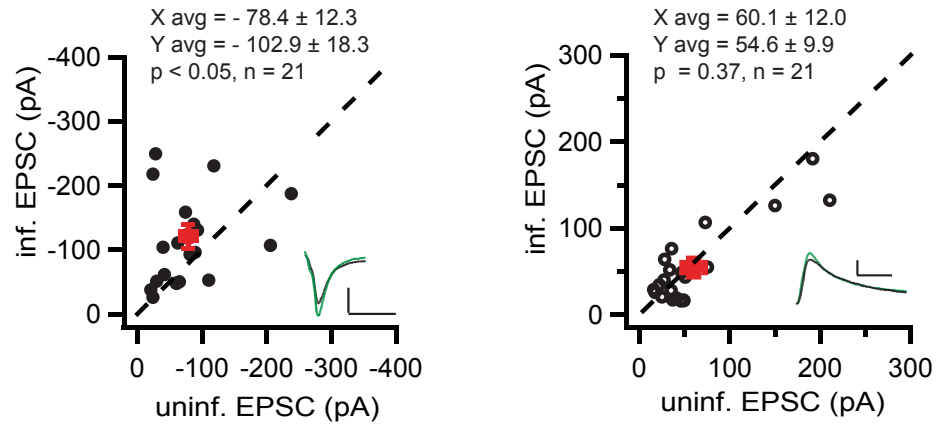

B

PSD-95 KO + PSD-95 $\triangle G K:: G F P$
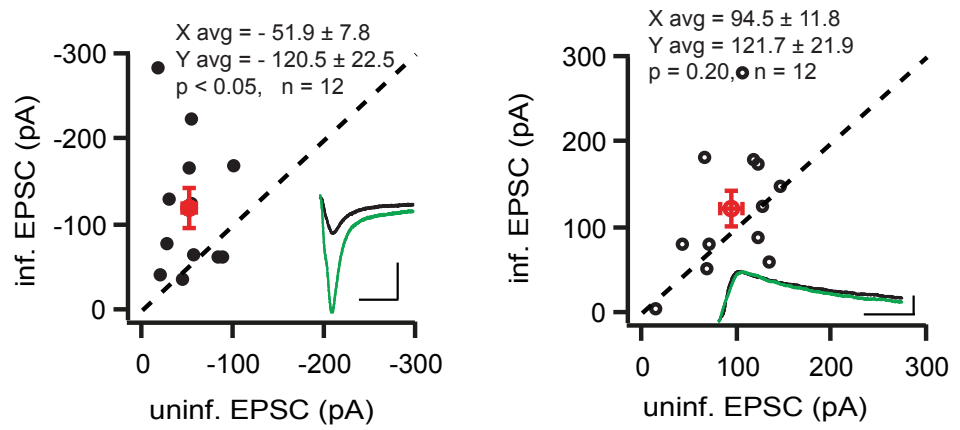

Figure 38: PSD-95 mutants lacking PDZ3 or GK domain enhance AMPAR mediated transmission in PSD-95 knockout background without altering the NMDAR transmission.

(A) Expression of sh95 + PSD-95 $\Delta$ PDZ3::GFP construct in PSD-95 knockout background significantly increased AMPAR mediated responses $(p<0.05)$ but does not change NMDAR mediated responses $(\mathrm{p}=0.37)$. (Performed by Derya Akad)

(B) Expression of PSD-95 $\Delta \mathrm{GK}:$ :GFP construct in PSD-95 knockout background significantly increased AMPAR mediated responses $(\mathrm{p}<0.05)$ but does not change NMDAR mediated responses $(\mathrm{p}=0.20)$. (Performed by Stéphanie Bonnet)

(The scale bars correspond to $50 \mathrm{pA}$ and $25 \mathrm{~ms}$. Green traces indicate the infected and the black traces indicate the control cell responses. The red marker corresponds to the average of all data points on the plot.) 
The difference between the results obtained from the rat and PSD-95 knockout mouse slices raised the question to whether there is a compensatory mechanism taking place between within MAGUK family. It was shown that the absence of one or more MAGUK members can be compensated by the elevated levels of other MAGUKs (Elias et al., 2006). In order to test this possibility, we checked the levels of different MAGUKs in the PSD fraction of PSD-95 knockout, PSD-93 knockout and wild-type animals. The results demonstrated that SAP102 and SAP97 but not PSD-93 levels were significantly increased in the PSD fraction of adult PSD-95 knockout mice (Figure 39). There was no difference in the levels of SAP102, SAP97 and PSD-95 in PSD-93 knockout mice (SAP97: $\mathrm{F}(2,11)=21.44, \mathrm{p}<0.001$, one-way ANOVA; wt, $100 \pm 8.0, \mathrm{n}$ $=4$ vs. p95 KO, $174.3 \pm 9.9, \mathrm{n}=6, \mathrm{p}<0.001$; Bonferroni post-test; wt vs. p93 KO, $114.4 \pm 5.3, \mathrm{n}=4, \mathrm{p}>0.05$; Bonferroni post-test; SAP102: F2,7 = 17.50, $\mathrm{p}<0.01$, oneway ANOVA; wt, $100 \pm 4.0, \mathrm{n}=4$ vs. p95 KO, $187.6 \pm 16.4, \mathrm{n}=3, \mathrm{p}<0.01$; Bonferroni post- test; wt vs. p93 KO, $115 \pm 13.2, \mathrm{n}=3, \mathrm{p}>0.05)$.

A

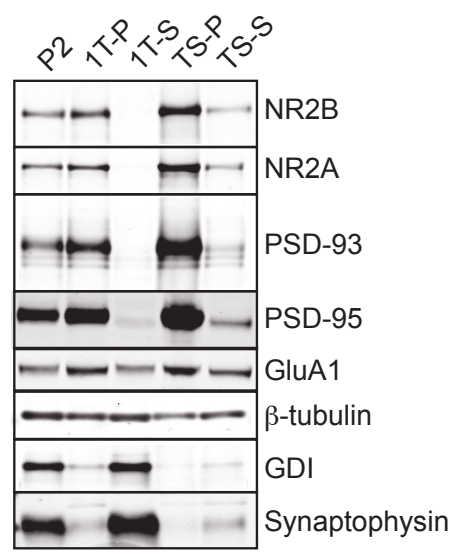

B
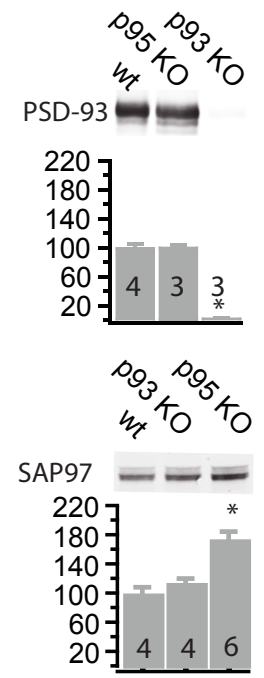
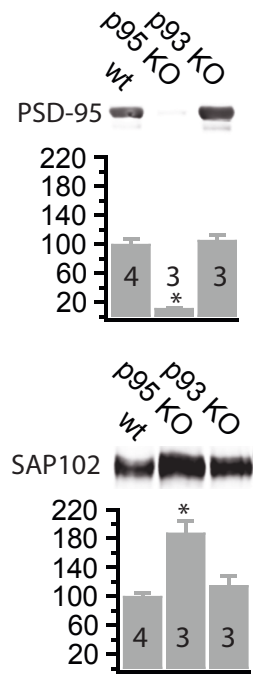

Figure 39: SAP102 and SAP97 levels are elevated in the PSD fraction of PSD-95 knockout animals. Cortices of PSD-95 knockout, PSD-93 knockout and wild-type mice were subjected to subcellular fractionation. The proteins in different fractions were immunoblotted with indicated antibodies. (Performed by Yanling Liu)

(A) Different fractions obtained after detergent treatment and the centrifugation steps are presented. The levels of synaptic proteins are indicated in the fractions.

(B) Quantification of MAGUK members in the PSD fraction of wild-type, PSD-95 knockout and PSD-93 knockout animals. For the statistical analysis one-way ANOVA with Bonferroni posttest were used. The graphs present the mean \pm SEM values. 
Encouraged by these results, we decided to test the involvement of SAP102 and SAP97 in the basal synaptic transmission of CA1 region PSD-95 knockout neurons. We combined the expression of PSD-95 domain mutants with the knock-down of endogenous SAP102 or endogenous SAP97. Knock-down of SAP102 in hippocampal slices prepared from PSD-95 knockout animals did not alter the AMPAR and NMDAR transmission of infected cells (AMPAR, uninfected: $-96.5 \pm 22.1 \mathrm{pA}$, infected: $-79.6 \pm$ $14.7 \mathrm{pA}, \mathrm{p}=0.24$; NMDAR, uninfected: $69.1 \pm 11.8 \mathrm{pA}$, infected: $62.1 \pm 9.6 \mathrm{pA}$, $\mathrm{p}=0.11$ ). The expression of PSD-95 $\Delta \mathrm{GK}:$ :GFP mutant in combination with shRNA against SAP102 interestingly eliminated the significant increase in AMPAR transmission observed with PSD-95 $\Delta \mathrm{GK}::$ GFP expression alone (uninfected: - $46.6 \pm$ $6.7 \mathrm{pA}$, infected: $-56.8 \pm 7.1 \mathrm{pA}, \mathrm{p}=0.22$ ). There was a slight and significant increase observed with the NMDAR responses of sh102 + PSD-95 $\Delta \mathrm{GK}::$ GFP expressing cells (uninfected: $41.6 \pm 5.8 \mathrm{pA}$, infected: $57.7 \pm 9.1 \mathrm{pA}, \mathrm{p}<0.05$ ). In contrast to $\operatorname{sh} 102+$ PSD-95 $\Delta$ GK::GFP construct expression, combination of PSD-95 $\Delta \mathrm{GK}:$ :GFP expression with a shRNA against endogenous SAP97 did not eliminate the significant increase observed with PSD-95 $\Delta \mathrm{GK}::$ GFP expression alone (uninfected: - $87.7 \pm 10.5$ $\mathrm{pA}$, infected: $-123.9 \pm 13.5 \mathrm{pA}, \mathrm{p}=0.002$ ). There was no difference in NMDAR transmission of infected cells in comparison to control cells (uninfected: $68.4 \pm 8.3 \mathrm{pA}$, infected: $63.6 \pm 6.6 \mathrm{pA}, \mathrm{p}=0.65$ ). These results imply that the AMPAR response enhancement of PSD-95 $\Delta \mathrm{GK}::$ GFP expressing cells in PSD-95 knockout background is SAP102 but not SAP97 dependent. 

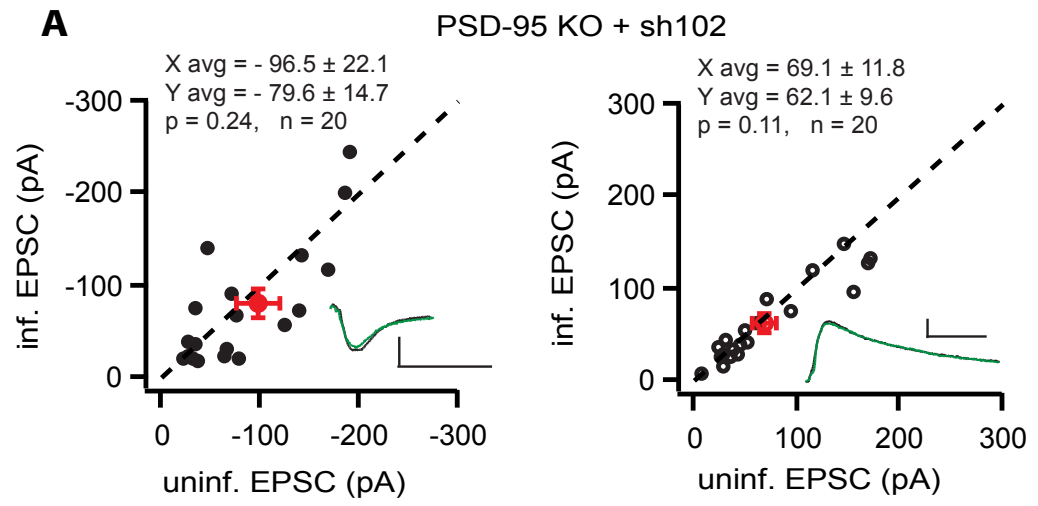

B

PSD-95 KO + sh102 + PSD-95 $\Delta$ GK::GFP
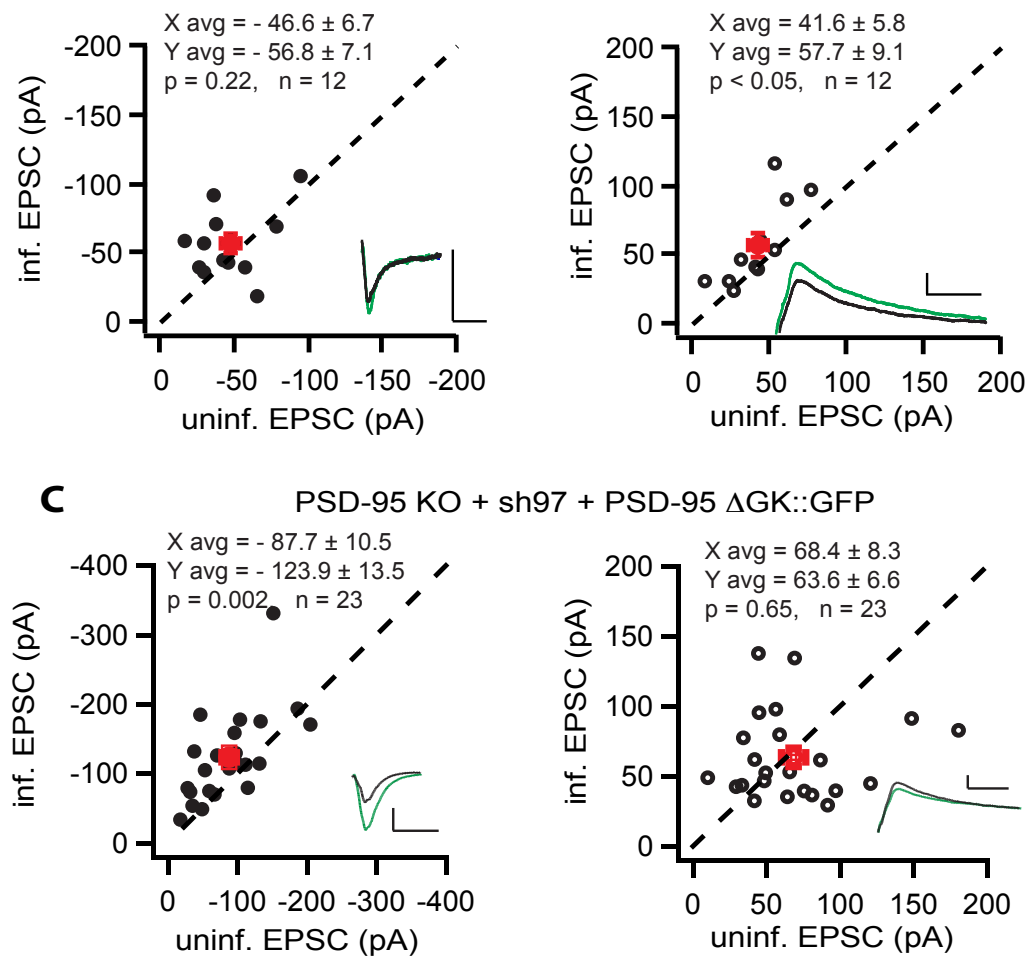

Figure 40: The AMPAR response enhancement of cells expressing GK domain lacking PSD-95 in PSD-95 knockout background is SAP102 dependent.

(A) Knock-down of endogenous SAP102 in PSD-95 knockout neurons did not alter the AMPAR $(\mathrm{p}=0.24)$ and NMDAR transmission $(\mathrm{p}=0.11)$. (Performed by Derya Akad)

(B) Expression of GK domain-lacking PSD-95 in combination with sh102 did not enhance AMPAR responses $(\mathrm{p}=0.22)$ but slightly increased NMDAR mediated responses $(\mathrm{p}<0.05)$. (Performed by Stéphanie Bonnet)

(C) Expression of GK domain-lacking PSD-95 in combination with sh97 significantly enhanced AMPAR responses $(p=0.002)$ but did not change NMDAR responses $(p=0.65)$. (Performed by Derya Akad)

(The scale bars correspond to $50 \mathrm{pA}$ and $25 \mathrm{~ms}$. Green traces indicate the infected and the black traces indicate the control cell responses. The red marker corresponds to the average of all data points on the plot.) 
We next tested the involvement of SAP102 in basal synaptic transmission mediated by PSD-95 $\Delta$ PDZ3::GFP and PDZ1/2-GK::GFP mutants in PSD-95 knockout CA1 region hippocampal neurons. Expression of PSD-95 $\triangle$ PDZ3::GFP in combination with the shRNA against SAP102 (sh102) did not enhance the AMPAR responses of infected cells (uninfected: $65.4 \pm 6.7 \mathrm{pA}$, infected: $77.7 \pm 10.8 \mathrm{pA}, \mathrm{p}=0.29$ ). NMDAR responses were slightly decreased in the infected cells (uninfected: $53.4 \pm 12.9 \mathrm{pA}$, infected: $40.3 \pm 4.3 \mathrm{pA}, \mathrm{p}=0.005$ ) (Figure 41A). The effect of sh102 on AMPAR and NMDAR responses suggested that the function of PSD-95 $\triangle$ PDZ3::GFP is also SAP102 dependent.

In addition, we expressed PDZ1/2-GK::GFP mutant in the presence and absence of SAP102 in PSD-95 knockout neurons. Similar to the other mutants, expression of PDZ1/2-GK::GFP alone increased the AMPAR transmission significantly while there was no change in NMDAR transmission of the infected cells (AMPAR, uninfected: $48.2 \pm 5.4$ pA, infected: $-92.0 \pm 13.9$ pA, $<<0.05$; NMDAR, uninfected: $61.2 \pm 11.3 \mathrm{pA}$, infected: $79.8 \pm 12.5 \mathrm{pA}, \mathrm{p}=0.86$ ) (Figure 41B). However, combining the mutant expression with sh102 did not abolish the increase in the AMPAR transmission, pointing out that the function of PDZ1/2-GK::GFP is not SAP102 dependent (AMPAR, uninfected: $-37.7 \pm 5.7 \mathrm{pA}$, infected: $-103.7 \pm 23.3 \mathrm{pA}, \mathrm{p}=0.003$; NMDAR, uninfected: $44.9 \pm 9.7 \mathrm{pA}$, infected: $55.6 \pm 17.1 \mathrm{pA}, \mathrm{p}=0.24)$ (Figure $41 \mathrm{C})$. 
PSD-95 KO + sh102 + PSD-95 $\triangle$ PDZ3::GFP
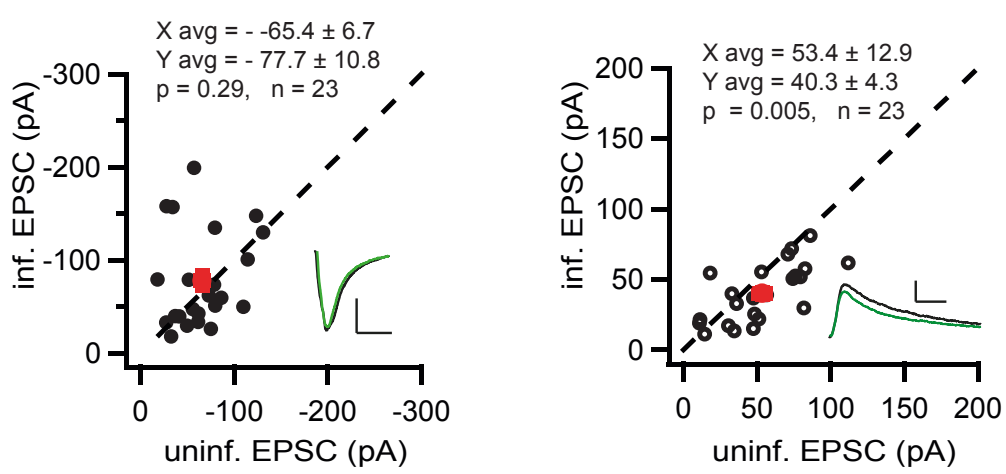

B

PSD-95 KO + PDZ1/2-GK::GFP
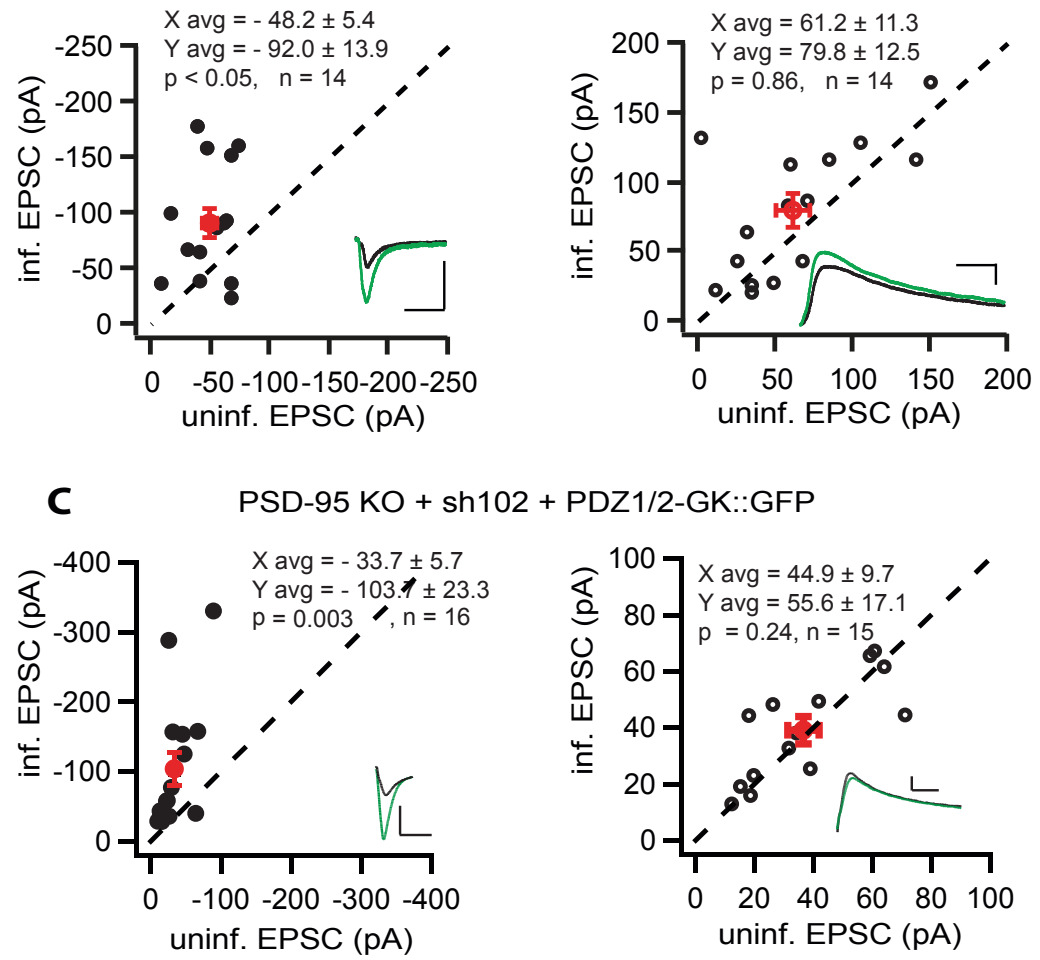

Figure 41: The function of PSD-95 lacking the PDZ3 domain, but not the function of PDZ1/2-

GK::GFP protein is SAP102 dependent in the CA1 region of PSD-95 knockout mouse hippocampus.

(A) Expression of PSD-95 $\triangle \mathrm{PDZ3}:$ GFP mutant in combination with shRNA against SAP102 (sh102) did not enhance AMPAR transmission $(\mathrm{p}=0.29)$, but caused a decrease in NMDAR transmission $(\mathrm{p}=0.005)$. (Performed by Derya Akad)

(B) Expression of PDZ1/2-GK::GFP mutant alone enhanced the AMPAR transmission of infected cells $(p<0.05)$, without changing the NMDAR transmission $(p=0.86)$. (Performed by Stéphanie Bonnet)

(C) Expression of the PDZ1/2-GK::GFP mutant in combination with sh102 maintains the increase in AMPAR transmission $(p=0.003)$ and does not affect the NMDAR transmission $(p=0.24)$. (Performed by Stéphanie Bonnet and Derya Akad)

(The scale bars correspond to $50 \mathrm{pA}$ and $25 \mathrm{~ms}$. Green traces indicate the infected and the black traces indicate the control cell responses. The red marker corresponds to the average of all data points on the plot.) 
Since both PSD-95 $\Delta$ PDZ3::GFP and PSD-95 $\Delta \mathrm{GK}:: \mathrm{GFP}$ constructs showed a significant increase in AMPAR transmission in neurons from the CA1 region of PSD95 knockout mice, we assumed that a PSD-95 form consisting of the first two PDZ domains and the SH3 domain could be sufficient to mediate basal synaptic transmission in PSD-95 knockout background. In order to this assumption, we generated a mutant of PSD-95, PDZ1/2-SH3::GFP, and expressed it in the CA1 region neurons of PSD-95 knockout hippocampal slices. The recordings showed a significant increase in both AMPAR and NMDAR transmission of the cells expressing PDZ1/2-SH3::GFP (AMPAR, uninfected: $-62.2 \pm 7.5 \mathrm{pA}$, infected: $-120.5 \pm 17.3 \mathrm{pA}, \mathrm{p}=0.004$; NMDAR, uninfected: $86.9 \pm 20 \mathrm{pA}$, infected: $112.6 \pm 22.2 \mathrm{pA}, \mathrm{p}=0.006$ ) (Figure 42A). The increase observed with the AMPAR and NMDAR transmission was dependent on SAP102, as combining the expression of PDZ1/2-SH3::GFP with shRNA against SAP102 in PSD-95 knockout neurons abolished the significant increases in AMPAR and NMDAR transmission (AMPAR, uninfected: $-70.5 \pm 10.6 \mathrm{pA}$, infected: $-93.6 \pm 18$ pA, p=0.17; NMDAR, uninfected: $57.3 \pm 12.9$ pA, infected: $61.4 \pm 9.4$ pA, p=0.67) (Figure 42B).

The results hitherto present two forms of minimal PSD-95, PDZ1/2-GK::GFP and PDZ1/2-SH3::GFP, which function similarly to the full-length PSD-95 in mediating AMPAR transmission in PSD-95 knockout neurons where SAP102 levels are upregulated. However, only PDZ1/2-SH3::GFP seems to be dependent on elevated SAP102 levels to be functional, whereas PDZ1/2-GK::GFP is not. 

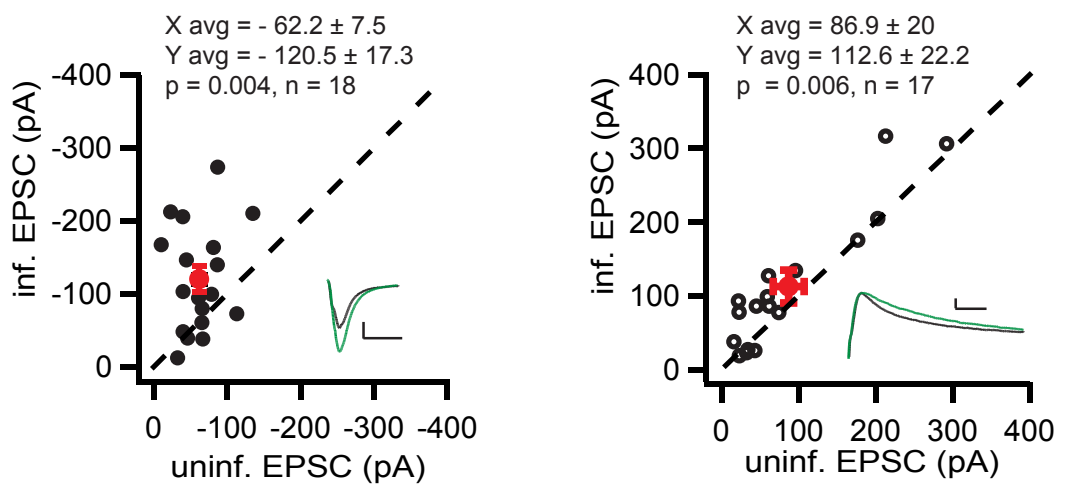

B
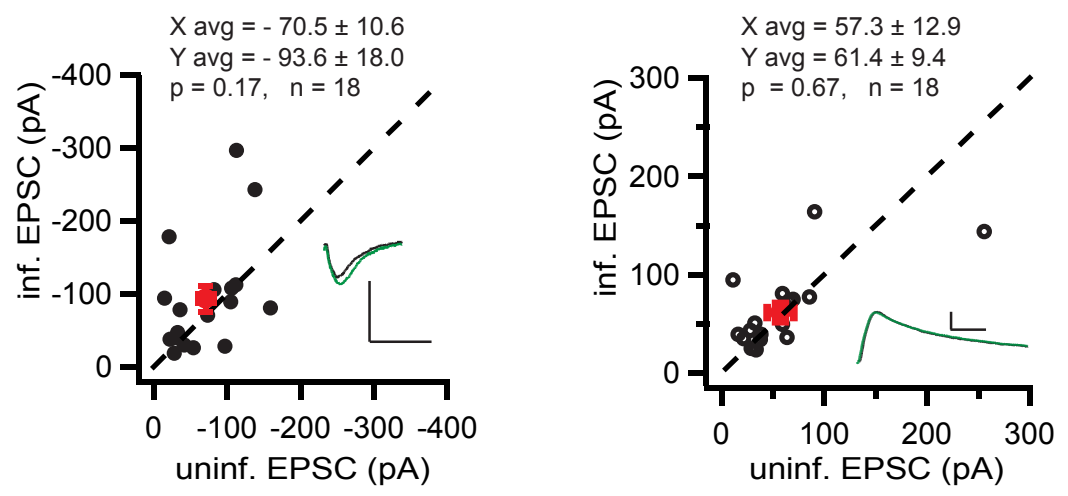

Figure 42: A minimal PSD-95 with the first two PDZ domains and the SH3 domain enhances basal synaptic transmission in CA1 region PSD-95 knockout neurons in a SAP102 dependent manner.

(A) Expression of PSD-95 with the first two PDZ domains and the SH3 domain significantly enhances the AMPAR ( $p=0.004)$ and NMDAR transmission of the infected neurons $(p=0.006)$. (Performed by Derya Akad)

(B) Expression of PSD-95 with the first two PDZ domains and the SH3 domain in combination with shRNA against SAP102 blocks the significant increases in AMPAR $(\mathrm{p}=0.17)$ and NMDAR responses $(\mathrm{p}=0.67)$ observed with PDZ1/2-SH3::GFP expression alone. (Performed by Derya Akad)

(The scale bars correspond to $50 \mathrm{pA}$ and $25 \mathrm{~ms}$. Green traces indicate the infected and the black traces indicate the control cell responses. The red marker corresponds to the average of all data points on the plot.) 



\section{Discussion}

\subsection{Important optimization steps in the generation of molecular replacement constructs}

Protein overexpression and molecular replacement techniques via lentiviral vectors are powerful tools to study the function of a protein on a single cell level (Schlüter et al., 2006). Commonly used vectors enable enhanced construct expression through a WPRE transcription enhancer cassette and allow the visualization of the proteins through the GFP-tag (Lois et al., 2002). Although these are helpful tools, they can generate drawbacks depending on the aim of the study.

During my project, expression of CDK5 phosphorylation site mutants of PSD-95 under the influence of the WPRE cassette exhibited enhancement of excitatory synaptic transmission for both of the mutants, although they mimicked opposite phosphorylation states (Figure 12). Discarding the WPRE cassette brought the expression of the constructs to the endogenous levels and unmasked the specific effects of the mutants on AMPAR and NMDAR transmission in rat CA1 region hippocampal neurons (Figure 13, Figure 16). In addition, the expression of the mutants with a GFP-tag interfered strongly with the PSD-95 - Src kinase interaction (Figure 14). This interference could be avoided by expressing PSD-95 mutants and GFP separately in a bicistronic construct. GFP is recommended as a reporter to detect the localization of the proteins and help to quantify the expression levels due to its small size and non-toxicity. However, it might still induce conformational changes of the constructs and alter the protein processing and flexibility (Cabantous et al., 2005). Thus, in my study I could demonstrate the importance of the expression level and the usage of the GFP-tag as two important factors to consider when using lentiviral replacement vectors. 


\subsection{CDK5 phosphorylation site mutants of PSD-95 have different effects on AMPAR and NMDAR transmission}

CDK5 is a proline-directed serine/threonine kinase which is implicated with its effect in the development of the nervous system, migration of the cortical neurons and lamination of the cortex (Chae et al., 1997; Fang et al., 2011). Besides its function in the development, CDK5 is also known to be an important regulator of the synaptic transmission, such that its malfunction is indicated in neurodegenerative diseases as in Alzheimer's disease (Cruz and Tsai, 2004; Malenka and Malinow, 2011). The importance of CDK5 arises from its ability to phosphorylate various pre- and postsynaptic proteins, one of which is PSD-95 (Morabito et al., 2004; Cheung et al., 2006). CDK5 and its co-activator p35 are highly enriched in the postsynaptic membranes (Humbert et al., 2000; Niethammer et al., 2000). PSD-95 is phosphorylated on three N-terminal aminoacid residues by CDK5 in hippocampal neurons, T19, S25 and S35 (Morabito et al., 2004). The localization of these phosphorylation sites is critical, since two of the serine residues are part of the putative PEST sequence in the N-terminus of PSD-95. PEST sequence was indicated to be responsible of PSD-95 ubiquitination (Colledge et al., 2003), which recently has been correlated with nonproteosomal pathways and with no change in PSD-95 protein levels (Bianchetta et al., 2011). However, in the absence of the PEST sequence, PSD-95 could not form dimers and enhance AMPAR responses in rat hippocampus (Xu et al., 2008). Thus, the CDK5 phosphorylation state of PSD-95 might be an important determinant of its function. In consistency with this idea, the absence of CDK5 phosphorylation on PSD-95 or the blockade of the endogenous CDK5 activity demonstrated an increase in PSD-95 cluster size in cultured neurons (Morabito et al., 2004). However the functional consequence of such a phosphorylation mechanism was not examined.

Here, I could demonstrate that the CDK5 phosphorylation state of PSD-95 indeed has a functional consequence on the AMPAR and NMDAR transmission in the neurons of the rat hippocampus CA1 region. While the replacement of the endogenous PSD-95 by the non-phosphorylatable mutant had no effect on AMPAR and NMDAR transmission, phospho-mimicking mutant replacement significantly enhanced both AMPAR and NMDAR transmission by $30-40 \%$ (Figure 16). The increase in both 
components might indicate a role of the phospho-mimicking mutant in the induction or stabilization of new synapses. Interestingly, as it will be discussed in section 4.5, blocking neuronal activity for 2 days by TTX caused a 4-fold increase in the AMPAR transmission of the phospho-mimicking mutant expressing neurons, pointing to a potential effect on synapse unsilencing (Figure 24). This effect could be tested by measuring the miniature EPSC (mEPSC) frequency of the phospho-mimicking mutant expressing neurons and analyzing the AMPA/NMDA EPSC ratio via minimal stimulation.

These results were different from the imaging data presented by Morabito et al. 2004, which showed that the non-phosphorylatable mutant of PSD-95 formed larger clusters at the synapse. Even though clustering does not necessarily need to reflect the effects on the function, the difference in the results may arise from the experimental conditions used in the studies. Imaging experiments by Morabito et al., 2004 were performed on dissociated hippocampal neuron cultures and the immunostaining results were obtained after 18-20 hours of viral infection (Morabito et al., 2004). On the other hand, electrophysiological recordings I performed were performed in hippocampal slice culture system after 5 days of the viral infection. The difference in the timing can reveal a temporal effect of CDK5 phosphorylation on PSD-95. Another difference could be due to a pathway specific effect of CDK5 phosphorylation on PSD-95. AMPAR and NMDAR transmission of CA1 region neurons were measured by specifically stimulating the Schaffer collaterals, whereas Morabito et al., 2004 demonstrated a global effect of the CDK5 phosphorylation on PSD-95 regarding the cluster size. The possibility of having a pathway specific effect could be addressed by performing miniature EPSC recordings on CA1 region neurons. This method reveals the collective input from all synapses a neuron receives instead of the contribution from a single pathway. mEPSC recordings did not show a difference between the control and nonphosphorylatable mutant expressing cells regarding the mEPSC amplitude and frequency (Figure 26). Interestingly, miniature EPSC recordings from the phosphomimicking mutant expressing neurons exhibited a significant decrease in the amplitude of mEPSC events, while the frequency was not altered (Figure 26). In both cases the control cell amplitude and frequencies were equal, suggesting that the difference 
observed in the amplitude is certainly mutant specific. The mEPSC results from the phospho-mimicking mutant expressing neurons were different from the evoked EPSC data, implying that there might be a pathway specific effect of CDK5 phosphorylation on PSD-95. Nevertheless, there are additional factors which might cause this difference. First, it was shown that the synapse and AMPA receptor number change according to the distance from the soma (Andrasfalvy and Magee, 2001; Nicholson et al., 2006). This might be an important factor to consider while comparing evoked and mEPSC events. Second, recording evoked responses targets and stimulates the same synaptic pathway, which may cause the stabilization of these particular synapses (Groc et al., 2006a). Lastly, Sara et al., 2011 demonstrated the distinction between two pools of AMPA receptors mediating evoked and spontaneous glutamatergic transmission, which will be targeted separately during the mEPSC recordings.

In addition, unaltered $\mathrm{mEPSC}$ frequency of phospho-mimicking mutant expressing neurons undermines the assumption that this mutant has an impact on synapse unsilencing. However, one should take into consideration that a change of $30 \%$ in evoked AMPAR and NMDAR responses might not be detected in mEPSC recordings. To get a better understanding about the abundance of the silent synapses, one can apply minimal stimulation protocol and analyze the AMPA/NMDA EPSC ratio.

Another possibility that could explain the difference between the imaging and the electrophysiological results would be non-phosphorylatable mutant of PSD-95 affecting the extrasynaptic receptor pool hence forming bigger clusters without affecting the AMPAR transmission. In order to test this possibility, I performed glutamate spill-over experiments on the non-phosphorylatable mutant expressing cells. The results did not show a difference between the infected and control cells (Figure 28), making the hypothesis of non-phosphorylatable mutant of PSD-95 affecting the extrasynaptic AMPA receptor pool very unlikely.

Lastly, as I could demonstrate, high protein expression levels can mask the effect of the mutant constructs. In my experiments, replacement of endogenous PSD-95 by the non-phosphorylatable mutant under the influence of WPRE cassette resulted in a 2fold increase in AMPAR responses, similar to the PSD-95 overexpression effect (Figure 12). However, the replacement construct with the endogenous levels of 
expression abolished this increase (Figure 16). In the study of Morabito et al., 2004 non-phosphorylatable mutant of PSD-95 was overexpressed in dissociated neuron culture. Considering the fact that a similar difference in the synaptic transmission could be reflected on the cluster size of PSD-95, replication of the imaging experiments with replacement constructs exhibiting endogenous levels of expression will be important.

\subsection{Endogenous CDK5 activity manipulation to confirm the mutation effects}

It is important to confirm that the mutations generated on the proteins indeed reflect the in vivo changes of the phosphorylation state. To address this point, I overexpressed the dominant-negative form of CDK5 (DNK5) and CDK5 co-activators p25 and p35 in CA1 region neurons, in order to compare them to the non-phosphorylatable and phospho-mimicking mutants of PSD-95 respectively. Expression of DNK5 showed no change in AMPAR and NMDAR transmission in consistency with the data obtained from the non-phosphorylatable mutant (Figure 17). In contrast, the enhancement in AMPAR and NMDAR transmission observed with the phospho-mimicking mutant was absent when p35 and p25 were expressed (Figure 17). Even though co-activator expression did not support the phenomenon observed with the phospho-mimicking mutant, we do not know whether the co-activators could accomplish CDK5 overactivation. This point can be tested by blotting the targets of the CDK5, such as Nudel protein, with and without co-activator overexpression (Niethammer et al., 2000). In addition, several studies enhance the endogenous CDK5 activity via the expression of a p35/CDK5 co-expression construct instead of the co-activator expression alone (Tsai et al., 1994; Van den Haute et al., 2001; Morabito et al., 2004; Zhang et al., 2008). Thus, it might be necessary to generate a construct as the p35/CDK5 co-expression. To prove that the mutations generated on PSD-95 indeed reflect the phosphorylation regulation by $\mathrm{CDK} 5$, expression of the phospho-mimicking mutant together with DNK5 and the non-phosphorylatable mutant together with p35/CDK5 construct will be crucial.

In such a case, it will be crucial to differentiate the effect of CDK5 phosphorylation mutants of PSD-95 from the effect of CDK5 activity itself, because CDK5 was shown 
to phosphorylate GluN2A subunit of NMDA receptors at S1232 residue (Li et al., 2001). In order to differentiate these effects, GluN2A subunit phosphorylation can be quantified via immunoblotting when the phosphorylation mutants of PSD-95 are expressed in dissociated neuronal cultures in the presence and absence of CDK5 overactivation.

\subsection{Src kinase family is involved in the regulation of} AMPAR and NMDAR transmission mediated by CDK5 phosphorylation site mutants of PSD-95

Recently, a mechanism regulating the tyrosine phosphorylation of NMDA receptors was proposed by Zhang et al., 2008. The mechanism involved the binding of Src kinase to PSD-95 in a CDK5 phoshorylation state-dependent manner. This binding was followed by the phosphorylation of the Y1472 residue on GluN2B subunit phosphorylation, enhancing the surface expression of GluN2B-containing NMDA receptors in hippocampal neurons (Zhang et al., 2008). In addition, it is well known that Src kinase family members not only phosphorylate NMDA receptor subunits but also AMPA receptor subunits (Hayashi and Huganir, 2004; Kalia et al., 2004). Although Zhang et al., 2008 displayed the GluN2B phosphorylation by Src kinase in a CDK5 phosphorylation state-dependent fashion, the effect of the interaction between Src kinase and the CDK5 phosphorylation mutants of PSD-95 on AMPA and NMDA receptor function was not shown. With my study, I could demonstrate that the effect of the phospho-mimicking mutant of PSD-95 on AMPAR and NMDAR transmission is Src kinase family-dependent (Figure 18). This result also reveals a new mechanism to control AMPAR transmission through the interaction of PSD-95 and Src kinase, which is CDK5 phosphorylation state-dependent. Moreover, I could show that the enhancement observed in NMDAR transmission with the phospho-mimicking mutant is GluN2B subunit specific (Figure 19). However, electrophysiological recordings cannot reveal whether this enhancement is due to an increase in the phosphorylation levels of GluN2B or in the total numbers of GluN2B-containing receptors. Immunoblotting of the total GluN2B and phospho-Y1472 from the phospho-mimicking mutant expressing 
cells demonstrated a trend towards an increase in GluN2B phosphorylation without a change in the total numbers of GluN2B (Figure 20). Even though the difference in the phosphorylation level is not significant yet, it points to an effect of phospho-mimicking mutant on NMDAR transmission which might go through the regulation of GluN2B phosphorylation. To confirm this hypothesis, $\mathrm{N}$ numbers for each group should be increased.

In order to specify which Src kinase family member is responsible for the enhancement in AMPAR and NMDAR transmission mediated by the phosphomimicking mutant of PSD-95, I activated PAC1R and D1 receptor pathways, which were shown to activate Src and Fyn kinases respectively (Yang et al., 2012). Stimulation of PAC1Rs brought the NMDAR transmission of control cells to the same levels as phospho-mimicking mutant expressing cells (Figure 21). This suggests that the enhancement in NMDAR transmission with the phospho-mimicking mutant expressing cells might be Src kinase-dependent. Stimulation of D1 receptors, on the other hand, brought both AMPAR and NMDAR transmission of the control cells to the same level as the phospho-mimicking mutant expressing cells (Figure 22). These results imply that there might be an overlap between the pathways of the phosphomimicking mutant effect and D1 receptor activation involving Fyn kinase activity. At the same time, stimulation of PAC1Rs affected the non-phosphorylatable mutant expressing and control cells in the same rate, as there was no significant difference between the infected and control cell AMPAR and NMDAR transmission (Figure 21). However, activation of D1 receptors caused an enhancement in both AMPAR and NMDAR transmission of non-phosphorylatable mutant expressing cells (Figure 22). This phenotype might imply that the non-phosphorylatable mutant of PSD-95 is more susceptible to the D1 receptor thus Fyn kinase activation.

However, it is not trivial to dissect the role of single Src kinase members in the regulation of AMPAR and NMDAR transmission mediated by the mutants, via stimulation of PAC1R and D1 receptor pathways, because stimulation of PAC1Rs and D1 receptors were shown to activate several other proteins besides Fyn and Src kinase. Activation of PAC1Rs in CA1 region neurons were shown to increase AMPAR mediated transmission through pathways involving cAMP and PKA activation (Costa 
et al., 2009). PAC1R activation also enhances NMDAR receptor function through the secondary messenger pathways activating PKC and Src kinase (Macdonald et al., 2005) or through the exposure of GluN2B Y1474 residue for the phosphorylation by Fyn kinase due to the dissociation of Rack1 and NMDAR complex (Yaka et al., 2003). At the same time, application of PAC1R agonist, PACAP38, was shown to modulate the NMDA receptor function independent of the secondary messenger pathways (Liu and Madsen, 1997). Similarly, stimulation of D1 receptors modify NMDAR transmission in PKA and PKC dependent pathways (Lee et al., 2002a). Taking into account that both AMPA and NMDA receptor subunits possess PKA and PKC phosphorylation sites, it is hard to conclude the direct effect of Src and Fyn kinases on the basal synaptic transmission mediated by CDK5 phoshorylation mutans. A better approach would be the use of shRNA constructs against endogenous Src and Fyn kinases or the use of pYEEI peptide activating Src kinases (Yu et al., 1997) to expose their direct role on the function of AMPA and NMDA receptors. Combination of the shRNA constructs with the CDK5 phosphorylation mutants of PSD-95 would reveal the specific Src kinases the mutants interact with.

\subsection{CDK5 phosphorylation site mutants of PSD-95 respond to activity changes differently}

NMDA receptor subunits, GluN2A and GluN2B, have been indicated with their role in changing the threshold of long-term potentiation and depression, and the ratio of GluN2A $\backslash$ GluN2B has been shown to modulate the plasticity of neurons (Liu et al., 2004a; Fox et al., 2006; Cho et al., 2009). Since phospho-mimicking mutant of PSD-95 exhibited significant increases both in AMPAR and NMDAR transmission (Figure 16), we asked whether these synapses start from a 'potentiated' state which gives rise to the enhanced AMPAR responses. However, blocking the GluN2B-containing NMDA receptors of phospho-mimicking mutant expressing neurons for two days did not abolish the enhancement in AMPAR transmission (Figure 23), indicating that the increase in AMPAR and NMDAR transmission is independent of each other.

It was shown by Zhang and Lisman, 2012 that the overexpression effect of PSD-95 is activity-dependent. We also hypothesized that the phospho-mimicking mutant of 
PSD-95 could contribute to the new synapse generation and stabilization. As PSD-95 was shown to be involved in synaptic maturation (Okabe et al., 1999; El-Husseini et al., 2000c; Losi et al., 2003b), we wanted to see whether the expression of the phosphorylation mutants in a state, where the synaptic activity and maturation are blocked, would have an impact.

Blockade of the neuronal activity for two days by TTX application enhanced the AMPAR transmission of phospho-mimicking mutant expressing cells dramatically (Figure 24). There was no difference observed between the non-phoshorylatable mutant expressing and control neurons regarding the AMPAR and NMDAR responses after the TTX treatment (Figure 24). It was shown by Turrigiano et al., 1998, that synaptic scaling is a mechanism to maintain the homeostasis of a neuronal system in response to the changes in the network activity. Chronic blockade of neuronal activity was shown to increase the mEPSC amplitudes, which was correlated with AMPA receptor function (Turrigiano, 2008). Follow up studies demonstrated that the PSD-95 abundance is an important factor for scaling down the synaptic activity. But for scaling up, the abundance and presence of PSD-95 was not crucial (Sun and Turrigiano, 2011). Here in my study, the dramatic increase in the AMPAR transmission of phospho-mimicking mutant expressing neurons after the activity blockade might suggest two options. First, PSD-95 might be involved in the homeostatic plasticity, particularly in scaling up, in a CDK5 phosphorylation state-dependent manner and this could be detected in an increase in mEPSC amplitudes after the activity blockade. Second, expression of the phospho-mimicking mutant of PSD-95 could induce silent synapse formation, upon activity blockade (Arendt et al., 2013). This effect can be demonstrated in an increase in mEPSC frequency after the activity blockade.

A second activity manipulation described by Zhang and Lisman, 2012 involved the blockade of mGluR5 receptors to block the overexpression effect of PSD-95. In addition, mGluR5 was proposed to regulate the NMDAR subunit switch in neurons of hippocampus and visual cortex (Matta et al., 2011). In order to test the effect of mGluR5 activity on the regulation of AMPAR and NMDAR transmission mediated by CDK5 phosphorylation mutants of PSD-95, I used MPEP, a selective mGluR5 antagonist. Application of MPEP for two days affected the basal synaptic transmission 
properties of the phosphorylation mutant expressing neurons differently. MPEP application abolished the increase in NMDAR transmission of phospho-mimicking mutant expressing neurons and enhanced the AMPAR transmission of nonphosporylatable mutant expressing neurons (Figure 25). It is known that mGluRs modulate the AMPAR and NMDAR function in different brain regions, including hippocampus (Rojas and Dingledine, 2013). mGluR5 was shown to modulate AMPA receptor endocytosis (Lüscher and Huber, 2010). Thus, it is possible that the nonphosphorylatable mutant of PSD-95 is resistant to the influence of mGluR5 activity, which prevents the AMPAR endocytosis upon mGluR5 activation. In addition, group 1 mGluRs were shown to enhance NMDAR transmission in hippocampus through Src kinase (Benquet et al., 2002). The result from the phospho-mimicking mutant expressing cells shows that the mGluR5 blockade eliminates the significant increase in the NMDAR transmission of the infected cells. This might suggest an overlapping pathway for mGluR5 and phospho-mimicking mutant action which involves the Src kinase activity. However, as mentioned before for PAC1R and D1 receptor activation, stimulation of mGluR5 triggers pathways not only involving Src kinase but also PKC activity (Benquet et al., 2002; Rojas and Dingledine, 2013). Therefore, it is not clear which kind of effect mGluR5 blockade would have on the basal synaptic transmission properties of the CDK5 phosphorylation mutants of PSD-95.

Nevertheless, the activity manipulation via TTX and mGluR5 blockade affects the mutants differently, rendering them as potential candidates not only for the regulation of the basal synaptic transmission, but also for the synaptic plasticity.

Besides its effect on basal synaptic transmission and synaptic plasticity, PSD-95 was shown to be involved in the pathogenesis of several neurodegenerative disorders, one of them being Alzheimer's disease. It was shown that PSD-95 levels are reduced in the pathology of the Alzheimer's disease (Roselli et al., 2005; Liu et al., 2010). Overactivation of NMDA receptors was indicated to be the cause of the synaptic loss of PSD-95 which was accompanied by a decrease in the GluA2 levels (Roselli et al., 2005; Chang et al., 2006; Liu et al., 2010). Interestingly, Roselli et al. 2005, demonstrated that in rat cortical neurons, amyloid-beta induced NMDAR activation causes PSD-95 loss in a CDK5 activity-dependent manner. Blocking the CDK5 activity 
or expressing the non-phosphorylatable mutant of PSD-95 could eliminate the PSD-95 loss upon amyloid-beta application (Roselli et al., 2005). In addition, it was shown that the application of amyloid-beta, a component of the amyloid plaques observed in the disease pathology, enhances the phosphorylation of GluN2A subunit of NMDA receptors and the association of GluN2A, PSD-95 and Src kinase complex (Wu and Hou, 2010). Stimulation of this complex is one of the triggers leading to neurodegeneration due to NMDAR overactivation.

Thus, besides their effect on the basal synaptic transmission, CDK5 phosphorylation mutants of PSD-95 may contribute to the pathogenesis of Alzheimer's disease through mediating the AMPAR and NMDAR transmission. However, further investigations should be conducted in order to conclude about the contribution of these mutants in the disease pathology.

\subsection{CDK5 phosphorylation mutants of PSD-95 regulate the developmental NMDAR subunit switch}

Until now, PSD-95 was suggested to be involved in the developmental NMDAR subunit switch in different brain regions. This hypothesis was supported by the experiments indicating higher levels of NR2B containing NMDA receptors in PSD-95 knockout animals (Béïque et al., 2006; Elias et al., 2008) and also by the correlation of the increased PSD-95 protein levels with the synchronous elevation in the synaptic GluN2A-containing NMDAR levels (Sans et al., 2000; Losi et al., 2003b; Bellone and Nicoll, 2007). However, there is no exact mechanism known how PSD-95 mediates such a developmental subunit switch at synapses. In this project, I tested the involvement of PSD-95 in the NMDAR subunit switch of visual cortex layer 2/3 neurons, which takes place upon eye opening.

First, I could prove that PSD-95 indeed is involved in the developmental NMDAR subunit switch in visual cortex layer 2/3 neurons. Both PSD-95 knockout neurons and neurons expressing shRNA against endogenous PSD-95 could not establish the NMDAR subunit switch (Figure 30, Figure 31). Recordings from the neurons expressing the shRNA against endogenous PSD-95 demonstrated the importance of PSD-95 in this process in a cell-autonomous manner which is excludes the changes in a 
network level due to PSD-95 deletion. Second, I could show that the phosphomimicking mutant of PSD-95 prevents this switch, even though the first visual stimulus is received (Figure 32). These results suggest a mechanism where PSD-95 mediates the NMDA receptor subunit switch in a CDK5 phosphorylation state-dependent manner.

Recently it was shown that CKII plays a role in the NMDAR subunit switch of hippocampal neurons. CKII levels were shown to increase in hippocampus during the critical period. The increase in CKII levels were accompanied by a decrease in GluN2B-containing receptors due to the phosphorylation of GluN2B subunit followed by their endocytosis (Sanz-Clemente et al., 2010). Interestingly, CKII was shown to inhibit CDK5 activity in neurons (Lim et al., 2004). It is possible that increases in the CKII levels reduces the CDK5 activity thus the phosphorylation on PSD-95 and allowing the subunit switch to take place. Therefore, keeping PSD-95 in the phosphorylated configuration might interfere with the mechanism to mediate the switch.

The regulation of the NMDAR subunit switch within the critical period has several important consequences. First, the composition of NMDA receptors was indicated to be crucial for the synaptogenesis. GluN2A overexpression in hippocampus was shown to reduce synapse numbers, volume and dynamics of the spines, whereas overexpression of GluN2B subunit maintained increased mobility of the spines (Gambrill and Barria, 2011). Thus, delaying the subunit switch would postpone the stabilization of the neuronal network thus alter its activity. Second, the composition of NMDA receptor subunits is an important determinant for different plasticity forms, such as ocular dominant plasticity and metaplasticity. Cho et al., 2009 demonstrated that the ratio of GluN2A/GluN2B in visual cortex is important for enabling the activity-dependent weakening of the synapses upon monocular deprivation. In addition, the function of GluN2A- and GluN2B-containing receptors was shown to mediate metaplasticity by lowering or raising the thresholds for LTP and LTD (Yang et al., 2012). Besides the molecular level, the absence of GluN2A in the synapses demonstrates behavioral and learning deficits, such as impaired spatial learning memory, decreased anxiety and depression-like behavior (Boyce-Rustay and Holmes, 2006; Bannerman et al., 2008).

With respect to all important consequences of NMDA receptor subunit composition, CDK5 phosphorylation mutants of PSD-95 yet present another cue how the subunit 
switch might be regulated. In order to understand whether the mechanism involving the phospho-mimicking mutant of PSD-95 requires Src kinase activity and GluN2B phosphorylation, further experiments should be conducted by manipulating endogenous activity of CDK5 and Src kinase, as well as by checking the phosphorylation state of different AMPAR and NMDAR subunits.

\subsection{Minimal PSD-95 to regulate basal synaptic transmission in CA1 region of hippocampus}

The function of PSD-95 strongly depends on its N-terminus. Two cysteine residues, $\mathrm{C} 3$ and $\mathrm{C5}$, which are located at the N-terminus of PSD-95, were shown to undergo palmitoylation. This modification is required for the multimerization of PSD-95 thus its proper membrane tethering in order to regulate the AMPA receptor function in CA1 region hippocampal neurons (Schnell et al., 2002; Xu et al., 2008). At the same time, the expression of a PSD-95 mutant containing only its first two PDZ domains in addition to the intact $\mathrm{N}$-terminus, failed to rescue AMPAR responses in the absence of the endogenous PSD-95 (Xu et al., 2008). However, it could enhance the AMPAR transmission in the presence of endogenous PSD-95 (Schnell et al., 2002). Moreover, a PSD-95 mutant lacking the GK and SH3 domains was shown to be not functional when endogenous PSD-95 was knocked-down (Xu et al., 2008). These data suggested that GK and SH3 domains are necessary for mediating the basal synaptic transmission in CA1 region hippocampal neurons, but the contribution of each domain is not clear.

With this project to understand the minimal form of PSD-95 for the regulation of the basal synaptic transmission in hippocampus, we examined the roles of PDZ3, GK and SH3 domains separately.

Expression of a SH3 deletion mutant of PSD-95 in combination with the shRNA against endogenous PSD-95 enhanced the basal synaptic transmission properties of rat CA1 region hippocampal neurons, implying that the $\mathrm{SH} 3$ domain is dispensable for the purpose of regulating the basal synaptic transmission (Figure 34). This result is supported by a recent study by Jo et al., 2010 showing a similar increase in AMPAR responses of neurons expressing the SH3 deletion mutant of PSD-95 when combined with the knock-down of endogenous PSD-95. In addition to being involved in intra- 
molecular interactions, SH3 binds to different proteins such as hippocalcin and Pyk2 (Seabold et al., 2003; Jo et al., 2010). These proteins are indicated with their role in hippocampal long-term plasticity (Bartos et al., 2010; Jo et al., 2010). Thus, the results from the rat slice culture recordings rat suggest that $\mathrm{SH} 3$ might be not required for the regulation of basal synaptic transmission but might be necessary for the processes in hippocampal long-term plasticity.

Deletion of the GK domain from PSD-95 or expression of PDZ1/2-GK could only partially rescue the knock-down effect of endogenous PSD-95 in CA1 region neurons of rat hippocampus (Figure 34, Figure 35). This partial rescue was not due to a functional impairment of the PSD-95 $\Delta \mathrm{GK}$ construct, as overexpression of the mutant could significantly increase the AMPAR transmission of the infected neurons (Figure 35). We asked whether this partial rescue is due to a partial knock-down effect in the rat slice culture system, that's why we tested the effect of the PSD-95 $\Delta \mathrm{GK}$ mutant on wild-type and PSD-95 knockout mouse background. Expression of the same mutant on these backgrounds showed a significant increase in AMPAR transmission similar to the full-length PSD-95 expression (Figure 36, Figure 38). The result from the wild-type and PSD-95 knockout mice slices suggested that the GK domain is not crucial for mediating the basal synaptic transmission in CA1 region mouse hippocampal neurons. This result was in consistency with the data published by Jo et al., 2010, showing that the replacement of endogenous PSD-95 by the mutant form lacking the GK domain still enhances the AMPAR transmission of hippocampal neurons. GK domain is known to interact with several proteins, some of which are Shank (Naisbitt et al., 1999), MAP1 (Reese et al., 2007), SPAR (Pak et al., 2001) and GKAP/SAPAP (Kim et al., 1997; Takeuchi et al., 1997; Ehlers, 2003). Shank and GKAP/SAPAP are important to attach PSD-95 to the cytoskeleton of the postsynaptic density thus regulate the transmission strength of the excitatory synapses (Valtschanoff and Weinberg, 2001; Hayashi et al., 2009). This data suggests that using its interaction partners a minimal form of PSD-95 composing of the first two PDZ domains and the GK domain is functional to regulate the AMPAR transmission in PSD-95 knockout hippocampal neurons.

We also wanted to test the involvement of PDZ3 domain in the regulation of basal synaptic transmission in CA1 region hippocampal neurons. For this purpose, we 
replaced the endogenous PSD-95 with a mutant form lacking the PDZ3 domain in rat hippocampal slices. Recordings showed a partial rescue by the mutant, similar to the GK domain-lacking mutant (Figure 34). Additionally, expression of the PDZ3 lacking PSD-95 in the PSD-95 knockout background enhanced the AMPAR responses of the infected neurons significantly (Figure 38). This observation indicates that the PDZ3 domain of PSD-95 is not necessary for the regulation of the basal synaptic transmission in CA1 region neurons of PSD-95 knockout animals. PDZ3 domain of PSD-95 binds to proteins like CRIPT, neuroligins and Frizzled which are important for the cytoskeletal interaction, cross-synaptic adhesions, cell polarization and synaptogenesis (Irie et al., 1997; Niethammer et al., 1998; Wheeler et al., 2011). Regarding the results gathered from the PDZ3 domain-lacking mutant expression in the PSD-95 knockout slices, these interactions do not seem to be crucial for the regulation of basal synaptic transmission in CA1 region neurons of knockout animals.

Lastly, since the PDZ3 and GK domains in the PSD-95 knockout background occurred to be dispensable for the regulation of the basal synaptic transmission in CA1 region neurons, we analyzed whether a PSD-95 form composing of the first two PDZ domains as well as the $\mathrm{SH} 3$ domain would be adequate to regulate the basal synaptic transmission. Interestingly, this mutant form of PSD-95 could rescue the AMPAR transmission in PSD-95 knockout neurons in CA1 region of hippocampus (Figure 42).

Herewith, we suggest two minimal PSD-95 forms expressing the first two PDZ domains and either of the GK or SH3 domain (PDZ1/2-GK and PDZ1/2-SH3) for the regulation of basal synaptic transmission in PSD-95 knockout background. Both of the mutants enhance the AMPAR transmission of CA1 region neurons in PSD-95 knockout slices in a similar way to the full-length PSD95. Since DLG-MAGUKs were shown to interact with each other through their SH3 and GK domains (McGee et al., 2001a), it is possible that having only the GK or the SH3 domain in addition to the first two PDZ domains is enough for PSD-95 to create intermolecular connections hence regulate the basal synaptic transmission in the CA1 region neurons of PSD-95 knockout animals. 


\subsection{Compensation mechanism between the MAGUK members regarding the basal synaptic transmission in CA1 region neurons}

Recordings obtained from the rat and PSD-95 knockout mouse slices demonstrated a difference in the effects of the PSD-95 $\Delta \mathrm{GK}$, PSD-95 $\Delta$ PDZ3 and PDZ1/2-GK mutants on the AMPAR transmission of CA1 region neurons. One of the reasons which might cause this difference is a potential compensation mechanisms among different members of the MAGUK family (Elias et al., 2006; Cuthbert et al., 2007). DLG-MAGUKs were shown to interact with each other via their GK and SH3 domains (McGee et al., 2001a). To test the possibility that this could be the case in the PSD-95 knockout slices, we quantified the amount of PSD-93, SAP102 and SAP97 in the PSD of PSD-95 knockout animal brain homogenates (Figure 39). We observed a significant increase in the levels of SAP102 and SAP97 but not in PSD-93 levels in the PSD-95 knockout animals. In addition, when we tested the levels of the MAGUKs in the PSD of PSD-93 knockout animals, there was no significant increase in PSD-95, SAP102 and SAP97 levels. This implies that the compensation mechanism observed among the MAGUKs is specific for the PSD-95 knockout animals. At the same time, the difference in the protein levels of MAGUKs suggests a difference in the maturational state of rat and PSD-95 knockout mouse slices at the time point where the experiments were performed. Rat hippocampal slices are prepared when PSD-95 expression is present, thus the development takes place without an interruption, whereas PSD-95 knockout mouse slices cannot reach a mature state due to the lack of PSD-95 and keep higher levels of SAP102 and SAP97 which traps them in an immature state. This concept is supported by the findings of Snyder et al., 2009 showing that the hippocampal neurons in rat mature faster than the neurons in mouse.

Hence, the compensation in the levels of DLG-MAGUKs of the PSD-95 knockout animals and the maturation state of the knockout slices can account for the differences observed with the electrophysiological recordings. 


\subsection{Molecular interplay between PSD-95 and SAP102 in}

\section{CA1 region neurons of PSD-95 knockout animals}

After observing the increased levels of SAP102 and SAP97 in PSD-95 knockout animals, we tested the constructs in combination with the shRNA against SAP102. SAP102 was shown to be important for the basal synaptic transmission in early development and the knock-down of SAP102 had different impacts depending on the developmental stage (Elias et al., 2008). In the presence of high SAP102 levels, all mutants of PSD-95 (PSD-95 $\Delta \mathrm{GK}$, PSD-95 $\Delta$ PDZ3, PSD-95 $\Delta \mathrm{SH} 3$ (data now shown, see (Bonnet, 2011), PDZ1/2-GK and PDZ1/2-SH3) showed a significant increase in AMPAR responses of CA1 region neurons (Figure 38, Figure 41, Figure 42). However, knocking-down SAP102 eliminated the increase in AMPAR transmission observed with the PSD-95 $\Delta \mathrm{GK}$, PSD-95 $\Delta$ PDZ3 and PDZ1/2-SH3 mutants (Figure 40, Figure 41, Figure 42). At the same time we tested the PSD-95 $\Delta \mathrm{GK}$ mutant in combination with the shRNA against SAP97. PSD-95 $\Delta \mathrm{GK}$ could still enhance the AMPAR transmission of the infected neurons, when SAP97 was knocked-down (Figure 40). These results suggest that PSD-95 $\Delta \mathrm{GK}$, PSD-95 $\Delta$ PDZ3 and PDZ1/2-SH3 mutants require increased levels of SAP102 to be functional in PSD-95 knockout background and for PSD-95 $\Delta \mathrm{GK}$ function SAP97 is not a requirement.

On the other hand, knock-down of SAP102 did not affect the significant increases in AMPAR transmission mediated by the PSD-95 $\Delta \mathrm{SH} 3$ (data now shown, see Bonnet, 2011) and PDZ1/2-GK mutants (Figure 41), implying that these mutants do not require SAP102 for the enhancement of AMPAR responses in the PSD-95 knockout background.

An interesting conclusion regarding these results was that SAP102 knock-down could abolish the AMPAR enhancement only if the PSD-95 mutant contained the SH3 domain. The mutants which did not contain SH3 but the GK domain maintained the significant increase in AMPAR responses, even if SAP102 was knocked-down. A potential candidate to support the function of these mutants might be SAP97. However, for such a conclusion, PSD-95 $\Delta \mathrm{SH} 3$ and PDZ1/2-GK mutants should be expressed in combination with shRNA against SAP97 or in a PSD-95/SAP97 knockout background. 
If SAP97 is not required, the enhancement in AMPAR responses might be due to the intrinsic properties of the mutants.

The data until now shows that the SH3 domain is required to link SAP102 to PSD95 either directly or through AMPAR and NMDAR complexes. GK domain, on the other hand, might be necessary for PSD-95 to be linked to SAP97. Such a mechanism would suggest a domain-specific interaction among DLG-MAGUKs, which will increase the complexity of the MAGUK function field.

\subsection{NMDAR transmission of minimal PSD-95 constructs}

During the recordings, some minor but significant changes were observed in the NMDAR transmission of certain minimal PSD-95 mutant constructs. In rat slice culture, wild-type PSD-95 and PSD-95 $\Delta \mathrm{SH} 3$ replacement constructs enhanced both AMPAR and NMDAR transmission (Figure 33, Figure 34). In addition, sh102 + PSD-95 $\Delta G K$ and PDZ1/2-SH3 expression in the PSD-95 knockout mouse slices showed a significant increase (Figure 40, Figure 42), whereas sh102 + PSD-95 $\Delta$ PDZ3 construct demonstrated a significant decrease in NMDAR transmission of the infected neurons (Figure 41).

It is known that PSD-95 is involved in the trafficking and function of AMPA receptors specifically (Schnell et al., 2002; Ehrlich and Malinow, 2004; Schlüter et al., 2006; Xu et al., 2008). Nevertheless, some groups demonstrated changes on NMDAR transmission upon PSD-95 manipulation, which might be due to the changes in PSD-95 levels as well as the number of neurons affected (Futai et al., 2007; Kim et al., 2007).

PDZ3 domain was shown to interact with neuroligin, an important protein contributing to the synapse formation and stability (Irie et al., 1997; Washbourne et al., 2004). The decrease observed in the NMDAR transmission of the neurons expressing sh102 + PSD-95 $\triangle$ PDZ3 might be due to an interruption in the PDZ3 and neuroligin binding. This might result in a decreased number of synapses. However, expression of sh $102+$ PDZ1/2-SH3, which also lacks the PDZ3 domain, exhibited normal levels of NMDAR transmission, making it unlikely to have decreased synapse number in the absence of PDZ3 domain. Thus, even though small changes in NMDAR transmission are observed, PSD-95 domains mainly exert their effect on AMPAR transmission. 


\subsection{Conclusions and Outlook}

With the presented studies, we could show that PSD-95 regulates the basal synaptic transmission in CA1 region neurons of rat hippocampus in a CDK5 phosphorylation state-dependent manner. This regulation requires the activity of Src kinases and the involvement of GluN2B-containing NMDA receptors.

Furthermore, we could demonstrate that PSD-95 mediates the developmental NMDA receptor subunit switch in layer $2 / 3$ neurons of mouse visual cortex. Expression of a PSD-95 form mimicking the CDK5 phosphorylation state prevents the switch from taking place, pointing to the potential involvement of CDK5 in the regulation of NMDAR subunit switch.

Lastly, we revealed two important domains of PSD-95 (SH3 and GK) for mediating the basal synaptic transmission in CA1 region neurons of PSD-95 knockout mouse hippocampus. Presence of these domains is required for specific molecular interactions among the DLG-MAGUK members. SH3 domain of PSD-95 is necessary for linking PSD-95 to SAP102, whereas GK domain in the absence of SH3 domain regulates the AMPAR transmission in a SAP102 independent way. Furthermore, we could show that there is a difference in the maturational state of rat and PSD-95 knockout mouse hippocampal neurons, which may count for the differences in the electrophysiological results obtained from the same constructs.

Nevertheless, there are still open questions to address to create a better understanding about the mechanisms regulating the above-mentioned processes. These questions need to be addresses by:

- Manipulating the endogenous CDK5 and Src kinase activity to validate the effects of the CDK5 phosphorylation mutants of PSD-95 as well as to analyze the contribution of the kinases to the basal synaptic transmission in CA1 region neurons

- Quantifying the phosphorylation levels of AMPAR and NMDAR subunits in the CDK5 phosphorylation mutant expressing and control neurons

- Manipulating the endogenous CDK5, Src kinase and CKII activity to understand the mechanism of the developmental NMDAR subunit switch mediated by the CDK5 phosphorylation mutants of PSD-95 
- Analyzing the requirement of GK domain in the context of PSD-95 and SAP97 interaction. 


\section{References}

Al-Hallaq RA, Conrads TP, Veenstra TD, Wenthold RJ (2007) NMDA di-heteromeric receptor populations and associated proteins in rat hippocampus. The Journal of neuroscience : the official journal of the Society for Neuroscience 27:8334-8343.

Andrasfalvy BK, Magee JC (2001) Distance-dependent increase in AMPA receptor number in the dendrites of adult hippocampal CA1 pyramidal neurons. The Journal of neuroscience : the official journal of the Society for Neuroscience 21:9151-9159.

Arendt KL, Sarti F, Chen L (2013) Chronic inactivation of a neural circuit enhances LTP by inducing silent synapse formation. The Journal of neuroscience : the official journal of the Society for Neuroscience 33:2087-2096.

Bannerman DM, Niewoehner B, Lyon L, Romberg C, Schmitt WB, Taylor A, Sanderson DJ, Cottam J, Sprengel R, Seeburg PH, Köhr G, Rawlins JNP (2008) NMDA receptor subunit NR2A is required for rapidly acquired spatial working memory but not incremental spatial reference memory. The Journal of neuroscience : the official journal of the Society for Neuroscience 28:3623-3630.

Barria A, Derkach V, Soderling T (1997) Identification of the Ca2+/calmodulin-dependent protein kinase II regulatory phosphorylation site in the alpha-amino-3-hydroxyl-5-methyl4-isoxazole-propionate-type glutamate receptor. The Journal of biological chemistry 272:32727-32730.

Barria A, Malinow R (2005) NMDA receptor subunit composition controls synaptic plasticity by regulating binding to CaMKII. Neuron 48:289-301.

Bartos JA, Ulrich JD, Li H, Beazely MA, Chen Y, Macdonald JF, Hell JW (2010) Postsynaptic clustering and activation of Pyk2 by PSD-95. The Journal of neuroscience : the official journal of the Society for Neuroscience 30:449-463.

Bashir ZI, Alford S, Davies SN, Randall AD, Collingridge GL (1991) Long-term potentiation of NMDA receptor-mediated synaptic transmission in the hippocampus. Nature 349:156158.

Bayer KU, De Koninck P, Leonard AS, Hell JW, Schulman H (2001) Interaction with the NMDA receptor locks CaMKII in an active conformation. Nature 411:801-805.

Bellone C, Mameli M, Lüscher C (2011) In utero exposure to cocaine delays postnatal synaptic maturation of glutamatergic transmission in the VTA. Nature neuroscience 14:14391446.

Bellone C, Nicoll RA (2007) Rapid Bidirectional Switching of Synaptic NMDA Receptors. Neuron 55:779-785.

Bennett MV., Zukin RS (2004) Electrical Coupling and Neuronal Synchronization in the Mammalian Brain. Neuron 41:495-511. 
Benquet P, Gee CE, Gerber U (2002) Two distinct signaling pathways upregulate NMDA receptor responses via two distinct metabotropic glutamate receptor subtypes. The Journal of neuroscience : the official journal of the Society for Neuroscience 22:9679-9686.

Berretta N, Berton F, Bianchi R, Brunelli M, Capogna M, Francesconi W (1991) Long-term Potentiation of NMDA Receptor-mediated EPSP in Guinea-pig Hippocampal Slices. The European journal of neuroscience 3:850-854.

Bhatt JM, Prakash A, Suryavanshi PS, Dravid SM (2013) Effect of Ifenprodil on GluN1/GluN2B N-Methyl-D-aspartate Receptor Gating. Molecular pharmacology 83:921.

Bhattacharyya S, Biou V, Xu W, Schluter O, Malenka RC (2009) A critical role for PSD95/AKAP interactions in endocytosis of synaptic AMPA receptors. Nat Neurosci 12:172181.

Bianchetta MJ, Lam TT, Jones SN, Morabito MA (2011) Cyclin-Dependent Kinase 5 Regulates PSD-95 Ubiquitination in Neurons. The Journal of Neuroscience 31:12029-12035.

Birnboim HC, Doly J (1979) A rapid alkaline extraction procedure for screening recombinant plasmid DNA. Nucleic acids research 7:1513-1523.

Bliss T V, Lomo T (1973) Long-lasting potentiation of synaptic transmission in the dentate area of the anaesthetized rabbit following stimulation of the perforant path. The Journal of physiology 232:331-356.

Boehm J, Kang M-G, Johnson RC, Esteban J, Huganir RL, Malinow R (2006) Synaptic incorporation of AMPA receptors during LTP is controlled by a PKC phosphorylation site on GluR1. Neuron 51:213-225.

Bonnet SAD (2011) Role of the Different Domains of PSD-95 in Basal Synaptic Transmission.

Boyce-Rustay JM, Holmes A (2006) Genetic inactivation of the NMDA receptor NR2A subunit has anxiolytic- and antidepressant-like effects in mice.

Neuropsychopharmacology : official publication of the American College of Neuropsychopharmacology 31:2405-2414.

Brenman JE, Christopherson KS, Craven SE, McGee AW, Bredt DS (1996) Cloning and characterization of postsynaptic density 93 , a nitric oxide synthase interacting protein. The Journal of neuroscience : the official journal of the Society for Neuroscience 16:74077415 .

Burnashev N, Monyer H, Seeburg PH, Sakmann B (1992) Divalent ion permeability of AMPA receptor channels is dominated by the edited form of a single subunit. Neuron 8:189-198.

Béïque J-C, Andrade R (2003) PSD-95 regulates synaptic transmission and plasticity in rat cerebral cortex. The Journal of physiology 546:859-867. 
Béïque J-C, Lin D-T, Kang M-G, Aizawa H, Takamiya K, Huganir RL (2006) Synapse-specific regulation of AMPA receptor function by PSD-95. Proceedings of the National Academy of Sciences 103:19535-19540.

Cabantous S, Terwilliger TC, Waldo GS (2005) Protein tagging and detection with engineered self-assembling fragments of green fluorescent protein. Nature biotechnology 23:102107.

Carlisle HJ, Fink AE, Grant SGN, O’Dell TJ (2008) Opposing effects of PSD-93 and PSD-95 on long-term potentiation and spike timing-dependent plasticity. The Journal of Physiology 586:5885-5900.

Caruana G, Bernstein A (2001) Craniofacial dysmorphogenesis including cleft palate in mice with an insertional mutation in the discs large gene. Molecular and cellular biology $21: 1475-1483$.

Carvalho AL, Kameyama K, Huganir RL (1999) Characterization of phosphorylation sites on the glutamate receptor 4 subunit of the AMPA receptors. The Journal of neuroscience : the official journal of the Society for Neuroscience 19:4748-4754.

Casimiro TM, Sossa KG, Uzunova G, Beattie JB, Marsden KC, Carroll RC (2011) mGluR and NMDAR activation internalize distinct populations of AMPARs. Molecular and cellular neurosciences 48:161-170.

Chae T, Kwon YT, Bronson R, Dikkes P, Li E, Tsai LH (1997) Mice lacking p35, a neuronal specific activator of Cdk5, display cortical lamination defects, seizures, and adult lethality. Neuron 18:29-42.

Chang EH, Savage MJ, Flood DG, Thomas JM, Levy RB, Mahadomrongkul V, Shirao T, Aoki C, Huerta PT (2006) AMPA receptor downscaling at the onset of Alzheimer's disease pathology in double knockin mice. Proceedings of the National Academy of Sciences of the United States of America 103:3410-3415.

Chen B-S, Roche KW (2007) Regulation of NMDA receptors by phosphorylation. Neuropharmacology 53:362-368.

Chen L, Chetkovich DM, Petralia RS, Sweeney NT, Kawasaki Y, Wenthold RJ, Bredt DS, Nicoll RA (2000) Stargazin regulates synaptic targeting of AMPA receptors by two distinct mechanisms. Nature 408:936-943.

Chetkovich DM, Bunn RC, Kuo S-H, Kawasaki Y, Kohwi M, Bredt DS (2002) Postsynaptic Targeting of Alternative Postsynaptic Density-95 Isoforms by Distinct Mechanisms. J Neurosci 22:6415-6425.

Cheung ZH, Fu AKY, Ip NY (2006) Synaptic Roles of Cdk5: Implications in Higher Cognitive Functions and Neurodegenerative Diseases. Neuron 50:13-18.

Cho KKA, Khibnik L, Philpot BD, Bear MF (2009) The ratio of NR2A/B NMDA receptor subunits determines the qualities of ocular dominance plasticity in visual cortex. 
Proceedings of the National Academy of Sciences of the United States of America 106:5377-5382.

Cho KO, Hunt CA, Kennedy MB (1992) The rat brain postsynaptic density fraction contains a homolog of the Drosophila discs-large tumor suppressor protein. Neuron 9:929-942.

Chung HJ, Huang YH, Lau L-F, Huganir RL (2004) Regulation of the NMDA receptor complex and trafficking by activity-dependent phosphorylation of the NR2B subunit PDZ ligand. The Journal of neuroscience : the official journal of the Society for Neuroscience 24:10248-10259.

Colledge M, Snyder EM, Crozier RA, Soderling JA, Jin Y, Langeberg LK, Lu H, Bear MF, Scott JD (2003) Ubiquitination Regulates PSD-95 Degradation and AMPA Receptor Surface Expression. Neuron 40:595-607.

Costa L, Santangelo F, Li Volsi G, Ciranna L (2009) Modulation of AMPA receptor-mediated ion current by pituitary adenylate cyclase-activating polypeptide (PACAP) in CA1 pyramidal neurons from rat hippocampus. Hippocampus 19:99-109.

Craven SE, El-Husseini AE, Bredt DS (1999) Synaptic targeting of the postsynaptic density protein PSD-95 mediated by lipid and protein motifs. Neuron 22:497-509.

Crump FT, Dillman KS, Craig AM (2001) cAMP-dependent protein kinase mediates activityregulated synaptic targeting of NMDA receptors. The Journal of neuroscience : the official journal of the Society for Neuroscience 21:5079-5088.

Cruz JC, Tsai L-H (2004) Cdk5 deregulation in the pathogenesis of Alzheimer's disease. Trends in Molecular Medicine 10:452-458.

Cull-Candy SG, Leszkiewicz DN (2004) Role of distinct NMDA receptor subtypes at central synapses. Science's STKE : signal transduction knowledge environment 2004:re16.

Cuthbert PC, Stanford LE, Coba MP, Ainge JA, Fink AE, Opazo P, Delgado JY, Komiyama NH, O'Dell TJ, Grant SGN (2007) Synapse-associated protein 102/dlgh3 couples the NMDA receptor to specific plasticity pathways and learning strategies. The Journal of neuroscience : the official journal of the Society for Neuroscience 27:2673-2682.

Delgado JY, Coba M, Anderson CNG, Thompson KR, Gray EE, Heusner CL, Martin KC, Grant SGN, O’Dell TJ (2007) NMDA receptor activation dephosphorylates AMPA receptor glutamate receptor 1 subunits at threonine 840 . The Journal of neuroscience : the official journal of the Society for Neuroscience 27:13210-13221.

Dickenson AH, Sullivan AF (1991) NMDA receptors and central hyperalgesic states. Pain 46:344-346.

Dingledine R, Borges K, Bowie D, Traynelis SF (1999) The glutamate receptor ion channels. Pharmacological reviews 51:7-61. 
Dumas TC (2005) Developmental regulation of cognitive abilities: modified composition of a molecular switch turns on associative learning. Progress in neurobiology 76:189-211.

Dunah AW, Wang Y, Yasuda RP, Kameyama K, Huganir RL, Wolfe BB, Standaert DG (2000) Alterations in subunit expression, composition, and phosphorylation of striatal N-methylD-aspartate glutamate receptors in a rat 6-hydroxydopamine model of Parkinson's disease. Molecular pharmacology 57:342-352.

Ehlers MD (2003) Activity level controls postsynaptic composition and signaling via the ubiquitin-proteasome system. Nature neuroscience 6:231-242.

Ehrlich I, Klein M, Rumpel S, Malinow R (2007) PSD-95 is required for activity-driven synapse stabilization. Proceedings of the National Academy of Sciences of the United States of America 104:4176-4181.

Ehrlich I, Malinow R (2004) Postsynaptic Density 95 controls AMPA Receptor Incorporation during Long-Term Potentiation and Experience-Driven Synaptic Plasticity. The Journal of Neuroscience 24:916-927.

El-Husseini AE, Craven SE, Chetkovich DM, Firestein BL, Schnell E, Aoki C, Bredt DS (2000a) Dual palmitoylation of PSD-95 mediates its vesiculotubular sorting, postsynaptic targeting, and ion channel clustering. The Journal of cell biology 148:159-172.

El-Husseini AE, Topinka JR, Lehrer-Graiwer JE, Firestein BL, Craven SE, Aoki C, Bredt DS (2000b) Ion channel clustering by membrane-associated guanylate kinases. Differential regulation by $\mathrm{N}$-terminal lipid and metal binding motifs. The Journal of biological chemistry 275:23904-23910.

El-Husseini AE-D, Schnell E, Chetkovich DM, Nicoll RA, Bredt DS (2000c) PSD-95 Involvement in Maturation of Excitatory Synapses. Science 290:1364-1368.

Elias GM, Elias LAB, Apostolides PF, Kriegstein AR, Nicoll RA (2008) Differential trafficking of AMPA and NMDA receptors by SAP102 and PSD-95 underlies synapse development. Proceedings of the National Academy of Sciences 105:20953-20958.

Elias GM, Funke L, Stein V, Grant SG, Bredt DS, Nicoll RA (2006) Synapse-Specific and Developmentally Regulated Targeting of AMPA Receptors by a Family of MAGUK Scaffolding Proteins. Neuron 52:307-320.

Elias GM, Nicoll RA (2007) Synaptic trafficking of glutamate receptors by MAGUK scaffolding proteins. Trends in Cell Biology 17:343-352.

Elsberg CA (1931) Dr. Edwin Smith surgical papyrus and the diagnosis and treatment of injuries to the skull and spine 5000 years ago.

Erreger K, Dravid SM, Banke TG, Wyllie DJA, Traynelis SF (2005) Subunit-specific gating controls rat NR1/NR2A and NR1/NR2B NMDA channel kinetics and synaptic signalling profiles. The Journal of physiology 563:345-358. 
Fagiolini M, Katagiri H, Miyamoto H, Mori H, Grant SGN, Mishina M, Hensch TK (2003) Separable features of visual cortical plasticity revealed by N-methyl-d-aspartate receptor 2A signaling. Proceedings of the National Academy of Sciences 100:2854-2859.

Fang W-Q, Ip JPK, Li R, Ng YP, Lin S-C, Chen Y, Fu AKY, Ip NY (2011) Cdk5-mediated phosphorylation of Axin directs axon formation during cerebral cortex development. The Journal of neuroscience : the official journal of the Society for Neuroscience 31:1361313624.

Feng W, Zhang M (2009) Organization and dynamics of PDZ-domain-related supramodules in the postsynaptic density. Nat Rev Neurosci 10:87-99.

Flint AC, Maisch US, Weishaupt JH, Kriegstein AR, Monyer H (1997) NR2A subunit expression shortens NMDA receptor synaptic currents in developing neocortex. The Journal of neuroscience : the official journal of the Society for Neuroscience 17:24692476.

Foster KA, McLaughlin N, Edbauer D, Phillips M, Bolton A, Constantine-Paton M, Sheng M (2010) Distinct Roles of NR2A and NR2B Cytoplasmic Tails in Long-Term Potentiation. J Neurosci 30:2676-2685.

Fox CJ, Russell KI, Wang YT, Christie BR (2006) Contribution of NR2A and NR2B NMDA subunits to bidirectional synaptic plasticity in the hippocampus in vivo. Hippocampus 16:907-915.

Fukaya M, Kato A, Lovett C, Tonegawa S, Watanabe M (2003) Retention of NMDA receptor NR2 subunits in the lumen of endoplasmic reticulum in targeted NR1 knockout mice. Proceedings of the National Academy of Sciences of the United States of America 100:4855-4860.

Funahashi R, Maruyama T, Yoshimura Y, Komatsu Y (2013) Silent synapses persist into adulthood in layer $2 / 3$ pyramidal neurons of visual cortex in dark-reared mice. Journal of neurophysiology.

Futai K, Kim MJ, Hashikawa T, Scheiffele P, Sheng M, Hayashi Y (2007) Retrograde modulation of presynaptic release probability through signaling mediated by PSD-95neuroligin. Nature neuroscience 10:186-195.

Gambrill AC, Barria A (2011) NMDA receptor subunit composition controls synaptogenesis and synapse stabilization. Proceedings of the National Academy of Sciences.

Gardner SM, Takamiya K, Xia J, Suh J-G, Johnson R, Yu S, Huganir RL (2005) Calciumpermeable AMPA receptor plasticity is mediated by subunit-specific interactions with PICK1 and NSF. Neuron 45:903-915.

Gardoni F, Bellone C, Cattabeni F, Di Luca M (2001) Protein kinase C activation modulates alpha-calmodulin kinase II binding to NR2A subunit of N-methyl-D-aspartate receptor complex. The Journal of biological chemistry 276:7609-7613. 
Gardoni F, Marcello E, Di Luca M (2009) Postsynaptic density-membrane associated guanylate kinase proteins (PSD-MAGUKs) and their role in CNS disorders. Neuroscience 158:324333.

Gardoni F, Polli F, Cattabeni F, Di Luca M (2006) Calcium-calmodulin-dependent protein kinase II phosphorylation modulates PSD-95 binding to NMDA receptors. Blackwell Publishing Ltd.

Gaspar PA, Bustamante ML, Silva H, Aboitiz F (2009) Molecular mechanisms underlying glutamatergic dysfunction in schizophrenia: therapeutic implications. Journal of neurochemistry 111:891-900.

Goodenough DA, Goliger JA, Paul DL (1996) Connexins, connexons, and intercellular communication. Annual review of biochemistry 65:475-502.

Graham DRM, Garnham CP, Fu Q, Robbins J, Van Eyk JE (2005) Improvements in twodimensional gel electrophoresis by utilizing a low cost "in-house" neutral $\mathrm{pH}$ sodium dodecyl sulfate-polyacrylamide gel electrophoresis system. Proteomics 5:2309-2314.

Graham FL, Smiley J, Russell WC, Nairn R (1977) Characteristics of a human cell line transformed by DNA from human adenovirus type 5. The Journal of general virology $36: 59-74$.

Grant ER, Guttmann RP, Seifert KM, Lynch DR (2001) A region of the rat N-methyl-Daspartate receptor $2 \mathrm{~A}$ subunit that is sufficient for potentiation by phorbol esters. Neuroscience letters 310:9-12.

Greenamyre JT, Porter RH (1994) Anatomy and physiology of glutamate in the CNS. Neurology 44:S7-13.

Groc L, Heine M, Cognet L, Lounis B, Choquet D (2006a) Lateral Diffusion of Excitatory Neurotransmitter Receptors During Synaptogenesis. Molecular Mechanisms of Synaptogenesis:221-232.

Groc L, Heine M, Cousins SL, Stephenson FA, Lounis B, Cognet L, Choquet D (2006b) NMDA receptor surface mobility depends on NR2A-2B subunits. Proceedings of the National Academy of Sciences of the United States of America 103:18769-18774.

Grooms SY, Noh K-M, Regis R, Bassell GJ, Bryan MK, Carroll RC, Zukin RS (2006) Activity bidirectionally regulates AMPA receptor mRNA abundance in dendrites of hippocampal neurons. The Journal of neuroscience : the official journal of the Society for Neuroscience 26:8339-8351.

Halt AR, Dallapiazza RF, Zhou Y, Stein IS, Qian H, Juntti S, Wojcik S, Brose N, Silva AJ, Hell JW (2012) CaMKII binding to GluN2B is critical during memory consolidation. The EMBO journal 31:1203-1216. 
Hayashi MK, Tang C, Verpelli C, Narayanan R, Stearns MH, Xu R-M, Li H, Sala C, Hayashi Y (2009) The postsynaptic density proteins Homer and Shank form a polymeric network structure. Cell 137:159-171.

Hayashi T, Huganir RL (2004) Tyrosine phosphorylation and regulation of the AMPA receptor by SRC family tyrosine kinases. The Journal of neuroscience : the official journal of the Society for Neuroscience 24:6152-6160.

Hayashi Y, Shi SH, Esteban JA, Piccini A, Poncer JC, Malinow R (2000) Driving AMPA receptors into synapses by LTP and CaMKII: requirement for GluR1 and PDZ domain interaction. Science (New York, NY) 287:2262-2267.

Hebb DO (1949) The organization of behavior. New York: Wiley \& Sons.

Henson MA, Roberts AC, Pérez-Otaño I, Philpot BD (2010) Influence of the NR3A subunit on NMDA receptor functions. Progress in neurobiology 91:23-37.

Hestrin S (1992) Developmental regulation of NMDA receptor-mediated synaptic currents at a central synapse. Nature 357:686-689.

Hollmann M, Heinemann S (1994) Cloned glutamate receptors. Annual review of neuroscience 17:31-108.

Howard MA, Elias GM, Elias LAB, Swat W, Nicoll RA (2010) The role of SAP97 in synaptic glutamate receptor dynamics. Proceedings of the National Academy of Sciences of the United States of America 107:3805-3810.

Hsueh YP, Sheng M (1999) Requirement of N-terminal cysteines of PSD-95 for PSD-95 multimerization and ternary complex formation, but not for binding to potassium channel Kv1.4. The Journal of biological chemistry 274:532-536.

Huettner JE, Baughman RW (1986) Primary culture of identified neurons from the visual cortex of postnatal rats. The Journal of neuroscience : the official journal of the Society for Neuroscience 6:3044-3060.

Humbert S, Lanier LM, Tsai LH (2000) Synaptic localization of p39, a neuronal activator of cdk5. Neuroreport 11:2213-2216.

Irie M, Hata Y, Takeuchi M, Ichtchenko K, Toyoda A, Hirao K, Takai Y, Rosahl TW, Südhof TC (1997) Binding of neuroligins to PSD-95. Science (New York, NY) 277:1511-1515.

Jaafari N, Henley JM, Hanley JG (2012) PICK1 mediates transient synaptic expression of GluA2-lacking AMPA receptors during glycine-induced AMPA receptor trafficking. The Journal of neuroscience : the official journal of the Society for Neuroscience 32:1161811630.

Jo J, Son GH, Winters BL, Kim MJ, Whitcomb DJ, Dickinson BA, Lee Y-B, Futai K, Amici M, Sheng M, Collingridge GL, Cho K (2010) Muscarinic receptors induce LTD of 
NMDAR EPSCs via a mechanism involving hippocalcin, AP2 and PSD-95. Nature neuroscience 13:1216-1224.

Jones ML, Leonard JP (2005) PKC site mutations reveal differential modulation by insulin of NMDA receptors containing NR2A or NR2B subunits. Journal of neurochemistry 92:1431-1438.

Kakegawa W, Tsuzuki K, Yoshida Y, Kameyama K, Ozawa S (2004) Input- and subunitspecific AMPA receptor trafficking underlying long-term potentiation at hippocampal CA3 synapses. The European journal of neuroscience 20:101-110.

Kalia L V, Gingrich JR, Salter MW (2004) Src in synaptic transmission and plasticity. Oncogene 23:8007-8016.

Kalia L V, Pitcher GM, Pelkey KA, Salter MW (2006) PSD-95 is a negative regulator of the tyrosine kinase Src in the NMDA receptor complex. EMBO J 25:4971-4982.

Kalia L V, Salter MW (2003) Interactions between Src family protein tyrosine kinases and PSD-95. Neuropharmacology 45:720-728.

Kamp MA, Tahsim-Oglou Y, Steiger H-J, Hänggi D (2012) Traumatic brain injuries in the ancient Egypt: insights from the Edwin Smith Papyrus. Journal of neurological surgery Part A, Central European neurosurgery 73:230-237.

Kelly L, Farrant M, Cull-Candy SG (2009) Synaptic mGluR activation drives plasticity of calcium-permeable AMPA receptors. Nature neuroscience 12:593-601.

Kessels HW, Malinow R (2009) Synaptic AMPA receptor plasticity and behavior. Neuron $61: 340-350$.

Kim E, Naisbitt S, Hsueh YP, Rao A, Rothschild A, Craig AM, Sheng M (1997) GKAP, a novel synaptic protein that interacts with the guanylate kinase-like domain of the PSD95/SAP90 family of channel clustering molecules. The Journal of cell biology 136:669678.

Kim MJ, Futai K, Jo J, Hayashi Y, Cho K, Sheng M (2007) Synaptic accumulation of PSD-95 and synaptic function regulated by phosphorylation of serine-295 of PSD-95. Neuron $56: 488-502$.

Komatsu Y, Iwakiri M (1993) Long-term modification of inhibitory synaptic transmission in developing visual cortex. Neuroreport 4:907-910.

Kornau HC, Schenker LT, Kennedy MB, Seeburg PH (1995) Domain interaction between NMDA receptor subunits and the postsynaptic density protein PSD-95. Science (New York, NY) 269:1737-1740.

Krüger JM (2010a) Expression and function of PSD-93 isoforms in hippocampal synapses.

Krüger JM (2010b) Expression and function of PSD-93 isoforms in hippocampal synapses. 
Kumar SS, Bacci A, Kharazia V, Huguenard JR (2002) A developmental switch of AMPA receptor subunits in neocortical pyramidal neurons. The Journal of neuroscience : the official journal of the Society for Neuroscience 22:3005-3015.

Kwon H-B, Castillo PE (2008) Long-term potentiation selectively expressed by NMDA receptors at hippocampal mossy fiber synapses. Neuron 57:108-120.

Köhr G, Seeburg PH (1996) Subtype-specific regulation of recombinant NMDA receptorchannels by protein tyrosine kinases of the src family. The Journal of physiology 492 ( $\mathrm{Pt}$ $2: 445-452$.

Lee FJS, Xue S, Pei L, Vukusic B, Chéry N, Wang Y, Wang YT, Niznik HB, Yu X, Liu F (2002a) Dual regulation of NMDA receptor functions by direct protein-protein interactions with the dopamine D1 receptor. Cell 111:219-230.

Lee H-K, Takamiya K, He K, Song L, Huganir RL (2010) Specific roles of AMPA receptor subunit GluR1 (GluA1) phosphorylation sites in regulating synaptic plasticity in the CA1 region of hippocampus. Journal of neurophysiology 103:479-489.

Lee HK, Barbarosie M, Kameyama K, Bear MF, Huganir RL (2000) Regulation of distinct AMPA receptor phosphorylation sites during bidirectional synaptic plasticity. Nature 405:955-959.

Lee S, Fan S, Makarova O, Straight S, Margolis B (2002b) A novel and conserved proteinprotein interaction domain of mammalian Lin-2/CASK binds and recruits SAP97 to the lateral surface of epithelia. Molecular and cellular biology 22:1778-1791.

Leonard AS, Davare MA, Horne MC, Garner CC, Hell JW (1998) SAP97 is associated with the alpha-amino-3-hydroxy-5-methylisoxazole-4-propionic acid receptor GluR1 subunit. The Journal of biological chemistry 273:19518-19524.

Lewis P, Hensel M, Emerman M (1992) Human immunodeficiency virus infection of cells arrested in the cell cycle. The EMBO journal 11:3053-3058.

Li B-S, Sun M-K, Zhang L, Takahashi S, Ma W, Vinade L, Kulkarni AB, Brady RO, Pant HC (2001) Regulation of NMDA receptors by cyclin-dependent kinase-5. Proceedings of the National Academy of Sciences 98:12742-12747.

Li D, Specht CG, Waites CL, Butler-Munro C, Leal-Ortiz S, Foote JW, Genoux D, Garner CC, Montgomery JM (2011) SAP97 directs NMDA receptor spine targeting and synaptic plasticity. The Journal of physiology 589:4491-4510.

Liao GY, Wagner DA, Hsu MH, Leonard JP (2001) Evidence for direct protein kinase-C mediated modulation of $\mathrm{N}$-methyl-D-aspartate receptor current. Molecular pharmacology 59:960-964.

Lim ACB, Hou Z, Goh C-P, Qi RZ (2004) Protein kinase CK2 is an inhibitor of the neuronal Cdk5 kinase. The Journal of biological chemistry 279:46668-46673. 
Lisman J (1994) The CaM kinase II hypothesis for the storage of synaptic memory. Trends in neurosciences 17:406-412.

Liu GJ, Madsen BW (1997) PACAP38 modulates activity of NMDA receptors in cultured chick cortical neurons. Journal of neurophysiology 78:2231-2234.

Liu J, Chang L, Roselli F, Almeida OFX, Gao X, Wang X, Yew DT, Wu Y (2010) Amyloid- $\beta$ Induces Caspase-Dependent Loss of PSD-95 and Synaptophysin Through NMDA Receptors. Journal of Alzheimer's Disease 22:541-556.

Liu L, Wong TP, Pozza MF, Lingenhoehl K, Wang Y, Sheng M, Auberson YP, Wang YT (2004a) Role of NMDA receptor subtypes in governing the direction of hippocampal synaptic plasticity. Science (New York, NY) 304:1021-1024.

Liu X-B, Murray KD, Jones EG (2004b) Switching of NMDA receptor 2A and 2B subunits at thalamic and cortical synapses during early postnatal development. The Journal of neuroscience : the official journal of the Society for Neuroscience 24:8885-8895.

Liu X-B, Murray KD, Jones EG (2004c) Switching of NMDA Receptor 2A and 2B Subunits at Thalamic and Cortical Synapses during Early Postnatal Development. The Journal of Neuroscience 24:8885-8895.

Liu X-Y, Chu X-P, Mao L-M, Wang M, Lan H-X, Li M-H, Zhang G-C, Parelkar NK, Fibuch EE, Haines M, Neve KA, Liu F, Xiong Z-G, Wang JQ (2006) Modulation of D2R-NR2B interactions in response to cocaine. Neuron 52:897-909.

Lois C, Hong EJ, Pease S, Brown EJ, Baltimore D (2002) Germline transmission and tissuespecific expression of transgenes delivered by lentiviral vectors. Science (New York, NY) 295:868-872.

Losi G, Prybylowski K, Fu Z, Luo J, Wenthold RJ, Vicini S (2003a) PSD-95 regulates NMDA receptors in developing cerebellar granule neurons of the rat. The Journal of physiology 548:21-29.

Losi G, Prybylowski K, Fu Z, Luo J, Wenthold RJ, Vicini S (2003b) PSD-95 regulates NMDA receptors in developing cerebellar granule neurons of the rat. The Journal of Physiology 548:21-29.

Lu W, Ziff EB (2005) PICK1 interacts with ABP/GRIP to regulate AMPA receptor trafficking. Neuron 47:407-421.

Lue RA, Marfatia SM, Branton D, Chishti AH (1994) Cloning and characterization of hdlg: the human homologue of the Drosophila discs large tumor suppressor binds to protein 4.1. Proceedings of the National Academy of Sciences of the United States of America 91:9818-9822.

Lundberg KS, Shoemaker DD, Adams MW, Short JM, Sorge JA, Mathur EJ (1991) Highfidelity amplification using a thermostable DNA polymerase isolated from Pyrococcus furiosus. Gene 108:1-6. 
Lüscher C, Huber KM (2010) Group 1 mGluR-dependent synaptic long-term depression: mechanisms and implications for circuitry and disease. Neuron 65:445-459.

Macdonald DS, Weerapura M, Beazely MA, Martin L, Czerwinski W, Roder JC, Orser BA, MacDonald JF (2005) Modulation of NMDA receptors by pituitary adenylate cyclase activating peptide in CA1 neurons requires $\mathrm{G}$ alpha $\mathrm{q}$, protein kinase $\mathrm{C}$, and activation of Src. The Journal of neuroscience : the official journal of the Society for Neuroscience 25:11374-11384.

MacDonald JF, Jackson MF, Beazely MA (2007) G protein-coupled receptors control NMDARs and metaplasticity in the hippocampus. Biochimica et biophysica acta 1768:941-951.

Malenka RC, Bear MF (2004) LTP and LTD: An Embarrassment of Riches. Neuron 44:5-21.

Malenka RC, Malinow R (2011) Alzheimer's disease: Recollection of lost memories. Nature 469:44-45.

Malenka RC, Nicoll RA (1993) NMDA-receptor-dependent synaptic plasticity: multiple forms and mechanisms. Trends in neurosciences 16:521-527.

Malinow R (2003) AMPA receptor trafficking and long-term potentiation. Philosophical transactions of the Royal Society of London Series B, Biological sciences 358:707-714.

Malinow R, Malenka RC (2002) AMPA receptor trafficking and synaptic plasticity. Annual review of neuroscience 25:103-126.

Man H-Y, Sekine-Aizawa Y, Huganir RL (2007) Regulation of \{alpha\}-amino-3-hydroxy-5methyl-4-isoxazolepropionic acid receptor trafficking through PKA phosphorylation of the Glu receptor 1 subunit. Proceedings of the National Academy of Sciences of the United States of America 104:3579-3584.

Masters JR, Stacey GN (2007) Changing medium and passaging cell lines. Nature protocols 2:2276-2284.

Masuko N, Makino K, Kuwahara H, Fukunaga K, Sudo T, Araki N, Yamamoto H, Yamada Y, Miyamoto E, Saya H (1999) Interaction of NE-dlg/SAP102, a neuronal and endocrine tissue-specific membrane-associated guanylate kinase protein, with calmodulin and PSD95/SAP90. A possible regulatory role in molecular clustering at synaptic sites. The Journal of biological chemistry 274:5782-5790.

Matsuda S, Mikawa S, Hirai H (1999) Phosphorylation of serine-880 in GluR2 by protein kinase $\mathrm{C}$ prevents its $\mathrm{C}$ terminus from binding with glutamate receptor-interacting protein. Journal of neurochemistry 73:1765-1768.

Matta JA, Ashby MC, Sanz-Clemente A, Roche KW, Isaac JTR (2011) mGluR5 and NMDA receptors drive the experience- and activity-dependent NMDA receptor NR2B to NR2A subunit switch. Neuron 70:339-351. 
Mayer ML, Armstrong N (2004) Structure and function of glutamate receptor ion channels. Annual review of physiology 66:161-181.

Mayer ML, Westbrook GL, Guthrie PB (1984) Voltage-dependent block by Mg2+ of NMDA responses in spinal cord neurones. Nature 309:261-263.

McGee AW, Dakoji SR, Olsen O, Bredt DS, Lim WA, Prehoda KE (2001a) Structure of the SH3-guanylate kinase module from PSD-95 suggests a mechanism for regulated assembly of MAGUK scaffolding proteins. Molecular cell 8:1291-1301.

McGee AW, Topinka JR, Hashimoto K, Petralia RS, Kakizawa S, Kauer FW, Aguilera-Moreno A, Wenthold RJ, Kano M, Bredt DS, Kauer F (2001b) PSD-93 knock-out mice reveal that neuronal MAGUKs are not required for development or function of parallel fiber synapses in cerebellum. The Journal of neuroscience : the official journal of the Society for Neuroscience 21:3085-3091.

McMahon HT, Nicholls DG (1991) Transmitter glutamate release from isolated nerve terminals: evidence for biphasic release and triggering by localized Ca2+. Journal of neurochemistry 56:86-94.

Migaud M, Charlesworth P, Dempster M, Webster LC, Watabe AM, Makhinson M, He Y, Ramsay MF, Morris RG, Morrison JH, O’Dell TJ, Grant SG (1998) Enhanced long-term potentiation and impaired learning in mice with mutant postsynaptic density-95 protein. Nature 396:433-439.

Mohrmann R, Hatt H, Gottmann K (2000) Developmental regulation of subunit composition of extrasynaptic NMDA receptors in neocortical neurones. Neuroreport 11:1203-1208.

Montgomery JM, Zamorano PL, Garner CC (2004) MAGUKs in synapse assembly and function: an emerging view. Cellular and molecular life sciences : CMLS 61:911-929.

Monyer H, Burnashev N, Laurie DJ, Sakmann B, Seeburg PH (1994) Developmental and regional expression in the rat brain and functional properties of four NMDA receptors. Neuron 12:529-540.

Monyer H, Sprengel R, Schoepfer R, Herb A, Higuchi M, Lomeli H, Burnashev N, Sakmann B, Seeburg PH (1992) Heteromeric NMDA receptors: molecular and functional distinction of subtypes. Science (New York, NY) 256:1217-1221.

Morabito MA, Sheng M, Tsai L-H (2004) Cyclin-Dependent Kinase 5 Phosphorylates the NTerminal Domain of the Postsynaptic Density Protein PSD-95 in Neurons. J Neurosci 24:865-876.

Müller BM, Kistner U, Kindler S, Chung WJ, Kuhlendahl S, Fenster SD, Lau LF, Veh RW, Huganir RL, Gundelfinger ED, Garner CC (1996) SAP102, a novel postsynaptic protein that interacts with NMDA receptor complexes in vivo. Neuron 17:255-265. 
Naisbitt S, Kim E, Tu JC, Xiao B, Sala C, Valtschanoff J, Weinberg RJ, Worley PF, Sheng M (1999) Shank, a novel family of postsynaptic density proteins that binds to the NMDA receptor/PSD-95/GKAP complex and cortactin. Neuron 23:569-582.

Nakagawa T, Futai K, Lashuel HA, Lo I, Okamoto K, Walz T, Hayashi Y, Sheng M (2004) Quaternary structure, protein dynamics, and synaptic function of SAP97 controlled by L27 domain interactions. Neuron 44:453-467.

Nakanishi S (1992) Molecular diversity of glutamate receptors and implications for brain function. Science (New York, NY) 258:597-603.

Nakazawa T, Komai S, Tezuka T, Hisatsune C, Umemori H, Semba K, Mishina M, Manabe T, Yamamoto T (2001) Characterization of Fyn-mediated tyrosine phosphorylation sites on GluR epsilon 2 (NR2B) subunit of the N-methyl-D-aspartate receptor. The Journal of biological chemistry 276:693-699.

Nicholson DA, Trana R, Katz Y, Kath WL, Spruston N, Geinisman Y (2006) Distancedependent differences in synapse number and AMPA receptor expression in hippocampal CA1 pyramidal neurons. Neuron 50:431-442.

Niethammer M, Kim E, Sheng M (1996) Interaction between the C terminus of NMDA receptor subunits and multiple members of the PSD-95 family of membrane-associated guanylate kinases. The Journal of Neuroscience 16:2157-2163.

Niethammer M, Smith DS, Ayala R, Peng J, Ko J, Lee M-S, Morabito M, Tsai L-H (2000) NUDEL Is a Novel Cdk5 Substrate that Associates with LIS1 and Cytoplasmic Dynein. Neuron 28:697-711.

Niethammer M, Valtschanoff JG, Kapoor TM, Allison DW, Weinberg RJ, Craig AM, Sheng M (1998) CRIPT, a novel postsynaptic protein that binds to the third PDZ domain of PSD95/SAP90. Neuron 20:693-707.

Nowak L, Bregestovski P, Ascher P, Herbet A, Prochiantz A (1984) Magnesium gates glutamate-activated channels in mouse central neurones. Nature 307:462-465.

Okabe S, Kim HD, Miwa A, Kuriu T, Okado H (1999) Continual remodeling of postsynaptic density and its regulation by synaptic activity. Nature neuroscience 2:804-811.

Okabe S, Miwa A, Okado H (2001) Spine formation and correlated assembly of presynaptic and postsynaptic molecules. The Journal of neuroscience : the official journal of the Society for Neuroscience 21:6105-6114.

Paarmann I, Spangenberg O, Lavie A, Konrad M (2002) Formation of complexes between $\mathrm{Ca} 2+$.calmodulin and the synapse-associated protein SAP97 requires the SH3 domainguanylate kinase domain-connecting HOOK region. The Journal of biological chemistry 277:40832-40838.

Pak DT, Yang S, Rudolph-Correia S, Kim E, Sheng M (2001) Regulation of dendritic spine morphology by SPAR, a PSD-95-associated RapGAP. Neuron 31:289-303. 
Panula P, Sallinen V, Sundvik M, Kolehmainen J, Torkko V, Tiittula A, Moshnyakov M, Podlasz P (2006) Modulatory neurotransmitter systems and behavior: towards zebrafish models of neurodegenerative diseases. Zebrafish 3:235-247.

Paoletti P, Ascher P, Neyton J (1997) High-affinity zinc inhibition of NMDA NR1-NR2A receptors. The Journal of neuroscience : the official journal of the Society for Neuroscience 17:5711-5725.

Papouin T, Ladépêche L, Ruel J, Sacchi S, Labasque M, Hanini M, Groc L, Pollegioni L, Mothet J-P, Oliet SHR (2012) Synaptic and Extrasynaptic NMDA Receptors Are Gated by Different Endogenous Coagonists. Cell 150:633-646.

Parker MJ, Zhao S, Bredt DS, Sanes JR, Feng G (2004) PSD93 regulates synaptic stability at neuronal cholinergic synapses. The Journal of neuroscience : the official journal of the Society for Neuroscience 24:378-388.

Patrick GN, Zukerberg L, Nikolic M, De la Monte S, Dikkes P, Tsai LH (1999) Conversion of p35 to p25 deregulates Cdk5 activity and promotes neurodegeneration. Nature 402:615622.

Philpot BD, Sekhar AK, Shouval HZ, Bear MF (2001) Visual Experience and Deprivation Bidirectionally Modify the Composition and Function of NMDA Receptors in Visual Cortex. Neuron 29:157-169.

Plant K, Pelkey KA, Bortolotto ZA, Morita D, Terashima A, McBain CJ, Collingridge GL, Isaac JTR (2006) Transient incorporation of native GluR2-lacking AMPA receptors during hippocampal long-term potentiation. Nature neuroscience 9:602-604.

Polte TR, Hanks SK (1997) Complexes of Focal Adhesion Kinase (FAK) and Crk-associated Substrate (p130Cas) Are Elevated in Cytoskeleton-associated Fractions following Adhesion and Src Transformation. Journal of Biological Chemistry 272:5501-5509.

Prange O, Murphy TH (2001) Modular transport of postsynaptic density-95 clusters and association with stable spine precursors during early development of cortical neurons. The Journal of neuroscience : the official journal of the Society for Neuroscience 21:93259333.

Prybylowski K, Chang K, Sans N, Kan L, Vicini S, Wenthold RJ (2005) The synaptic localization of NR2B-containing NMDA receptors is controlled by interactions with PDZ proteins and AP-2. Neuron 47:845-857.

Raman IM, Tong G, Jahr CE (1996) Beta-adrenergic regulation of synaptic NMDA receptors by cAMP-dependent protein kinase. Neuron 16:415-421.

Raveendran R, Devi Suma Priya S, Mayadevi M, Steephan M, Santhoshkumar TR, Cheriyan J, Sanalkumar R, Pradeep KK, James J, Omkumar R V (2009) Phosphorylation status of the NR2B subunit of NMDA receptor regulates its interaction with calcium/calmodulindependent protein kinase II. Journal of neurochemistry 110:92-105. 
Rebola N, Lujan R, Cunha RA, Mulle C (2008) Adenosine A2A receptors are essential for long-term potentiation of NMDA-EPSCs at hippocampal mossy fiber synapses. Neuron 57:121-134.

Rebola N, Srikumar BN, Mulle C (2010) Activity-dependent synaptic plasticity of NMDA receptors. The Journal of physiology 588:93-99.

Reese ML, Dakoji S, Bredt DS, Dötsch V (2007) The guanylate kinase domain of the MAGUK PSD-95 binds dynamically to a conserved motif in MAP1a. Nature structural \& molecular biology 14:155-163.

Rempel-Clower NL, Zola SM, Squire LR, Amaral DG (1996) Three cases of enduring memory impairment after bilateral damage limited to the hippocampal formation. The Journal of neuroscience : the official journal of the Society for Neuroscience 16:5233-5255.

Roche KW, O'Brien RJ, Mammen AL, Bernhardt J, Huganir RL (1996) Characterization of multiple phosphorylation sites on the AMPA receptor GluR1 subunit. Neuron 16:11791188.

Roche KW, Standley S, McCallum J, Dune Ly C, Ehlers MD, Wenthold RJ (2001) Molecular determinants of NMDA receptor internalization. Nature neuroscience 4:794-802.

Rojas A, Dingledine R (2013) Ionotropic Glutamate Receptors: Regulation by G-protein Coupled Receptors. Molecular pharmacology.

Roselli F, Tirard M, Lu J, Hutzler P, Lamberti P, Livrea P, Morabito M, Almeida OFX (2005) Soluble \{beta\}-Amyloid1-40 Induces NMDA-Dependent Degradation of Postsynaptic Density-95 at Glutamatergic Synapses. J Neurosci 25:11061-11070.

Rumbaugh G, Sia G-M, Garner CC, Huganir RL (2003) Synapse-associated protein-97 isoform-specific regulation of surface AMPA receptors and synaptic function in cultured neurons. The Journal of neuroscience : the official journal of the Society for Neuroscience 23:4567-4576.

Rönicke R, Mikhaylova M, Rönicke S, Meinhardt J, Schröder UH, Fändrich M, Reiser G, Kreutz MR, Reymann KG (2011) Early neuronal dysfunction by amyloid $\beta$ oligomers depends on activation of NR2B-containing NMDA receptors. Neurobiology of aging 32:2219-2228.

Saiki RK, Gelfand DH, Stoffel S, Scharf SJ, Higuchi R, Horn GT, Mullis KB, Erlich HA (1988) Primer-directed enzymatic amplification of DNA with a thermostable DNA polymerase. Science (New York, NY) 239:487-491.

Saiki RK, Scharf S, Faloona F, Mullis KB, Horn GT, Erlich HA, Arnheim N (1985) Enzymatic amplification of beta-globin genomic sequences and restriction site analysis for diagnosis of sickle cell anemia. Science (New York, NY) 230:1350-1354.

Sambrook J (2001) Molecular Cloning: A Laboratory Manual, Third Edition (3 Volume Set). Cold Spring Harbor Laboratory Press:13. 
Sanderson TM, Collingridge GL, Fitzjohn SM (2011) Differential trafficking of AMPA receptors following activation of NMDA receptors and mGluRs. Molecular brain 4:30.

Sanhueza M, Fernandez-Villalobos G, Stein IS, Kasumova G, Zhang P, Bayer KU, Otmakhov N, Hell JW, Lisman J (2011) Role of the CaMKII/NMDA receptor complex in the maintenance of synaptic strength. The Journal of neuroscience : the official journal of the Society for Neuroscience 31:9170-9178.

Sans N, Petralia RS, Wang YX, Blahos J, Hell JW, Wenthold RJ (2000) A developmental change in NMDA receptor-associated proteins at hippocampal synapses. The Journal of neuroscience : the official journal of the Society for Neuroscience 20:1260-1271.

Sanz-Clemente A, Matta JA, Isaac JTR, Roche KW (2010) Casein kinase 2 regulates the NR2 subunit composition of synaptic NMDA receptors. Neuron 67:984-996.

Sara Y, Bal M, Adachi M, Monteggia LM, Kavalali ET (2011) Use-dependent AMPA receptor block reveals segregation of spontaneous and evoked glutamatergic neurotransmission. The Journal of neuroscience : the official journal of the Society for Neuroscience $31: 5378-5382$.

Scannevin RH, Huganir RL (2000) Postsynaptic organization and regulation of excitatory synapses. Nature reviews Neuroscience 1:133-141.

Scheiffele P, Fan J, Choih J, Fetter R, Serafini T (2000) Neuroligin expressed in nonneuronal cells triggers presynaptic development in contacting axons. Cell 101:657-669.

Schlüter OM, Schnell E, Verhage M, Tzonopoulos T, Nicoll RA, Janz R, Malenka RC, Geppert M, Südhof TC (1999) Rabphilin knock-out mice reveal that rabphilin is not required for rab3 function in regulating neurotransmitter release. The Journal of neuroscience : the official journal of the Society for Neuroscience 19:5834-5846.

Schlüter OM, Xu W, Malenka RC (2006) Alternative N-Terminal Domains of PSD-95 and SAP97 Govern Activity-Dependent Regulation of Synaptic AMPA Receptor Function. Neuron 51:99-111.

Schnell E, Sizemore M, Karimzadegan S, Chen L, Bredt DS, Nicoll RA (2002) Direct interactions between PSD-95 and stargazin control synaptic AMPA receptor number. Proceedings of the National Academy of Sciences 99:13902-13907.

Scott DB, Blanpied TA, Ehlers MD (2003) Coordinated PKA and PKC phosphorylation suppresses RXR-mediated ER retention and regulates the surface delivery of NMDA receptors. Neuropharmacology 45:755-767.

Seabold GK, Burette A, Lim IA, Weinberg RJ, Hell JW (2003) Interaction of the tyrosine kinase Pyk2 with the N-methyl-D-aspartate receptor complex via the Src homology 3 domains of PSD-95 and SAP102. The Journal of biological chemistry 278:15040-15048.

Seeburg PH (2002) A-to-I editing: new and old sites, functions and speculations. Neuron $35: 17-20$. 
Shapiro AL, Viñuela E, Maizel J V (1967) Molecular weight estimation of polypeptide chains by electrophoresis in SDS-polyacrylamide gels. Biochemical and biophysical research communications 28:815-820.

Sheng M, Cummings J, Roldan LA, Jan YN, Jan LY (1994) Changing subunit composition of heteromeric NMDA receptors during development of rat cortex. Nature 368:144-147.

Skeberdis VA, Chevaleyre V, Lau CG, Goldberg JH, Pettit DL, Suadicani SO, Lin Y, Bennett MVL, Yuste R, Castillo PE, Zukin RS (2006) Protein kinase A regulates calcium permeability of NMDA receptors. Nature neuroscience 9:501-510.

Smith WB, Starck SR, Roberts RW, Schuman EM (2005) Dopaminergic stimulation of local protein synthesis enhances surface expression of GluR1 and synaptic transmission in hippocampal neurons. Neuron 45:765-779.

Snyder EM, Nong Y, Almeida CG, Paul S, Moran T, Choi EY, Nairn AC, Salter MW, Lombroso PJ, Gouras GK, Greengard P (2005) Regulation of NMDA receptor trafficking by amyloid-beta. Nature neuroscience 8:1051-1058.

Snyder JS, Choe JS, Clifford MA, Jeurling SI, Hurley P, Brown A, Kamhi JF, Cameron HA (2009) Adult-born hippocampal neurons are more numerous, faster maturing, and more involved in behavior in rats than in mice. The Journal of neuroscience : the official journal of the Society for Neuroscience 29:14484-14495.

Sommer B, Keinänen K, Verdoorn TA, Wisden W, Burnashev N, Herb A, Köhler M, Takagi T, Sakmann B, Seeburg PH (1990) Flip and flop: a cell-specific functional switch in glutamate-operated channels of the CNS. Science (New York, NY) 249:1580-1585.

Song I, Huganir RL (2002) Regulation of AMPA receptors during synaptic plasticity. Trends in neurosciences 25:578-588.

Stein V, House DRC, Bredt DS, Nicoll RA (2003) Postsynaptic Density-95 Mimics and Occludes Hippocampal Long-Term Potentiation and Enhances Long-Term Depression. The Journal of Neuroscience 23:5503-5506.

Stelzer A, Simon G, Kovacs G, Rai R (1994) Synaptic disinhibition during maintenance of long-term potentiation in the CA1 hippocampal subfield. Proceedings of the National Academy of Sciences of the United States of America 91:3058-3062.

Stocca G, Vicini S (1998) Increased contribution of NR2A subunit to synaptic NMDA receptors in developing rat cortical neurons. The Journal of Physiology 507:13-24.

Stoppini L, Buchs PA, Muller D (1991) A simple method for organotypic cultures of nervous tissue. Journal of neuroscience methods 37:173-182.

Strack S, McNeill RB, Colbran RJ (2000) Mechanism and regulation of calcium/calmodulindependent protein kinase II targeting to the NR2B subunit of the N-methyl-D-aspartate receptor. The Journal of biological chemistry 275:23798-23806. 
Sun Q, Turrigiano GG (2011) PSD-95 and PSD-93 Play Critical But Distinct Roles in Synaptic Scaling Up and Down. The Journal of Neuroscience 31:6800-6808.

Szinyei C, Stork O, Pape H-C (2003) Contribution of NR2B subunits to synaptic transmission in amygdaloid interneurons. The Journal of neuroscience : the official journal of the Society for Neuroscience 23:2549-2556.

Takasu MA, Dalva MB, Zigmond RE, Greenberg ME (2002) Modulation of NMDA receptordependent calcium influx and gene expression through EphB receptors. Science (New York, NY) 295:491-495.

Takeuchi M, Hata Y, Hirao K, Toyoda A, Irie M, Takai Y (1997) SAPAPs. A family of PSD95/SAP90-associated proteins localized at postsynaptic density. The Journal of biological chemistry 272:11943-11951.

Terashima A, Cotton L, Dev KK, Meyer G, Zaman S, Duprat F, Henley JM, Collingridge GL, Isaac JTR (2004) Regulation of synaptic strength and AMPA receptor subunit composition by PICK1. The Journal of neuroscience : the official journal of the Society for Neuroscience 24:5381-5390.

Thomas CG, Miller AJ, Westbrook GL (2006) Synaptic and extrasynaptic NMDA receptor NR2 subunits in cultured hippocampal neurons. Journal of neurophysiology 95:17271734.

Tingley WG, Ehlers MD, Kameyama K, Doherty C, Ptak JB, Riley CT, Huganir RL (1997) Characterization of protein kinase $\mathrm{A}$ and protein kinase $\mathrm{C}$ phosphorylation of the $\mathrm{N}$ methyl-D-aspartate receptor NR1 subunit using phosphorylation site-specific antibodies. The Journal of biological chemistry 272:5157-5166.

Tomita S, Adesnik H, Sekiguchi M, Zhang W, Wada K, Howe JR, Nicoll RA, Bredt DS (2005) Stargazin modulates AMPA receptor gating and trafficking by distinct domains. Nature 435:1052-1058.

Topinka JR, Bredt DS (1998) N-Terminal Palmitoylation of PSD-95 Regulates Association with Cell Membranes and Interaction with K+ Channel Kv1.4. Neuron 20:125-134.

Tovar KR, Sprouffske K, Westbrook GL (2000) Fast NMDA receptor-mediated synaptic currents in neurons from mice lacking the epsilon2 (NR2B) subunit. Journal of neurophysiology 83:616-620.

Tovar KR, Westbrook GL (1999) The incorporation of NMDA receptors with a distinct subunit composition at nascent hippocampal synapses in vitro. The Journal of neuroscience : the official journal of the Society for Neuroscience 19:4180-4188.

Towbin H, Staehelin T, Gordon J (1979) Electrophoretic transfer of proteins from polyacrylamide gels to nitrocellulose sheets: procedure and some applications. Proceedings of the National Academy of Sciences of the United States of America $76: 4350-4354$. 
Townsend M, Yoshii A, Mishina M, Constantine-Paton M (2003) Developmental loss of miniature N-methyl-d-aspartate receptor currents in NR2A knockout mice. Proceedings of the National Academy of Sciences of the United States of America 100:1340-1345.

Tsai LH, Delalle I, Caviness VS, Chae T, Harlow E (1994) p35 is a neural-specific regulatory subunit of cyclin-dependent kinase 5. Nature 371:419-423.

Turrigiano GG (2008) The self-tuning neuron: synaptic scaling of excitatory synapses. Cell 135:422-435.

Turrigiano GG, Leslie KR, Desai NS, Rutherford LC, Nelson SB (1998) Activity-dependent scaling of quantal amplitude in neocortical neurons. Nature 391:892-896.

Valtschanoff JG, Weinberg RJ (2001) Laminar organization of the NMDA receptor complex within the postsynaptic density. The Journal of neuroscience : the official journal of the Society for Neuroscience 21:1211-1217.

Van den Haute C, Spittaels K, Van Dorpe J, Lasrado R, Vandezande K, Laenen I, Geerts H, Van Leuven F (2001) Coexpression of human cdk5 and its activator p35 with human protein tau in neurons in brain of triple transgenic mice. Neurobiology of disease 8:32-44.

Vissel B, Krupp JJ, Heinemann SF, Westbrook GL (2001) A use-dependent tyrosine dephosphorylation of NMDA receptors is independent of ion flux. Nature neuroscience 4:587-596.

Wang C-C, Held RG, Chang S-C, Yang L, Delpire E, Ghosh A, Hall BJ (2011) A critical role for GluN2B-containing NMDA receptors in cortical development and function. Neuron 72:789-805.

Washbourne P, Dityatev A, Scheiffele P, Biederer T, Weiner JA, Christopherson KS, ElHusseini A (2004) Cell adhesion molecules in synapse formation. The Journal of neuroscience : the official journal of the Society for Neuroscience 24:9244-9249.

Watkins JC (1981) Pharmacology of excitatory amino acid transmitters. Advances in biochemical psychopharmacology 29:205-212.

Watkins JC, Krogsgaard-Larsen P, Honoré T (1990) Structure-activity relationships in the development of excitatory amino acid receptor agonists and competitive antagonists. Trends in pharmacological sciences 11:25-33.

Wheeler DS, Barrick SR, Grubisha MJ, Brufsky AM, Friedman PA, Romero G (2011) Direct interaction between NHERF1 and Frizzled regulates $\beta$-catenin signaling. Oncogene $30: 32-42$.

Williams K (1993) Ifenprodil discriminates subtypes of the N-methyl-D-aspartate receptor: selectivity and mechanisms at recombinant heteromeric receptors. Molecular pharmacology 44:851-859. 
Williams K (1996) Separating dual effects of zinc at recombinant N-methyl-D-aspartate receptors. Neuroscience letters 215:9-12.

Wisden W, Seeburg PH (1993) Mammalian ionotropic glutamate receptors. Current opinion in neurobiology 3:291-298.

Wu G-M, Hou X-Y (2010) Oligomerized A[beta]25-35 induces increased tyrosine phosphorylation of NMDA receptor subunit $2 \mathrm{~A}$ in rat hippocampal CA1 subfield. Brain Research 1343:186-193.

Xu W (2011) PSD-95-like membrane associated guanylate kinases (PSD-MAGUKs) and synaptic plasticity. Current opinion in neurobiology 21:306-312.

Xu W, Schlüter OM, Steiner P, Czervionke BL, Sabatini B, Malenka RC (2008) Molecular Dissociation of the Role of PSD-95 in Regulating Synaptic Strength and LTD. Neuron 57:248-262.

Yaka R, He D-Y, Phamluong K, Ron D (2003) Pituitary adenylate cyclase-activating polypeptide (PACAP(1-38)) enhances N-methyl-D-aspartate receptor function and brainderived neurotrophic factor expression via RACK1. The Journal of biological chemistry 278:9630-9638.

Yaka R, Thornton C, Vagts AJ, Phamluong K, Bonci A, Ron D (2002) NMDA receptor function is regulated by the inhibitory scaffolding protein, RACK1. Proceedings of the National Academy of Sciences of the United States of America 99:5710-5715.

Yamazaki M, Fukaya M, Abe M, Ikeno K, Kakizaki T, Watanabe M, Sakimura K (2001) Differential palmitoylation of two mouse glutamate receptor interacting protein 1 forms with different N-terminal sequences. Neuroscience letters 304:81-84.

Yang K, Trepanier C, Sidhu B, Xie Y-F, Li H, Lei G, Salter MW, Orser BA, Nakazawa T, Yamamoto T, Jackson MF, Macdonald JF (2012) Metaplasticity gated through differential regulation of GluN2A versus GluN2B receptors by Src family kinases. The EMBO journal 31:805-816.

Yao W-D, Gainetdinov RR, Arbuckle MI, Sotnikova TD, Cyr M, Beaulieu J-M, Torres GE, Grant SGN, Caron MG (2004) Identification of PSD-95 as a regulator of dopaminemediated synaptic and behavioral plasticity. Neuron 41:625-638.

Yashiro K, Philpot BD (2008) Regulation of NMDA receptor subunit expression and its implications for LTD, LTP, and metaplasticity. Neuropharmacology 55:1081-1094.

Yoshii A, Sheng MH, Constantine-Paton M (2003) Eye opening induces a rapid dendritic localization of PSD-95 in central visual neurons. Proceedings of the National Academy of Sciences of the United States of America 100:1334-1339.

Yu XM, Askalan R, Keil GJ, Salter MW (1997) NMDA channel regulation by channelassociated protein tyrosine kinase Src. Science (New York, NY) 275:674-678. 
Zhang P, Lisman JE (2012) Activity-dependent regulation of synaptic strength by PSD-95 in CA1 neurons. Journal of Neurophysiology.

Zhang S, Edelmann L, Liu J, Crandall JE, Morabito MA (2008) Cdk5 Regulates the Phosphorylation of Tyrosine 1472 NR2B and the Surface Expression of NMDA Receptors. J Neurosci 28:415-424.

Zheng C-Y, Petralia RS, Wang Y-X, Kachar B, Wenthold RJ (2010) SAP102 is a highly mobile MAGUK in spines. The Journal of neuroscience : the official journal of the Society for Neuroscience 30:4757-4766.

Zheng C-Y, Wang Y-X, Kachar B, Petralia RS (2011) Differential localization of SAP102 and PSD-95 is revealed in hippocampal spines using super-resolution light microscopy. Communicative \& integrative biology 4:104-105.

Zheng F, Gingrich MB, Traynelis SF, Conn PJ (1998) Tyrosine kinase potentiates NMDA receptor currents by reducing tonic zinc inhibition. Nature neuroscience 1:185-191.

Ziff EB (1997) Enlightening the postsynaptic density. Neuron 19:1163-1174.

Zola-Morgan S, Squire LR, Amaral DG (1986) Human amnesia and the medial temporal region: enduring memory impairment following a bilateral lesion limited to field CA1 of the hippocampus. The Journal of neuroscience : the official journal of the Society for Neuroscience 6:2950-2967. 


\title{
Curriculum Vitae
}

\author{
Personal Data
}

Name:

S. Derya Akad

Date of birth:

$17^{\text {th }}$ November 1984

Place of birth:

BadEms, Germany

Nationality:

Turkish

Email:

deryaakad@gmail.com

\section{Education}

$04 / 2009-04 / 2013$

Doctoral studies

with Dr. Dr. Oliver M. Schlüter

European Neuroscience Institute, Göttingen, Germany

Thesis: The role of PSD-95 and kinase interactions in synaptic transmission

09/2007-04/2009

M.Sc. at IMPRS Neuroscience School with Dr. Dr. Oliver M. Schlüter

European Neuroscience Institute, Göttingen, Germany

Thesis: Phosphorylation of PSD-95 as a mechanism to regulate signaling scaffolds

$09 / 2003-06 / 2007$

B.Sc. in Molecular Biology \& Genetics

Boğaziçi University, Istanbul, Turkey 


\section{Scholarships}

$09 / 2007-09 / 2008$

International Max-Planck Research School Stipend, Germany

08/2006 DAAD International Summer School Scholarship, Germany

$09 / 2002-09 / 2003$ Boğaziçi University Success Scholarship, Turkey

Publications \& Presentations

\section{Publications}

Bonnet S.A.D ${ }^{*}$, Akad D. ${ }^{*}$, Liu Y., Dong Y.A, Schlüter O.M.

A minimal PSD-95 to autonomously enhance AMPA receptor function. (In preparation)

\section{Poster Presentations}

Akad D. and Schlüter O.M.

The role of PSD-95 and kinase interactions in synaptic transmission, FENS Meeting, Barcelona, Spain, 2012

Akad D. and Schlüter O.M.

The role of PSD-95 and kinase interactions in synaptic transmission, Meeting of the German Neuroscience Society, Göttingen, Germany, 2011

\section{Conference Talks}

Akad D. and Schlüter O.M.

The role of PSD-95 and kinase interactions in synaptic transmission, NeuroWISe Meeting, Rehovot, Israel, 2012

*: Authors contributed equally to the work 